\title{
SOCIOMATERIALITY IN CHILDREN WITH TYPICAL AND/OR ATYPICAL DEVELOPMENT
}

EDITED BY: Antonio lannaccone, Giulia Savarese, Federico Manzi and

Monica Mollo

PUBLISHED IN: Frontiers in Psychology

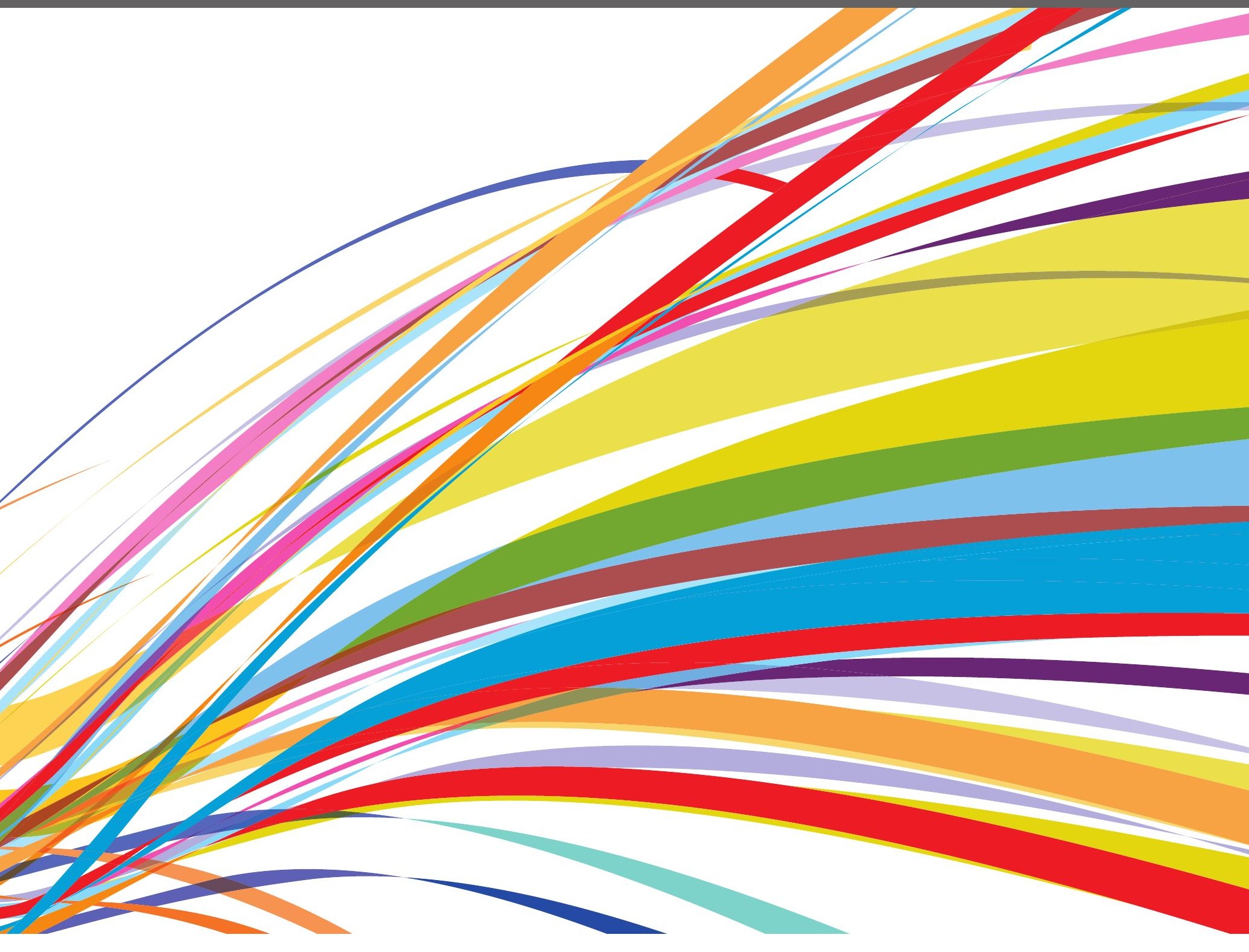

frontiers Research Topics 


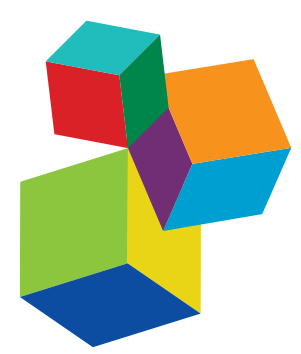

Frontiers eBook Copyright Statement

The copyright in the text of individual articles in this eBook is the property of their respective authors or their respective institutions or funders. The copyright in graphics and images within each article may be subject to copyright of other parties. In both cases this is subject to a license granted to Frontiers.

The compilation of articles constituting this eBook is the property of Frontiers.

Each article within this eBook, and the eBook itself, are published under the most recent version of the Creative Commons CC-BY licence. The version current at the date of publication of this eBook is CC-BY 4.0. If the CC-BY licence is updated, the licence granted by Frontiers is automatically updated to the new version.

When exercising any right under the CC-BY licence, Frontiers must be attributed as the original publisher of the article or eBook, as applicable.

Authors have the responsibility of ensuring that any graphics or other materials which are the property of others may be included in the

CC-BY licence, but this should be checked before relying on the

CC-BY licence to reproduce those materials. Any copyright notices relating to those materials must be complied with.

Copyright and source acknowledgement notices may not be removed and must be displayed in any copy, derivative work or partial copy which includes the elements in question.

All copyright, and all rights therein, are protected by national and international copyright laws. The above represents a summary only.

For further information please read Frontiers' Conditions for Website

Use and Copyright Statement, and the applicable CC-BY licence.

ISSN 1664-8714

ISBN 978-2-88966-434-4

DOI 10.3389/978-2-88966-434-4

\section{About Frontiers}

Frontiers is more than just an open-access publisher of scholarly articles: it is a pioneering approach to the world of academia, radically improving the way scholarly research is managed. The grand vision of Frontiers is a world where all people have an equal opportunity to seek, share and generate knowledge. Frontiers provides immediate and permanent online open access to all its publications, but this alone is not enough to realize our grand goals.

\section{Frontiers Journal Series}

The Frontiers Journal Series is a multi-tier and interdisciplinary set of open-access, online journals, promising a paradigm shift from the current review, selection and dissemination processes in academic publishing. All Frontiers journals are driven by researchers for researchers; therefore, they constitute a service to the scholarly community. At the same time, the Frontiers Journal Series operates on a revolutionary invention, the tiered publishing system, initially addressing specific communities of scholars, and gradually climbing up to broader public understanding, thus serving the interests of the lay society, too.

\section{Dedication to Quality}

Each Frontiers article is a landmark of the highest quality, thanks to genuinely collaborative interactions between authors and review editors, who include some of the world's best academicians. Research must be certified by peers before entering a stream of knowledge that may eventually reach the public - and shape society; therefore, Frontiers only applies the most rigorous and unbiased reviews.

Frontiers revolutionizes research publishing by freely delivering the most outstanding research, evaluated with no bias from both the academic and social point of view. By applying the most advanced information technologies, Frontiers is catapulting scholarly publishing into a new generation.

\section{What are Frontiers Research Topics?}

Frontiers Research Topics are very popular trademarks of the Frontiers Journals Series: they are collections of at least ten articles, all centered on a particular subject. With their unique mix of varied contributions from Original Research to Review Articles, Frontiers Research Topics unify the most influential researchers, the latest key findings and historical advances in a hot research area! Find out more on how to host your own Frontiers Research Topic or contribute to one as an author by contacting the Frontiers Editorial Office: frontiersin.org/about/contact 


\section{SOCIOMATERIALITY IN CHILDREN WITH TYPICAL AND/OR ATYPICAL DEVELOPMENT}

Topic Editors:

Antonio lannaccone, Université de Neuchâtel, Switzerland Giulia Savarese, University of Salerno, Italy Federico Manzi, Catholic University of the Sacred Heart, Milan, Italy Monica Mollo, University of Salerno, Italy

Citation: lannaccone, A., Savarese, G., Manzi, F., Mollo, M., eds. (2021). Sociomateriality in Children with Typical and/or Atypical Development. Lausanne: Frontiers Media SA. doi: 10.3389/978-2-88966-434-4 


\section{Table of Contents}

05 Editorial: Sociomateriality in Children With Typical and/or Atypical Development

Antonio lannaccone, Federico Manzi, Monica Mollo and Giulia Savarese

08 Toward a Socio-Material Approach to Cognitive Empathy in Autistic Spectrum Disorder

Antonella Marchetti, Laura Miraglia and Cinzia Di Dio

12 Transition From Crawling to Walking Changes Gaze Communication Space in Everyday Infant-Parent Interaction

Hiroki Yamamoto, Atsushi Sato and Shoji Itakura

24 The Transgenerational Consequences of the Interaction Between Humans and Molecules: Alcohol as a Cultural Artifact

Alberto Granato

29 An Old Mechanism, Imitation, Geared for Socio-Material Knowing in a "Day in the Life" of First Graders

Giuliana Pinto, Catherine Ann Cameron and Monica Toselli

36 Learning Process of Gaze Following: Computational Modeling Based on Reinforcement Learning

Mitsuhiko Ishikawa, Atsushi Senju and Shoji Itakura

46 Object Categorization Processing Differs According to Category Level: Comparing Visual Information Between the Basic and Superordinate Levels

Kosuke Taniguchi, Kana Kuraguchi, Yuji Takano and Shoji Itakura

56 Shall I Trust You? From Child-Robot Interaction to Trusting Relationships Cinzia Di Dio, Federico Manzi, Giulia Peretti, Angelo Cangelosi,

Paul L. Harris, Davide Massaro and Antonella Marchetti

70 Imitation in Chinese Preschool Children: Influence of Prior Self-Experience and Pedagogical Cues on the Imitation of Novel Acts in a Non-Western Culture

Zhidan Wang and Andrew N. Meltzoff

81 The Role of Common Ground on Object Use in Shaping the Function of Infants' Social Gaze

Nevena Dimitrova

88 On the Edge Between Digital and Physical: Materials to Enhance Creativity in Children. An Application to Atypical Development

Michela Ponticorvo, Luigia Simona Sica, Angelo Rega and Orazio Miglino

98 When the Place Matters: Moving the Classroom Into a Museum to Re-design a Public Space

Giovanna Barzanò, Francesca Amenduni, Giancarlo Cutello, Maria Lissoni, Cecilia Pecorelli, Rossana Quarta, Lorenzo Raffio, Claudia Regazzini,

Elena Zacchilli and Maria Beatrice Ligorio

105 Objects as Communicative Mediators in Children With Autism Spectrum Disorder

Federico Manzi, Giulia Savarese, Monica Mollo and Antonio lannaccone 
116 The Categorization of Objects With Uniform Texture at Superordinate and Living/Non-living Levels in Infants: An Exploratory Study

Kosuke Taniguchi, Azumi Tanabe-Ishibashi and Shoji Itakura

125 A Robot is Not Worth Another: Exploring Children's Mental State Attribution to Different Humanoid Robots

Federico Manzi, Giulia Peretti, Cinzia Di Dio, Angelo Cangelosi, Shoji Itakura,

Takayuki Kanda, Hiroshi Ishiguro, Davide Massaro and Antonella Marchetti 


\section{OPEN ACCESS}

Edited and reviewed by:

Katharina J. Rohlfing,

University of Paderborn, Germany

*Correspondence:

Federico Manzi

federico.manzi@unicatt.it

Specialty section

This article was submitted to

Developmental Psychology,

a section of the journal

Frontiers in Psychology

Received: 25 September 2020

Accepted: 20 November 2020

Published: 10 December 2020

Citation:

lannaccone A, Manzi F, Mollo $M$ and

Savarese G (2020) Editorial:

Sociomateriality in Children With Typical and/or Atypical Development.

Front. Psychol. 11:610385.

doi: 10.3389/fpsyg.2020.610385

\section{Editorial: Sociomateriality in Children With Typical and/or Atypical Development}

\author{
Antonio lannaccone ${ }^{1}$, Federico Manzi ${ }^{2 *}$, Monica Mollo ${ }^{3}$ and Giulia Savarese ${ }^{4}$ \\ ${ }^{1}$ Institut de Psychologie et Éducation, Université de Neuchâtel, Neuchâtel, Switzerland, ${ }^{2}$ Research Unit on Theory of Mind, \\ Department of Psychology, Università Cattolica del Sacro Cuore, Milan, Italy, ${ }^{3}$ Department of Human, Philosophical, and \\ Education Sciences, University of Salerno, Salerno, Italy, ${ }^{4}$ Department of Medicine, Surgery and Dentistry, University of \\ Salerno, Salerno, Italy
}

Keywords: sociomateriality, psychological development, human and non-human interactions, objects, contexts

Editorial on the Research Topic

Sociomateriality in Children With Typical and/or Atypical Development

\section{INTRODUCTION}

The idea of sociomateriality mainly originates from the vast area of perspectives on psychological development related to empiricism. In simple terms, it could be said that sociomateriality stresses the contribution of individual and collective experience by putting more emphasis on the role that corporeity, physical contexts, and objects play in the development or emergence of psychological functions. Unfortunately, like any simplification, this one has objective limits. What makes it difficult to establish a unified framework to define sociomateriality, and above all to determine its relationship to psychological development, is first of all an epistemological question that is still the subject of a wide debate in several scientific areas, including philosophy (Searle, 2007) archaeology and material cultures (Malafouris, 2013), ergonomics (Geslin, 2017), anthropology and sociology (Latour, 2005), cognitive sciences (Clark, 2008), psychotherapy (Searles, 1960), developmental psychology (Moro and Rodríguez, 1998; Moro, 2016; Iannaccone et al., 2018) and learning itself (Engeström, 2015; Iannaccone, 2017; Cattaruzza et al., 2019). Within the limited extent of this introduction to the variegated Topic hosted by Frontiers in Psychology, we can identify the heart of the epistemological problem in two fundamental questions: (a) what are the boundaries of the mind with respect to corporeity and the context in which it operates? and (b) what could be the real contribution that artifacts give to the development of psychological functions, particularly learning?

Of course, these two problems not only have an abstract philosophical meaning, but also constitute a real methodological puzzle, because they question the notions of "object of analysis" and "unity of analysis." To these important problems, researchers have given varied answers that are arranged along an axis with what we could define as "strong sociomateriality" on the one end and "weak sociomateriality" on the other. Concerning the explanations of psychological phenomena, this continuum depends substantially on the more or less decisive role that researchers assign to both the physical characteristics (materialities) of objects or contexts and to the communicative and semiotic interactions between humans and non-humans (social and cultural mediations). Even within this Topic, which is specifically dedicated to the role of objects in psychological development (affective, cognitive, and social), the contributions collected do not refer to a single notion of 
sociomateriality. On the positive side, these contributions present a rich landscape of theoretical and empirical positions requiring the reader to seriously reconsider sociomateriality in psychology. In summarizing the 14 contributions, we identified some common general aspects of the Topic that can help the reader organize his or her "journey": Mental activities are not considered as decontextualized and isolated, but are interwoven in the interactions among individuals on one hand and the physical and social worlds on the other; and objects seem to actively contribute to typical and atypical psychological development (cognitive, affective, and social), influencing to several degrees the way that people experience the world. The contributions to the Topic are briefly presented below, organized according to their contribution to the issue of sociomateriality in psychology.

\section{SPACES OF ACTIVITY AND OBJECTS}

The function of the physical "spaces of activity" in children's learning clearly emerges in the case report of Barzanò et al. As in other similar research, the authors support the idea of "extended and situated" learning. This perspective integrates the formal context of the school with the informal spaces of other micro-contexts in which children and adults have their daily life experiences. Alternative spaces of activity influence the complexities of relationships and thus offer new opportunities to acquire alternative ways of exploring reality and learning.

Pinto et al. adopt sociomateriality as a theoretical lens to investigate how imitation acts to support the acquisition of the use of objects. Imitation, in the opinion of the authors, is a complex activity, involving several actors who interact to facilitate the understanding of various artifacts in different domains of knowledge and improve their interpretative flexibility between communities of practice.

Even at a much earlier stage of development, the importance of objects (among other variables) as elements of the newborn/parents' space of activity is partly highlighted in the research of Yamamoto et al. For example, the number of objects on the floor between infants and parents seems to modulate the eye contact with parents and activate some changes in the way that the child explores the surrounding context.

Granato analyses the neuropsychological effects of alcohol abuse, considering it as a real artifact both in its individual "use" as well as from an intergenerational perspective. Alcohol as a cultural artifact takes on different meanings depending on the individual and social practices that characterize its excessive intake.

Wang and Meltzoff describe a study with Chinese preschool children. They report a number of theoretical views on imitation, assuming that it plays an important role in the early socio-cognitive development of children. One of the theoretical points of view refers to imitation as an act of social affiliation between the child and the adult, an activity also linked to sociomateriality. Through a series of tests of imitation with objects, researchers identified imitative activity as a key mechanism in the acquisition of culturally appropriate behaviors and conventions.

\section{OBJECTS AS MEDIATORS OF SOME COMPLEX PSYCHOLOGICAL FUNCTIONS IN CHILDREN WITH AUTISM SPECTRUM DISORDERS (ASD)}

Manzi et al. adopt a sociomaterial approach to analyse interactions among children with ASD, adults and objects in a play setting. Systematic observations of object manipulation and communicative patterns displayed by children are conducted.

Assuming a perspective inspired by the Vygotskian concept of "psychological tools," Manzi et al. highlight how objects can be considered (in some cases) as helpful mediators of communication, even in situations where interactions seem very problematic. The work also suggests a promising approach to supporting communicative patterns in children with ASD.

Marchetti et al. argue that motivated and shared actions directed toward an object can effectively mediate the child-adult relationship. According to this work, when face-to-face communication is challenging, as it is for example children with ASD, the presence of an object that encourages playful activities can expand the possibilities of communication by creating a triadic (child-adult) relationship.

Ponticorvo et al. explore the interweaving of cognitive and emotional dimensions with the nature of the materials used during the creative activities of children with ASD. The article shows how educational materials presented in both digital and physical form can effectively stimulate creativity.

Dimitrova's theoretical paper shows the importance of common background in communication between children and their parents in early childhood. Especially when the communicative setting refers to conventional uses of objects, common ground is an essential condition for a proper interpretation of the situation. In particular, a common background allows parents a well-adapted tailoring of their communicative response to the infant's developmental need.

\section{INTERACTION WITH COMPLEX OBJECTS: HUMAN-ROBOT INTERACTIONS}

The research of $\mathrm{Di}$ Dio et al. investigates the dynamics of trust (acquisition, loss and restoration) in children who interact with a humanoid robot or a human. The results show how in certain conditions, material artifacts can become referents with which to build relationships, modulated by the degree of anthropomorphization of the robotic agent.

The research of Manzi et al. shows how the degree of anthropomorphization affects the attribution of mental abilities to a robot by children of different ages. The results show that older children are more sensitive to the material characteristics of robots than younger children.

\section{PERCEPTION AND CATEGORIZATION OF OBJECTS}

The study by Ishikawa et al. simulated the learning process of the child's gaze following: it emerged that the most feasible model 
is one in which communication signals influence the child's internal states. The model presented by the authors highlighted the importance of objects as a variable to be analyzed with respect to the communicative value of the adult's gaze.

The study of Taniguchi et al. analyses the way that infants classify objects at superordinate levels, considering the categories "living" or "not living." The study shows how the categorization of living objects depends on linguistic development. According to the authors, this suggests different mechanisms in infants' categorization of living and non-living objects.

In further research, Taniguchi et al. investigate whether infants' decision to categorize objects depends on bottomup and/or top-down processing (in relation to visual or verbal presentations). The authors also try to determine

\section{REFERENCES}

Cattaruzza, E., Ligorio, M. B., and Iannaccone, A. (2019). Sociomateriality as a partner in the polyphony of students positioning. Learn. Cult. Soc. Interact. 22:100332. doi: 10.1016/j.lcsi.2019.100332

Clark, A. (2008). Supersizing the Mind. Oxford: Oxford University Press.

Engeström, Y. (2015). Learning by Expanding: An Activity-Theoretical Approach to Developmental Research, 2nd Edn. Oxford: Cambridge University Press.

Geslin, P. (2017). Inside Anthropotechnology: User and Culture Centered Experience. New York, NY: John Wiley and Sons.

Iannaccone, A. (2017). "Eduquer peut-être dur! Quelques notes autour de la notion de matérialité en éducation," in Les interactions sociales en classe: réflexions et perspectives, eds F. Arcidiacono and M. Giglio (Berne: Peter Lang), 97-122.

Iannaccone, A., Savarese, G., and Manzi, F. (2018). Object use in children with autism: building with blocks from a piagetian perspective. Front. Educ. 3:12. doi: $10.3389 /$ feduc. 2018.00012

Latour, B. (2005). Reassembling the Social: An Introduction to Actor-NetworkTheory. Oxford: Oxford University Press.

Malafouris, L. (2013). How Things Shape the Mind: A Theory of Material Engagement. Cambridge, MA: MIT Press.

Moro, C. (2016). To encounter, to build the world and to become a human being. Advocating for a material-cultural turn in developmental psychology. Integr. Psychol. Behav. Sci. 50, 586-602. doi: 10.1007/s12124-016-9356-4 what visual information is required for quick and accurate categorization.

\section{AUTHOR CONTRIBUTIONS}

All authors listed have made a substantial, direct and intellectual contribution to the work, and approved it for publication.

\section{ACKNOWLEDGMENTS}

Guest editors would like to thank all the authors and reviewers who agreed to participate in this special issue with their original contributions.

Moro, C., and Rodríguez, C. (1998). “Towards a pragmatical conception of the object: the construction of the uses of the objects by the baby in the prelinguistic period," in Child Development Within Culturally Structured Environments: Construction of Psychological Processes in Interpersonal Communication, Vol. 4, eds M. C. D. P. Lyra and J. Valsiner (Stamford, CT: Ablex Publishing Corporation), 53-72.

Searle, J. R. (2007). "Social ontology and the philosophy of society", in Creation of the Mind, eds E. Margolis and S. Laurence (Oxford: Oxford University Press), 3-17.

Searles, H. F. (1960). The Nonhuman Environment; in Normal Development and in Schizophrenia. Madison, CT: International Universities Press.

Conflict of Interest: The authors declare that the research was conducted in the absence of any commercial or financial relationships that could be construed as a potential conflict of interest.

Copyright (C) 2020 Iannaccone, Manzi, Mollo and Savarese. This is an open-access article distributed under the terms of the Creative Commons Attribution License (CC $B Y)$. The use, distribution or reproduction in other forums is permitted, provided the original author(s) and the copyright owner(s) are credited and that the original publication in this journal is cited, in accordance with accepted academic practice. No use, distribution or reproduction is permitted which does not comply with these terms. 


\section{OPEN ACCESS}

Edited by:

Giulia Savarese

University of Salerno, Italy

Reviewed by:

Rosa Angela Fabio,

University of Messina, Italy

Santo Di Nuovo,

University of Catania, Italy

*Correspondence:

Cinzia Di Dio

Cinzia.didio@unicatt.it

Specialty section: This article was submitted to

Developmental Psychology, a section of the journal

Frontiers in Psychology

Received: 02 December 2019 Accepted: 16 December 2019

Published: 10 January 2020

Citation:

Marchetti A, Miraglia L and Di Dio C (2020) Toward a Socio-Material Approach to Cognitive Empathy in

Autistic Spectrum Disorder.

Front. Psychol. 10:2965.

doi: 10.3389/fpsyg.2019.02965

\section{Toward a Socio-Material Approach to Cognitive Empathy in Autistic Spectrum Disorder}

\author{
Antonella Marchetti ${ }^{1,2}$, Laura Miraglia ${ }^{2}$ and Cinzia Di Dio ${ }^{1,2 *}$ \\ ${ }^{1}$ Research Unit on Theory of Mind, Department of Psychology, Università Cattolica del Sacro Cuore, Milan, Italy, ${ }^{2}$ Faculty of \\ Education, Università Cattolica del Sacro Cuore, Milan, Italy
}

Keywords: Theory of Mind, cognitive empathy, ToM precursors, ASD, child-adult relationship

In everyday life, actions and decisions are defined by a continuous interplay between cognitive and affective dimensions (Milani and Gagliardi, 2013; Lombardi et al., 2017) where empathy plays a central role (Eisenberg and Strayer, 1987). Empathy underpins the synchronous social response to another person's mental state and behavior. It represents a particular experience because it is not a directly accessible state: in contrast to other psychological constructs, it is neither a conduct, nor is always evident through specific behavioral expressions (Bonino et al., 1998). Empathy is like a dance between two individuals whose steps move between cognition and affects. Within a socio-material approach to development, in this paper we argue that motivated shared goaldirected actions toward an object may effectively mediate child-adult relationship by acting on the precursors of Theory of Mind (ToM), a cognitive component of empathy.

Feshbach (1978) defined empathy the philosopher's stone of human relationships. In this sense, it represents one of the most important mechanisms that contributes to regulate social relationships and human communication. Its two-dimensional nature-affective and cognitive-has spurred the interest of many authors who have tried to describe its evolution and development. According to Hoffman (1984), and in line with Davis et al. (1994) account, empathy manifests itself from the first days of life, initially quite entirely on the affective level. During the first year of life, children display motor mimicry by answering to the emotions they are witnessing (e.g., by crying when another infant is crying). This process is plausibly mediated by activation of the mirror mechanism allowing motor simulation (for review, see Rizzolatti and Sinigaglia, 2008) and "altercentric" participation (Braten, 1998). In this phase, the emotional response is involuntary and undifferentiated (Global empathy, Hoffman, 1984). Going through egocentric empathy during the second year of life, children spontaneously offer a kind of help which they would find comforting themselves and, in this sense, empathy is egocentric, although forms of early reasoning about the other's desires was observed already in 18-month-olds (Repacholi and Gopnik, 1997; see also, Astington et al., 1988). Empathy for another's feelings at the age of 3 years involves the development of role-taking skills, and children become aware that other people's feelings can differ from their own. The development of Theory of Mind (ToM), and namely the ability to conceptualize one's own and others' mental states underlying behavior (Wimmer and Perner, 1983) and social competence (Premack and Woodruff, 1978; Wellman et al., 2001), marks this maturation period. Children's responses to distress might become more appropriate to the other person's needs. We can now speak of empathy in its full meaning indicating that the child has developed the cognitive prerequisites enabling her/him to understand the other as a distinct person from her/himself (Bonino et al., 1998).

\section{EMPATHY IN ATYPICAL DEVELOPMENT}

Brain-imaging studies suggest that different but interacting brain structures are involved in cognitive and affective empathy (Shamay-Tsoory et al., 2009; Kalbe et al., 2010). These studies 
hypothesize that each individual component of the construct "empathy" can be selectively compromised with consequent specificities in behavioral impairments.

The Autistic Spectrum Disorder (ASD) is featured by two main types of impairment affecting social and language competencies, on the one hand, and involving stereotypical and repetitive behaviors, on the other (American Psychiatric Association, 2013, APA). One of the central issues is if and how ASD individuals empathize. Eye tracking experiments (Frith, 2003) have shown that autistic individuals, when looking for the meaning of a dynamic social situation, do not direct attention to very expressive aspects of the image. For example, they attend more to the peripheral area of the face instead of the eyes and mouth to which particular attention is typically paid (e.g., Savazzi et al., 2014; Di Dio et al., 2019). Furthermore, ASD individuals' mind-blindness (Baron-Cohen, 1995) leads to the inability to attribute mental states and to an inadequate conception of feelings (Baron-Cohen et al., 2001; Frith, 2003; Fabio et al., 2011). ASD individuals are generally unable to recognize and name emotions, read expressive cues and take the others' point of view and perspective, reasons that may-at least partly-explain ASD general inability to provide appropriate emotional responses. Not only mentalization skills allow people to understand the other's emotions, but importantly the reasons underlying them, thus making it is possible to respond appropriately to the other person's state of mind. If we see a sad person, it is thanks to our mentalization skills that we can understand if s/he needs relief or prefers staying alone. Nevertheless, ASD individuals are not indifferent to the other's suffering. They are able to instinctive sympathy (Frith, 2003), i.e., to involuntary respond to basic emotional reactions, although their inability to readily and coherently attribute a mental state will most likely lead to a socio-behavioral failure, with possible consequent experience of depression and anxiety (Conti et al., 2015).

\section{CAN A SOCIO-MATERIAL APPROACH PROMOTE EMPATHY?}

Socio-materiality is clearly the fusion between the terms "social" and "material," and it has the potential to link materiality to each and every phenomenon that we consider social (Leonardi et al., 2012). The socio-material theory assumes that human activity is mediated by tools (Leonardi, 2012). Actions supported by tool-use and intentionally aimed at production create thought (Vygotsky, 1978).

The conceptual underlie of our argumentation resides in the acknowledged nature of the dyadic relationship that commonly develops between the child and the adult in early childhood. In typical development, the dyadic relationship generally invites the triadic relationship by including the use of an object in its (culturally determined) typical function (Leontiev, 1981; Costall, 1997; Rodríguez and Moro, 2008; Barthélémy-Musso et al., 2013). Adults and children readily construct action representations organized with respect to an ultimate goal, allowing one to predict the consequences of action, interpret and describe actions, and categorize action sequences (Sommerville et al., 2005). Already 1-year-old infants possess a genuine understanding of other persons as intentional and attentional agents (Tomasello and Haberl, 2003). In a compromised dyadic relationship, where the infant fails or struggles to include the other in her/his action zone and mental sphere, the object may become the primary referent of the dyadic relationship. The child concentrates on the relationship with the object most possibly because s/he may ignore the emotional and mental pressures that characterize the typical relationship with the adult (Lecciso et al., 2013). The socio-material perspective suggests that through the initiation of a dyadic relationship with the object, it is possible to include the adult in the triadic relationship. So, from a typical child-adult-object interaction, the relationship shifts to the child-object-adult interaction. That objects can become the mediators of a compromised adult-child social interactions associated with attentional deficits has been already theorized (Rodríguez and Moro, 2008; Sinha and Rodríguez, 2008; Sinha, 2015). The human psychological structure is modeled and transformed by acting in the world and manipulating objects. Gradually, as the physical/sensori-motor representation of the world-which also includes the relation with the adult (see, Braten, 2006) - the child builds a representation of the mental self and the mental other. This development is not abrupt, but moves from a concrete stage to a representational stage also thanks to the developmental of processes and behaviors recognized as the precursors of ToM.

Before the acquisition of false belief at about 4 years of age, the child progressively builds an understanding of the mind. In typical development, the dyadic relationship (classically affective in nature) with the caregiver opens the child up to a triadic relationship with the world through the precursors of ToM that develop within the first 2 years of life: jointattention, pointing (indicative), performative (from requesting to declarative), understanding of agency, pretend play. In a compromised child-adult relationship, object may possibly bring the child closer to the other during shared goal-directed actions on an object. Under this condition, the child-adult's responses are contingent on a common object of interest (motivation and openness). When the other responds to the stimulus in the same way as does the child, an initial "like me" relational form may develop which starts from the child's experience of the other's objective/concrete and sensori-motor characteristics. Subsequently, the child may begin to form an association between the self and the other that includes subjective characteristics (both subjects are the same and different from the object) that are discovered through doing. Then, by intervening on the precursors of ToM, and in particular on joint attention, referential communication, and motivation, the other (and her/his mind) may be gradually included as a referential agent in a triadic relationship. A differentiation is initiated which potentially leads the child to the understanding of the other as an individual with a mind that may be different from her/his own.

The observational work by Iannaccone et al. (2018) right supports this idea. The authors preliminarily showed that objects may serve as concrete mediators in the intersubjective space between adult and ASD infants aged between 18 and 24 months during object manipulation and building a tower of toy blocks. 
Additionally, some of the infants' attention patterns were visibly mediated by the object, in that the children monitored the adult's attention through eye contact or by restarting manipulation of the blocks, a process labeled "object-mediated attention." In clinical settings, the practice of including objects during therapy is already widely used as a means to establishing a connection with the patient. In this respect, Conti et al. (2015) suggest thatby generating a high degree of motivation and engagement in the child (Scassellati, 2002) - the use of robots can be effectively integrated in current ASD therapies by developing protocols aimed to implement, for example, imitation skills, which are basic to the development of social competencies. The object "robot" becomes a mediator of the child-adult relationship. In principal, by working on ToM precursors through mediation of object-use, mind understanding and social-communicative competencies may be promoted in ASD individuals with and without intellectual disability: what matters is, in fact, the scaffolder's ability to properly place her/himself within the child's zone of proximal development to enhance her/his abilities at any level of intellectual functioning (see, Conti et al., 2018; see also, Fabio et al., 2013).

Concluding, the development of the grasp of the other's mind in terms of emotions, intentions, desires and-then-beliefs and false beliefs at increasing levels of cognitive complexity is important because, with it, the child begins to reason in terms of subjectively founded "true and false" and no longer in terms of objective "true and false." To understand that the mind represents the world and that mental representations guide action is the crucial step for the acquisition of ToM. Theory of Mind involves a representation of the subjectivity of one's own and the other's mental states at a high degree of interindividual variability. When the dyadic component is

\section{REFERENCES}

American Psychiatric Association (2013). Diagnostic and Statistical Manual of Mental Disorders (DSM- $\left.{ }^{\circledR}\right)$. Washington, DC: American Psychiatric Pub.

Astington, J. W., Harris, P. L., and Olson, D. R. (1988). Developing Theories of Mind. Cambridge, England: Cambridge University Press.

Baron-Cohen, S. (1995). Mindblindness. Cambridge, MA: MIT Press.

Baron-Cohen, S., Wheelwright, S., Hill, J., Raste, Y., and Plumb, I. (2001). The "Reading the Mind in the Eyes" Test revised version: a study with normal adults, and adults with Asperger syndrome or high-functioning autism. J. Child Psychol. Psychiatry Allied Discipl. 42, 241-251. doi: 10.1111/1469-7610.00715

Barthélémy-Musso, A., Tartas, V., and Guidetti, M. (2013). Prendre les objets et leurs usages au sérieux: approche développementale de la coconstruction de conventions sémiotiques entre enfants. Psychol. Française 58, 67-88. doi: 10.1016/j.psfr.2012.10.001

Begeer, S., Gevers, C., Clifford, P., Verhoeve, M., Kat, K., Hoddenbach, E., et al. (2011). Theory of mind training in children with autism: a randomized controlled trial. J. Autism Dev. Disord. 41, 997-1006. doi: 10.1007/s10803-010-1121-9

Bonino, S., Coco, A. L., and Tani, F. (1998). Empatia: i Processi di Condivisione delle Emozioni. Firenze: Giunti Editore.

Braten, S. (1998). "Infant learning by altercentric participation: the reverse of egocentric observation in autism," in Intersubjective Communication and Emotion in Early Ontogeny, ed S. Braten (Cambridge: Cambridge University Press), 105-124. impaired, by having the child establishing a relationship with the material object, the object can, from a socio-material perspective, open the child up to a triadic relationship (childobject-adult). This, in turn, may help the "motivated" child enter the dyadic child-adult relationship in a backward path, thus allowing the recovery and implementation of-at least someprecursors of the ToM competence. The meaning of the objects helps the individual build a meaning of the person (mind). Interventions have been developed worldwide to improve ToM skills of individuals with autism. Despite these efforts, little is known about whether, when, where and for whom these treatment programs work in autism (Begeer et al., 2011). We believe that it could be helpful to look into methods of intervention which embrace a socio-material perspective allowing to promote empathic skills by starting from its basic relational components. Our suggestion to intervene timely on ToM precursors is in line with general emphasis on early diagnosis. According to Bruner (1986), before children have acquired the "certificate" of false belief, adults and children had made a very long journey toward each other's mind. And, surely, it is in Bruner's vigoskijan spirit that this journey is populated with objects.

\section{AUTHOR CONTRIBUTIONS}

All authors listed have made a substantial, direct and intellectual contribution to the work, and approved it for publication.

\section{FUNDING}

This work was funded by Universitã Cattolica del Sacro Cuore, Milan.
Braten, S. (2006). Intersubjective Communication and Emotion in Early Ontogeny. Cambridge, UK: Cambridge University Press.

Bruner, J. (1986). Actual Minds, Possible Worlds. Cambridge, MA: Harvard University Press.

Conti, D., Di Nuovo, A., Trubia, G., Buono, S., and Di Nuovo, S. (2018). "Adapting robot-assisted therapy of children with autism and different levels of intellectual disability: a preliminary study," in 13th Annual ACM/IEEE International Conference on Human Robot Interaction, HRI (Chicago, IL), 91-92.

Conti, D., Di Nuovo, S., Buono, S., Trubia, G., and Di Nuovo, A. (2015). "Use of robotics to stimulate imitation in children with Autism Spectrum Disorder: a pilot study in a clinical setting," in RO-MAN 24th IEEE International Symposium on Robot and Human Interactive Communication (Kobe), 1-6. doi: 10.1109/ROMAN.2015.7333589

Costall, A. P. (1997). The meaning of things. Soc. Anal. 41, 76-86.

Davis, M. H., Luce, C., and Kraus, S. J. (1994). The heritability of characteristics associated with dispositional empathy. J. Pers. 62, 369-391. doi: 10.1111/j.1467-6494.1994.tb00302.x

Di Dio, C., Massaro, D., Savazzi, F. A., Gallese, V., Garau, T., Gilli, G., et al. (2019). Beauty in life: an eye-tracking study on young adults' aesthetic evaluation and vitality judgment of pictorial representations of sleeping and dead subjects. PsyCh J. doi: 10.1002/pchj.285. [Epub ahead of print].

Eisenberg, N., and Strayer, J. (1987). "Critical issues in the study of empathy," in Cambridge Studies in Social and Emotional Development. Empathy and its Development, eds N. Eisenberg and J. Strayer (New York, NY: Cambridge University Press), 3-13. 
Fabio, R. A., Castelli, I., Marchetti, A., and Antonietti, A. (2013). Training communication abilities in Rett Syndrome through reading and writing. Front. Psychol. 4:911. doi: 10.3389/fpsyg.2013.00911

Fabio, R. A., Oliva, P., and Murdaca, A. M. (2011). Systematic and emotional contents in overselectivity processes in autism. Res. Autism Spectr. Disord. 5, 575-583. doi: 10.1016/j.rasd.2010.07.001

Feshbach, N. (1978). Studies of empathic behavior in children. Prog. Exp. Pers. Res. $8,1-47$.

Frith, U. (2003). Autism: Explaining the Enigma. Oxford: Blackwell Publishing.

Hoffman, M. L. (1984). "Interaction of affect and cognition in empathy," in Emotions, Cognition, and Behavior, eds C. Izard, J. Kagan, and R. Zajonc (New York, NY: Cambridge University Press), 103-131.

Iannaccone, A., Savarese, G., and Manzi, F. (2018). Object use in children with autism: building with blocks from a Piagetian perspective. Front. Educ. 3:12. doi: 10.3389/feduc.2018.00012

Kalbe, E., Schlegel, M., Sack, A. T., Nowak, D. A., Dafotakis, M., Bangard, C., et al. (2010). Dissociating cognitive from affective theory of mind: a TMS study. Cortex 46, 769-780. doi: 10.1016/j.cortex.2009.07.010

Lecciso, F., Petrocchi, S., Savazzi, F., Marchetti, A., Nobile, M., and Molteni, M. (2013). The association between maternal resolution of the diagnosis of autism, maternal mental representations of the relationship with the child, and children's attachment. Life Span Disabil. 16, 21-38.

Leonardi, P. M. (2012). "Materiality, sociomateriality, and socio-technical systems: What do these terms mean? How are they different? Do we need them?" in Materiality and Organizing: Social Interaction in a Technological World, eds P. M. Leonardi, A. B. Nardi, and J. Kallinikos (Oxford: Oxford University Press), 25-48.

Leonardi, P. M., Nardi, B. A., and Kallinikos, J. (2012). Materiality and Organizing: Social Interaction in a Technological World. Oxford: Oxford University Press.

Leontiev, A. N. (1981). Problems of the Development of Mind. Moscow: Progress Publishers.

Lombardi, E., Di Dio, C., Castelli, I., Massaro, D., and Marchetti, A. (2017). Prospective thinking and decision making in primary school age children. Heliyon 3:e00323. doi: 10.1016/j.heliyon.2017.e00323

Milani, L., and Gagliardi, G. (2013). Fattori di rischio e di protezione nella valutazione delle competenze parentali di famiglie italiane e famiglie immigrate. Maltrattamento e Abuso all'Infanzia 15, 59-80. doi: 10.3280/MAL2013-001004

Premack, D., and Woodruff, G. (1978). Does the chimpanzee have a theory of mind? Behav. Brain Sci. 1, 515-526. doi: 10.1017/ S0140525X00076512

Repacholi, B. M., and Gopnik, A. (1997). Early reasoning about desires: evidence from 14-and 18-month-olds. Dev. Psychol. 33, 12-21. doi: $10.1037 / / 0012-1649.33 .1 .12$

Rizzolatti, G., and Sinigaglia, C. (2008). Mirrors in the Brain: How Our Minds Share Actions and Emotions. Oxford: Oxford University Press.

Rodríguez, C., and Moro, C. (2008). "Coming to agreement: object use by infants and adults," in The Shared Mind: Perspectives on Intersubjectivity, eds J. Zlatev,
T. P. Racine, C. Sinha, and E. Itkonen (Amsterdam: John Benjamins Publishing Company), 89-114.

Savazzi, F., Massaro, D., Di Dio, C., Gallese, V., Gilli, G., and Marchetti, A. (2014). Exploring responses to art in adolescence: a behavioral and eye-tracking study. PLoS ONE 9:e102888. doi: 10.1371/journal.pone.0102888

Scassellati, B. (2002). Theory of mind for a humanoid robot. Auton. Robots 12, 13-24. doi: 10.1023/A:1013298507114

Shamay-Tsoory, S. G., Aharon-Peretz, J., and Perry, D. (2009). Two systems for empathy: a double dissociation between emotional and cognitive empathy in inferior frontal gyrus versus ventromedial prefrontal lesions. Brain 132, 617-627. doi: 10.1093/brain/awn279

Sinha, C. (2015). Language and other artifacts: socio-cultural dynamics of niche construction. Front. Psychol. 6:1601. doi: 10.3389/fpsyg.2015. 01601

Sinha, C., and Rodríguez, C. (2008). "Language and the signifying object. From convention to imagination," in The Shared Mind. Perspectives on Intersubjectivity, eds J. Zlatev, T. Racine, Ch. Sinha, and E. Itkonen (Amsterdam: John Benjamins Publishing Company), 357-378.

Sommerville, J. A., Woodward, A. L., and Needham, A. (2005). Action experience alters 3-month-old infants' perception of others' actions. Cognition 96, B1-B11. doi: 10.1016/j.cognition.2004 07.004

Tomasello, M., and Haberl, K. (2003). Understanding attention: 12-and 18month-olds know what is new for other persons. Dev. Psychol. 39, 906. doi: 10.1037/0012-1649.39.5.906

Vygotsky, L. S. (1978). Socio-Cultural Theory. Mind in Society: The Development of Higher Psychological Processes. Cambridge: Harvard University Press.

Wellman, H. M., Cross, D., and Watson, J. (2001). Metaanalysis of theory-of-mind development: the truth about false belief. Child Dev. 72, 655-684. doi: 10.1111/1467-8624 00304

Wimmer, H., and Perner, J. (1983). Beliefs about beliefs: representation and constraining function of wrong beliefs in young children's understanding of deception. Cognition $13,103-128$.

Conflict of Interest: The authors declare that the research was conducted in the absence of any commercial or financial relationships that could be construed as a potential conflict of interest.

Copyright (c) 2020 Marchetti, Miraglia and Di Dio. This is an open-access article distributed under the terms of the Creative Commons Attribution License (CC BY). The use, distribution or reproduction in other forums is permitted, provided the original author(s) and the copyright owner(s) are credited and that the original publication in this journal is cited, in accordance with accepted academic practice. No use, distribution or reproduction is permitted which does not comply with these terms. 


\section{OPEN ACCESS}

Edited by: Giulia Savarese,

University of Salerno, Italy

Reviewed by:

John Franchak,

University of California, Riverside,

United States

David lan Anderson,

San Francisco State University,

United States

*Correspondence:

Hiroki Yamamoto

yamamoto.hiroki.nr@gmail.com

Specialty section:

This article was submitted to

Developmental Psychology,

a section of the journal

Frontiers in Psychology

Received: 04 October 2019 Accepted: 17 December 2019

Published: 24 January 2020

Citation:

Yamamoto $H$, Sato $A$ and Itakura $S$ (2020) Transition From Crawling to

Walking Changes Gaze Communication Space in Everyday

Infant-Parent Interaction.

Front. Psychol. 10:2987.

doi: 10.3389/fpsyg.2019.02987

\section{Transition From Crawling to Walking Changes Gaze Communication Space in Everyday Infant-Parent Interaction}

\author{
Hiroki Yamamoto ${ }^{1 *}$, Atsushi Sato ${ }^{2}$ and Shoji Itakura ${ }^{1,3}$ \\ ${ }^{1}$ Graduate School of Letters, Kyoto University, Kyoto, Japan, ${ }^{2}$ Faculty of Human Development, University of Toyama, \\ Toyama, Japan, ${ }^{3}$ Center for Baby Science, Doshisha University, Kizugawa, Japan
}

Acquisition of walking changes not only infants' locomotion itself but also infants' exploratory behavior and social interaction, such as gaze communication. To understand the ecological context in which gaze communication occurs and how it changes with walking development from the point of view of the spatial arrangement of infants, parents, and objects, we analyzed longitudinal data of daily eye contact scenes recorded from head-mounted eye trackers worn by parents as infants grew from 10 to 15.5 months, focusing on infant-parent distance and the number of objects between the dyad. A Bayesian state-space model revealed that the interpersonal distance at which infants initiated eye contact with their parents increased with the time ratio of walking to crawling. This result could not be explained by the developmental change in the amount of time that the infants were far from the parents, which is not limited to the gaze communication context. Moreover, the interpersonal distance at which the parents initiated eye contact with the infants did not increase with the time ratio of walking to crawling. The number of objects on the floor between infants and parents at the time of eye contact increased with interpersonal distance. Taken together, these results indicate that the transition from crawling to walking changes the ecological context in which infants initiate gaze communication to a visual environment characterized by a larger interpersonal distance and, therefore, more objects cluttered between the dyad. The present study has wider implications for the developmental change of shared attention in conjunction with walking development.

Keywords: social interaction, interpersonal distance, eye contact, crawling, walking, second-person perspective, head-mounted eye tracking

\section{INTRODUCTION}

One aim of developmental science is to explain phenomena that occur in infants' everyday lives. Developmental theories often make hypotheses, assumptions, and implications, known as "ecological commitments," about what happens in infants' daily lives outside research contexts (Dahl, 2017). To test or support ecological commitments empirically, it is important to investigate infants' lived experiences in naturalistic environments as well as randomized control tests in experimental rooms. Recent advances in technology and analytical methods have made it possible to evaluate what and how infants see around them or hear in their everyday experience, such as 
their daily language environment (Roy et al., 2015; Bergelson et al., 2019) and visual experience of faces (Jayaraman et al., 2015; Jayaraman and Smith, 2019), hands (Fausey et al., 2016) or objects (Clerkin et al., 2017; Suanda et al., 2019).

Acquisition of walking is one factor that changes how infants see the world in everyday lives because our firstperson perspective visual experiences are shaped by our bodies. Compared with crawling infants, walking infants have higher and more distant visual fields (Kretch et al., 2014). The development of wearable eye trackers for free-moving infants has demonstrated that acquisition of walking changes not only infants' visual exploration but also gaze communication between infant and parent. While moving on a walkway in the laboratory room, walking infants directed their gaze straight ahead at parents in front of them, whereas crawling infants looked down at the floor (Kretch et al., 2014). A similar tendency was confirmed in the situation where both infant and parent could move freely in the laboratory room. Infants with an upright or sitting posture were more likely to look at parent's faces and engage in eye contacts than infants with prone postures (Franchak et al., 2018). These studies suggest that the change in infants' firstperson perspective accompanied by a change in their locomotion or posture affects the frequency of infants' social looks and eye contact.

The question that remains to be answered is how the situation in which gaze communication occurs in daily lives changes along walking development. Unlike gaze behavior directed toward a social stimulus presented on the monitor in the experimental room, daily gaze communication in the real world is embedded in the three-dimensional space. In the space, both infants and parents can move around freely, and many objects are arranged in a complex manner. In such a messy environment, the transition from crawling to walking changes not only infants' locomotion itself, but also infants' interaction with objects and people. Compared to crawlers, walkers move more, see more, play more, and interact more (for reviews, see Adolph and TamisLeMonda, 2014). After the onset of walking, infants take more steps, travel farther distances, and fall less (Adolph et al., 2012). The elevated vantage point of walkers enables them to see distant objects (Kretch et al., 2014), and the hands that become free from supportive functions allow access to and carrying of distant objects (Karasik et al., 2011, 2012; Dosso and Boudreau, 2014). Moreover, walkers are more likely to approach their parents to share objects (Karasik et al., 2011) and make vocalizations and gestures directed to their parents (Clearfield, 2011). Taken together, it is no wonder that the ecological context in which gaze communication occurs may also change as infants' interaction with objects and people changes with walking development.

The aim of the current study is to investigate how the ecological context in which daily gaze communication occurs changes with walking development from the point of view of the spatial arrangement of the infant, the parents, and objects. The interpersonal distance and relative arrangement of objects influence infant-parent gaze communication by interacting with the magnitude of the gesture and the infant's age (Butterworth and Jarrett, 1991; Deák et al., 2000; Flom et al., 2004; Gonseth et al., 2013; Yamamoto et al., 2019). The infant-parent distance modulates the smooth exchange of eye contact and influences the infant's and the parent's social looks differently (Yamamoto et al., 2019). In referential gaze communication, distractor objects in the visual environment often disturb young infants' detection of what their parents refer to, and noticeable gestures from parents are needed to coordinate their visual attention (Butterworth and Jarrett, 1991; Flom et al., 2004). Although the acquisition of walking changes the interaction between infants and objects or people, little is known about the developmental change of such spatial arrangements as that of infants, parents, and objects. In general, gaze communication is the basis of social learning in infants, and it leads to later language development and theory of mind (Brooks and Meltzoff, 2005, 2015). Thus, to understand how the acquisition of walking shapes new opportunities for social learning, it is important to describe the developmental changes in the daily visual environment in which gaze communication occurs between the infant and the parent, that is, the interpersonal distance and the degree of object clutter at the time of gaze communication.

Despite the importance of describing daily gaze communication, few studies have investigated the gaze communication between a free-moving dyad's in everyday life. This is probably due to several methodological considerations. When recording from a third-person perspective, accurate scoring of an infant's gaze behavior is dependent on the complexity of the environment and position of the video camera (Franchak, 2020a), making this method unsuitable for measuring a free-moving dyad's gaze communication. In fact, many previous studies that measured daily gaze communication set infant and parent at a fixed interpersonal distance so that the participants remained visible in the video cameras (e.g., Deák et al., 2014; de Barbaro et al., 2016). One alternative method is recording from the infant's first-person perspective with a wearable eye tracker. Several studies used this method to measure free-moving infants' visual exploration in a laboratory room. However, in previous studies, an experimenter had to walk with infants to prevent infant injury from face-first falls (e.g., Kretch et al., 2014; Franchak et al., 2018; Hoch et al., 2019b). Thus, this method is also not suitable for the purpose of recording daily gaze communication.

One solution to investigating a free-moving dyad's gaze communication in everyday life is recording face-to-face interaction from the parent's first-person perspective, that is, the infant's second-person perspective. A head-mounted camera worn by the infant's social partner allows for measuring eye contact during a live social interaction more reliably and more validly than when using a stationary camera (Edmunds et al., 2017). Moreover, by using this method, we previously recorded a free-moving dyad's daily eye contact scenes longitudinally and demonstrated that interpersonal distance affects the infant's and parent's social looks differently (Yamamoto et al., 2019).

In this study, taking advantage of the recording from the infant's second-person perspective as above, we show how the transition from crawling to walking changes the ecological context in which gaze communication occurs in everyday life. This study is an extension of a previous report (Yamamoto et al., 2019) using the same longitudinal dataset. We investigated the 

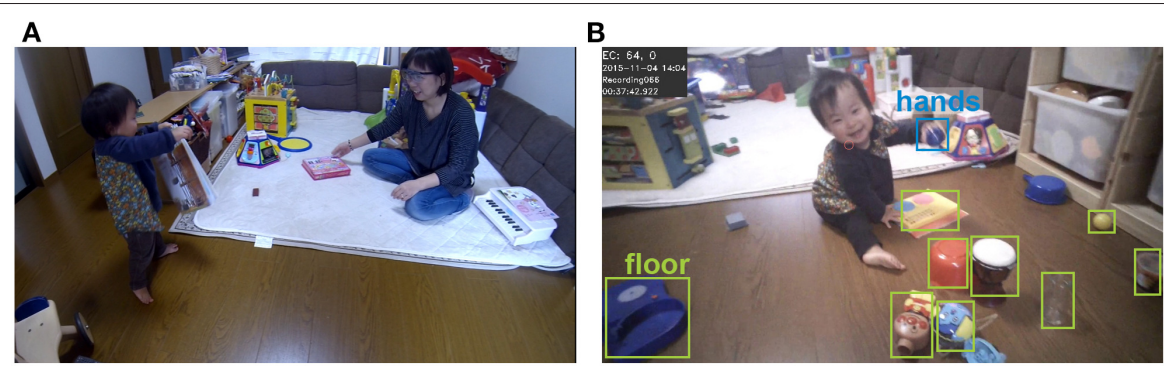

FIGURE 1 | (A) We observed daily infant-parent interaction using head-mounted eye trackers worn by the parents. The infants and parents could move freely in their home environment. (B) Image at the time of eye contact captured from the scene camera of a head-mounted eye tracker worn by a parent. For each eye contact scene, we counted the number of objects on the floor between the dyad (light green) and objects in infant's hands (blue).

developmental change in the spatial arrangement of infants, parents, and objects where gaze communication occurred, focusing on (i) infant-parent distance and (ii) the number of objects between them at the time of eye contact. Regarding the interpersonal distance at the time of eye contact, we predict that the effect of walking development would vary depending on who initiates eye contact with the social partner. After the onset of walking, the elevated vantage point leads infants to see and access distant objects or people (Karasik et al., 2011; Kretch et al., 2014), but there is no such change for parents. If an infant's visual exploration leading to gaze communication is shaped by the infant's first-person perspective view, the interpersonal distance at the time of eye contact would increase with the infant's walking development only for the eye contact the infant, not the parent, initiates. Regarding the number of objects at the time of eye contact, a previous study has showed that walkers are more likely to carry objects and approach their parents to share these objects than are crawlers (Karasik et al., 2011). Thus, we predict that the visual environment between infant and parent will become cluttered with more objects consistent with the infant's walking development. We evaluate the effect of walking development on the number of objects between the dyad while controlling for the effect of interpersonal distance because the number of objects on the floor between the dyad is expected to increase with the interpersonal distance irrespective of the infant's walking development.

\section{MATERIALS AND METHODS}

\subsection{Participants and Data Collecting}

Five healthy, full-term infants (1 male and 4 female; A-E) and their mothers contributed to the present study, beginning when the infant was 10 months old and ending when the infant was 15.5 months of age. All participants were of Japanese ethnicity. This sample was taken from a longitudinal study investigating the effect of interpersonal distance on infant-parent gaze communication by Yamamoto et al. (2019).

We visited each participant's home on alternate weeks and recorded infants' and parents' daily activities from a headmounted eye tracker (Tobii Glasses 2, Tobii Technology) worn by parents for up to $1.5 \mathrm{~h}$ each day. Before every recording, the parent wearing the head-mounted eye tracker was instructed to look at and focus on the center of a card with a blackand-white target held at arm's length, and a calibration was then performed using eye-tracking software (Tobii Glasses Controller). We told parents that we were just interested in the infants' everyday activity and infants and parents could engage in any daily activities, go anywhere in their home and play with any of the available toys (see Figure 1A). After the observation, we measured the infant's face size (between the chin and the eyebrows).

For each observation day, we also recorded infant-parent social interaction with a head-mounted camera (GoPro Hero4, Woodman Labs) worn by H.Y. so that participants could be seen on the head-mounted camera as much as possible. However, due to a malfunction of the battery, we were unable to record videos from the head-mounted camera on several observation days (when infant A was 12.5 months of age, when infant D was 14.5 months of age, and when infant $\mathrm{E}$ was 15.5 months of age).

We could not collect data when infant E was 12 months of age because infant $\mathrm{E}$ was in poor physical health. The mean observation time for each day was $1 \mathrm{~h} 25 \mathrm{~min}$, and the mean total observation time for one infant was $16 \mathrm{~h} 48 \mathrm{~min}$. Extensive details regarding data collection and the age in days for each observation day are included in the report by Yamamoto et al. (2019).

All infants participated with written informed consent from their parents. In addition, for publication of identifying images in an online open-access publication, we obtained informed consent from the parents of the infant, as shown in Figure 1. This research was approved by the ethics review board at the Unit for Advanced Studies of the Human Mind, Kyoto University (27-P-6) and was conducted in accordance with the Helsinki Declaration guidelines and regulations.

\subsection{Data Processing}

Using video recordings from the parent's point of view, we performed five data processing steps (Yamamoto et al., 2019). (i) We coded 3 types of infant locomotion- "crawling," "cruising," and "walking" - with one-zero sampling (Altmann, 1974) for 15 $s$ and calculated the proportion of the infant's walking time to the sum of walking time and crawling time for each observation day. (ii) By checking the parent's perspective video frame by frame, we identified the video frame of each eye contact. The video frame of the eye contact was defined as the video frame 
of the "parent looking at the infant's face," and the infant's gaze was coded as "directed to the parent" by the coders. Coding whether the infant's gaze was directed to the parent from the parent's perspective video was based on Edmunds et al. (2017). We defined continuous eye contact video frames that included glances from either partner for less than $1 \mathrm{~s}$ but no longer than $1 \mathrm{~s}$ as an eye contact bout (EC bout). (iii) By checking which partner initiated the eye contact, we categorized an EC bout as either an infant-led or parent-led EC bout. (iv) We defined an eye contact session (EC session) as a series of EC bouts with short inter-EC-bout intervals and used it as an independent observation unit because EC bouts usually occurred intermittently. (v) The monocular camera generates a one-to-one relationship between the object and the image. Using the video frames of EC bouts, we estimated the interpersonal horizontal distance at the time of the EC bouts from the accelerometer data from the head unit, the focal length, and the real and pixel size of the infant's face (between the chin and the eyebrows). Extensive details regarding data processing were included in the report by Yamamoto et al. (2019).

The aim of the present study was to investigate developmental changes in the spatial arrangement of infants, parents, and objects where daily gaze communication occurs, focusing on infantparent distance and the number of objects between them. With this aim, we newly coded two measures: the proportion of distance category and the number of objects between the dyad.

\subsubsection{Proportion of Distance Category}

To understand the relation between walking development and the infant-parent distance at which gaze communication occurs, it is also necessary to investigate the usual infant-parent distance, which is not limited to the gaze communication context. If an infant's walking development increases the time the infant is far from their parents at various daily contexts, and if gaze communication occurs randomly and irrespective of context, then it is no wonder that the interpersonal distance at the time of eye contact increased with walking development. However, if the developmental change of an infant's first-person perspective shapes the interpersonal distance in face-to-face interactions, then an increase in the interpersonal distance consistent with walking development might occur more clearly in the gaze communication context than in other contexts. To investigate whether the developmental change of the interpersonal distance at the time of eye contact could be simply explained by the developmental change of the usual interpersonal distance, we need to evaluate the usual interpersonal distance for each observation day.

We coded infant-parent distance using the recording from a head-mounted camera worn by H.Y. We coded infant-parent distance into four ordered categories, " $0-0.5 \mathrm{~m}$," "0.5-1.0 m," "1.0-1.5 m," and "1.5 m or more," with instantaneous sampling (Altmann, 1974) for every $30 \mathrm{~s}$. There were some instantaneous samples in which the infants' movement had been constrained by the parent or environmental objects. For example, infants were sometimes put in playpens when the parent did not want to be disrupted by the infants in order to do light housekeeping. Moreover, infants were sometimes held or carried by their parents in social interactions. We did not code such instantaneous samples because infants could not adjust the interpersonal distance. We calculated the proportion of each distance category for each observation day. The second coder independently judged a randomly selected $20 \%$ of the video, with $75 \%$ intercoder agreement $(k a p p a=0.77)$.

\subsubsection{Number of Objects Between Infant and Parent}

Using the head-mounted eye-tracker worn by the parents, we output the first frame of each EC bout and coded the number of objects placed between infant and parent. We counted the number of objects that infants could lift from the floor, such as balls or toys and not tables or sofas. This definition was based on the concepts of "detached objects" and "attached objects" from Gibson (1979). Using this definition, we prioritized foreground, not background, objects (the book on the floor and not the floor itself).

Sometimes, there were EC bouts in which it was difficult to accurately count individual objects, such as balls in a basket. In such situations, multiple objects were nested within another object, and one object could be covered with another object. Because it was difficult to count the exact number of nested objects at the micro level, we counted the macroscopic visual unit as one object. In case of "balls in a basket," we counted all the balls and the basket as one object.

Some objects were sometimes held by the infant or parent. For each EC bout, we counted the number of objects between infant and parent in each of object locations, "on the floor" or "in infant's hands" (see Figure 1B). The second coder independently judged a randomly selected $20 \%$ of the EC bouts with average $81.6 \%$ intercoder agreement (on the floor: $78 \%$; in infant's hands: $85 \%$ ), and the numbers of objects were correlated (on the floor: $r=0.55$; in infant's hands: $r=0.80$ ). We removed three EC bouts $(0.001 \%$ of total EC bouts) because of a difficulty in coding from an image blur, and finally, we analyzed 3135 EC bouts.

\subsection{Data Analysis}

We conducted three main statistical analyses using Bayesian state-space models. The core of the state-space model is a generalized linear mixed model (GLMM) (Baayen, 2008) used to estimate the effects of various explanatory variables on the response variables measured from longitudinal observation data and considering the effects of temporal autocorrelation. Analysis 1 was intended to estimate the effect of walking development on the infant-parent distance at which eye contact occurs. Analysis 2 was intended to estimate the effect of walking development on the usual infant-parent distance, which is not limited to the gaze communication context. Analysis 3 was intended to estimate the effect of walking development on the number of objects between infant and parent. In each analysis, we estimated the coefficient parameters of the explanatory variables. If the parameter estimate of one explanatory variable is positive, it can be interpreted that the response variable increases with the value of the explanatory variable, while controlling for the effects of the other explanatory variables. If the $95 \%$ credible interval of the parameter does not include zero, it can be inferred 
that there is a significant effect, as seen in classic statistical hypothesis testing.

In a previous study, we found that infant-led EC occurs at a greater interpersonal distance than parent-led EC (Yamamoto et al., 2019). The purpose of Yamamoto et al. (2019) was to evaluate the effect of infant-parent distance on daily gaze communication between the dyad, and the response variable was the number of EC bouts within the EC session. Contrary to Yamamoto et al. (2019), the purpose of the current study is to evaluate the effects of an infant's walking development on infantparent distance (Analysis 1) or the number of objects between the dyad (Analysis 3) at the time of eye contact. Although the purpose and response variables were different from those in the previous study, we used the same sample in this study as that used in the previous study. To avoid redetecting the previously reported effects involving the initiator of eye contact, we divided the data into two subsets by the initiator of the EC bouts, and we individually analyzed infant-led EC bouts and parent-led EC bouts in Analysis 1 and Analysis 3, respectively.

In Analysis 1, the response variable was the infant-parent distance of an infant-led EC bout or parent-led EC bout following a lognormal distribution. The explanatory variables were infant age and proportion of infant's walking time for each observation day. Analysis 2 was a kind of mixed ordered logistic regression controlling for the effects of temporal autocorrelation, and the response variable was the proportion of each distance category for each observation day. The explanatory variables were the same as in Analysis 1. In Analysis 3, the response variable was the number of objects on the floor between the dyad or objects in the infant's hands at the time of an infant-led EC bout or parentled EC bout following a Poisson distribution. The explanatory variables were infant age, proportion of infant's walking time for each observation day, and infant-parent distance at each EC bout. To consider differences in the EC session, we set the EC session as a random intercept in Analysis 1. In Analysis 3, we set the EC session as a random intercept for only the analysis of objects on the floor because this setting made the Markov chains convergence difficult for the analysis of objects in the infant's hands. In all analyses, we chose weakly informative priors for the hyperprior of system noise because they helped to stabilize parameter estimates (Gelman et al., 2013). More details on the statistical models are described in the Supplementary Material.

All models were fitted using the Hamiltonian Monte Carlo engine Stan 2.19.2 (Stan Development Team, 2019) in R 3.6.0 ( $\mathrm{R}$ Core Team, 2019). All iterations were set to 11,000 , and burn-in samples were set to 1000 with the number of chains set to four. The values of Rhat for all parameters were below 1.1, indicating convergence across the four chains (Gelman et al., 2013). To check our approach, we simulated the hypothesized data-generating process using the posterior median 50 times, and we iteratively estimated each model in Analysis 1 and Analysis 3.

\section{RESULTS}

Although there were individual differences in motor development, all infants changed their locomotion in daily use from crawling to walking over the longitudinal observation (see Figure $\mathbf{S 1}$ ).

\subsection{Infant-Parent Distance at Which Eye Contact Occurs}

Referring to the $95 \%$ credible interval (CI) of the posterior distributions of the fixed effect parameters (Table 1), the proportion of the infant's walking time had a clearly detected effect on the infant-parent distance at which an infant-led EC bout occurs $($ mean $=0.005,95 \% \mathrm{CI}=[0.0002,0.009]$ ) because the 95\% CI did not include zero. Contrary to an infant-led EC bout, the proportion of the infant's walking time did not have a clearly detected effect on infant-parent distance at which a parent-led EC bout occurs (mean $=0.0007,95 \% \mathrm{CI}=[-0.002,0.004])$. The $95 \%$ CIs of the effect of age on infant-parent distance included zero for both infant-led EC bouts (mean $=-0.053,95 \% \mathrm{CI}=$ $[-0.119,0.010])$ and parent-led EC bouts (mean $=-0.010,95 \%$ $\mathrm{CI}=[-0.053,0.032])$.

Figure 2 shows the longitudinal development of the infantparent distance at which eye contact occurs for a representative infant-parent dyad (see Figure S2). Because the effect of walking time had a positive value only for infant-led EC bouts, the predictions of the infant-parent distance at the time of eye contact increased with the proportion of the infant's walking time only for infant-led EC bouts but not for parent-led EC bouts. We can also confirm this tendency from predictions of the mean infantparent distance at the time of eye contacts for each observation day from all infant-parent dyads (Figure 3).

To check our approach, we simulated new time series data and estimated the parameters of the model with the new data 50 times. Regarding the infant-led EC bouts, the Markov chains converged 49 times, and the 95\% CIs of the posterior distributions of the fixed effect parameters did not include zero 18 times (36.7\%) for the effect of age, and they did not include zero 29 times (59.2\%) for the effect of walking time. Regarding the parent-led EC bouts, the Markov chains converged 50 times, the $95 \%$ CIs of the posterior distributions of the fixed effect parameters did not include zero 5 times (10\%) for the effect of age, and they did not include zero 8 times (16\%) for the effect of walking time.

\subsection{Proportion of Distance Category}

Referring to the 95\% CI of the posterior distributions of the fixed effect parameters (Table 1), both the proportion of infant's walking time $($ mean $=0.005,95 \% \mathrm{CI}=[-0.006,0.014])$ and age (mean $=0.016,95 \% \mathrm{CI}=[-0.131,0.193])$ had no clearly detected effects on the proportion of distance category because the 95\% CIs included zero. This result suggests that the usual infantparent distance that is not limited to the gaze communication context did not change with the proportion of infant's walking time when controlling for the effect of temporal autocorrelation and infant age (see Figure S3).

\subsection{Number of Objects Between Infant and Parent}

Regarding the objects on the floor, referring to the $95 \%$ CIs of the posterior distributions of the fixed effect parameters (Table 1), 
TABLE 1 | The posterior distribution of the parameters of the model.

\begin{tabular}{|c|c|c|c|c|c|}
\hline Analysis & Response variable & Explanatory variable & EAP & $2.5 \%$ & $97.5 \%$ \\
\hline \multirow[t]{4}{*}{ Analysis 1} & Distance (Infant-led EC) & Age & -0.053 & -0.119 & 0.010 \\
\hline & & Walking time & 0.005 & 0.0002 & 0.009 \\
\hline & Distance (Parent-led EC) & Age & -0.010 & -0.053 & 0.032 \\
\hline & & Walking time & 0.0007 & -0.002 & 0.004 \\
\hline \multirow[t]{2}{*}{ Analysis 2} & Proportion of distance category & Age & 0.016 & -0.131 & 0.193 \\
\hline & & Walking time & 0.005 & -0.006 & 0.014 \\
\hline \multirow[t]{12}{*}{ Analysis 3} & Number of objects on the floor & Age & -0.041 & -0.180 & 0.092 \\
\hline & (Infant-led EC) & Walking time & -0.002 & -0.013 & 0.009 \\
\hline & & Distance & 0.944 & 0.750 & 1.15 \\
\hline & Number of objects on the floor & Age & 0.016 & -0.135 & 0.159 \\
\hline & (Parent-led EC) & Walking time & -0.012 & -0.024 & 0.00002 \\
\hline & & Distance & 1.63 & 1.26 & 2.00 \\
\hline & Number of objects in infant's hands & Age & -0.032 & -0.096 & 0.028 \\
\hline & (Infant-led EC) & Walking time & 0.003 & -0.002 & 0.008 \\
\hline & & Distance & -0.051 & -0.176 & 0.068 \\
\hline & Number of objects in infant's hands & Age & -0.024 & -0.096 & 0.042 \\
\hline & (Parent-led EC) & Walking time & 0.001 & -0.004 & 0.006 \\
\hline & & Distance & 0.144 & -0.035 & 0.312 \\
\hline
\end{tabular}

The mean (EAP) and quantiles (2.5\% and 97.5\%) of the posterior distribution are shown.

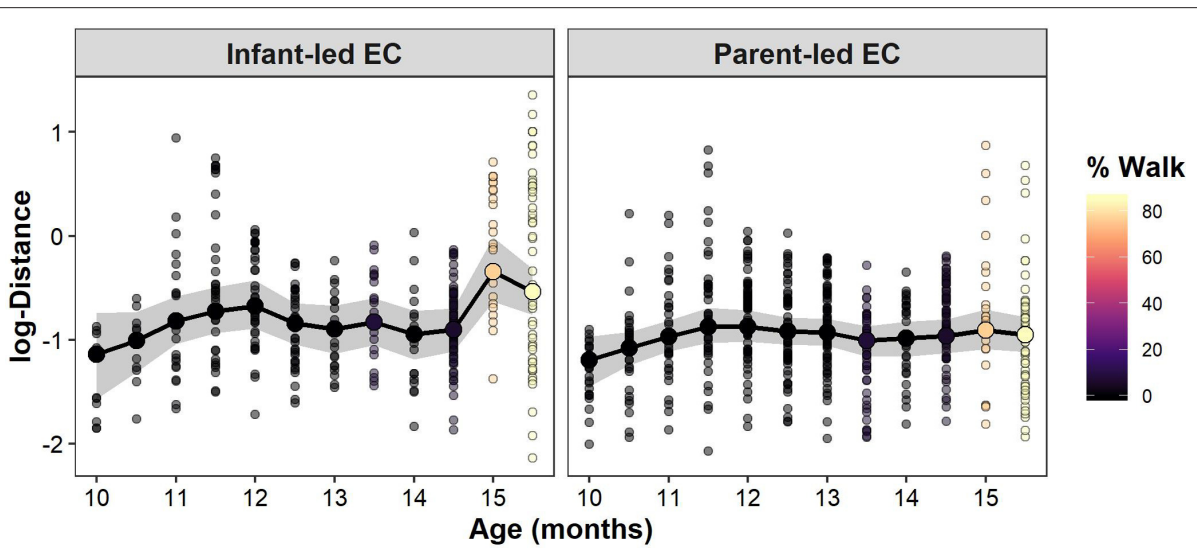

FIGURE 2 | Longitudinal development of interpersonal distance at which infant-led EC bouts (left) occur and interpersonal distance at which parent-led EC bouts (right) occur in one representative infant (infant A). The posterior mean (large colored dots) and 95\% credible interval (gray areas) of the mean interpersonal distance of EC bouts in each observation day are shown. The observed data are represented with small colored dots. The color of the dots represents the proportion of the infant's walking time to the sum of walking time and crawling time for each observation day. Note that the interpersonal distance of each EC bout (meters) is log transformed.

the $95 \%$ CIs of the effect of walking time on the number of objects between the dyad included zero for both infant-led EC bouts (mean $=-0.002,95 \% \mathrm{CI}=[-0.013,0.009])$ and parentled EC bouts (mean $=-0.012,95 \% \mathrm{CI}=[-0.024,0.00002])$. The $95 \%$ CIs of the effect of age also included zero for both infantled EC bouts (mean $=-0.041,95 \% \mathrm{CI}=[-0.180,0.092])$ and parent-led EC bouts (mean $=0.016,95 \% \mathrm{CI}=[-0.135,0.159])$. Infant-parent distance had a clearly detected effect on the number of objects between the dyad for both infant-led EC bouts (mean $=0.944,95 \% \mathrm{CI}=[0.750,1.15])$ and parent-led EC bouts (mean
$=1.63,95 \% \mathrm{CI}=[1.26,2.00])$ because the $95 \% \mathrm{CIs}$ did not include zero.

Regarding the objects in the infant's hands, the 95\% CIs of all effects on the number of objects included zero for both infant-led EC bouts (age: mean $=-0.032,95 \% \mathrm{CI}=[-0.096,0.028]$; walking time: mean $=0.003,95 \% \mathrm{CI}=[-0.002,0.008]$; distance: mean $=$ $-0.051,95 \% \mathrm{CI}=[-0.176,0.068])$ and parent-led EC bouts (age: mean $=-0.024,95 \% \mathrm{CI}=[-0.096,0.042]$; walking time: mean $=$ $0.001,95 \% \mathrm{CI}=[-0.004,0.006]$; distance: mean $=0.144,95 \% \mathrm{CI}$ $=[-0.035,0.312])$. 


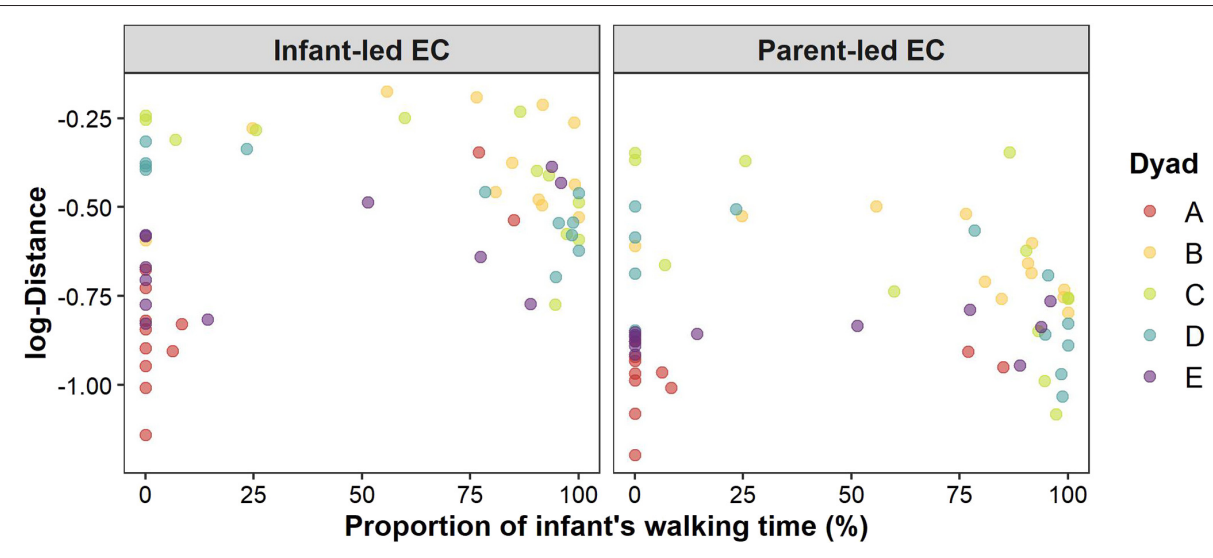

FIGURE 3 | Relationship between the proportion of infant's walking time and the posterior mean of the interpersonal distance (log transformed) at the time of infant-led EC bouts (left) and parent-led EC bouts (right). Each dot represents one observation day. The color of the dots represents each infant-parent dyad.

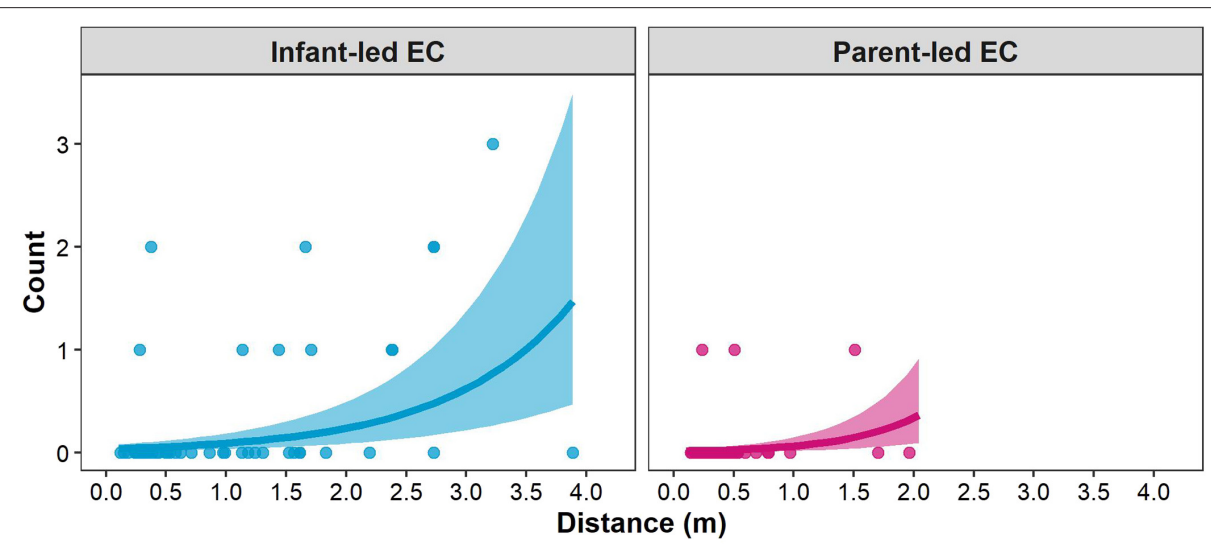

FIGURE 4 | Relationship between the infant-parent distance and number of objects on the floor between the dyad for infant-led EC bouts (left panel; cyan) and parent-led EC bouts (right panel; magenta) on one observation day of one infant (when infant A was 15.5 months of age). The posterior mean (colored lines) and 95\% credible interval (colored areas) of the mean number of objects between the dyad are shown. The colored dots represent the observed data.

Figure 4 shows the predictions for the number of objects on the floor between a dyad for a representative observation day of one infant-parent dyad (see Figures S4, S5). Because the effect of interpersonal distance had a positive value for both infant-led EC bouts and parent-led EC bouts, the predictions of the number of objects on the floor between the dyad increased with interpersonal distance regardless of whether the eye contact was initiated by the infant or the parent.

To check our approach, we simulated new time series data and estimated the parameters of the model with new data 50 times. Regarding the number of objects on the floor at the time of infant-led EC bouts, the Markov chains converged 48 times, and the $95 \%$ CIs of the posterior distributions of the fixed effect parameters did not include zero 0 times $(0 \%)$ for the effect of age, they did not include zero 2 times (4.2\%) for the effect of walking time, and they did not include zero 48 times (100\%) for the effect of interpersonal distance. Regarding the number of objects on the floor at the time of parent-led EC, the Markov chains converged 48 times, and the 95\% CIs of the posterior distributions of the fixed effect parameters did not include zero 3 times $(6.3 \%)$ for the effect of age, they did not include zero 37 times $(77.1 \%)$ for the effect of walking time, and they did not include zero 48 times (100\%) for the effect of interpersonal distance. Regarding the number of objects in the infant's hands at the time of infant-led EC bouts, the Markov chains converged 48 times, and the $95 \%$ CIs of the posterior distributions of the fixed effect parameters did not include zero 4 times $(8.3 \%)$ for the effect of age, they did not include zero 8 times $(16.7 \%)$ for the effect of walking time, and they did not include zero 5 times (10.4\%) for the effect of interpersonal distance. Regarding the number of objects in the infant's hands at the time of parentled EC, the Markov chains converged 47 times, and the 95\% CIs of the posterior distributions of the fixed effect parameters did not include zero 4 times $(8.5 \%)$ for the effect of age, they did not include zero 4 times (8.5\%) for the effect of walking time, and they did not include zero 17 times (36.2\%) for the effect of interpersonal distance. 


\section{DISCUSSION}

This is the first study to describe the ecological contexts in which gaze communication occurs and how it changes with walking development in infant's everyday lives. With eye contact scenes recorded from infant's second-person perspective, we evaluated the longitudinal change in the spatial arrangement of infants, parents, and objects at the timing of eye contacts, focusing on infant-parent distance (Analysis $1 \&$ Analysis 2) and the number of objects between them (Analysis 3).

\subsection{Infant-Parent Distance at Which Eye Contact Occurs}

In Analysis 1, interpersonal distance for infant-led EC bouts increased along with the proportion of infant's walking time. This result suggests that the interpersonal distance at which gaze communication occurs from infants increases with walking development. This result could not be explained by mere developmental change to the usual infant-parent distance, which is not limited to the gaze communication context. In Analysis 2 , the proportion of distance category was not associated with the proportion of infant's walking time, suggesting that the amount of time that infants are farther away from their parents did not change with walking development. Moreover, interpersonal distance for parent-led EC bouts did not show a clear change with the proportion of infant's walking time in Analysis 1. These results suggest that the transition from crawling to walking increases the interpersonal distance at which gaze communication is initiated only for infants and not for parents.

Although previous studies have reported that infant-parent distance or time away from parents increased with motor development or age (Jayaraman et al., 2015; Thurman and Corbetta, 2017, 2019; Hoch et al., 2019a; Jayaraman and Smith, 2019), we could not observe an increase in time away from the parent with walking development or age. There are several possible reasons for the discrepancy between this study and previous studies. First, the size of participants' houses may have limited the possible range of infant-parent distance. Motor development is shaped by social and cultural factors because infants grow up in everyday environments (Adolph and Hoch, 2019). Generally, houses are smaller in Japan than in other countries, and such a cultural difference may lead to no change in infant-parent distance in this study. Second, coarse coding with ordinal scales may have prevented detection of the effects found in previous studies. Third, the small sample size may have made it difficult to detect the effect of infant's walking or age. Depending on the situation for data collection or behavioral measures, it is possible that the daily positioning of the infant and parent has expanded with infant age or walking development. However, using the measure of interpersonal distance at the timing of eye contact, the interpersonal distance at which infants initiated eye contact increased with walking development, while the interpersonal distance at which parents initiated eye contact did not change. Such differences in the development of interpersonal distance at the timing of eye contact cannot be explained simply by the usual interpersonal distance, even if the daily positioning of the dyad expanded with walking development. Taken together, these results suggest that at least the space at which gaze communication is initiated by the infant, not by the parent, expands with infants' walking development.

The current study adds to a growing body of research demonstrating that infants' visual experiences are tied to their posture (Kretch et al., 2014; Franchak et al., 2018). Walking infants have higher and more distant visual fields than do crawling infants (Kretch et al., 2014), and infants' motor costs of social looks are lower when infants are in an upright posture than when they are prone (Franchak et al., 2018). In this study, the increased visibility of parents in high and distant positions may have allowed walking infants to look at their social partners from farther interpersonal distances. Unfortunately, it is difficult to make strong claims about infants' social looking behavior from our results because our data were recorded from infants' secondperson perspective, and we have no data about how infants looked at parents when infants were not visible to their parents. However, considering that infants and parents have a "seeing" and "be seen" relationship at the time of eye contact, this study suggests that infants' visual experience of gaze communication is affected by infants' posture or locomotion.

\subsection{Number of Objects Between Infant and Parent}

In Analysis 3, we investigated whether the number of objects between infants and parents is affected by walking development. Contrary to our prediction, regardless of whether eye contact was initiated by the infant or the parent, the number of objects on the floor between the dyad or objects in the infant's hands did not change with the proportion of infant's walking time when controlling for effects of temporal autocorrelation, infant age, and interpersonal distance at which eye contact occurs.

Previous studies suggest that walkers are more likely to carry objects and approach their parents to share objects than crawlers (Clearfield, 2011; Karasik et al., 2011, 2012). However, taking into account interpersonal distance, this study reveals that there is no indication of the space between the dyad becoming more cluttered with objects when walking develops. This may be because infants' natural act of walking is characterized as exploratory rather than destination directed (Cole et al., 2016; Hoch et al., 2019a,b). During free play, short bouts, curved paths, and omnidirectional steps are prevalent in infants' walking (Lee et al., 2018), and infants' walking often does not end near discernible destinations, such as objects or people (Cole et al., 2016; Hoch et al., 2019a,b). Considering that the prevalence of short bouts and the rarity of destinations persist across the development of walking (Cole et al., 2016; Lee et al., 2018), walkers' carrying behavior may not be characterized as destination directed. Such a characteristic of infants' natural walking might make it difficult to detect the effect of walking time on the number of objects on the floor between the dyad or objects in the infants' hands in this study.

Although the proportion of infant's walking time did not have a clear effect on the number of objects between infants 
and parents, the number of objects on the floor tended to increase with the infant-parent distance regardless of whether eye contact was initiated by the infant or the parent. In daily life, unless objects are concentrated in a particular location in space, it is generally expected that more objects will appear as the interpersonal distance increases. This study does not directly measure the distribution of objects in infants' everyday environment, but this result shows that the number of objects on the floor between the dyad in daily gaze communication is closely related to the positioning of infant and parent. Considering that developmental change in the interpersonal distance at the time of infant-led EC bouts is associated with walking development, these results suggest that as the crawling infant makes the transition to upright locomotion, eye contact initiated by infants occurs in situations that are more distant and with more objects in front of them, which may have profound implication for the development of shared attention.

\subsection{Implication for Shared Attention}

The current study shows that the ecological context in which gaze communication occurs changes with infants' walking development from the point of view of the spatial arrangement of the infant, the parents, and objects. Along with walking development, eye contact from the infants was likely to occur in situations where the infant-parent distance was larger, and therefore, more objects were cluttered on the floor between the dyad. This finding suggests that infants' locomotion or posture dynamically changes the visual environment between the dyads when infants initiate gaze communication.

In this study, we showed that the space at which eye contact was initiated by the infant expanded with infants' walking development, but it may also be related to shared attention in daily face-to-face interaction. Eye contact is an event closely linked to shared attention, which forms a referential triangle of infants, adults, and target objects (Tomasello, 2009). Eye contact encourages the infant's gaze to follow a target object (Senju and Csibra, 2008; Ishikawa and Itakura, 2019), and infants often produce eye contact to initiate joint attention (Mundy et al., 2007). If the expansion of the space at which an infant-led EC bout occurs derives from an embodied factor, such as a change in the infant's first-person visual experience consistent with their motor development, then the visual environment of the shared attention that the infant experiences may also be characterized by a larger interpersonal distance that is more cluttered with objects consistent with the infant's walking development. Such a spatial arrangement of infant, parent, and objects may be associated with shared attention in multiple ways.

First, the arrangement of objects in the infant's first-person perspective may influence the infant's task demand to achieve shared attention. In general, achieving shared attention involves the relative spatial arrangement of the infant, the parent, the target object, and distractors. When we focus on the spatial arrangement of the target object in the infant's first-person perspective, walking development may decrease the infant's task demand for shared attention. By 6 months of age, some infants can follow an adult's head turn toward a target object within the infant's visual field, and, as infants grow, they can follow an adult's gaze to target objects in their periphery and outside of the infants' visual field (Butterworth and Jarrett, 1991; Deák et al., 2000; Flom et al., 2004). If walking development changes the ecological context of shared attention to that similar to a large interpersonal distance so that the parent's face and the target object are simultaneously within the infant's visual field, then the infant might achieve shared attention easily because the parent's gaze can be tracked without the motor cost from tilting the infant's head up or object representation outside of the visual field. On the other hand, when we focus on the spatial arrangement of distractors within an infant's first-person perspective, walking development may increase the infant's task demand for shared attention. Although it depends on the noticeability of the parent's attention-directing gestures (i.e., looking, head turn, and pointing), young infants often fixate on intermediate objects or distractors and fail to engage in shared attention (Butterworth and Jarrett, 1991; Flom et al., 2004). If walking development changes the ecological context of shared attention to that similar to a large interpersonal distance so that many distractors are within the infant's visual field, such a situation might make it difficult to achieve shared attention, especially for young infants.

Second, the arrangement of objects on the floor between the dyad may influence a pathway to achieve shared attention. Recent studies using head-mounted eye trackers worn by infants have reported that there are two pathways to achieve shared attention: the gaze-following pathway and the hand-following pathway ( $\mathrm{Yu}$ and Smith, 2013, 2017a,b). If the visual environment between the dyad at which the shared attention occurs changes with walking development, then the weight of the pathways the infant uses to achieve shared attention may also change. For example, at a large interpersonal distance, infants may use information from parents' gaze direction rather than parents' hand movement to achieve shared attention because there may be many objects that each person in the dyad cannot manually access, and hand-following would not work for sharing attention about such objects. To test these predictions, it would be necessary to investigate developmental change in the ecological context in which shared attention occurs by using head-mounted eye trackers on both infant and parent.

Acquisition of new motor skills instigates and facilitates cascades of change across a range of domains; this is known as a developmental cascade (for reviews, see Campos et al., 2000; Anderson et al., 2013; Adolph and Hoch, 2019; Franchak, 2020b). A particularly intriguing developmental cascade traces walking experience to language development. The onset of walking is associated with increases in infants' receptive and productive vocabulary (Walle and Campos, 2014). Although the causal mechanism is not fully identified yet, previous studies have focused on social interaction as a factor that links walking to language. Walkers more frequently retrieve (Dosso and Boudreau, 2014), carry, and share distal objects (Clearfield, 2011; Karasik et al., 2011, 2012), and parents provide different verbal responses to walkers compared with crawlers (Karasik et al., 2014). However, although shared attention is closely related to later language development (Brooks and Meltzoff, 2005; 
Okumura et al., 2017), there is no behavioral study investigating the relation between walking development and shared attention in everyday life (but see Walle, 2016). To reveal the causal mechanism of the developmental cascade set off by walking, it would be necessary to investigate the relation between walking development and shared attention, taking into account the gaze communication space in further study.

\subsection{Limitations and Conclusions}

There are several limitations to this study. One limitation is its small sample size as our data came from only five dyads. There are many previous studies that employ small sample sizes but analyze high-density data in language, motor, and social development (Thelen et al., 1993; Yoshida and Smith, 2008; Franchak et al., 2011; Yu and Smith, 2012; Roy et al., 2015; Clerkin et al., 2017; Suanda et al., 2019). This is especially true in headmounted eye tracking studies, as the time-intensive, frame-byframe scoring typically leads to modest sample sizes (Franchak, 2020a), and this study is no exception. Although our dense set of longitudinal recordings provides useful information to understand the developmental process of natural gaze behavior, establishing the generality of our results will require more evidence. Another limitation comes from our method. It is difficult to draw any conclusion about infants' own social looking behavior because our data were recorded from infants' secondperson perspective. In addition, the implications for shared attention must be considered as hypotheses to be tested because our results are descriptive and correlational.

In spite of the limitations above, this study shows how the spatial arrangement of the infant, the parent, and objects where gaze communication occurs changes with walking development in everyday life. The transition from crawling to walking changes the ecological context in which infants initiate eye contact to a visual environment characterized by a large infant-parent distance and more objects cluttered between the dyad. Infants' exploration is closely tied with their posture or motor skills, and the exploratory experiences in everyday life are assumed to mediate developmental cascades (Franchak, 2020b). Although many developmental theories have emphasized the role of infants' experience in infant development for a long time, direct measurement of infants' daily experiences is rare (Dahl, 2017). By recording daily face-to-face interaction from infants' secondperson perspective, we found longitudinal change in free-moving dyads' gaze communication in everyday life. Further studies describing daily gaze communication from infants' secondperson perspective as well as infants' first-person perspective may shed light on how new motor skills provide infants with new opportunities for learning in their lived experiences.

\section{REFERENCES}

Adolph, K. E., Cole, W. G., Komati, M., Garciaguirre, J. S., Badaly, D., Lingeman, J. M., et al. (2012). How do you learn to walk? Thousands of steps and dozens of falls per day. Psychol. Sci. 23, 1387-1394. doi: 10.1177/0956797612 446346

\section{DATA AVAILABILITY STATEMENT}

The datasets and the codes used for the models and graphs are available in the GitHub repository (https:/github.com/ dororo1225/GazeCommunication2).

\section{ETHICS STATEMENT}

The studies involving human participants were reviewed and approved by the ethics review board at the Unit for Advanced Studies of the Human Mind, Kyoto University. Written informed consent to participate in this study was provided by the participants' legal guardian/next of kin. Written informed consent was obtained from the individual(s), and minor(s)' legal guardian/next of kin, for the publication of any potentially identifiable images or data included in this article.

\section{AUTHOR CONTRIBUTIONS}

HY designed the study, collected field data, carried out the analysis, and drafted the manuscript. AS and SI helped with data collection and drafting the manuscript.

\section{FUNDING}

This study was supported by Grants-in-Aid for Scientific Research from the Japan Society for the Promotion of Science (grant nos. 14J04047, 26280048, 25245067, and 16H06301).

\section{ACKNOWLEDGMENTS}

This study was made possible by the enthusiastic cooperation of the infants and their families. We sincerely thank them. We also thank N. Furuhata and R. Mizobata for their technical support. A. Sano, R. Yamaguchi, and K. Inada assisted us in data coding. We also thank the reviewers and M. Ishikawa and Y. Okumura for their helpful comments on earlier drafts of this paper. This study was supported by the Center for Baby Science, Doshisha University (Joint Usage/Research Center for Baby Science accredited by the Ministry of Education, Culture, Sports, Science and Technology).

\section{SUPPLEMENTARY MATERIAL}

The Supplementary Material for this article can be found online at: https://www.frontiersin.org/articles/10.3389/fpsyg. 2019.02987/full\#supplementary-material

Adolph, K. E., and Hoch, J. E. (2019). Motor development: embodied, embedded, enculturated, and enabling. Annu. Rev. Psychol. 70, 141-164. doi: 10.1146/annurev-psych-010418-102836

Adolph, K. E., and Tamis-LeMonda, C. S. (2014). The costs and benefits of development: the transition from crawling to walking. Child Dev. Perspect. 8, 187-192. doi: 10.1111/cdep.12085 
Altmann, J. (1974). Observational study of behavior: sampling methods. Behaviour 49, 227-266. doi: 10.1163/156853974X00534

Anderson, D. I., Campos, J. J., Witherington, D. C., Dahl, A., Rivera, M., He, M., et al. (2013). The role of locomotion in psychological development. Front. Psychol. 4:440. doi: 10.3389/fpsyg.2013.00440

Baayen, R. (2008). Analyzing Linguistic Data: A Practical Introduction to Statistics Using R. Cambridge, UK: Cambridge University Press.

Bergelson, E., Amatuni, A., Dailey, S., Koorathota, S., and Tor, S. (2019). Day by day, hour by hour: naturalistic language input to infants. Dev. Sci. 22:e12715. doi: $10.1111 /$ desc. 12715

Brooks, R., and Meltzoff, A. N. (2005). The development of gaze following and its relation to language. Dev. Sci. 8, 535-543. doi: $10.1111 / j .1467-7687.2005 .00445 . x$

Brooks, R., and Meltzoff, A. N. (2015). Connecting the dots from infancy to childhood: a longitudinal study connecting gaze following, language, and explicit theory of mind. J. Exp. Child Psychol. 130, 67-78. doi: 10.1016/j.jecp.2014.09.010

Butterworth, G., and Jarrett, N. (1991). What minds have in common is space: spatial mechanisms serving joint visual attention in infancy. Brit. J. Dev. Psychol. 9, 55-72. doi: 10.1111/j.2044-835X.1991.tb00862.x

Campos, J. J., Anderson, D. I., Barbu-Roth, M. A., Hubbard, E. M., Hertenstein, M. J., and Witherington, D. (2000). Travel broadens the mind. Infancy 1, 149-219. doi: 10.1207/S15327078IN0102_1

Clearfield, M. W. (2011). Learning to walk changes infants' social interactions. Infant Behav. Dev. 34, 15-25. doi: 10.1016/j.infbeh.2010.04.008

Clerkin, E. M., Hart, E., Rehg, J. M., Yu, C., and Smith, L. B. (2017). Real-world visual statistics and infants' first-learned object names. Philos. Trans. R. Soc. B Biol. Sci. 372:20160055. doi: 10.1098/rstb.2016.0055

Cole, W. G., Robinson, S. R., and Adolph, K. E. (2016). Bouts of steps: the organization of infant exploration. Dev. Psychobiol. 58, 341-354. doi: $10.1002 /$ dev. 21374

Dahl, A. (2017). Ecological commitments: why developmental science needs naturalistic methods. Child Dev. Perspect. 11, 79-84. doi: 10.1111/cdep.12217

de Barbaro, K., Johnson, C. M., Forster, D., and Deák, G. O. (2016). Sensorimotor decoupling contributes to triadic attention: a longitudinal investigation of mother-infant-object interactions. Child Dev. 87, 494-512. doi: $10.1111 /$ cdev. 12464

Deák, G. O., Flom, R. A., and Pick, A. D. (2000). Effects of gesture and target on 12- and 18-month-olds' joint visual attention to objects in front of or behind them. Dev. Psychol. 36, 511-523. doi: 10.1037/0012-1649.36.4.511

Deák, G. O., Krasno, A. M., Triesch, J., Lewis, J., and Sepeta, L. (2014). Watch the hands: infants can learn to follow gaze by seeing adults manipulate objects. Dev. Sci. 17, 270-281. doi: 10.1111/desc. 12122

Dosso, J. A., and Boudreau, J. P. (2014). Crawling and walking infants encounter objects differently in a multi-target environment. Exp. Brain Res. 232, 30473054. doi: 10.1007/s00221-014-3984-z

Edmunds, S. R., Rozga, A., Li, Y., Karp, E. A., Ibanez, L. V., Rehg, J. M., et al. (2017). Brief report: using a point-of-view camera to measure eye gaze in young children with autism spectrum disorder during naturalistic social interactions: a pilot study. J. Autism Dev. Disord. 47, 898-904. doi: 10.1007/s10803-0163002-3

Fausey, C. M., Jayaraman, S., and Smith, L. B. (2016). From faces to hands: changing visual input in the first two years. Cognition 152, 101-107. doi: 10.1016/j.cognition.2016.03.005

Flom, R., Deák, G. O., Phill, C. G., and Pick, A. D. (2004). Nine-month-olds' shared visual attention as a function of gesture and object location. Infant Behav. Dev. 27, 181-194. doi: 10.1016/j.infbeh.2003.09.007

Franchak, J. M. (2020a). "Looking with the head and eyes," in Perception as Information Detection: Reflections on Gibson's Ecological Approach to Visual Perception, eds J. Wagman and J. Blau (New York, NY: Routledge), 205-221.

Franchak, J. M. (2020b). The ecology of infants' perceptual-motor exploration. Curr. Opin. Psychol. 32, 110-114. doi: 10.1016/j.copsyc.2019.06.035

Franchak, J. M., Kretch, K. S., and Adolph, K. E. (2018). See and be seen: infant-caregiver social looking during locomotor free play. Dev. Sci. 21:e12626. doi: $10.1111 /$ desc. 12626

Franchak, J. M., Kretch, K. S., Soska, K. C., and Adolph, K. E. (2011). Headmounted eye tracking: a new method to describe infant looking. Child Dev. 82, 1738-1750. doi: 10.1111/j.1467-8624.2011.01670.x
Gelman, A., Carlin, J. B., Rubin, D. B., Vehtari, A., Dunson, D. B., and Stern, H. S. (2013). Bayesian Data Analysis, 3rd Edn. London: Chapman \& Hall/CRC.

Gibson, J. J. (1979). The Ecological Approach to Visual Perception. Boston, MA: Houghton Mifflin.

Gonseth, C., Vilain, A., and Vilain, C. (2013). An experimental study of speech/gesture interactions and distance encoding. Speech Commun. 55, $553-$ 571. doi: 10.1016/j.specom.2012.11.003

Hoch, J. E., O'Grady, S. M., and Adolph, K. E. (2019a). It's the journey, not the destination: Locomotor exploration in infants. Dev. Sci. 22:e12740. doi: $10.1111 /$ desc. 12740

Hoch, J. E., Rachwani, J., and Adolph, K. E. (2019b). Where infants go: real-time dynamics of locomotor exploration in crawling and walking infants. Child Dev. doi: 10.1111/cdev.13250. [Epub ahead of print].

Ishikawa, M., and Itakura, S. (2019). Physiological arousal predicts gaze following in infants. Proc. R. Soc. B Biol. Sci. 286:20182746. doi: 10.1098/rspb.2018.2746

Jayaraman, S., Fausey, C. M., and Smith, L. B. (2015). The faces in infantperspective scenes change over the first year of life. PLoS ONE 10:e0123780. doi: 10.1371/journal.pone.0123780

Jayaraman, S., and Smith, L. B. (2019). Faces in early visual environments are persistent not just frequent. Vis. Res. 157, 213-221. doi: 10.1016/j.visres.2018.05.005

Karasik, L. B., Adolph, K. E., Tamis-LeMonda, C. S., and Zuckerman, A. L. (2012). Carry on: spontaneous object carrying in 13-month-old crawling and walking infants. Dev. Psychol. 48, 389-397. doi: 10.1037/a0026040

Karasik, L. B., Tamis-LeMonda, C. S., and Adolph, K. E. (2011). Transition from crawling to walking and infants' actions with objects and people. Child Dev. 82, 1199-1209. doi: 10.1111/j.1467-8624.2011.01595.x

Karasik, L. B., Tamis-LeMonda, C. S., and Adolph, K. E. (2014). Crawling and walking infants elicit different verbal responses from mothers. Dev. Sci. 17, 388-395. doi: 10.1111/desc.12129

Kretch, K. S., Franchak, J. M., and Adolph, K. E. (2014). Crawling and walking infants see the world differently. Child Dev. 85, 1503-1518. doi: $10.1111 /$ cdev.12206

Lee, D. K., Cole, W. G., Golenia, L., and Adolph, K. E. (2018). The cost of simplifying complex developmental phenomena: a new perspective on learning to walk. Dev. Sci. 21:e12615. doi: 10.1111/desc.12615

Mundy, P., Block, J., Delgado, C., Pomares, Y., Van Hecke, A. V., and Parlade, M. V. (2007). Individual differences and the development of joint attention in infancy. Child Dev. 78, 938-954. doi: 10.1111/j.1467-8624.2007.01042.x

Okumura, Y., Kanakogi, Y., Kobayashi, T., and Itakura, S. (2017). Individual differences in object-processing explain the relationship between early gaze-following and later language development. Cognition 166, 418-424. doi: 10.1016/j.cognition.2017.06.005

R Core Team (2019). R: A Language and Environment for Statistical Computing. Vienna: R Foundation for Statistical Computing.

Roy, B. C., Frank, M. C., DeCamp, P., Miller, M., and Roy, D. (2015). Predicting the birth of a spoken word. Proc. Natl. Acad. Sci. U.S.A. 112, 12663-12668. doi: 10.1073/pnas.1419773112

Senju, A., and Csibra, G. (2008). Gaze following in human infants depends on communicative signals. Curr. Biol. 18, 668-671. doi: 10.1016/j.cub.2008. 03.059

Stan Development Team (2019). RStan: The R Interface to Stan. R package version 2.19.2. Available online at: https://cran.r-project.org/web/packages/ rstan/citation.html

Suanda, S. H., Barnhart, M., Smith, L. B., and Yu, C. (2019). The signal in the noise: the visual ecology of parents' object naming. Infancy 24, 455-476. doi: $10.1111 /$ infa. 12278

Thelen, E., Corbetta, D., Kamm, K., Spencer, J. P., Schneider, K., and Zernicke, R. F. (1993). The transition to reaching: mapping intention and intrinsic dynamics. Child Dev. 64, 1058-1098. doi: 10.2307/1131327

Thurman, S. L., and Corbetta, D. (2017). Spatial exploration and changes in infant-mother dyads around transitions in infant locomotion. Dev. Psychol. 53, 1207-1221. doi: 10.1037/dev0000328

Thurman, S. L., and Corbetta, D. (2019). Changes in posture and interactive behaviors as infants progress from sitting to walking: a longitudinal study. Front. Psychol. 10:822. doi: 10.3389/fpsyg.2019.00822

Tomasello, M. (2009). The Cultural Origins of Human Cognition. Cambridge, MA: Harvard University Press. 
Walle, E. A. (2016). Infant social development across the transition from crawling to walking. Front. Psychol. 7:960. doi: 10.3389/fpsyg.2016.00960

Walle, E. A., and Campos, J. J. (2014). Infant language development is related to the acquisition of walking. Dev. Psychol. 50, 336-348. doi: 10.1037/a0033238

Yamamoto, H., Sato, A., and Itakura, S. (2019). Eye tracking in an everyday environment reveals the interpersonal distance that affords infantparent gaze communication. Sci. Rep. 9:10352. doi: 10.1038/s41598-019-4 6650-6

Yoshida, H., and Smith, L. B. (2008). What's in view for toddlers? Using a head camera to study visual experience. Infancy 13, 229-248. doi: $10.1080 / 15250000802004437$

Yu, C., and Smith, L. B. (2012). Embodied attention and word learning by toddlers. Cognition 125, 244-262. doi: 10.1016/j.cognition.2012.06.016

$\mathrm{Yu}, \mathrm{C}$., and Smith, L. B. (2013). Joint attention without gaze following: human infants and their parents coordinate visual attention to objects through eyehand coordination. PLoS ONE 8:e79659. doi: 10.1371/journal.pone.0079659
Yu, C., and Smith, L. B. (2017a). Hand-eye coordination predicts joint attention. Child Dev. 88, 2060-2078. doi: 10.1111/cdev.12730

Yu, C., and Smith, L. B. (2017b). Multiple sensory-motor pathways lead to coordinated visual attention. Cogn. Sci. 41, 5-31. doi: 10.1111/cogs.12366

Conflict of Interest: The authors declare that the research was conducted in the absence of any commercial or financial relationships that could be construed as a potential conflict of interest.

Copyright (๑) 2020 Yamamoto, Sato and Itakura. This is an open-access article distributed under the terms of the Creative Commons Attribution License (CC BY).

The use, distribution or reproduction in other forums is permitted, provided the original author(s) and the copyright owner(s) are credited and that the original publication in this journal is cited, in accordance with accepted academic practice. No use, distribution or reproduction is permitted which does not comply with these terms. 


\section{OPEN ACCESS}

Edited by:

Antonio lannaccone,

Université de Neuchâtel, Switzerland

Reviewed by:

Carlos Fernando Valenzuela,

University of New Mexico,

United States

Benjamin Dering,

University of Stirling, United Kingdom

*Correspondence:

Alberto Granato

alberto.granato@unicatt.it

Specialty section:

This article was submitted to Developmental Psychology,

a section of the journal

Frontiers in Psychology

Received: 30 October 2019 Accepted: 09 January 2020

Published: 29 January 2020

Citation:

Granato A (2020) The

Transgenerational Consequences of the Interaction Between Humans and

Molecules: Alcohol as a Cultural Artifact. Front. Psychol. 11:61. doi: 10.3389/fpsyg.2020.00061

\section{The Transgenerational}

\section{Consequences of the Interaction Between Humans and Molecules: Alcohol as a Cultural Artifact}

\author{
Alberto Granato* \\ Department of Psychology, Catholic University of the Sacred Heart, Milan, Italy
}

Keywords: alcohol, addiction, intellectual disability, dopamine, development, plasticity, neurons, fetal alcohol spectrum disorders

\author{
With drinking, I cancel all my troubles; \\ What does it matter if I am poor? \\ When I drink I am as rich as the rich Croesus. \\ I really want to sing \\ while I'm lying down, crowned with ivy. \\ Here: I am the master of the world \\ and if you want, o soldier. \\ goes to war too. \\ When you have fallen, pierced, \\ I will be drunk, yes, but far more alive than you
}

(Anacreon, 5th cent. B.C.).

\section{INTRODUCTION}

This paper aims to offer some insights on the concept of socio-materiality from the perspective of neuroscientific research. The example that will be proposed is that of the neuropsychological effects of alcohol abuse at both individual and intergenerational levels.

Alcohol consumption has always been a case of construction and use of cultural artifacts. Neuroscientific studies related to the effects on the brain and behavior of a molecule (ethanol) transformed into consumer objects (alcohol) can profitably be integrated with psychosocial studies on the role of context and social practices in the predisposition to alcohol use/abuse to understand how the encounter with a socio-material element of the experience-alcohol-impacts on psychological development in the life span.

Specifically, with respect to the wide constellation of psychological phenomena related to the "galaxy" of alcohol consumption/abuse, some considerations will be offered on the fetal alcohol spectrum disorders (FASD). In fact, it is grounded in particular socio-cultural situations such as those typically connected to consumption during pregnancy and is one of the main causes of intellectual disability of the offspring; furthermore it also longitudinally predisposes to alcohol abuse in adolescence, enhancing the already strong social pushes to consumption of substances in that age of life also thanks to social alibis such as socializing disinhibition. In short, a vicious circle that must be interrupted, is rooted in the body, affects the mind, and costs to society. At the center of this vicious circle, a cultural artifact that, like many of the socio-material objects of our experience, loses its neutral character depending on the individual and social uses it allows (think of the pervasive debate on the various forms of technological addiction: Milani et al., 2018). 


\section{THE BURDEN OF EARLY ALCOHOL EXPOSURE}

Unlike most drugs acting on our brain, ethanol, usually referred to as alcohol, when considered under the chemical point of view, is a quite simple molecule $\left(\mathrm{CH}_{3}-\mathrm{CH}_{2} \mathrm{OH}\right)$. This might explain why its well-known effect on the central nervous system has been long attributed to a non-specific interaction with the cell membrane of neurons. The neuroscientists now know that alcohol can interact in a specific way with the two main central neurotransmitters, modulating positively some GABA receptors and negatively some glutamate receptors (Lovinger et al., 1990; Weiner and Valenzuela, 2006). Furthermore, other neurotransmitter receptors, including those for the opioids and dopamine, as well as several voltage-gated ion channels, mediate the effects of alcohol on neurons (see Abrahao et al., 2017, for review). Therefore, despite its straight chemical structure, the interplay of alcohol and brain is definitely complex.

Drinking the first glass of wine during an adolescents' party can be an amazing experience, but for a few people the long-lasting outcomes of this encounter may eventually represent a dramatic devastation of their lives. Everything is made even more complicated by the fact that in most Western countries alcoholic beverages are legal, socially accepted, and belong to the consolidated culture. Moreover, new contexts, such as the social media, can boost alcohol consumption in young people (Hendriks et al., 2018).

For alcohol drinkers, the different periods of the lifespan matter, the most striking instance being maternal alcohol consumption during gestation. Drinking during pregnancy harms the brain development of the offspring and can result in FASD, one of the leading non-genetic causes of intellectual disability. The economic and social impact of alcohol misuse during pregnancy is dramatic and the annual cost for children affected by FASD exceeds that of other serious conditions, such as autism (Greenmyer et al., 2018).

Alcohol consumption during pregnancy is underreported in questionnaires (e.g., Morini et al., 2013) and FASD is underdiagnosed, especially in some countries (Vagnarelli et al., 2011) and in selected groups, such as adopted children (Bakhireva et al., 2018). Furthermore, although some authors think that prevention efforts should be devoted only to women with heavy drinking habits (Hatfield, 1985), there is compelling evidence, coming from both human and experimental studies, that even moderate or "social" maternal drinking can permanently impair offspring's cognitive functions (Olson et al., 1997; Valenzuela et al., 2012). Flak and coworkers, after carrying out a meta-analysis on the effects of different levels of prenatal alcohol exposure, conclude that "there is no known safe amount of alcohol to consume while pregnant" (Flak et al., 2014). Not to mention that many other substances of abuse, including cocaine, can negatively affect the brain development following exposure during critical gestational periods (reviewed in Ross et al., 2015). Finally, the co-exposure to more than one substance of abuse, such as alcohol and nicotine, can have a detrimental cumulative or synergic effect on the offspring's brain and cognitive function (e.g., Rivkin et al., 2008; Gautam et al., 2015). This is enough to warn the general public and the policy-maker about the risk of exposing the fetus to harmful molecules.

\section{A PRIMING ROLE OF THE EARLY ALCOHOL EXPERIENCE?}

But there's something else to be worried about. Adolescents and adults exposed to alcohol during fetal life show an increased risk of becoming addicted to alcohol and other drugs (e.g., Baer et al., 2003; Alati et al., 2006), thus perpetuating the damage in a kind of transgenerational self-sustaining vicious circle (Figure 1). One can argue that the higher risk of children of alcoholics is the consequence of several social, environmental, and genetic factors, not necessarily related to in utero exposure (Johnson and Leff, 1999). In addition, the intellectual disability per se might represent a risk factor for developing a substance abuse disorder (Carroll Chapman and $\mathrm{Wu}, 2012$ ).

However, Yates et al. (1998) carried out a study on adoptees, ruling out several potential confounding variables, and concluded that fetal alcohol exposure increases the risk of later drug dependence. Many studies based on experimental reproductions of FASD in rodents helped to answer the question whether early alcohol is directly responsible for the development of drug seeking behavior during adulthood (see, Spear and Molina, 2005, for review). Recently, Wang et al. (2019), using conditioned place preference and self-administration paradigms ${ }^{1}$, demonstrated that prenatal ethanol increases the risk of psychostimulant addiction in adult rats. Therefore, a "priming" role exerted by early alcohol exposure should be taken into account when dealing with the higher risk of drug dependence in young adults affected by FASD. This is not surprising: even after experiencing something of apparent negligible significance, our neurons will never be the same anymore. In a word, neurons are plastic. The neurobiological counterpart of neuronal plasticity was first described by Bliss and Lomo in their seminal paper published in 1973 and is represented by a long lasting increase of synaptic efficacy following repetitive stimulation of glutamatergic hippocampal synapses (Bliss and Lomo, 1973). The observation of this phenomenon, referred to as long term potentiation (LTP), paved the way for the subsequent work on brain plasticity. Many studies pointed out that plasticity can be a good friend, but also a foe, since several neuropsychiatric disorders, including FASD, are characterized by a maladaptive plastic remodeling of neural circuits, and/or by a change of their plastic capacity (see Cohen et al., 2017). On this line of evidence, the permanent impairment

\footnotetext{
${ }^{1}$ Two paradigms largely used in experimental behavioral studies of addiction. The conditioned place preference is based on the observation that addicted animals (usually rodents) spend more time in one of two places in which they were given the drug during conditioning, compared to the other, in which they received only the vehicle (i.e., saline). In the self-administration protocol used in the cited study (Wang et al., 2019), animals were trained to press a lever to receive intravenous injection of the psychostimulant drug. Addicted animals self-administered more drug infusions than controls.
} 


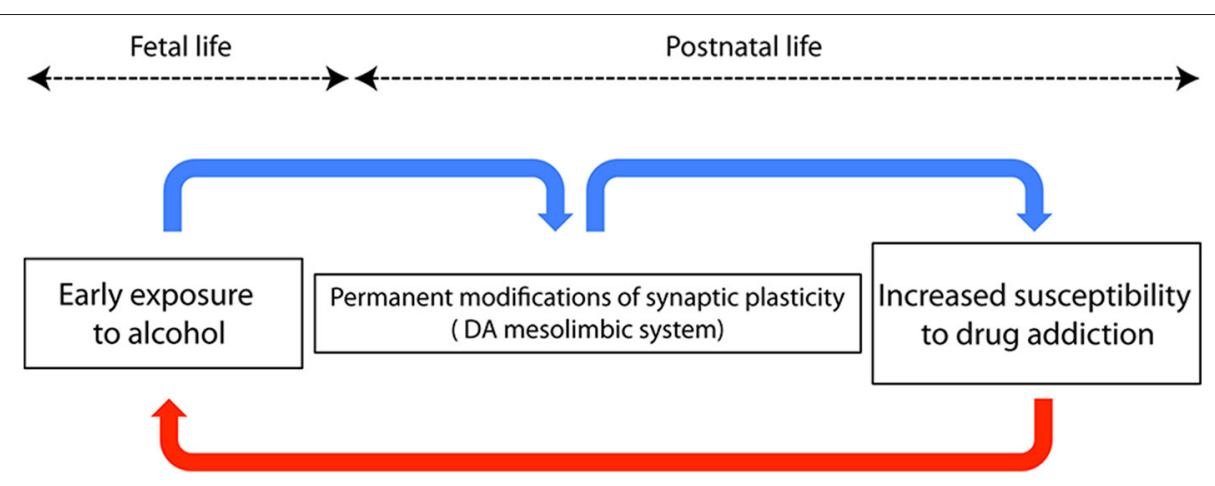

FIGURE 1 | Schematic diagram showing a neurobiological hypothesis to explain the trangenerational self-sustaining circle of drug addiction. Blue arrows represent connections among events occurring in the same individual, while the red arrow represents transgenerational events.

of dendritic calcium electrogenesis observed in cortical neurons after early exposure to ethanol impacts synaptic plasticity, thus accounting for the FASD-related learning disabilities (Granato et al., 2012). Worth mentioning here, the neurobiological basis of addiction is currently considered a sort of "wrong" plasticity, or "excessive" memory of drug experience, occurring in the dopaminergic mesolimbic circuit, the reward processing area of the brain (see Kauer and Malenka, 2007). In utero exposure to alcohol triggers widespread death of neurons (Olney, 2014), whereas surviving cells undergo massive, possibly maladaptive, plastic adjustments, often caused by the same signaling cascade mediating apoptosis (Granato and Dering, 2018). The reward system itself is altered and its plastic responses are persistently modified, as demonstrated by the enhanced excitatory synaptic strength of dopaminergic neurons of the mesolimbic system in adult rats exposed to ethanol during prenatal life (Hausknecht et al., 2015). Considering the role of the dopaminergic system in the genesis of drug addiction, this finding can provide a mechanistic explanation for the increased risk of drug dependence in individuals who experienced an early exposure to ethanol (Figure 1). Other structures known to be involved in addictive behavior, such as the medial prefrontal cortex and the amygdala, are also affected by early exposure to alcohol and might contribute to generate drug dependence during later life (Baculis et al., 2015; Sharp, 2017; Cantacorps et al., 2019). Permanent consequences of early contacts with alcohol can be also explained by epigenetic mechanisms, i.e., by the long-lasting chemical modifications of DNA, some of which are known to be induced by ethanol (e.g., Mead and Sarkar, 2014; Cobben et al., 2019). Epigenetic modifications have also been demonstrated to be responsible for the transgenerational transmission of fetal alcohol effects through the male germline (Sarkar, 2016; Abbott et al., 2018), thus accounting for the paternal contribution to FASD (Abel, 2004).

Finally, prenatal alcohol can increase susceptibility to substance abuse via indirect mechanisms. For instance, FASD are characterized by a higher vulnerability to stress, depression/anxiety disorders (Hellemans et al., 2010), and aberrant pain sensitivity (Sanchez et al., 2017).

\section{CONCLUDING REMARKS}

The key role played by the socioeconomic context and by education in the genesis of alcoholism cannot be underestimated (Boardman et al., 2001; Newton and Lee, 2019). Nevertheless, nature (i.e., neural circuits) and nurture (i.e., environmental context) are strictly interdependent, and can interact in such a way that the former is deeply modified by the latter. Eventually, in case of irreversible circuit changes, even the most refined attempts to improve the environmental conditions may result ineffective. This prompts the neuroscientist to search new therapeutic strategies to counteract the permanent and detrimental plastic changes induced by harmful environmental factors.

Considering alcohol as a cultural artifact with the profound implications for the body here described can lead to a profitable integration between the studies conducted by the neuroscientific and psychosocial perspectives, providing each of them with the opportunity to understand features that would escape from an unintegrated view.

We define alcohol abuse as a phenomenon at risk of intergenerational transmission. In this regard, the psychosocial view offers the possibility of understanding when and in which social and contextual framework the interaction with the molecule takes place; of this same interaction the neuroscientific view can provide a detailed comprehension of the specific mechanisms and their medium and long term consequences from the cerebral point of view.

\section{AUTHOR CONTRIBUTIONS}

The author confirms being the sole contributor of this work and has approved it for publication.

\section{ACKNOWLEDGMENTS}

The author gratefully acknowledges Prof. Antonella Marchetti for helpful suggestions during the preparation of the manuscript. 


\section{REFERENCES}

Abbott, C. W., Rohac, D. J., Bottom, R. T., Patadia, S., and Huffman, K. J. (2018). prenatal ethanol exposure and neocortical development: a transgenerational model of FASD. Cereb. Cortex 28, 2908-2921. doi: 10.1093/cercor/bhx168

Abel, E. (2004). Paternal contribution to fetal alcohol syndrome. Addict. Biol. 9, 127-133. doi: 10.1080/13556210410001716980

Abrahao, K. P., Salinas, A. G., and Lovinger, D. M. (2017). Alcohol and the brain: neuronal molecular targets, synapses, and circuits. Neuron 96, 1223-1238. doi: 10.1016/j.neuron.2017.10.032

Alati, R., Al Mamun, A., Williams, G. M., O'Callaghan, M., Najman, J. M., and Bor, W. (2006). In utero alcohol exposure and prediction of alcohol disorders in early adulthood: a birth cohort study. Arch. Gen. Psychiatry 63, 1009-1016. doi: 10.1001/archpsyc.63.9.1009

Baculis, B. C., Diaz, M. R., and Valenzuela, C. F. (2015). Third trimesterequivalent ethanol exposure increases anxiety-like behavior and glutamatergic transmission in the basolateral amygdala. Pharmacol. Biochem. Behav. 137, 78-85. doi: 10.1016/j.pbb.2015.08.009

Baer, J. S., Sampson, P. D., Barr, H. M., Connor, P. D., and Streissguth, A. P. (2003). A 21-year longitudinal analysis of the effects of prenatal alcohol exposure on young adult drinking. Arch. Gen. Psychiatry 60, 377-385. doi: 10.1001/archpsyc.60.4.377

Bakhireva, L. N., Garrison, L., Shrestha, S., Sharkis, J., Miranda, R., and Rogers, K. (2018). Challenges of diagnosing fetal alcohol spectrum disorders in foster and adopted children. Alcohol 67, 37-43. doi: 10.1016/j.alcohol.2017.05.004

Bliss, T. V., and Lomo, T. (1973). Long-lasting potentiation of synaptic transmission in the dentate area of the anaesthetized rabbit following stimulation of the perforant path. J. Physiol. 232, 331-356. doi: 10.1113/jphysiol.1973.sp010273

Boardman, J. D., Finch, B. K., Ellison, C. G., Williams, D. R., and Jackson, J. S. (2001). Neighborhood disadvantage, stress, and drug use among adults. J. Health Soc. Behav. 42, 151-165. doi: 10.2307/3090175

Cantacorps, L., Montagud-Romero, S., Luján, M. A., and Valverde, O. (2019). Prenatal and postnatal alcohol exposure increases vulnerability to cocaine addiction in adult mice. Br. J. Pharmacol. doi: 10.1111/bph.14901. [Epub ahead of print].

Carroll Chapman, S. L., and Wu, L. T. (2012). Substance abuse among individuals with intellectual disabilities. Res. Dev. Disabil. 33, 1147-1456. doi: $10.1016 /$ j.ridd.2012.02.009

Cobben, J. M., Krzyzewska, I. M., Venema, A., Mul, A. N., Polstra, A., Postma, A. V., et al. (2019). DNA methylation abundantly associates with fetal alcohol spectrum disorder and its subphenotypes. Epigenomics 11, 767-785. doi: 10.2217/epi-2018-0221

Cohen, E. J., Quarta, E., Bravi, R., Granato, A., and Minciacchi, D. (2017). Neural plasticity and network remodeling: from concepts to pathology. Neuroscience 344, 326-345. doi: 10.1016/j.neuroscience.2016.12.048

Flak, A. L., Su, S., Bertrand, J., Denny, C. H., Kesmodel, U. S., and Cogswell, M. E. (2014). The association of mild, moderate, and binge prenatal alcohol exposure and child neuropsychological outcomes: a meta-analysis. Alcohol. Clin. Exp. Res. 38, 214-226. doi: 10.1111/acer.12214

Gautam, P., Warner, T. D., Kan, E. C., and Sowell, E. R. (2015). Executive function and cortical thickness in youths prenatally exposed to cocaine, alcohol and tobacco. Dev. Cogn. Neurosci. 16, 155-165. doi: 10.1016/j.den.2015.01.010

Granato, A., and Dering, B. (2018). Alcohol and the developing brain: Why neurons die and how survivors change. Int. J. Mol. Sci. 19:E2992. doi: 10.3390/ijms19102992

Granato, A., Palmer, L. M., De Giorgio, A., Tavian, D., and Larkum, M. E. (2012). Early exposure to alcohol leads to permanent impairment of dendritic excitability in neocortical pyramidal neurons. J. Neurosci. 32, 1377-1382. doi: 10.1523/JNEUROSCI.5520-11.2012

Greenmyer, J. R., Klug, M. G., Kambeitz, C., Popova, S., and Burd, L. (2018). A multicountry updated assessment of the economic impact of fetal alcohol spectrum disorder: costs for children and adults. J. Addict. Med. 12, 466-473. doi: 10.1097/ADM.0000000000000438

Hatfield, D. (1985). Is social drinking during pregnancy harmless? Adv. Alcohol Subst. Abuse 5, 221-226 doi: 10.1300/J251v05n01_15

Hausknecht, K., Haj-Dahmane, S., Shen, Y. L., Vezina, P., Dlugos, C., and Shen, R. Y. (2015). Excitatory synaptic function and plasticity is persistently altered in ventral tegmental area dopamine neurons after prenatal ethanol exposure. Neuropsychopharmacology 40, 893-905. doi: 10.1038/npp.2014.265

Hellemans, K. G., Sliwowska, J. H., Verma, P., and Weinberg, J. (2010). Prenatal alcohol exposure: fetal programming and later life vulnerability to stress, depression and anxiety disorders. Neurosci. Biobehav. Rev. 34, 791-807. doi: 10.1016/j.neubiorev.2009.06.004

Hendriks, H., Van den Putte, B., Gebhardt, W. A., and Moreno, M. A. (2018). Social drinking on social media: content analysis of the social aspects of alcohol-related posts on Facebook and Instagram. J. Med. Internet Res. 20:e226. doi: 10.2196/jmir.9355

Johnson, J. L., and Leff, M. (1999). Children of substance abusers: overview of research findings. Pediatrics 103(5 Pt 2), 1085-1099.

Kauer, J. A., and Malenka, R. C. (2007). Synaptic plasticity and addiction. Nat. Rev. Neurosci. 8, 844-858. doi: 10.1038/nrn2234

Lovinger, D. M., White, G., and Weight, F. F. (1990). NMDA receptormediated synaptic excitation selectively inhibited by ethanol in hippocampal slice from adult rat. J. Neurosci. 10, 1372-1379. doi: 10.1523/JNEUROSCI.10-04-01372.1990

Mead, E. A., and Sarkar, D. K. (2014). Fetal alcohol spectrum disorders and their transmission through genetic and epigenetic mechanisms. Front. Genet. 5:154. doi: 10.3389/fgene.2014.00154

Milani, L., La Torre, G., Fiore, M., Grumi, S., Gentile, D. A., Ferrante, M., et al. (2018). Internet gaming addiction in adolescence: risk factors and maladjustment correlates. Int. J. Ment. Health Addict. 16, 888-904. doi: 10.1007/s11469-017-9750-2

Morini, L., Marchei, E., Tarani, L., Trivelli, M., Rapisardi, G., Elicio, M. R., et al. (2013). Testing ethylglucuronide in maternal hair and nails for the assessment of fetal exposure to alcohol: comparison with meconium testing. Ther. Drug Monit. 35, 402-407. doi: 10.1097/FTD.0b013e318283f719

Newton, N. C., and Lee, N. (2019). Evidence-based prevention and the need for tough decisions: Alcohol and other drug education in schools. Drug Alcohol Rev. 38, 595-596. doi: 10.1111/dar.12986

Olney, J. W. (2014). Focus on apoptosis to decipher how alcohol and many other drugs disrupt brain development. Front. Pediatr. 2:81. doi: 10.3389/fped.2014.00081

Olson, H. C., Streissguth, A. P., Sampson, P. D., Barr, H. M., Bookstein, F. L., and Thiede, K. (1997). Association of prenatal alcohol exposure with behavioral and learning problems in early adolescence. J. Am. Acad. Child Adolesc. Psychiatry 36, 1187-1194. doi: 10.1097/00004583-199709000-00010

Rivkin, M. J., Davis, P. E., Lemaster, J. L., Cabral, H. J., Warfield, S. K., Mulkern, R. V., et al. (2008). Volumetric MRI study of brain in children with intrauterine exposure to cocaine, alcohol, tobacco, and marijuana. Pediatrics 121, 741-750. doi: 10.1542/peds.2007-1399

Ross, E. J., Graham, D. L., Money, K. M., and Stanwood, G. D. (2015). Developmental consequences of fetal exposure to drugs: what we know and what we still must learn. Neuropsychopharmacology 40, 61-87. doi: 10.1038/npp.2014.147

Sanchez, J. J., Noor, S., Davies, S., Savage, D., and Milligan, E. (2017). Prenatal alcohol exposure is a risk factor for adult neuropathic pain via aberrant neuroimmune function. J. Neuroinflammation 14:254 doi: 10.1186/s12974-017-1030-3

Sarkar, D. K. (2016). Male germline transmits fetal alcohol epigenetic marks for multiple generations: a review. Addict. Biol. 21, 23-34. doi: 10.1111/adb.12186

Sharp, B. M. (2017). Basolateral amygdala and stress-induced hyperexcitability affect motivated behaviors and addiction. Transl. Psychiatry 7:e1194. doi: $10.1038 /$ tp.2017.161

Spear, N. E., and Molina, J. C. (2005). Fetal or infantile exposure to ethanol promotes ethanol ingestion in adolescence and adulthood: a theoretical review. Alcohol. Clin. Exp. Res. 29, 909-229. doi: 10.1097/01.ALC.0000171046.78 556.66

Vagnarelli, F., Palmi, I., García-Algar, O., Falcon, M., Memo, L., Tarani, L., et al. (2011). A survey of Italian and Spanish neonatologists and paediatricians regarding awareness of the diagnosis of FAS and FASD and maternal ethanol use during pregnancy. BMC Pediatr. 11:51. doi: 10.1186/1471-243111-51

Valenzuela, C. F., Morton, R. A., Diaz, M. R., and Topper, L. (2012). Does moderate drinking harm the fetal brain? Insights from animal models. Trends Neurosci. 35, 284-292. doi: 10.1016/j.tins.2012.01.006 
Wang, R., Shen, Y. L., Hausknecht, K. A., Chang, L., Haj-Dahmane, S., Vezina, P., et al. (2019). Prenatal ethanol exposure increases risk of psychostimulant addiction. Behav. Brain Res. 356, 51-61. doi: 10.1016/j.bbr.2018.07.030

Weiner, J. L., and Valenzuela, C. F. (2006). Ethanol modulation of GABAergic transmission: the view from the slice. Pharmacol. Ther. 111, 533-554. doi: 10.1016/j.pharmthera.2005.11.002

Yates, W. R., Cadoret, R. J., Troughton, E. P., Stewart, M., and Giunta, T. S. (1998). Effect of fetal alcohol exposure on adult symptoms of nicotine, alcohol, and drug dependence. Alcohol. Clin. Exp. Res. 22, 914-920. doi: 10.1111/j.1530-0277.1998.tb03889.x
Conflict of Interest: The author declares that the research was conducted in the absence of any commercial or financial relationships that could be construed as a potential conflict of interest.

Copyright (C) 2020 Granato. This is an open-access article distributed under the terms of the Creative Commons Attribution License (CC BY). The use, distribution or reproduction in other forums is permitted, provided the original author $(s)$ and the copyright owner(s) are credited and that the original publication in this journal is cited, in accordance with accepted academic practice. No use, distribution or reproduction is permitted which does not comply with these terms. 


\section{OPEN ACCESS}

Edited by:

Federico Manzi,

Catholic University of the Sacred

Heart, Italy

Reviewed by:

Daniela Bulgarelli,

University of Turin, Italy

Eleonora Cannoni,

Sapienza University of Rome, Italy

*Correspondence:

Giuliana Pinto

pinto@unifi.it

Specialty section:

This article was submitted to Developmental Psychology,

a section of the journal

Frontiers in Psychology

Received: 26 November 2019

Accepted: 27 January 2020

Published: 14 February 2020

Citation:

Pinto G, Cameron CA and Toselli M (2020) An Old Mechanism, Imitation, Geared for Socio-Material

Knowing in a "Day in the Life" of First Graders. Front. Psychol. 11:177. doi: 10.3389/fpsyg.2020.00177

\section{An Old Mechanism, Imitation, Geared for Socio-Material Knowing in a "Day in the Life" of First Graders}

\author{
Giuliana Pinto $^{1 *}$, Catherine Ann Cameron ${ }^{2}$ and Monica Toselli ${ }^{1}$ \\ ${ }^{1}$ University of Florence, Florence, Italy, ${ }^{2}$ University of British Columbia, Vancouver, BC, Canada
}

This paper adopts sociomateriality as a theoretical lens to further our understanding of how imitation acts to support the use of objects, and in doing so, constitutes a sociomaterial practice. Within a sociomaterial perspective we aimed to perform the analysis of imitation as a powerful way to learn how to use objects embedded into the practices within which the objects are constituted. The contribution of this approach is illustrated using the findings of the application of the quasi-ecological Day in the Life (DITL) methodology to the everyday lives of two 6-year-old children. Within a casestudy frame, we traced the children's imitation behaviors focused on the use of objects during an entire day of their life, the various people and practices with which they were associated, the multiple sociomaterial configurations that the objects assume, and the social and material consequences of their use. Imitation appears to be is a complex activity, involving multiple stakeholders who interact in order to facilitate the understanding of various artifacts across diverse knowledge domains, and enhance their interpretive flexibility across communities of practice.

Keywords: sociomateriality, learning, imitation, artifacts, children's daily life

\section{INTRODUCTION}

The meaning of socio-materiality that we adhere to in our research pertains to the way by which a culture encourages its members to interact with material objects. From using forks to eat, to maneuvering high-tech gadgets of modern times, humans are adept in swiftly learning to use a wide range of tools in their daily lives. Mastery of "tool use" implies a progression from learning to act "on" objects to learning to act "with" objects. Among the multiple resources that aid the learning process, the most important are social interactions. How particular modes of interaction are socialized, acquired and internalized by children is worthy of study.

We believe that the use of an object, from a most normative, to a more atypical, personally and socially manipulated useage is transmitted through imitation. Imitation is a powerful mechanism that stimulates the use of a material object, even if it is not the only one, and there are obvious affordances of an object itself. Imitation is a traditional learning and communication tool, long ago identified from (Thorndike, 1898, p. 50) as: "doing an act by seeing it done by someone else," but much debate has subsequently addressed and investigated this mechanism.

In this research we offer an operational definition, along with introducing certain theoretical concepts that represent imitative behavior. Moreover, different mechanisms underlying imitative behaviors and their different functions in use of material objects can readily be identified. Extensive research (following Meltzoff and Moore, 1977; Gopnik et al., 2000) has addressed 
infants' early imitation of motor imitation of adults in controlled experimental settings. Alternatively, an ecological approach to document and explore spontaneous imitative behaviors when objects are involved can be used to illustrate how sociomateriality is shared in childhood by imitation. Transmission of the use of an object corresponds to the transmission of its meaning, value and relational function. The interaction of this learning and communication can trigger the social use of an object.

From early pretend play onward, when a 2 -year-old infant "turns objects into symbols", as Rakoczy et al. (2005) assume, objects acquire a metarepresentational role. "The development of understanding symbolic actions with objects, we claim, is best considered as part of children's developing social understanding more generally, and the development of performing symbolic actions with objects is most fruitfully viewed as a process of cultural learning, based on children's nascent understanding of intentional action and on cultural scaffolding" (p. 69).

This cultural learning goes on in every form of interaction with objects performed in front of someone, and can transmit the practical as well as symbolical meaning of objects.

The concept of guided participation described by Rogoff (2003) offers instances where the interchange of the meaning of actions, objects, tools are obtained by imitation. The adult partner displays the use of an object in front of a child, thus demonstrating its meaning.

\section{THE METHODOLOGICAL CHALLENGE: THE "DAY IN THE LIFE" (DITL) LENS}

An examination of the phenomena involved in the induction of socio-materiality via imitation requires methodological choices: First of all, it is necessary to capture as intricately as possible the sequence of the model actions and those of replication: only in their sequence in time, in fact, does their imitative nature make sense and sanction their interpretation. It is then necessary to be able to place such actions in the context in which they take place, accessing a picture as rich as possible in detail, in terms of setting, rules and rhythms, other actors and characters. In this effort we have applied an innovative quasiecological methodology, the DITL method, originally developed by Gillen and Cameron (2010) and Gillen et al. (2007), whereby the investigators audio-visually record to be able afterward to observe, document, and explore the everyday transactions of young children in situ during one specific day which has been over the years confirmed to have both ecological and heuristic validity.

The original methodology has been applied to developmentally different aspects, allowing the researcher to deepen, in socio-cultural perspectives how children comprehend and co-construct (for instance) their symbolic and literacy abilities (Pinto et al., 2008, 2011, 2015), their affective experiences in the use of domestic spaces and their meanings of cultural values for instance (Cameron et al., 2014b; Gillen and Cameron, 2017).
To apply the DITL methodology to questions regarding children in the transition between exclusive home- to include formal schooling (Marsico et al., 2013), DITL researchers are obliged to adapt the methodology to accommodate the increase of ecological niches across which the children and their families transit through as they enter the school door (Bronfenbrenner, 1986; Sameroff, 2010). This involved the engagement of school authorities, of educational administrators and teachers, of community members including dance, art, swimming and music teachers, youth leaders and neighbors (Cameron and Hunt, 2018). As a result we have had access by audio-visually recording of the constant flow of actions and interactions that accompany and mark the passing of a child's day from his awakening to his falling asleep at night, through the various contexts (family, school) and with the various partners from time to time, from family, teachers, friends, and neighbors.

Such an approach as that involving the qualitative methods of the Day in the Life (DITL) procedures provides an efficacious avenue for this sort of dynamic, multifocal, culturally sensitive discovery (cf. Cameron and Pinto, 2020). Moreover following children for just 1 day during their first year of schooling, at home and at school shows the interaction, the zones of overlapping, both contributing in different but sometimes also common pathways to a child's knowing in every domain, from the strictly social, to the material. Liminality of education, the importance of border zones (Valsiner, 2013), starting formal schooling is a crucial event for young children and their families. How well children negotiate this transition is important, as it affects their long-term academic outcomes (Dockett et al., 2010); this process can be thought of in ecological terms: As children move from home to other learning environments, these environments become increasingly important to their development and the intersection of family and school is a crucial third locus for development and education (Sameroff, 2010).

\section{IMITATION IN A DITL OF CHILDREN IN TRANSITION TO SCHOOL}

Ecological observation of the everyday events of typically developing children, potentiated by a audiovisual methodology offers us a uniquely valuable lens through which to capture the interesting and underestimated form of early social learning: imitation. As many sociologists (cf. Berger and Luckman, 1967) presume, the meaning of life is concentrated in the experience of daily life events. Let us therefore define the meaning we attribute to imitation, as it is a concept worthy of careful consideration. Paulus (2011) stressed that a behavioral definition of imitation can be helpful. While broadly encompassing, this definition can also be wholly sharable.

"... imitation is designated in all the instances when infants show the same behavior a model has performed in front of them and as a consequence of the particular action the model has performed (and not as a consequence of any other behavior). We can clearly see that, assuming this analysis, the word imitation comes with some assumptions about the imitative behavior. Imitation is then used when a relation between two 
behaviors is assumed in which a second behavior is sufficiently similar and causally connected to a first behavior without concretely specifying the mechanism which subserved it in detail" (Paulus, 2011, p. 850).

Consequently:

“...when observing infants in daily life, we hardly ever know by which mechanism their imitative behavior actually was subserved, especially when we are aware that this might also change from situation to situation so that the same behavior in one situation could be caused by reading the intention of others and in another situation by emulation or mimicry. We only can state at a behavioral level that infants imitated somebody" (Paulus, 2011, p. 852).

Inasmuch as it would be possible to consider in imitative behavior every enactment that somehow reproduces an earlier one, including when the immediate presentation by the model is not in sight, delayed imitation might be considered. But considering examples of extended delays in imitation would also divert our efforts to identify imitation solely by visual support what was captured by in the audio-visual record. Thus, we do not consider in our research delayed imitation behavior, as they could misdirect our aims.

Having focused our attention on motor repetition to identify imitation, we have subscribed to Byrne's assumption that "imitation is magical" (Byrne, 2005, p. 225), as imitative behaviors by definition are just those behaviors that are not causally explained by the more common mechanisms of reinforcement. Accordingly, we hypothesize that this mechanism works very frequently in children's everyday lives (Toselli et al., 2018) and we will explore partners and settings that enhance using material objects in diverse ways, by imitation.

\section{METHODOLOGY}

Two Italian children were video-recorded during an entire DITL (cf., Toselli et al., 2018) each for ten-hour-long continuous episodes, across home (about $5 \mathrm{~h}$ ) and school engagement. During a DITL, a child is carefully filmed from the time s/he repetition. In advance of the filming, the primary socializers (in the home: the parents; in the school: the teachers) are interviewed and they collaborate on providing access to a usual Day's events, interactions and contexts of participation. The measures to reduce the interference constituted by the presence of the observer, consisted not only in the specific training and experience of the observer to that particular observational method, but also in the preliminary knowledge and presentation of the observers to the family in the days preceding the one chosen for the registration of the overall day. While the child is filmed, a note-taker records and maps contextual and cultural information, important in the interpretation the transactions viewed on film. No assumption of typicality in this cultural project is made. Our analysis simply draws upon this recorded corpus of naturalistic interactions between our participants, their adult interlocutors and their siblings and peers, selecting those transactions involving imitation of interactions with material objects. Our two participating first graders were observed while they successfully navigated 1 day during their first year in primary school, following their preschool experiences. They were identified as healthy and thriving children in the framework of a positive psychological research initiative. The videotaped material inspired this investigation of motor imitation pertaining to the use of objects, observable by the careful inspection of the visual data recorded by the videotapes. We therefore identified every motor sequence that was repeated or inspired by each of the two participants under study. On the corpus of video recordings relating to the entire day of the two participants, two independent judges, members of the research team, carried out a visual examination of the material with the task of detecting all the episodes in which motor imitation behavior as previously defined was present, creating a list. The comparison between the choices made by the two judges revealed a 100\% agreement. The episodes reported are therefore all those identified with the agreement of the two judges. The interpretation of the events thus identified was carried out jointly by the authors, and elaborated in the form of a discussion before articulating the commentary of each of the episodes reported below, with a cyclically inductive and deductive approach, from data to theory and vice versa. The selected imitation situations were extracted from the stream of the daily interactions, by two observers, independently inspecting the $10 \mathrm{~h}$ of video-recordings of each child's "Day." One videographer followed and filmed each child throughout their day while a second researcher took careful contextual notes. There was minimal verbal communication between the researchers and participants during the recording of the $\operatorname{day}(s)$. Rather, semi-structured interviews were conducted before and after the data collection day. Before hand, participants (child, parents, siblings, teachers, and peers) were fully appraised in advance as to the procedures they were to expect, and afterward, participants, their families and teachers were shown a half-hour compilation of filmed clips of the full filmed day and asked to comment in general on the selected clips and specifically, as to whether they were somewhat representative of the child's daily life.

\section{PARTICIPANTS}

Our two Italian first graders are a boy, 6,7 year-old Martino, and Sara, a 7,1 year-old girl, both attended the same class in a school in a suburban area on the outskirts of a city in Central Italy, characterized by a medium socio-economic level. In Italy, most children (approximately 99\%) are enrolled in state schools, which thus provides a representative cross section of the Italian population among kindergartners and primary school students. the Italian population is characterized by a very low mobility, and children tend to attend schools in the same neighborhood.

The research was performed in the first year of a State primary school, attended by children between 6 and 7 years of age. The aim of this level, compulsory, in the education system is to provide pupils with basic learning and the basic tools of active citizenship. Primary education is divided, for teaching purposes only, into a first year, linked to pre-primary school, followed by a further two levels of 2 years each. In primary schools, children, according to their age, are organized into groups called "classes." The class where we worked with 22 pupils and, adopting the 
weekly school timetable of $30 \mathrm{~h}$, had only one teacher, a generalist supported by an English language teacher. The standard school day consists of a total of $7 \mathrm{~h}$, during which from 8.30 a.m. to 1 p.m. and from 2.30 p.m. to 4.30 p.m. the teaching activity takes place. While from 1 p.m. to 2.30 p.m. the meal is eaten inside the school structure in the presence of special staff.

Children in the Italian educational system typically start kindergarten at age three and finish when they are five. Then, children enroll in primary school when they are 6 years old. Moreover, in Italian schools, children are exposed to formal reading as soon as primary school begins. By contrast, the national curriculum for kindergarten does not include the formal teaching of reading and writing. According to national guidelines (Law n. 254 of November 16, 2012), first-grade children are expected to learn the instrumental level of the written language (reading and spelling) and the basics of mathematics (arithmetic, logic, geometry, and measuring). Teachers-students ratios are 2:28 in kindergartner and 1:25 in primary school.

The two participants, Sara and Martino, also share cultural environments in terms of the resources available to their families, and the character of the communities in which children live, including the economic climate and accessibility of appropriate services. The local community whose population is $95 \%$ Italian, is predominately middle-class, with most parents having had at least some college education.

Sara is attending the first year of a public Italian primary school, and she and her older brother live with their parents, who are both professionally employed. The family lives in a detached home, within walking distance of the school. Martino, also lives with his parents, both professionals, and his younger sister in an apartment with a garden in a renovated farmhouse. He also goes to school on foot, generally accompanied by his father.

As part of our ethical procedures, school authorities and parents offered informed consent for participation in the study and the children afforded active assent.

\section{DATA ANALYSIS}

All instances during which partners show the same motor behavior (addressing an object) that a model has performed in front of them, that is, doing an act after seeing it done were identified. The participants can be either the copier or the model, as it is common in this age old. As a note of caution, a single situational description cannot provide conclusive evidence to the reader of what happened in the dynamic flow of the video recording. Thus the instances were interpreted to identify the various forms of cultural learning that took shape when children, at the beginning of primary school are ubiquitously imitating the use of culturally significant objects. Our expectation is that imitation can lead to the learning of different modalities and nuances about the functions and rules with which objects can be used (i.e., their pragmatics), depending on the model and context. Across diverse social environments, that differ in the extent and the conditions with which children are engaged by significant others.

\section{FINDINGS}

There were a total of 23 separate imitative episodes in the daylong video-recorded material pertaining to Sara and 30 imitations in the video-recoding of Martino's "Day."

Among these imitations, we identified some 8 of them that involved the use of objects: 3 for Sara and 5 for Martino.

We first present the 3 imitations where a normative use of objects is demonstrated by the model and subsequently executed by the imitating partner and then, we describe the 5 more original and subjectively laden imitative use of objects. Some imitative scripts involve adults as well as peers. Episodes occurred at home as well as at school.

(1) The most typical episode where a normative use of objects is shown involved an adult. Sara is engaged in a craft activity with her mother at home, creating a bracelet with glass beads: Mother shows her how to craft the bracelet. Her mother offers a model for enacting a behavior that is to be acquired by her child. Sara engages in an imitative activity during a session of what Rogoff (2003) would refer to as guided participation. The girl waits to see her mother's pearl threading and immediately imitates the simple procedure, then proceeds by herself, replicating the newly learned behavior. While proceeding with her work she asks her mother when to stop stringing a strand of beads, the mother shows her how to measure, around the child's arm, the necessary length of the bracelet, and Sara, after adding some beads, uses the same measuring procedure.

(2) In peer tutoring, however, reciprocity emerges. After school, at home, Martino and a schoolmate, who accompanied Martino home from school, are playing football. One of them knows how to advance the ball with his head, the other, with his foot. They reciprocally enact for one another the motor performance and model for each other. Reciprocity is a hallmark of imitation between peers, and less frequently, in exchanges with adults, who most characteristically only deploy reciprocity in early interactions with very young children. Playing football represents the social and cultural contexts in which these children live: Socio-cultural variations depend not only upon the attitudes of parents, teachers, and society in general, but also on such variables as the amount of play space and time that is available to children Martino and his schoolmates help each other in an imitative activity that produces some physical acts to pursue specific goals according to codified rules.

(3) Older brothers are also typical models for the canonical use of objects by younger siblings. During breakfast, while still at home, Martino's little sister, even if without the specific need to wipe her mouth, as soon as Martino has wiped his mouth, she immediately imitates him, she too uses a paper towel that is on the breakfast table. Table manners evolve within cultures, varying widely between different cultures and countries, and they exert a useful function in intercultural adaptation. To master fundamental knowledge of table manners in a culture enhances intercultural communication awareness and intercultural adaptation. In their mutability from one culture to another, they constitute a good example of an "opaque rule," that is, highly conventional, arbitrary and unpredictable. To learn 
good manners at the table, imitation appears to be a powerful, if not the only, aid.

The use of objects can also present indications of creativity, introducing varied interactions with them, which can also convoy a personal communicative message about the object's meaning of its use in a relationship.

(1) A creative, playful activity can arise: When Sara plays with her shoe laces, as if they were musical instrument strings, two schoolmates discover this new behavior and subsequently play with their own shoe laces as strings, demonstrating a kind of a diversified imitation. Learning requires an interactive balance of gaining the facts and skills required by the culture and making information one's own. We are shown, in this imitative istance, how children enact this interactive cycle, that helps them to understand the use of objects in an intrinsically motivating way.

(2) Still at school, during the common meal in the canteen, we detected an immediate and exact imitation by Martino, observing and then replicating a schoolmate's dunking a cracker in his glass of water before eating it. This is an original use in our food culture, where crackers are not softened in cold water, but rather in other, usually hot, beverages! Interaction with peers in less rigid situations than academic routines, such as those allowed during school meals shared together, offers valuable opportunities to children to act and behave like people they know. Freedom to use "traditional" objects encourages creative expression of ideas and understandings.

(3) Again at school, during the school meal, we have an imitation of a procedure for managing the precarious positioning of a full water pitcher, on the edge of the dining table, beside a glass, also full of water. This behavior is reenacted by a schoolmate who follows Martino and asks him for explicit instructions and demonstrations in order to get the same result as the model. This more perilous activity, which could produce a mess on the table, clearly shows the atypical use of objects, introducing in it a prohibited aspect, if noticed by the teachers. Martino and his companions show that they know how to use hybrid spaces to navigate between rules and invention, experimenting with the borderline boundaries of the conventional use of objects.

(4) During a recreation period at school, at a time that is not typically devoted to instructional learning, such as during formal lessons, we observed a more aggressive kind of imitative behavior, involving a materially valued object. A group of children are looking at a sticker album placed on the ground out in the garden. Martino steps onto the album with shoed feet, interrupting his schoolmates' activity. One of his admirers immediately steps in the same way onto the album. Culturally representational materials help children understand the social and ethnic values of their communities. Such social contexts provide them with an arena for refining their social skills also through the conception and imitation of transgressive behaviors, in which the cultural value of objects is challenged.

(5) At home Sara is playing in the room she shares with her older brother. The atmosphere is playful and somehow conflictful because the two children are debating about who is the owner of various plush animal toys that are in a basket. Sara begins throwing the toys in the air and also at her brother. The brother immediately recognizes the provocative value of this activity and does the same with the objects, especially those that he believes to be property of his sister. In this interaction, children exploit the opportunity to move freely from one area to another and to engage in recreational activities in spaces of the house that are not continuously and directly under the control of adults. Imitationbased learning activities provide multiple ways for children to learn to use complex, challenging, and varied materials.

\section{DISCUSSION}

This paper takes up the socio-material perspectives as an avenue for understanding the role of imitation in learning to use objects in family and school practices, assuming that the range between informal and formal learning can be conceptualized as a continuum.

Our visual theoretical approach and its consequential methodologies deployed within the DITL sociocultural framework proved to be effective, deepening our understanding of collaborative construction of the use of objects during a critical life transition namely, when they first gain significant learning experiences outside the home, and specifically in a formal school setting (Cameron et al., 2014a). Using examples of children's acts of imitation during their filmed Day, we documented how school and home are contexts populated by significant others whose knowledge and practices offer to the child multiple models learning true imitation. We also discovered how frequent, multifaceted and powerful imitation exists across contexts and partners and how children use imitation in a collaborative and communicative manner as a powerful meaning-making tool. The procedure adopted in the DITL research allowed us to provide rich information regarding the thinking, talking, and social interaction that naturally occurred "around" imitations the child perform across the continuities and discontinuities that characterize the way in which objects are used in the various systemic practices.

We discovered from our observations that imitation in the motor use of objects resides in the commonplace of children's every day lives. These uses transmit a normative and sometimes a more creative, subjectively and emotionally laden use of objects.

In this double context of school and home we noticed that whether our recordings pertained school- or home-time, is was mostly when the children interacted freely, that the guided participation with the use of objects was enacted. Our participants demonstrated both agency and communitarianism in this period of their lives, just as we reported of our young Canadian participants (Dmytro et al., 2014), characterized by many new and often conflicting demands for competence. Formal schooling classically pertains to the immaterial, abstract world of learning and explicitly encourages, in a collaborative framework, imitation by pupils, aimed at academic tasks and hewing to the rules of the classroom culture. But, as far as we have been able to observe on the school day of Sara and Martino, it is not the objects and their utilities that are prompting imitation, but rather the practical navigation of school rules and the pragmatics of addressing assigned academic tasks. It is in free time that, at this age, in transactions with adults as well as peers, 
knowledge of the material world is tested, explored and acquired. The family, less focused on explicit teaching, more frequently uses demonstrations of what the child needs to imitate to use instruments, as was the case of Sara and her mother crafting the bracelet. Differences emerge also between partners: Adults can be imitated, but they seldom-to-never imitate children, while with peers reciprocity is more common, as in Martino's dangerous play with the pitcher full of water. We discover in these first graders' lives the wide role expressed as models by peers as to the material world. It is quite obvious that the amusing, trasgressive role of managing objects is particularly shared by peers!

Focusing on children's imitative behaviors we aimed further to enrich our understanding of the young child's perspectives. Adopting a visual methodology we had the opportunity not only listening to verbal communication, but also checking the child's different forms of expressing non-verbal emotions, meanings and sense of everyday events. When researchers attempt to capture the lived experiences and sense of everyday events from the young child's perspective through the use of visual methodologies, they acknowledge the unspoken voice of the child which is always present in their affective, active engagement with others and the environment (Quiones, 2014).

Social scientific research also does not yet reveal a deep enough understanding nor does it apply ready opportunities for exploring such mechanisms as imitation that we share with so many species and, living beings, beyond childhood and across the life span. This paper has argued strongly for legitimizing imitation as an appropriate learning tool in schools and other educational settings. We tend to be blind to the allpervasive constructivist imitative nature of human beings and particularly young children, and unaware of its implications for

\section{REFERENCES}

Berger, B. G., and Luckman, T. (1967). The Social Construction of Reality. Harmondswoth: Penguin.

Bronfenbrenner, U. (1986). Ecology of the family as a context for human development: research perspectives. Dev. Psychol. 22, 723-742. doi: 10.1037/ 0012-1649.22.6.723

Byrne, R. W. (2005). "Detecting, understanding, and explaining imitation by animals," in Perspectives on Imitation, eds S. Hurley, and N. Chater (Cambridge, MA: MIT Press), 225-242.

Cameron, C. A., and Hunt, A. K. (2018). "A Day in the Life": A Visual, Multimedia Approach to Research. London: Sage Research Methods Cases, doi: 10.4135/ 9781526449863

Cameron, C. A., Hunt, A. K., Pezzica, S., Phillips, L., Heydon, R., and Kumpulienen, K. (2014a). Children shaping their identities as readers and writers: a Day in the Life of children in transition to school. Child. Explorer 1, $22-25$.

Cameron, C. A., Pinto, G., and Tapanya, S. (2014b). Scaffolding one Thai youth's drawing toward resilience. Cult. Psychol. 20, 453-476. doi: 10.1177/ $1354067 \times 14551293$

Cameron, C. A., and Pinto, G. (2020). Literacies as contexta: notational acts during a day in the life. Interchange (in press).

Dmytro, D., Kubiliene, N., and Cameron, C. A. (2014). Agentive and communitarian play in early childhood. Early Child Dev. Care 184, 1920-1933. doi: 10.1080/03004430.2014.894033

Dockett, S., Perry, R., and Kearney, E. (2010). School Readiness: What Does it Mean for Indigenous Children, Families, Schools and Communities? Closing the Gap Clearinghouse. Canberra: Australian Institute of Health and Welfare. parenting and educating. In their everyday lives, children are busy observing those around them and interpreting the world accordingly: Imitation plays a crucial role in the transmission and maintainance of relevant cultural knowledge, specially suited for the demand characteristics of cultural forms (as the symbolic tools and instruments), whose causal, functional, or intentional nature is cognitively opaque to the learner (Gergely and Csibra, 2006) who can therefore only acquire them through imitative actions.

\section{DATA AVAILABILITY STATEMENT}

The datasets generated for this study are available on request to the corresponding author.

\section{ETHICS STATEMENT}

The study involved human participants and was approved by the Ethical Review Committee of the Department of Psychology, University of Florence. School authorities offered informed consent, parents provided written informed consent for the children's participation, and the child-participants afforded active assent.

\section{AUTHOR CONTRIBUTIONS}

All authors listed have made a substantial, direct and intellectual contribution to the work, and approved it for publication.

Gergely, G., and Csibra, G. (2006). "Sylvia's recipe: the role of imitation and pedagogy in the transmission of cultural knowledge," in Roots of Human Sociality: Culture, Cognition, and Human Interaction, eds N. J. Enfield, and S. C. Levenson (Oxford: Berg Publishers), 229-255.

Gillen, J., and Cameron, C. A. (2010). International Perspectives on Early Childhood Research: A Day in the Life. Houndmills: Palgrave Macmillan.

Gillen, J., and Cameron, C. A. (2017). Negotiating citizenship: a young child's collaborative meaning-making constructions of beavers as a symbol of Canada. Lang. Educ. 31, 330-350. doi: 10.1080/09500782.2017.1302466

Gillen, J., Cameron, C. A., Tapanya, S., Pinto, G., Hancock, R., Young, S., et al. (2007). 'A day in the life': advancing a methodology for the cultural study of development and learning in early childhood. Early Child Dev. Care 177, 207-218. doi: 10.1080/03004430500393763

Gopnik, A., Meltzoff, A. N., and Kuhl, P. K. (2000). The Scientist in the Crib: What Early Learning Tells us About the Mind. New York, NY: HarperCollins.

Marsico, G., Komatsu, K., and Iannaccone, A. (eds) (2013). Crossing Boundaries. Intercontextual Dynamics Between Family and School. Charlotte: Information Age Publishing.

Meltzoff, A. N., and Moore, M. K. (1977). Imitation of facial and manual gestures by human neonates. Science 198, 75-78. doi: 10.1126/science.198.4312.75

Paulus, M. (2011). Imitation in infancy: conceptual considerations. Theor. Psychol. 21, 849-856. doi: 10.1177/0959354310395990

Pinto, G., Accorti Gamannossi, B., and Cameron, C. A. (2008). “Joint book reading: socialization of literacy in cultural perspective," in Yearbook of Idiographic Science, Vol. 1, eds S. Salvatore, J. Valsiner, S. Strout-Yagodzynski, and J. Clegg (Roma: Firera \& Liuzzo Group), 287-306.

Pinto, G., Accorti Gamannossi, B., and Cameron, C. A. (2011). From scribbles to meanings: social interaction in different cultures and the emergence of young 
children's early drawing. Early Child Dev. Care 181, 425-444. doi: 10.1080/ 03004430903442001

Pinto, G., Cameron, A. C., Hunt, A., and Leger, P. (2015). Emerging literacy during one "Day in the Life" in the transition to school. Early Child Dev. Care 186, 1476-1490. doi: 10.1080/03004430.2015.1105800

Quiones, G. (2014). "A visual and tactile path: affective positioning of researcher using a cultural- historical visual methodology," in Visual Methodologies and Tools for Researching with Young Children, eds M. Fleer, and A. Ridgway (Netherlands: Springer).

Rakoczy, H., Tomasello, M., and Striano, T. (2005). "How children turn objects into symbols: a cultural learning account," in Symbol Use and Symbol Representation, ed. L. Namy (New York, NY: Erlbaum).

Rogoff, B. (2003). The Cultural Nature of Human Development. Oxford: Oxford University Press.

Sameroff, A. (2010). A unified theory of development: a dialectic integration of nature and nurture. Child Dev. 81, 6-22. doi: 10.1111/j.1467-8624.2009. 01378.x

Thorndike, E. L. (1898). Animal intelligence: an experimental study of the society processes in animals. Psychol. Monogr 2:50. doi: 10.1186/s13059-015-0623-3
Toselli, M., Pinto, G., and Cameron, C. A. (2018). “Aprender por meio da imitação no dia a dia em casa e na escola," in Psicologia do Desenvolvimento - "Um Dia na Vida" de Pessoas Bem-Sucedidas - Metodologia da Pesquisa Audiovisual em Psicologia Transcultural, Educação e Comunicação, eds C. Stella, and C. A. Cameron (Brazil: Jurua Editora - Psicolog Do Desenvolvimento), 91-108.

Valsiner, J. (2013). "Liminality of education. The importance of border zones," in Crossing Boundaries: Intercontextual Dynamics Between Family and School, eds G. Marsico, and K. K. Iannaccone (Charlotte: Information Age Publishing).

Conflict of Interest: The authors declare that the research was conducted in the absence of any commercial or financial relationships that could be construed as a potential conflict of interest.

Copyright (c) 2020 Pinto, Cameron and Toselli. This is an open-access article distributed under the terms of the Creative Commons Attribution License (CC BY). The use, distribution or reproduction in other forums is permitted, provided the original author(s) and the copyright owner(s) are credited and that the original publication in this journal is cited, in accordance with accepted academic practice. No use, distribution or reproduction is permitted which does not comply with these terms. 


\title{
Learning Process of Gaze Following: Computational Modeling Based on Reinforcement Learning
}

\author{
Mitsuhiko Ishikawa ${ }^{1,2 *}$, Atsushi Senju ${ }^{3}$ and Shoji Itakura ${ }^{1}$ \\ 'Department of Psychology, Graduate School of Letters, Kyoto University, Kyoto, Japan, ${ }^{2}$ Japan Society for the Promotion \\ of Science, Tokyo, Japan, ${ }^{3}$ Centre for Brain and Cognitive Development, Birkbeck, University of London, London, \\ United Kingdom
}

\section{OPEN ACCESS}

Edited by:

Antonio lannaccone,

Université de Neuchâtel, Switzerland

Reviewed by:

Elizabeth Ann Simpson,

University of Miami, United States

Shota Uono,

Organization for Promoting

Neurodevelopmental Disorder

Research, Japan

Guangyu Zeng,

University of Miami, United States, in

collaboration with reviewer EAS

${ }^{*}$ Correspondence:

Mitsuhiko Ishikawa

ishikawa.mitsuhiko.23r@

st.kyoto-u.ac.jp

Specialty section:

This article was submitted to Developmental Psychology, a section of the journal

Frontiers in Psychology

Received: 25 November 2019

Accepted: 30 January 2020

Published: 03 March 2020

Citation:

Ishikawa M, Senju A and Itakura S (2020) Learning Process of Gaze Following: Computational Modeling

Based on Reinforcement Learning.

Front. Psychol. 11:213.

doi: 10.3389/fpsyg.2020.00213
Many studies have explored factors which influence gaze-following behavior of young infants. However, the results of empirical studies were inconsistent, and the mechanism underlying the contextual modulation of gaze following remains unclear. In order to provide valuable insight into the mechanisms underlying gaze following, we conducted computational modeling using Q-learning algorithm and simulated the learning process of infant gaze following to suggest a feasible model. In Experiment 1, we simulated how communicative cues and infant internal states affect the learning process of gaze following. The simulation indicated that the model in which communicative cues enhance infant internal states is the most feasible to explain the infant learning process. In Experiment 2, we simulated how individual differences in motivation for communication affect the learning process. The results showed that low motivation for communication can delay the learning process and decrease the frequency of gaze following. These simulations suggest that communicative cues may enhance infants' internal states and promote the development of gaze following. Also, initial social motivation may affect the learning process of social behaviors in the long term.

Keywords: gaze following, reinforcement learning, computational modeling, infant internal state, communicative cues

\section{INTRODUCTION}

Human infants show face preferences from the very early stages of life (Johnson et al., 1991; Valenza et al., 1996). Especially, newborns have sensitivity toward human eyes (Farroni et al., 2002). Studies have found that 2- to 5-day-old newborns discriminated between direct and averted gaze, and they were faster to make saccades to peripheral targets cued by gaze direction (Farroni et al., 2004). These studies suggest that infants may have a rudimentary form of gaze following from immediately after birth.

Many studies have suggested from which age infants start gaze following. The earliest precursor of gaze following was observed from 3 months (D'Entremont et al., 1997). In general, it is said that infants show gaze following from 6 months (Butterworth and Jarrett, 1991; Gredebäck et al., 2010).

Previous studies also investigated the contexts that trigger infant gaze following, and the results from these studies informed theoretical perspectives. For example, infants follow others' gaze where accompanied by ostensive (communicative) cues (Senju and Csibra, 2008; Hernik and Broesch, 2019) or a highly attention-grabbing action (Szufnarowska et al., 2014). Ostensive 
cues or communicative cues were defined as signals showing communicative intent such as eye contact, while attentiongrabbing cues did not include communicative intent but with visually salient movement. Other studies failed to find such a contextual modulation (Gredebäck et al., 2018). In the following section, we briefly describe the factors that have been argued to affect gaze following in infants.

\section{Communicative Cues}

The theory of natural pedagogy suggests that infants follow others' gaze because they refer to the topic of communication within the framework of ostensive-referential communication (Csibra and Gergely, 2009). This theory predicts that infant gaze following should be most prominent when it follows ostensive cues such as direct gaze and infant-directed speech (Csibra, 2010). Ostensive cues can signal that a partner interacting with an infant has communicative intent transferring knowledge (Csibra and Gergely, 2009).

In the first empirical study on the effect of ostensive signals on gaze following, Senju and Csibra (2008) showed that 6.5month-old infants follow others' gaze when it followed eye contact or infant-directed speech (communicative cues), but not when it followed attention-grabbing stimuli (e.g., nonsocial animation overlaid on top of the actor's face). Based on this result, Csibra (2010) suggested that gaze following only occurs in the narrow context of ostensive cues early in life. Recently, Hernik and Broesch (2019) replicated this finding in 5- to 7-month-old infants developing in Vanuatu community, suggesting that the phenomenon is not fully dependent on a Western style of parenting. However, other studies showed conflicting evidence. For example, Szufnarowska et al. (2014) showed that 6-month-old infants followed others' gaze direction when it followed a highly attentiongrabbing, but not communicative, cue. In this study, total fixation duration to the model's head during her action, attention-getting phase, was compared as an index of infant attention, and it was lower in the no-cues condition than in each of the other attention-grabbing conditions. Moreover, a recent study from the same group (Gredebäck et al., 2018) found that gaze following in 6-month-old infants was not different between ostensive (eye contact), attention grabbing (shivering), and no cue (no head movement), suggesting that infants follow others' gaze without ostensive cues. The results suggest that infants' gaze-following behavior is not fully dependent on the presence of preceding ostensive signal in some context.

\section{Infant Attention}

Infant attention has been suggested as one of the factors affecting gaze following. As discussed above, Szufnarowska et al. (2014) showed that 6-month-old infants followed others' gaze direction which followed highly attention-grabbing cues such as shaking a head horizontally. From these results, it was suggested that gaze following is based on infant attention.

However, such a viewpoint contradicts Senju and Csibra (2008) which did not observe gaze following in the situation with an attention-grabbing animation on the model's face (see also Hernik and Broesch, 2019). In addition, as discussed above, Gredebäck et al. (2018) failed to show the effect of attentiongrabbing cue on gaze following. The results suggest that infant gaze-following behavior cannot be induced only by attentiongrabbing stimuli.

Many studies have investigated infant gaze following; however, the results were not consistent. For example, looking times to an actor's face were different in each study. Previous studies used looking time to the actor's face to measure infant attention. Szufnarowska et al. (2014) showed that eye contact attracted more infant attention than a no-cues condition. On the other hand, Gredebäck et al. (2018) indicated that infants looked at the actor in the shivering condition (social and non-ostensive cue) longer than in both the eyecontact condition and the no-cues condition. Although some studies suggested that infant attention affects gaze following, looking times to an actor's face do not always correlate with gaze following (Gredebäck et al., 2018; Ishikawa and Itakura, 2019), suggesting that looking time may not capture infant engagement in the task.

\section{Correlation Between Communicative Cues and Infant Internal State}

Ishikawa and Itakura (2019), by contrast, used heart rate as an alternative measurement of infants' internal states and suggested that (a) infants' gaze-following behavior is related to infants' physiological arousal and (b) looking time to the actor's face may not predict gaze following or infant internal state including attentional and physiological arousal measured by heart rate. In this study, there were three conditions, eye-contact, no-cues, and shivering conditions. The results of their study revealed that eye contact enhanced heart rate levels in 10-month-old infants, although there was no difference of looking time to the actor's face across conditions. Also, infants showed gaze following above chance level only with eye contact, consistent with a claim derived from the theory of natural pedagogy. Interestingly, infant heart rate levels during an actor's action predicted later gaze following in situations both with and without communicative cues and partially mediated between the conditions of communicative cues and gaze following. It has been suggested that physiological arousal is related to sensitivity and responsiveness to external stimuli (AstonJones et al., 1991, 1999). Infant studies have also shown results supporting the relation between physiological arousal and attentional state (Wass et al., 2016; de Barbaro et al., 2017). Also, empirical studies have shown that affective states and reward expectations can be reflected in physiological arousal (Critchley et al., 2005; Tummeltshammer et al., 2019). Because it is difficult to define which factors induce infant physiological arousal in gaze-following situations, here we use a broad concept of internal state, which could be measured by neurophysiological measurements.

Therefore, it can be considered that communicative cues affect the infant's internal state, which may be reflected in physiological arousal, and both communicative cues and the infant internal state may promote infant gaze following. 


\section{Importance of Computational Models}

Theories of the emergence of gaze following have been examined in behavioral experiments. However, the results of empirical studies were inconsistent, and the mechanism of gaze following remains unclear. This could partly be because it is difficult to include all factors related to gaze following and conduct many trials in infant behavioral experiments. Also, individual differences were difficult to assess with the small numbers of participants typically included in empirical infant studies. We conducted computational modeling of infant gaze following in an attempt to address these issues and complement empirical studies.

Computational modeling allows us to examine what is difficult to conduct with real infants in experimental settings, and it is very useful to theorize human development (Triesch et al., 2006). Triesch et al. (2007) used computational modeling to simulate the emergence of gaze following. Because reward-driven learning can be found from a very early developmental stage (Floccia et al., 1997) and suggested as a principal learning mechanism (Sutton, 1988), they applied reinforcement learning to modeling and suggested how gaze following emerges in the mother-infant interaction. However, their model did not include communicative cues, and their simulations were mainly based on the theory of mirror neuron system (Triesch et al., 2007).

It has been shown that communicative cues facilitate infant learning in the social context (Csibra and Gergely, 2009). Also, it has been suggested that looking at the same object with another person is rewarding for infants (Moore and Corkum, 1994; Mundy, 1995). Thus, in the learning process of gaze following, contextual information such as communicative cues may affect reinforcement. The modeling is not informative as to the conflicting results reported in recent empirical studies; more precisely, communicative cues and infant internal states were not taken into account in previous simulation studies (Triesch et al., 2006, 2007). Computational modeling with the factors examined in experimental settings may offer a new perspective on the mechanism of gaze following.

\section{The Purpose of This Study}

In this study, we simulated the learning process of infant gaze following and suggest a feasible model according to the results of empirical studies. It has been shown that reinforcement learning is the fundamental learning process in humans and is neurally plausible (Dayan et al., 2001; Holroyd and Coles, 2002); therefore, we applied reinforcement learning to simulate the early learning process of gaze following in infants and examined how infant internal states and communicative cues affect gaze following. Although computational modeling cannot compare models' feasibilities statistically, it is suggested that computational modeling may be particularly helpful to theorize because it can easily monitor all changes in the model (Triesch et al., 2006). To theorize about the development of gaze following, we compared three models, the communicative cue model, the communicative cue and infant internal state model, and the model in which communicative cues enhance infant internal state.

\section{METHODS}

\section{Environment and Parameters}

In the previous computational modeling of gaze following (Triesch et al., 2006, 2007), learning environment was posited that an infant and a caregiver interact with a number of objects, not only with a gaze target and a distractor. In a more complex way, they posit that caregiver's gaze direction was not always perfectly aligned with the caregiver's head orientation. Also, in the previous model, object locations were randomly distributed. Because they focused on creating a general model of gaze following interactions, and not on examining the effects of communicative cues and infant internal states, their simulation included the process of infant visual system affected by object saliency, caregiver's saliency, or infant visual field.

In order to examine the effects of communicative cues and infant internal states, we simplified experimental situations to be based on previous empirical studies (Senju and Csibra, 2008; Szufnarowska et al., 2014; Gredebäck et al., 2018; Hernik and Broesch, 2019; Ishikawa and Itakura, 2019; Figure 1), and actor's gaze direction was always consistent with the head orientation. An actor looked toward one of two objects with or without communicative cues. Two objects had the same saliency, and there was no looking bias. Infants were postulated to look to one of two objects $100 \%$ at the end of a trial. There were two considerable options of behavior for the infants, (a) following the actor's gaze or (b) looking toward one of the two objects randomly. The learning process was simulated by the Q-learning algorithm, which is one of the most popular reinforcement learning algorithms (Watkins and Dayan, 1992). The Q-learning algorithm is as follows in (1):

$$
Q_{[t+1]}=Q_{[t]}+\alpha \times\left(R \times P_{[r]}-Q_{[t]}\right)
$$

In Q-learning, a learned decision policy is determined by the behavioral value function, described as $Q$. To limit infant learning in a trial, learning rate " $\alpha$ " was the same in all simulations. Also, the reward probabilities $(\mathrm{Pr})$ were $100 \%$ for gaze following and $50 \%$ for random looking. This is because we posit that infants feel rewarded when they look at the same object as a model (Moore and Corkum, 1994; Triesch et al., 2006). The reward value $(R)$ was 1 in all the simulations. Each simulation was continued up to 2,000 trials. We compared how the behavioral values of gaze following were updated during 2,000 trials. We adopted a "softmax" strategy for selecting the infants' actions in all simulations. In the soft-max strategy, the worthiest action is still given the highest selection probability, but all the others are ranked and weighted according to their value estimates (Sutton and Barto, 1998). All the parameters used in the simulations are shown in Table 1.

\section{Communicative Cue Model}

With communicative cues, it is considered that infants can expect that an interacting partner is transferring knowledge (Csibra and Gergely, 2011). Therefore, we set up that communicative cues modulated the subjective reward probability. We added a variable named " $C$ " (communicative cue) into the QL formula. 


\section{A}

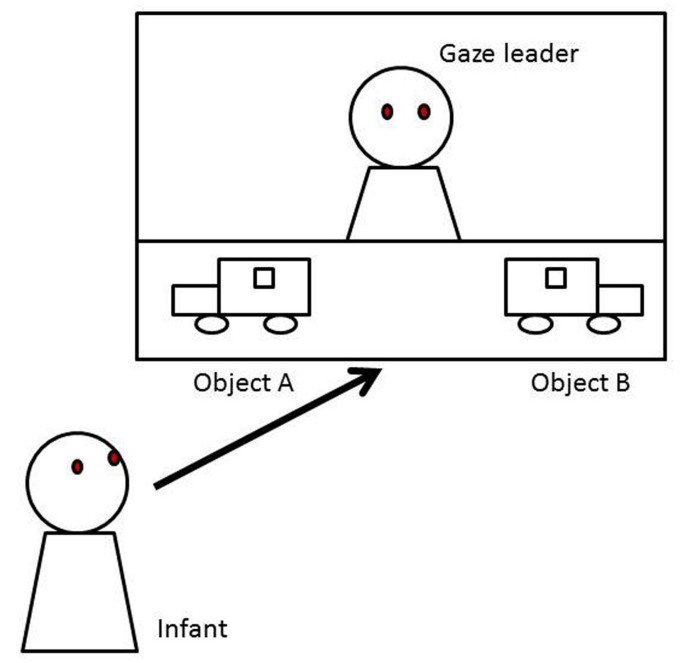

B

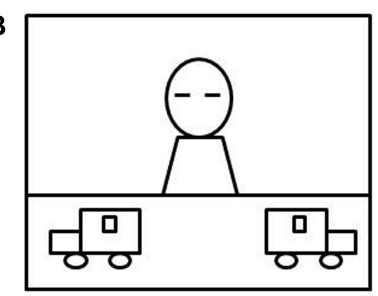

C

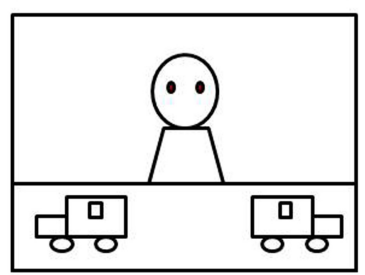

D

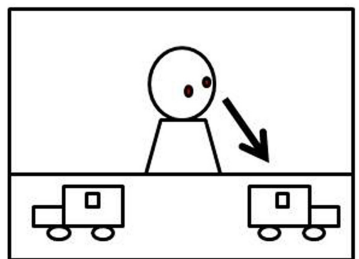

FIGURE 1 | Learning environment: an infant watches the situation with an actor and two objects (A). The actor closes his eyes in the initial phase (B). Next, the actor shows different actions such as opening his eyes (C) and then looks toward one of two objects (D).

TABLE 1 | Overview of model parameters and their allowed ranges.

\begin{tabular}{lll}
\hline Symbol & Explanation & Range \\
\hline$t$ & Number of trials & {$[1,2000]$} \\
$Q(A)$ & Behavioral value of random looking & $(0,1)$ \\
$Q(B)$ & Behavioral value of gaze following & $(0,1)$ \\
$P(A)$ & Probability of random looking & $(0,1)$ \\
$R$ & Reward value & 1 \\
$P(r)$ & Probability of reward & 0.5 or 1 \\
alpha & Learning rate & 0.005 \\
$S$ & Infant state & {$[0,1]$} \\
$D s$ & Infant default state & {$[0,1]$} \\
$C$ & Other's communicative intent & {$[0.5,1.5]$} \\
$M$ & Motivation for communication & constant \\
\hline
\end{tabular}

The formula with communicative cues is shown in (2):

$$
Q_{[t+1]}=Q_{[t]}+\alpha \times\left(R \times P_{[r]} \times C-Q_{[t]}\right)
$$

$C$ takes a random number between 0.5 (low communicative intent) and 1.5 (high communicative intent) in each trial. Because $C$ is an external factor and it is conceptually highly depending on context, $C$ was taken from a flat uniform distribution $($ mean $=1)$.

\section{Communicative Cue and Infant Internal State Model}

Infant attention has been argued to affect the perception and learning of the external environment (Rose et al., 1999; Tellinghuisen et al., 1999; Oakes et al., 2000). We set up that infant internal states modulate learning rate in QL. We added a variable named "S" (state) into the QL formula. The formula including infant internal states is shown in (3):

$$
Q_{[t+1]}=Q_{[t]}+\alpha \times S \times\left(R \times P_{[r]} \times C-Q_{[t]}\right)
$$

$S$ takes a random number between 0 (inattentive) and 1 (highly attending) in each trial. $S$ reflects an internal state, and it should be stable around resting state most of the time; therefore, $S$ was taken from a normal distribution (mean $=0.5, \sigma=0.16$ ). $S$ modulates the learning rate so it cannot exceed 1 because $\alpha$ is the limit of infant leaning in a trial.

\section{Communicative Cues Enhancing the Infant Internal State Model}

Formula (3) postulates that communicative cues and the infant internal state are independent of each other. In addition, we simulated a model that communicative cues enhance infant internal states, following the finding of Ishikawa and Itakura (2019).

To simulate that, we set up the "Default state" (Ds), which is the infant internal state before the effect of communicative cues. Ds takes a random number between 0 (inattentive) and 1 (highly attending) from a normal distribution (mean $=0.5, \sigma=0.16$ ) in each trial. Here, $S$ takes a number which is Ds modulated by $C$ (Figure 2). If $C$ is less than $1, S$ will be $D s \times 1$. Otherwise, if $C$ is greater than $1, S$ will be Ds $\times C$. Ishikawa and Itakura (2019) indicated that communicative cues enhance infant physiological arousal, but "no cues" do not affect the infant internal state. Therefore, we set up that if $C$ is less than median (1), the default state is not modulated. In addition, we posited that if Ds $\times C$ is 


\section{Communicative cues enhancing the infant internal state model}

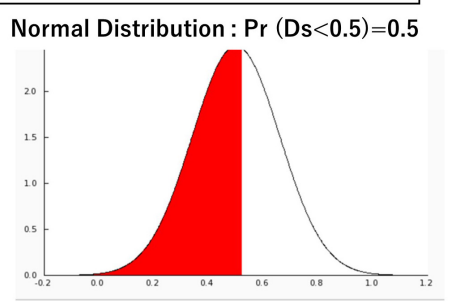

Individual differences

Normal Distribution : $\operatorname{Pr}(\mathrm{Ds}<0.5)=0.5$

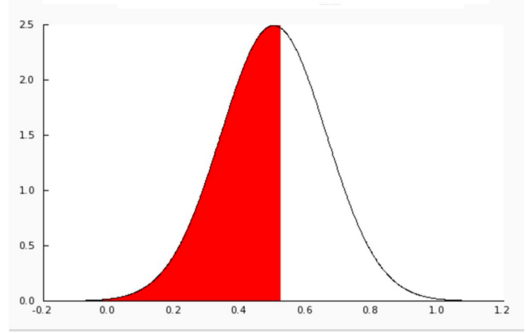

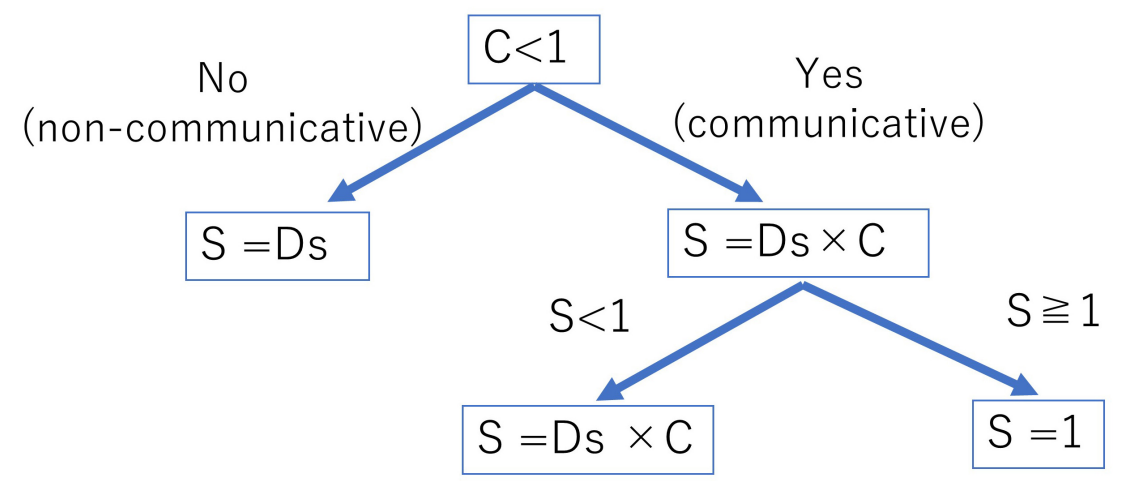

\begin{tabular}{c|c|c} 
No & $\mathrm{C}<1$ \\
(non-communicative) & Yes \\
$\mathrm{S}=\mathrm{Ds}$ & $\mathrm{S}=\mathrm{Ds} \times(\mathrm{C}+\mathrm{M})$
\end{tabular}

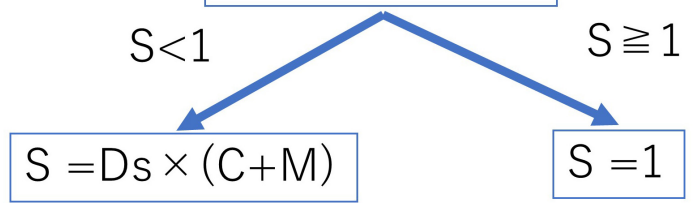

FIGURE 2 | Decision tree of variable model parameters.

more than $1, S$ will be 1 . As mentioned above, $S$ modulates the learning rate so it cannot exceed 1 because $\alpha$ is the limit of infant learning in a trial; so, if Ds $\times C$ is more than $1, S$ takes 1 .

\section{EXPERIMENT 1 RESULTS AND DISCUSSION}

Each experiment starts with all weights set to zero, and the models are simulated for a total of 2,000 time steps. The results are shown in Figure 3. All of these models were set up not to affect the optimal value of gaze following, thus all behavioral values after convergence were the same. In the model which only contains communicative cues, infant learning progressed at the same rate because infant internal state was kept constant through the simulation (Figure 3A, middle panel). Therefore, with high communicative intent, behavioral value drastically increased and the learning process was the most efficient. In the Q-learning, the speed of convergence means time taken to find a nearoptimal behavioral choice. The behavioral value of gaze following was converged around 1,600 trials (Figure 3A, middle panel). However, infant internal states were kept at the same level in this simulation, which would fail to simulate more realistic infant behavior affected by internal states.

With the addition of the infant internal state which is independent from communicative cues, the convergence of behavioral value took over 2,000 trials (Figure 3B, middle panel).
Here, infant internal states affected the learning rate. Therefore, although communicative cues were presented, if the infants were inattentive, they did not learn the behavioral value so much. In this model, infant learning is highly dependent on infant internal states.

In the model in which communicative cues enhanced infant internal states, the learning process was more efficient, converging around 1,750 trials (Figure 3C, middle panel). From the perspective of learning efficiency, the model with only communicative cues was the most efficient, but given that infant internal states are highly unlikely to be constant, the third model may be the most feasible to explain infant gaze following. Support for this model could also come from a recent empirical study that communicative cues can enhance the infant physiological state (Ishikawa and Itakura, 2019).

In addition, the Supplementary Material shows the learning process within 100 trials to observe how these models affect the short-term learning process. Communicative cues can directly modulate the subjective expected value, and they drastically update behavioral value in a very short term. On the other hand, if infant internal states change trial by trial, the behavioral value is updated gradually. Also, in the model with communicative cues enhancing states, the learning rate is increased in a trial with a high expected value, and, as a consequence, behavioral value was observed to be enhanced soon.

This simulation shows a possible learning process of gaze following. As a result, the model with communicative cues 

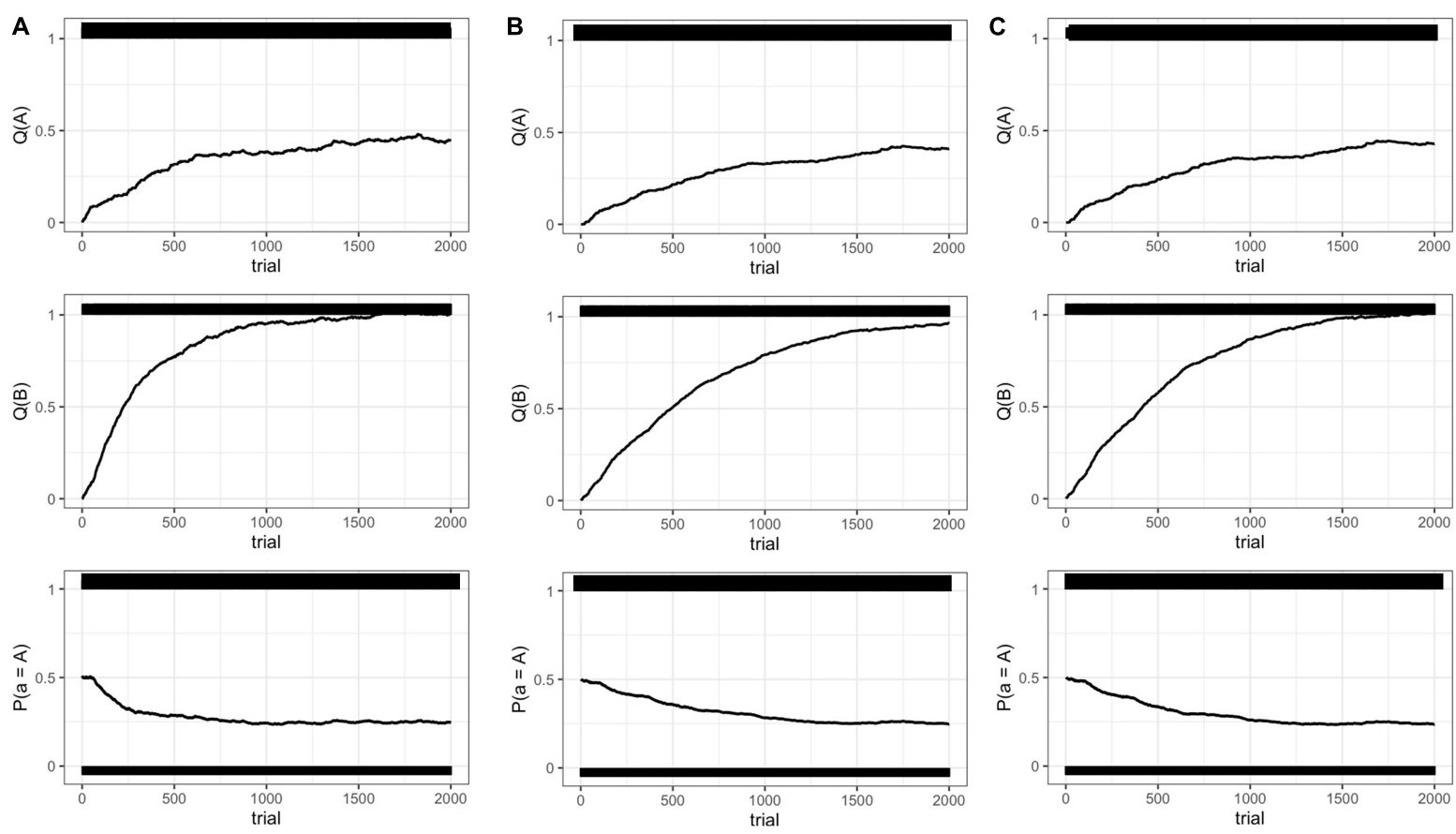

FIGURE 3 | The results of Experiment 1 in 2,000 steps. (A) Communicative cue model. (B) Communicative cue and infant internal state model. (C) Communicative cue enhancing infant internal state model. $Q(A)$ the behavioral value of random looking; $Q(B)$ the behavioral value of gaze following; $P(A)$ the probability of random looking predicted by a soft-max strategy.

enhancing infant internal state can be considered the most feasible to describe infant learning. In this study, we indicated that communicative cues may affect learning drastically in the short term; however, they would not affect the emergence of gaze following so much after the behavioral value was converged. Notably, after the behavioral value of gaze following was converged, it was predicted that the infants followed the other's gaze about 75\%, regardless of communicative cues (Figure 3, lower panels). It is consistent with Gredebäck et al. (2018) who showed that infants show gaze following in situations both with and without communicative cues. These results of the simulation demonstrate that a prior history of social learning, either within the experimental context or in real-life experience, may be an important factor in gaze following in empirical experiments.

In Experiment 1, we posited the learning process of gaze following in the experimental setting used in many empirical studies but did not include one important factor: individual difference. It has been reported that individual differences of gaze following can be observed in experimental situations (Morales et al., 1998, 2000; Brooks and Meltzoff, 2005). In Experiment 2, we applied the model with communicative cues enhancing states to simulate individual differences of gaze following.

\section{EXPERIMENT 2: MODELING INDIVIDUAL DIFFERENCES}

There is some evidence indicating individual differences of gaze following (see the review by Frischen et al., 2007). For example, sex differences in the sensitivity to other people's eye gaze can be detected from an early developmental stage. Lutchmaya et al. (2002) showed that in 12-month-old infants, male infants made less eye contact than female infants. Also, male infants looked toward faces less than females (Connellan et al., 2000; Lutchmaya and Baron-Cohen, 2002).

Also, autistic-like traits correlated with gaze cueing effects (Bayliss and Tipper, 2005; Bayliss et al., 2005). Children with autism spectrum disorder (ASD) are inattentive to social stimuli, and this means that they may lack adequate social learning experiences (Mundy and Neal, 2000; Schultz, 2005). One possible explanation for this phenomenon is the atypical development in social motivation in ASD (Chevallier et al., 2012). Some also argue that atypical development in social motivation in individuals with ASD is related to the atypical development of social reward processing (Bartz et al., 2011; Modi and Young, 2012; Dubey et al., 2015), although others did not find differences in the reinforcement value of social stimuli between individuals with and without ASD (e.g., Ewing et al., 2013; Vernetti et al., 2018). Overall, there is a theoretical and clinical interest in the possible influence of social motivation on the development of social attention and learning.

Here, we posit individual differences of motivation for communication. For example, autistic people and patients with social anxiety actively avoid to make eye contacts (Corden et al., 2008; Wieser et al., 2009). Thus, motivation for communication could be negative. We added a variable named " $M$ " (motivation) into the model with communicative cues enhancing states. $M$ can be considered to affect subjective evaluation of communicative 
cues; therefore, it modulates $C$ directly (Figure 2). If $C+M$ is less than $1, S$ will be Ds $\times 1$. Otherwise, if $C+M$ is more than $1, S$ will be $\mathrm{Ds} \times(C+M)$. In addition, if $\mathrm{Ds} \times(C+M)$ is more than $1, S$ will be 1 . The formula including infant internal states is described as follows in (4):

$$
Q_{[t+1]}=Q_{[t]}+\alpha \times S \times\left(R \times P_{[r]} \times(C+M)-Q_{[t]}\right)
$$

$M$ is a fixed number because motivation for communication can be considered as a trait for each individual. If $M$ takes a negative value, it means that infants actively avoid communication. On the other hand, if $M$ takes a positive value, infants engage communication more than average. Therefore, when infants have standard motivation for communication, $M$ takes 0 in this model. Here, we examined how the individual differences in $M$ affect the updating of gaze-following value.

\section{EXPERIMENT 2 RESULTS AND DISCUSSION}

The model was simulated for a total of 2,000 time steps. The results are shown in Figure 4.

Because behavioral values are converged to expected optimal values in Q-learning, the convergent value of gaze following was modulated by the degree of $M$. With $M$ less than 0 (low motivation for communication), the subjective value of gaze following was undervalued and the expected reward value was decreased (Figures 4A,B, middle panels). For example, when M takes -0.2 , the expected reward value of gaze-following behavior becomes 0.8 , and the convergent value is also decreased to 0.8 (Figure 4A, middle panels). As a result, the probability of gaze following was also decreased slightly $(M=-0.2$ : $P(\mathrm{~B})=70 \%$, Figure 4A, lower panel).

Here, we posit that motivation for communication may be behind individual differences in gaze following. Twomey and Westermann's (2018) infant computational modeling study suggested that infants are intrinsically motivated to select information that maximizes learning. In the context of learning in gaze following, it can be considered that interacting with others would maximize information to learn about the environment. For example, because gaze direction can help infants to associate words and objects, the development of gaze following affects later language development (Morales et al., 1998; Brooks and Meltzoff, 2005). Therefore, low motivation for communication can delay the social learning process in infants.

\section{GENERAL DISCUSSION}

In our simulation, the model, in which communicative cues affect infant internal states, emerged as the most feasible to explain infant gaze following. It shows more efficient learning than the model in which infant internal states are independent of communicative cues, and it shows comparable efficiency with the less realistic model which assumes that infant internal states are constant. The model is also consistent with the

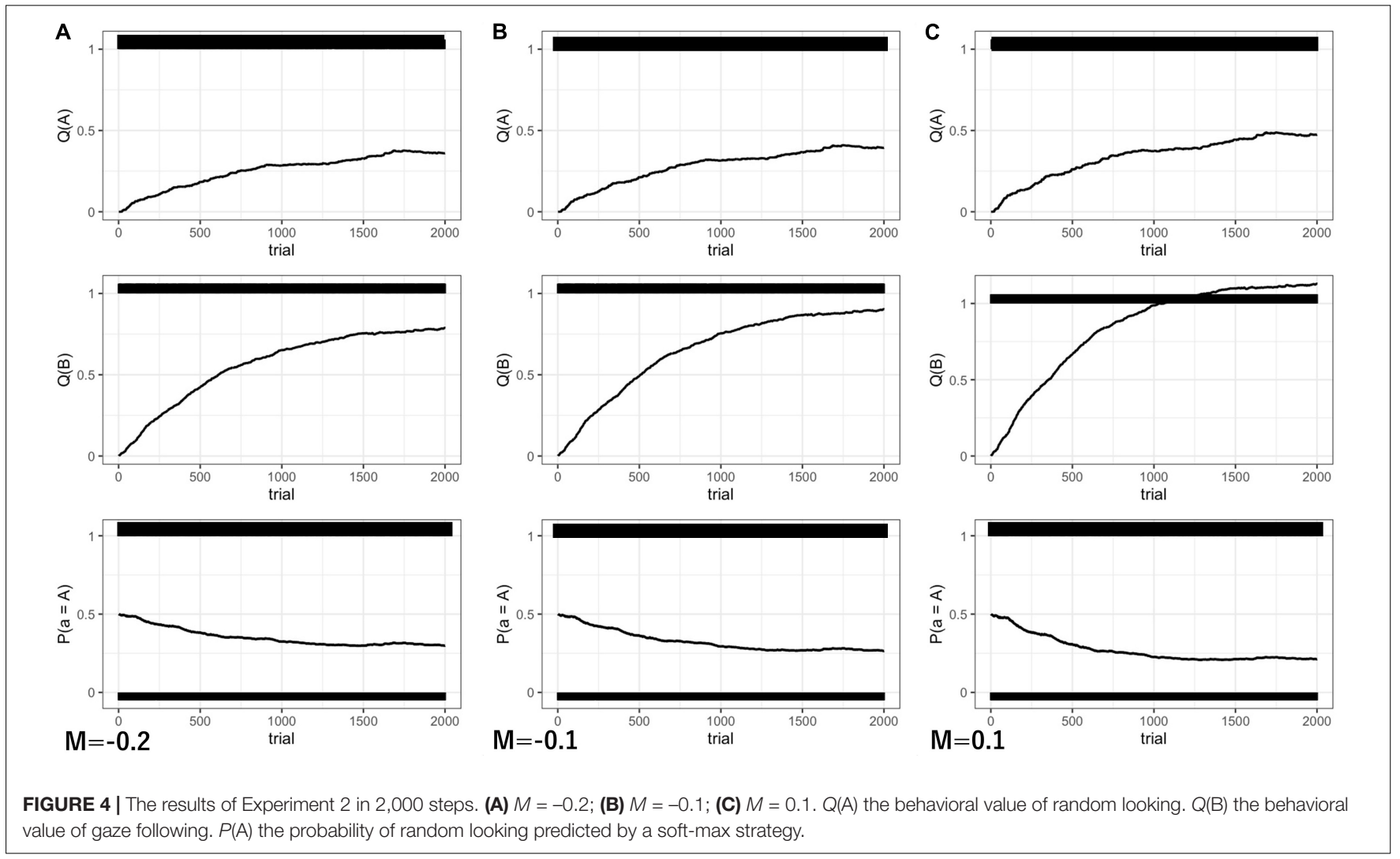


results of empirical research which examined the relation between communicative cues and infant physiological states (Ishikawa and Itakura, 2019).

The model hypothesizes that physiological arousal, at least partially, mediates the influence of ostensive signals on infant gaze following. The theory of natural pedagogy claims that human communication makes it possible to efficiently convey knowledge; in other words, communication can promote social learning (Csibra and Gergely, 2011). As communicative cues such as eye contact elevate physiological arousal (Nicholls and Champness, 1971; Helminen et al., 2011; Hietanen, 2018), and high physiological arousal is hypothesized to promote the learning process and enhances memory (Kleinsmith and Kaplan, 1963; Eysenck, 1976) particularly memory consolidation (LaBar and Phelps, 1998), infant learning may be more efficient with communicative cues partly because it enhances infant internal states, which we observe as physiological arousal.

Our model also showed that low motivation for communication can delay the learning process of gaze following. The model thus suggests that individuals with low social motivation, possibly including those with ASD, may be delayed in the learning process of gaze following. It is consistent with an empirical study reporting that neural sensitivity to dynamic eye gaze in infants aged 6-10 months old is associated with later emerging autism (Elsabbagh et al., 2012), even though they show typical gaze-following behavior (Bedford et al., 2012). Further, gaze following in infants predicts later language development and theory of mind skills (Brooks and Meltzoff, 2005, 2015). Initial social motivation may affect the learning process of gaze following, and as a result, the development of other social cognitions might also be affected.

Through the experiments, we simulated how infants' learning process of gaze following is affected by communicative cues, infant internal states, and social motivation. Our model was designed to offer simple simulation with reinforcement learning. Thus, the decision process of gaze following was only dependent on learned behavioral value. Following the results for the prediction of gaze following, after the behavioral value was converged, it can be predicted that gaze following emerges $75 \%$ in any situation, with or without communicative cues. It is consistent with Gredebäck et al. (2018), who indicated that infants show gaze following more than chance level (50\%) in all experimental situations with or without communicative cues, and they suggested that infant gaze following is not dependent on communicative cues. Our simulation, consistent with the results of Gredebäck et al. (2018), indicates that after infants have experienced a sufficient number of gazefollowing situations, infants tend to follow the other's gaze direction with or without preceding ostensive signals. Effects of communicative cues on social interactions have been mainly studies in infants. However, some studies have shown that toddlers can understand other's communicative intentions without ostensive signals such as eye contact and infant-directedspeech (IDS) (e.g., Moore et al., 2013). Social experiences in development may affect the engagement of interactions without communicative cues.
Note that the model has not accounted for other contextual information which affects gaze following, such as the other's social status, familiarity, facial expressions, or object pleasantness (Deaner et al., 2006; Dalmaso et al., 2011; Kuhn and Tipples, 2011). It is crucial for future studies to include more generalized contextual modulation because a recent study suggested that infants could use contextual information to guide their visual attention (Tummeltshammer and Amso, 2018). Further theoretical work is needed, which can account for how human infants, as well as older children and adults, decide to follow the other's gaze direction based on many kinds of contextual information to fully describe, explain, and predict gaze following in more naturalistic settings. Another limitation of this study is a lack of empirical data of developmental trajectory. This study theoretically simulated how external and internal factors affect the learning process of gaze following and chose a feasible model according to results of empirical studies in experimental settings. Computational modeling is useful to theoretically simulate and observe how behavioral models work; however, it cannot decide which models capture the development in real-world situations. To understand the development of gaze following, it is necessary to compare with longitudinal data measuring gazefollowing behavior.

To conclude, the results of the simulation presented in this paper suggest that the model in which communicative cues affect infant internal states is feasible to describe the learning process of gaze following. Also, with this reinforcement learning model, we succeeded in simulating how social motivation affects the development of gaze following and showed that low motivation for communication delayed the learning process of gaze following. In future works, other factors affecting the learning mechanism should also be included (e.g., tolerance to reversal learning). Computational modeling of the experimental setting can be helpful to give new insights into gaze following in infants.

\section{DATA AVAILABILITY STATEMENT}

The datasets generated for this study are available on request to the corresponding author.

\section{AUTHOR CONTRIBUTIONS}

MI designed the study, conducted computer simulation, and wrote the initial draft of the manuscript. All authors have contributed to interpretation, critically reviewed the manuscript, and approved the final version of the manuscript.

\section{SUPPLEMENTARY MATERIAL}

The Supplementary Material for this article can be found online at: https://www.frontiersin.org/articles/10.3389/fpsyg.2020. 00213/full\#supplementary-material 


\section{REFERENCES}

Aston-Jones, G., Chiang, C., and Alexinsky, T. (1991). "Discharge of noradrenergic locus coeruleus neurons in behaving rats and monkeys suggests a role in vigilance," in Progress in Brain Research, Vol. 88, eds C. D. Barnes and O. Pompeiano (Amsterdam: Elsevier), 501-520. doi: 10.1016/s0079-6123(08) 63830-3

Aston-Jones, G., Rajkowski, J., and Cohen, J. (1999). Role of locus coeruleus in attention and behavioral flexibility. Biol. Psychiatry 46, 1309-1320. doi: 10.1016/ S0006-3223(99)00140-7

Bartz, J. A., Zaki, J., Bolger, N., and Ochsner, K. N. (2011). Social effects of oxytocin in humans: context and person matter. Trends Cogn. Sci. 15, 301-309. doi: 10.1016/j.tics.2011.05.002

Bayliss, A. P., Pellegrino, G. D., and Tipper, S. P. (2005). Sex differences in eye gaze and symbolic cueing of attention. Q. J. Exp. Psychol. 58, 631-650. doi: 10.1080/02724980443000124

Bayliss, A. P., and Tipper, S. P. (2005). Gaze and arrow cueing of attention reveals individual differences along the autism spectrum as a function of target context. Br. J. Psychol. 96, 95-114. doi: 10.1348/000712604X15626

Bedford, R., Elsabbagh, M., Gliga, T., Pickles, A., Senju, A., Charman, T., et al. (2012). Precursors to social and communication difficulties in infants at-risk for autism: gaze following and attentional engagement. J. Autism Dev. Disord. 42, 2208-2218. doi: 10.1007/s10803-012-1450-y

Brooks, R., and Meltzoff, A. N. (2005). The development of gaze following and its relation to language. Dev. Sci. 8, 535-543. doi: 10.1111/j.1467-7687.2005.00 445.x

Brooks, R., and Meltzoff, A. N. (2015). Connecting the dots from infancy to childhood: a longitudinal study connecting gaze following, language, and explicit theory of mind. J. Exp. Child Psychol. 130, 67-78. doi: 10.1016/j.jecp. 2014.09.010

Butterworth, G., and Jarrett, N. (1991). What minds have in common is space: Spatial mechanisms serving joint visual attention in infancy. Br. J. Dev. Psychol. 9, 55-72. doi: 10.1111/j.2044-835X.1991.tb00862.x

Chevallier, C., Kohls, G., Troiani, V., Brodkin, E. S., and Schultz, R. T. (2012). The social motivation theory of autism. Trends Cogn. Sci. 16, 231-239. doi: 10.1016/j.tics.2012.02.007

Connellan, J., Baron-Cohen, S., Wheelwright, S., Batki, A., and Ahluwalia, J. (2000). Sex differences in human neonatal social perception. Infant Behav. Dev. 23, 113-118. doi: 10.1016/S0163-6383(00)00032-1

Corden, B., Chilvers, R., and Skuse, D. (2008). Avoidance of emotionally arousing stimuli predicts social-perceptual impairment in Asperger's syndrome. Neuropsychologia 46, 137-147. doi: 10.1016/j.neuropsychologia.2007. 08.005

Critchley, H. D., Rotshtein, P., Nagai, Y., O’Doherty, J., Mathias, C. J., and Dolan, R. J. (2005). Activity in the human brain predicting differential heart rate responses to emotional facial expressions. Neuroimage 24, 751-762. doi: 10 . 1016/j.neuroimage.2004.10.013

Csibra, G. (2010). Recognizing communicative intentions in infancy. Mind Lang. 25, 141-168. doi: 10.1111/j.1468-0017.2009.01384.x

Csibra, G., and Gergely, G. (2009). Natural pedagogy. Trends Cogn. Sci. 13, 148-153. doi: 10.1016/j.tics.2009.01.005

Csibra, G., and Gergely, G. (2011). Natural pedagogy as evolutionary adaptation. Philos. Trans. R. Soc. B Biol. Sci. 366, 1149-1157. doi: 10.1098/rstb.2010.0319

Dalmaso, M., Pavan, G., Castelli, L., and Galfano, G. (2011). Social status gates social attention in humans. Biol. Lett. 8, 450-452. doi: 10.1098/rsbl.2011.0881

Dayan, P., Abbott, L. F., and Abbott, L. (2001). Theoretical Neuroscience: Computational and Mathematical Modeling of Neural Systems. Cambridge, MA: MIT Press.

de Barbaro, K., Clackson, K., and Wass, S. V. (2017). Infant attention is dynamically modulated with changing arousal levels. Child Dev. 88, 629-639. doi: 10.1111/ cdev.12689

Deaner, R. O., Shepherd, S. V., and Platt, M. L. (2006). Familiarity accentuates gaze cuing in women but not men. Biol. Lett. 3, 65-68. doi: 10.1098/rsbl.2006.0564

D'Entremont, B., Hains, S. M., and Muir, D. W. (1997). A demonstration of gaze following in 3-to 6-month-olds. Infant Behav. Dev. 20, 569-572. doi: 10.1016/ S0163-6383(97)90048-5
Dubey, I., Ropar, D., and de C Hamilton, A. F. (2015). Measuring the value of social engagement in adults with and without autism. Mol. Autism 6:35. doi: 10.1186/s13229-015-0031-2

Elsabbagh, M., Mercure, E., Hudry, K., Chandler, S., Pasco, G., Charman, T., et al. (2012). Infant neural sensitivity to dynamic eye gaze is associated with later emerging autism. Curr. Biol. 22, 338-342. doi: 10.1016/j.cub.2011.12.056

Ewing, L., Pellicano, E., and Rhodes, G. (2013). Using effort to measure reward value of faces in children with autism. PLoS One. 8:e79493. doi: 10.1371/journal. pone. 0079493

Eysenck, M. W. (1976). Arousal, learning, and memory. Psychol. Bull. 83, 389-404. doi: 10.1037/0033-2909.83.3.389

Farroni, T., Csibra, G., Simion, F., and Johnson, M. H. (2002). Eye contact detection in humans from birth. Proc. Natl. Acad. Sci. U.S.A. 99, 9602-9605. doi: 10.1073/ pnas.152159999

Farroni, T., Massaccesi, S., Pividori, D., and Johnson, M. H. (2004). Gaze following in newborns. Infancy 5, 39-60. doi: 10.1207/s15327078in0501_2

Floccia, C., Christophe, A., and Bertoncini, J. (1997). High-amplitude sucking and newborns: the quest for underlying mechanisms. J. Exp. Child Psychol. 64, 175-198. doi: 10.1006/jecp.1996.2349

Frischen, A., Bayliss, A. P., and Tipper, S. P. (2007). Gaze cueing of attention: visual attention, social cognition, and individual differences. Psychol. Bull. 133, 694-724. doi: 10.1037/0033-2909.133.4.694

Gredebäck, G., Astor, K., and Fawcett, C. (2018). Gaze following is not dependent on ostensive cues: a critical test of natural pedagogy. Child Dev. 89, 2091-2098. doi: $10.1111 /$ cdev.13026

Gredebäck, G., Fikke, L., and Melinder, A. (2010). The development of joint visual attention: a longitudinal study of gaze following during interactions with mothers and strangers. Dev. Sci. 13, 839-848. doi: 10.1111/j.1467-7687.2009. 00945.x

Helminen, T. M., Kaasinen, S. M., and Hietanen, J. K. (2011). Eye contact and arousal: the effects of stimulus duration. Biol. Psychol. 88, 124-130. doi: 10.1016/ j.biopsycho.2011.07.002

Holroyd, C. B., and Coles, M. G. (2002). The neural basis of human error processing: reinforcement learning, dopamine, and the error-related negativity. Psychol. Rev. 109, 679-709. doi: 10.1037/0033-295X.109. 4.679

Hietanen, J. K. (2018). Affective eye contact: an integrative review. Front. Psychol. 9:1587. doi: 10.3389/fpsyg.2018.01587

Hernik, M., and Broesch, T. (2019). Infant gaze following depends on communicative signals: an eye-tracking study of 5- to 7-month-olds in Vanuatu. Dev. Sci. 22:e12779. doi: 10.1111/desc.12779

Ishikawa, M., and Itakura, S. (2019). Physiological arousal predicts gaze following in infants. Proc. Biol. Sci. 286:20182746. doi: 10.1098/rspb.2018.2746

Johnson, M. H., Dziurawiec, S., Ellis, H., and Morton, J. (1991). Newborns' preferential tracking of face-like stimuli and its subsequent decline. Cognition 40, 1-19. doi: 10.1016/0010-0277(91)90045-6

Kleinsmith, L. J., and Kaplan, S. (1963). Paired-associate learning as a function of arousal and interpolated interval. J. Exp. Psychol. 65, 190-193. doi: 10.1037/ h0040288

Kuhn, G., and Tipples, J. (2011). Increased gaze following for fearful faces. It depends on what you're looking for!. Psychon. Bull. Rev. 18, 89-95. doi: 10.3758/ s13423-010-0033-1

LaBar, K. S., and Phelps, E. A. (1998). Arousal-mediated memory consolidation: role of the medial temporal lobe in humans. Psychol. Sci. 9, 490-493. doi: 10.1111/1467-9280.00090

Lutchmaya, S., and Baron-Cohen, S. (2002). Human sex differences in social and non-social looking preferences, at 12 months of age. Infant Behav. Dev. 25, 319-325. doi: 10.1016/S0163-6383(02)00095-4

Lutchmaya, S., Baron-Cohen, S., and Raggatt, P. (2002). Foetal testosterone and eye contact in 12-month-old human infants. Infant Behav. Dev. 25, 327-335. doi: 10.1016/S0163-6383(02)00094-2

Modi, M. E., and Young, L. J. (2012). The oxytocin system in drug discovery for autism: animal models and novel therapeutic strategies. Horm. Behav. 61, 340-350. doi: 10.1016/j.yhbeh.2011.12.010

Moore, C., and Corkum, V. (1994). Social understanding at the end of the first year of life. Dev. Rev. 14, 349-372. doi: 10.1006/drev.1994.1014 
Moore, R., Liebal, K., and Tomasello, M. (2013). Three-year-olds understand communicative intentions without language, gestures, or gaze. Interact. Stud. 14, 62-80. doi: 10.1075/is.14.1.05moo

Morales, M., Mundy, P., Delgado, C. E., Yale, M., Neal, R., and Schwartz, H. K. (2000). Gaze following, temperament, and language development in 6-montholds: a replication and extension. Infant Behav. Dev. 23, 231-236. doi: 10.1016/ S0163-6383(01)00038-8

Morales, M., Mundy, P., and Rojas, J. (1998). Following the direction of gaze and language development in 6-month-olds. Infant Behav. Dev. 21, 373-377. doi: 10.1016/S0163-6383(98)90014-5

Mundy, P. (1995). Joint attention and social-emotional approach behavior in children with autism. Dev. Psychopathol. 7, 63-82. doi: 10.1017/ S0954579400006349

Mundy, P., and Neal, A. R. (2000). "Neural plasticity, joint attention, and a transactional social-orienting model of autism," in International Review of Research in Mental Retardation, Vol. 23, ed. L. M. Glidden (Cambridge, MA: Academic Press), 139-168. doi: 10.1016/s0074-7750(00)80009-9

Nicholls, K. A., and Champness, B. G. (1971). Eye gaze and GSR. J. Exp. Soc. Psychol. 7, 623-626. doi: 10.1016/0022-1031(71)90024-2

Oakes, L. M., Tellinghuisen, D. J., and Tjebkes, T. L. (2000). Competition for infants' attention: the interactive influence of attentional state and stimulus characteristics. Infancy 1, 347-361. doi: 10.1207/S15327078IN0103_4

Rose, S. A., Futterweit, L. R., and Jankowski, J. J. (1999). The relation of affect to attention and learning in infancy. Child Dev. 70, 549-559. doi: 10.1111/14678624.00040

Schultz, R. T. (2005). Developmental deficits in social perception in autism: the role of the amygdala and fusiform face area. Int. J. Dev. Neurosci. 23, 125-141. doi: 10.1016/j.ijdevneu.2004.12.012

Senju, A., and Csibra, G. (2008). Gaze following in human infants depends on communicative signals. Curr. Biol. 18, 668-671. doi: 10.1016/j.cub.2008.03.059

Sutton, R. S. (1988). Learning to predict by the methods of temporal differences. Mach. Learn. 3, 9-44. doi: 10.1007/BF00115009

Sutton, R. S., and Barto, A. G. (1998). Reinforcement Learning: An Introduction. Cambridge, MA: The MIT Press.

Szufnarowska, J., Rohlfing, K. J., Fawcett, C., and Gredebäck, G. (2014). Is ostension any more than attention? Sci. Rep. 4:5304. doi: 10.1038/srep05304

Tellinghuisen, D. J., Oakes, L. M., and Tjebkes, T. L. (1999). The influence of attentional state and stimulus characteristics on infant distractibility. Cogn. Dev. 14, 199-213. doi: 10.1016/S0885-2014(99) 00002-7
Triesch, J., Jasso, H., and Deák, G. O. (2007). Emergence of mirror neurons in a model of gaze following. Adapt. Behav. 15, 149-165. doi: 10.1177/ 1059712307078654

Triesch, J., Teuscher, C., Deák, G. O., and Carlson, E. (2006). Gaze following: why (not) learn it? Dev. Sci. 9, 125-147. doi: 10.1111/j.1467-7687.2006.00470.x

Tummeltshammer, K., and Amso, D. (2018). Top-down contextual knowledge guides visual attention in infancy. Dev. Sci. 21:e12599. doi: 10.1111/desc. 12599

Tummeltshammer, K., Feldman, E. C., and Amso, D. (2019). Using pupil dilation, eye-blink rate, and the value of mother to investigate reward learning mechanisms in infancy. Dev. Cogn. Neurosci. 36:100608. doi: 10.1016/j.dcn. 2018.12.006

Twomey, K. E., and Westermann, G. (2018). Curiosity-based learning in infants: a neurocomputational approach. Dev. Sci. 21:e12629. doi: 10.1111/desc.12629

Vernetti, A., Senju, A., Charman, T., Johnson, M. H., Gliga, T., and BASIS Team. (2018). Simulating interaction: using gaze-contingent eye-tracking to measure the reward value of social signals in toddlers with and without autism. Dev. Cogn. Neurosci. 29, 21-29. doi: 10.1016/j.dcn.2017.08.004

Valenza, E., Simion, F., Cassia, V. M., and Umiltà, C. (1996). Face preference at birth. J. Exp. Psychol Hum. Percept. Perform. 22, 892-903. doi: 10.1037/00961523.22.4.892

Wass, S. V., Clackson, K., and de Barbaro, K. (2016). Temporal dynamics of arousal and attention in 12-month-old infants. Dev. Psychobiol. 58, 623-639. doi: 10.1002/dev.21406

Watkins, C. J., and Dayan, P. (1992). Q-learning. Mach. Learn. 8, 279-292.

Wieser, M. J., Pauli, P., Alpers, G. W., and Mühlberger, A. (2009). Is eye to eye contact really threatening and avoided in social anxiety? An eye-tracking and psychophysiology study. J. Anxiety Disord. 23, 93-103. doi: 10.1016/j.janxdis. 2008.04.004

Conflict of Interest: The authors declare that the research was conducted in the absence of any commercial or financial relationships that could be construed as a potential conflict of interest.

Copyright (c) 2020 Ishikawa, Senju and Itakura. This is an open-access article distributed under the terms of the Creative Commons Attribution License (CC BY). The use, distribution or reproduction in other forums is permitted, provided the original author(s) and the copyright owner(s) are credited and that the original publication in this journal is cited, in accordance with accepted academic practice. No use, distribution or reproduction is permitted which does not comply with these terms. 
OPEN ACCESS

Edited by: Giulia Savarese, University of Salerno, Italy

Reviewed by:

Chunije Zhang

Beijing Jiaotong University, China

Masami lkeda

Jumonji University, Japan

*Correspondence:

Kosuke Taniguchi kosuket314@gmail.com

Specialty section: This article was submitted to

Developmental Psychology, a section of the journal Frontiers in Psychology

Received: 16 October 2019 Accepted: 02 March 2020

Published: 25 March 2020

Citation:

Taniguchi K, Kuraguchi K, Takano Y and Itakura S (2020) Object

Categorization Processing Differs According to Category Level: Comparing Visual Information Between the Basic and Superordinate Levels. Front. Psychol. 11:501. doi: 10.3389/fpsyg.2020.00501

\section{Object Categorization Processing Differs According to Category Level: Comparing Visual Information Between the Basic and Superordinate Levels}

\author{
Kosuke Taniguchi' ${ }^{1 *}$, Kana Kuraguchi' ${ }^{2}$, Yuji Takano ${ }^{3}$ and Shoji Itakura ${ }^{1}$ \\ ${ }^{1}$ Center for Baby Science, Doshisha University, Kyoto, Japan, ${ }^{2}$ Faculty of Psychology, Otemon Gakuin University, Osaka, \\ Japan, ${ }^{3}$ Smart-Aging Research Center, Tohoku University, Miyagi, Japan
}

Object category levels comprise a crucial concept in the field of object recognition. Specifically, categorization performance differs according to the category level of the target object. This study involved experiments with two types of stimulus sequences (i.e., forward condition: presenting the target name before the line-drawing stimulus; and reverse condition: presenting the target name after the line-drawing stimulus) for both basic- and superordinate-level categorizations. Adult participants were assigned to each level and asked to judge whether briefly presented stimuli included the same object and target name. Here, we investigated how the category level altered the categorization process. We conducted path analyses using a multivariate multiple regression model, and set our variables to investigate whether the predictors affected the categorization process between two types of stimulus sequence. Dependent variables included the measures of performance (i.e., reaction time, accuracy) for each categorization task. The predictors included dimensions and shapes of the line-drawings, such as primary and local shape information, shape complexity, subject estimation, and other shape variables related to object recognition. Results showed that the categorization process differed according to shape properties between conditions only for basic-level categorizations. For the forward condition, the bottom-up processing of primary visual information depended on matches with stored representations for the basic-level category. For the reverse condition at the basic-level category, decisions depended on subjective ratings in terms of object-representation accessibility. Finally, superordinate-level decisions depended on higher levels of visual information in terms of complexity, regardless of the condition. Thus, the given category level altered the processing of visual information for object recognition in relation to shape properties. This indicates that decision processing for object recognition is flexible depending on the criteria of the processed objects (e.g., category levels).

Keywords: object recognition, categorization, category level, line-drawings, visual processing, word-stimulus sequence 


\section{INTRODUCTION}

Object recognition is the foundation of various cognitive processes used in daily behavior (e.g., grabbing a cup, pointing at a target, or communicating with others). Patients with semantic dementia (a disease that affects conceptual knowledge regarding word and object meanings) may experience the deterioration of many other cognitive abilities during later stages (Rogers and Patterson, 2007). Individuals with Autism Spectrum Disorder (ASD) may also recognize objects in a different manner, thus potentially causing social difficulties (e.g., communication and behavioral deficits; Gastgeb and Strauss, 2012). To understand those who experience difficulty with object recognition, we must scrutinize the object recognition process in neurotypical adults who can judge single objects from a variety of category levels - a crucial component of object recognition. For example, most individuals who see a dog can categorize the animal as such at a glance, but can also more specifically categorize the dog (e.g., as a Siberian husky) both quickly and accurately. However, the visual information that is needed for categorization might differ between category levels (Mack and Palmeri, 2011, for psychology; Zhang et al., 2017, for computer vision). We, therefore, pose an important question: does object recognition adhere to the same process regardless of differences in the object category level? This study investigated how different category levels affected objectrecognition processing performance.

Object category level is known to alter decision speed during object recognition. Rosch et al. (1976) suggested that categorization reaction time was faster when recognizing an object at an intermediate (basic; i.e., dog) level than when recognizing it at both more general (superordinate; i.e., animal) and specific (subordinate; i.e., Siberian husky) category levels. The same study indicated that basic-level categorizations were the fastest because they were conducted according to representative features in the same category. That is, individuals discriminated such features from those in other category levels.

However, basic-level categorizations are not always faster than those conducted at other levels. For example, an atypical member is categorized faster at the subordinate level (e.g., penguin) than at the basic level (e.g., bird; Jolicoeur et al., 1984). Further, a study among bird and dog experts revealed that categorizations at the subordinate level (e.g., Java sparrow) were as quick and accurate as those conducted at the basic level (e.g., bird; Tanaka and Taylor, 1991; Johnson and Mervis, 1997). On the other hand, ultra-rapid categorization tasks conducted with very brief stimuli (e.g., less than $30 \mathrm{~ms}$ ) have shown that superordinate-level categorizations (e.g., animal) can be conducted more quickly than both basic(e.g., dog) and subordinate-level (e.g., terrier) categorizations (Thorpe et al., 1996; Macé et al., 2009).

The representative theory of object recognition posits that visual-object processing assumes a hierarchical structure involving at least three stages. First, visual information is received by the retina, analyzed, and divided into primary information groups (e.g., edge extraction, depth segmentation, surface texture, and color). Second, an object is extracted based on this primary information. Third, the object is recognized and categorized according to a top-down matching process in which the extracted object is compared with stored representations (Rubin, 1958; Biederman, 1987; Nakayama et al., 1995; Driver and Baylis, 1996). Differences in object category levels are assumed to reflect differences in the top-down matching process completed during the third stage. Different category-level response speeds are thus the result of how object representations are accessed.

Another possible interpretation is that the required visual information differs according to the category level. As noted above, visual object recognition depends on two-way processing (i.e., bottom-up and top-down matching). Previous studies in this context have provided participants with object category levels prior to receiving the stimulus (e.g., Grill-Spector and Kanwisher, 2005). Here, given category levels may have altered bottom-up processing. As such, these studies investigated how different category levels affected the visual information needed to categorize the target object. Johnson and Olshausen (2005) indicated that forward (in which the name of a target object is given prior to the stimulus) and reverse (in which the name of a target object is given after the stimulus) conditions lead to different matching processes during object recognition. Following this, we compared the accuracy and reaction times of object recognition tasks performed with forward and reverse conditions to identify the type of processing (i.e., bottom-up or top-down) that was more effective when participants judged objects in terms of basic- or superordinate-level categories.

Various visual information types (e.g., shape, color, and texture) are used during the object recognition process, but previous research has established that shape is the most important (Marr, 1982; Biederman, 1987; Ullman, 1996). As such, this study focused on shape-related information when conducting comparisons between object category levels. Here, the curvature extrema (i.e., maximum point of curvature reached locally in a concave or convex contour) were assumed to be the most important pieces of information. Attneave (1954) showed that the most salient points were found at convex and concave points in various object contours. The curvature extrema are also crucial during the object recognition process (cf. Norman et al., 2001). Other studies have indicated that inflection points (where the curvature goes through zero locally) are also essential for object recognition (Koenderink and van Doorn, 1982; Kennedy and Domander, 1985). The present study thus investigated whether different visual information was required for the basic and superordinate levels.

To summarize: this study specifically, (1) investigated whether decisions involving both basic- and superordinatelevel categorizations depended on bottom-up and/or top-down processing according to forward and reverse conditions, and (2) specified what visual information was required at each level (Figure 1). In this study, we measured both reaction time and accuracy to specify categorization processing. This is because Taniguchi et al. (2018) showed that object recognition involves inaccurate decision with fast reaction time and accurate decision with slow reaction time. Please note that this study did not attempt to access subordinate-level categorizations through line-drawing images due to the associated difficulty (Rosch et al., 1976). 


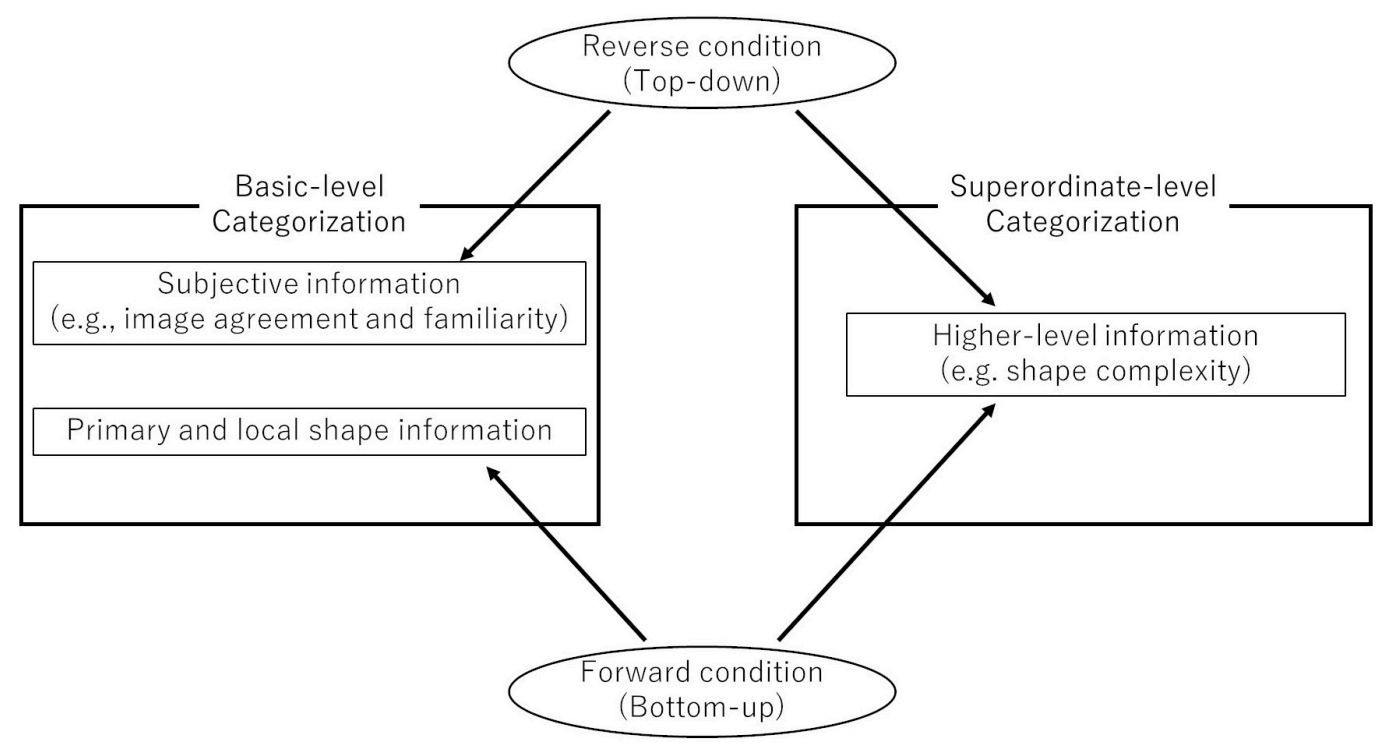

FIGURE 1 | Summary of the assumption and the results of this study.

\section{MATERIALS AND METHODS}

\section{Participants}

A total of 45 participants were selected for this study. This included 21 (eight males and 13 females aged 19-22 years) for the basic-level tasks and 24 (eight males and 16 females aged 20-28 years) for the superordinate-level tasks. All participants were Japanese undergraduate or graduate students, had normal or normal-corrected vision, and were unaware of the study's purpose. Informed consent was obtained from all participants before conducting the experiments. Each received 1,000 yen for their participation. The Ethical Committee of the Doshisha University approved of this research (15053).

\section{Stimuli}

Line-drawing images depicting animal, plant, clothing, furniture, musical instrument, and vehicle categories were used following a study by Snodgrass and Vanderwart (1980). A total of 40 images were presented (i.e., 10 animals, 10 plants, five clothing articles, five furniture types, five musical instruments, and five vehicles). We investigated whether these images could be recognized accurately in a pilot study and showed stimulus validation with more than $88 \%$ accuracy. The stimuli were created by removing approximately $90 \%$ of the black pixels from each image (Figure 2). Here, a 50\% level of black-and-white random noise was used for a mask. All stimuli were set at $8.86 \times 8.86$ degree of visual angles (400 $\times 400$ pixels). All stimuli and masks were presented at the center of a 16-inch cathode-ray tube (CRT) screen (Dell E771p) over a gray background. Observation distance was set at approximately 60 centimeters, and was maintained using a chin rest. The experiments were conducted in a dark, quiet room.

\section{Procedure}

Stimuli presentation alternated between forward and reverse conditions. The forward condition presented a (1) target name for $800 \mathrm{~ms}$, (2) fixation cross for $500 \mathrm{~ms}$, (3) stimulus for $150 \mathrm{~ms}$, and (4) mask for $100 \mathrm{~ms}$ (Figure 3A), while the reverse condition presented a (1) fixation cross for $500 \mathrm{~ms}$, (2) stimulus for $150 \mathrm{~ms}$, (3) mask for $100 \mathrm{~ms}$, and (4) target name for $800 \mathrm{~ms}$ (Figure 3B). Target names differed according to the basic- and superordinate-level tasks. Participants were asked to announce, as soon and as accurately as possible, whether the target name was consistent with or involved in the stimulus. During basic-level tasks, there were also distractor trials in which other stimuli in the same superordinate-level categories were randomly presented (e.g., when the target name was a dog, the image of a different animal - such as a cat or elephant - was displayed). During superordinate-level tasks, other stimuli in the same natural/artifactual categories were
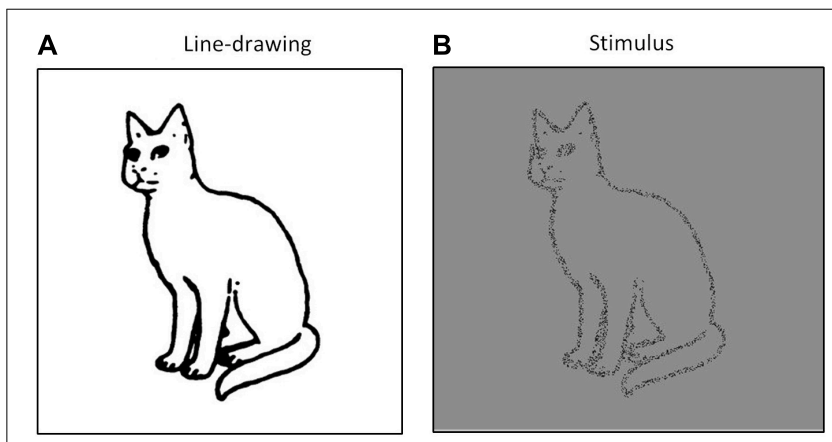

FIGURE 2 | Example of a stimulus image. (A) Original line-drawing from Snodgrass and Vanderwart (1980) and (B) the stimulus image used in this study. 


\section{A Forward conditon}

(1) Target-name

(2) Fixation

(3) Stimulus

(4) Mask

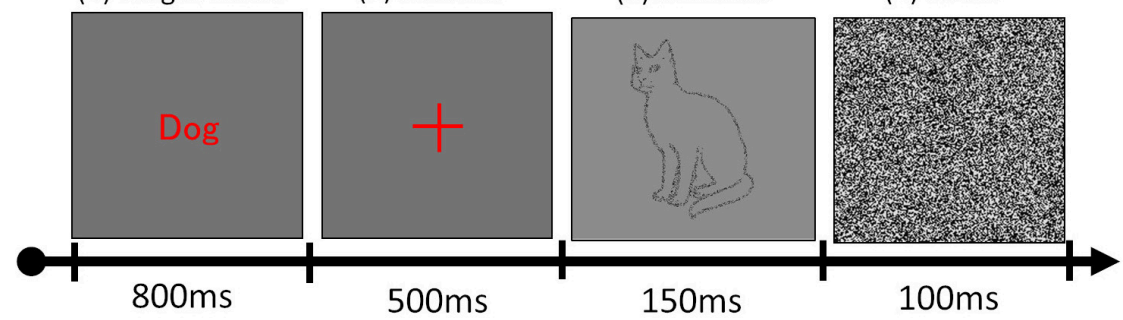

B Reverse conditon

(1) Fixation

(2) Stimulus

(3) Mask

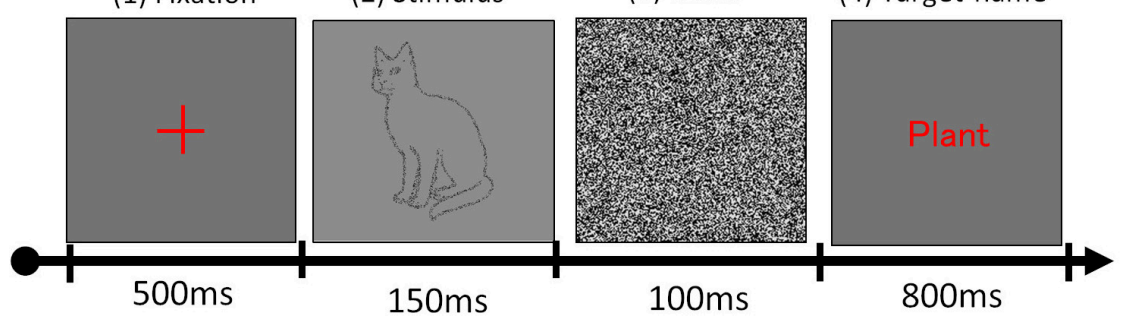

FIGURE 3 | Stimulus presentation sequence. (A) Forward and (B) reverse conditions used in the experiment.

also randomly presented (e.g., when the target was a clothing article, an object from a different category - such as a table or guitar - was displayed). Each object stimulus was presented twice for each condition (i.e., once as the target and once as the distractor). The experiments involved 80 total trials for each condition, consisting of 40 object images from six categories and congruent/incongruent trials of word-stimulus. All conditions were conducted using a blocked design and all trials were randomly assigned. Presentation and recording experiments were presented and recorded using the Windows 7 software. All participants completed eight practice trials before starting the formal experiment. The practice trials and formal tests were conducted identically, with one exception: participants were given advance knowledge of the object stimuli before starting the experiment. Each participant took approximately $30 \mathrm{~min}$ to complete the experiment.

\section{Shape Variables}

This study incorporated path analyses to examine whether the shape variables involved in and estimated from the linedrawing images affected accuracy and reaction times during basic- and superordinate-level categorizations. Shape variables were calculated through image analyses or obtained from previous studies (Snodgrass and Vanderwart, 1980; De Winter and Wagemans, 2008).

The shape variables used in this study consisted of basic information from the line-drawing images, indexes of shape complexities, and subjective evaluations. The number of black pixels were counted to reflect the basic properties of each linedrawing image (Pixels). The ratio of three black pixels aligned along rows or columns in the pixel matrix was then calculated to indicate horizontal and vertical components (Matrix), while the ratio of three black pixels aligned in slanted lines to the pixels slants were calculated to indicate the slant-line components (Slant). The number of curvature singularity positions (e.g., positive maxima $(\mathrm{M}+)$, negative minima ( $\mathrm{m}-)$, and inflections (I) corresponding to the positions of visual salience) was then used following De Winter and Wagemans (2008). Aspect ratio (AR) and degree of circularity (DC) were used as indices of shape complexity for the image analyses. AR refers to the ratio of horizontal and vertical contour lengths, while DC refers to the degree to which a given shape approximates circularity. Finally, subjective evaluations of visual complexity (Complexity), familiarity (Familiarity), and image agreement (ImageAgree) were used as shape variables, following Snodgrass and Vanderwart (1980).

\section{RESULTS}

For the trials that presented a bicycle-image stimulus, no data were collected due to a technical error. One participant was removed from each of the basic- and superordinate-level tasks and excluded from further analysis, in the first instance because mean reaction time was slower than 1,000 ms, and in the second because accuracy in the forward condition was nearly at chance level (50\%). Reaction times of less than $150 \mathrm{~ms}$ and greater than $1,846 \mathrm{~ms}$ (mean [793] + $3 \mathrm{SD}$ $[3 \times 351])$ were also excluded as anticipation errors and outliers, respectively. The mean accuracy was approximately 0.93 $(\mathrm{SD}=0.25)$ at basic level and $0.92(\mathrm{SD}=0.27)$ at superordinate level. The mean reaction time was approximately $625 \mathrm{~ms}$ $(\mathrm{SD}=218 \mathrm{~ms})$ at basic level and $705 \mathrm{~ms}(\mathrm{SD}=222 \mathrm{~ms})$ at superordinate level. 


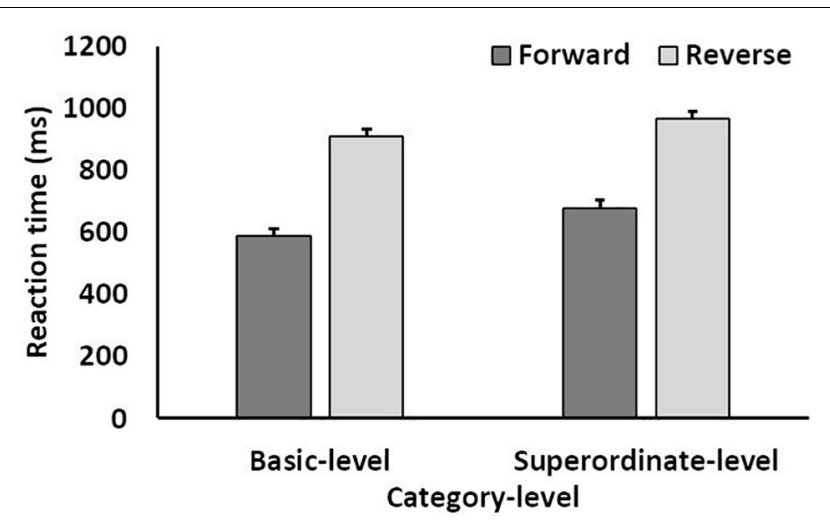

FIGURE 4 | Mean reaction time as a function of target-word position (forward vs. reverse condition) and category level (basic level vs. superordinate level). Error bars indicate standard errors of the means.

A three-way mixed ANOVA was conducted for reaction time [between factor: category level; within factors: target-word position (forward vs. reverse) and stimulus category]. Results indicated that the main effects of the category level and targetword position were significant (category level and target-word position: $\left.F(1,41)>8.25, p<0.01, \eta_{\mathrm{p}}^{2}>0.16\right)$. The interactions between the category level and target-word position were also significant $\left(F(1,41)=13.16, p<0.001, \eta_{\mathrm{p}}{ }^{2}=0.24\right)$. The main effects of the stimulus category and other interactions were insignificant. Further, an analysis of the simple effects between the category level and target-word position showed that all single main effects were significant. This indicates that reaction times for the forward condition were shorter than those for the reverse condition at both the basic and superordinate levels, while reaction times for the basic level were shorter than those for the superordinate level for both the forward and reverse conditions (Figure 4).

A three-way mixed ANOVA was also conducted for accuracy (between factor: category level; within factors: target-word position and stimulus category). Results showed that the main effects of the stimulus category and interactions between category level and stimulus category were significant (stimulus category: $F(5,205)=5.14, p<0.01, \eta_{\mathrm{p}}{ }^{2}=0.11, \varepsilon=0.74$; category level $\times$ stimulus category: $F(5,205)=7.33, p<0.001, \eta_{\mathrm{p}}{ }^{2}=0.15$, $\varepsilon=0.74)$. An analysis of the simple effect between the category level and stimulus category showed that the simple categorylevel effects for animals, plants, and furniture were significant $\left(F(1,41)>6.59, p<0.05, \eta_{\mathrm{p}}{ }^{2}>0.13\right)$. This indicates that accuracy was higher for the basic level than for the superordinate level for both animals and plants, whereas accuracy was higher for the superordinate level than for the basic-level for furniture. The simple main effect of the stimulus category was also significant at the basic level. Multiple comparisons indicated that the furniture category was recognized with less overall accuracy than the animal, plant, and clothing categories, while musical instruments were recognized with less accuracy than animals (Figure 5).

We then investigated the effects of visual properties on both reaction time and accuracy during categorization. This

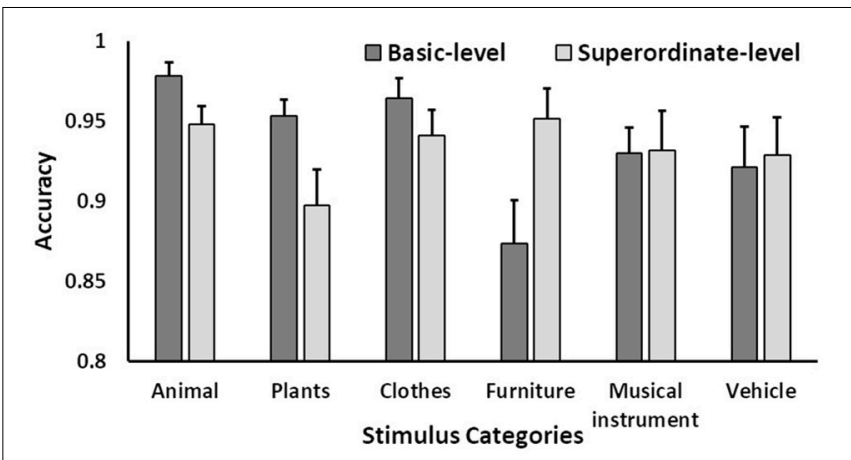

FIGURE 5 | Mean accuracy as a function of category level and stimulus categories. Error bars indicate standard errors of the means.

analysis was done according to a path analysis conducted with the sem package in $\mathrm{R}$. The path model set reaction times for forward and reverse tasks as dependent variables, and each shape (Pixels, Matrix, Slant, M+, m-, I, AR, DC, Complexity, ImageAgree, and Familiarity) as a predictive variable. We obtained the best fitting model by estimating a saturated model containing the causal paths of all 10 predictive variables to each dependent variable (i.e., forward and reverse), correlation paths among all predictive variables (i.e., shape variables; 45 total correlations), and a correlation path between forward and reverse. Path analyses were separately conducted for the basic and superordinate levels. Paths with the highest $p$-values were deleted from the model and re-estimated for a reduced model. We conducted this reduction procedure using a backward stepwise method until all paths were significant. We then chose the lowest Akaike information criterion (AIC) model from all those estimated.

The best-fitting path model for reaction time at the basic level is summarized in Figure 6A $\left(\chi^{2}(31)=11.296, p=0.999\right.$, $\mathrm{GFI}=0.958$, NFI $=0.976, \mathrm{CFI}=1.000, \mathrm{RMSEA}=0.000$, AIC $=131.296)$. Reaction time for the forward condition was significantly influenced by Pixels $(\beta=0.61, p<0.001)$, Matrix $(\beta=-0.50, p<0.01), \mathrm{M}+(\beta=-0.96, p<0.001)$, and $\mathrm{m}-$ $(\beta=0.47, p<0.05)$, while reaction time for the reverse condition was influenced by $\mathrm{M}+(\beta=-0.39, p<0.01)$, ImageAgree $(\beta=-0.24, p<0.05)$, and Familiarity $(\beta=-0.19, p<0.05)$. Further, significant Pixels $(\beta=0.63, p<0.001)$, AR $(\beta=0.67$, $p<0.001)$ and Complexity $(\beta=-0.47, p<0.01)$ effects were found on reaction time for the forward condition at the superordinate level, while marginally significant effects were found for Slant $(\beta=-0.29, p<0.1)$ and $\mathrm{DC}(\beta=0.35$, $p<0.1)$. The effects of Pixels $(\beta=0.60, p<0.01)$ and Complexity $(\beta=-0.50, p<0.01)$ were significant at the reverse condition, while effects of $\operatorname{AR}(\beta=0.32, p<0.1)$ were marginally significant (Figure 6B) $\left(\chi^{2}(44)=33.381, p=0.878, \mathrm{GFI}=0.890\right.$, $\mathrm{NFI}=0.909, \mathrm{CFI}=1.000, \mathrm{RMSEA}=0.000, \mathrm{AIC}=127.382)$.

For the best-fitting path model for accuracy at the basic level, the effects of Pixels $(\beta=-0.47, p<0.001)$, Slant $(\beta=0.27, p<0.01), M+(\beta=0.23, p<0.05)$, and ImageAgree $(\beta=0.44, p<0.01)$ were significant for the forward condition, 


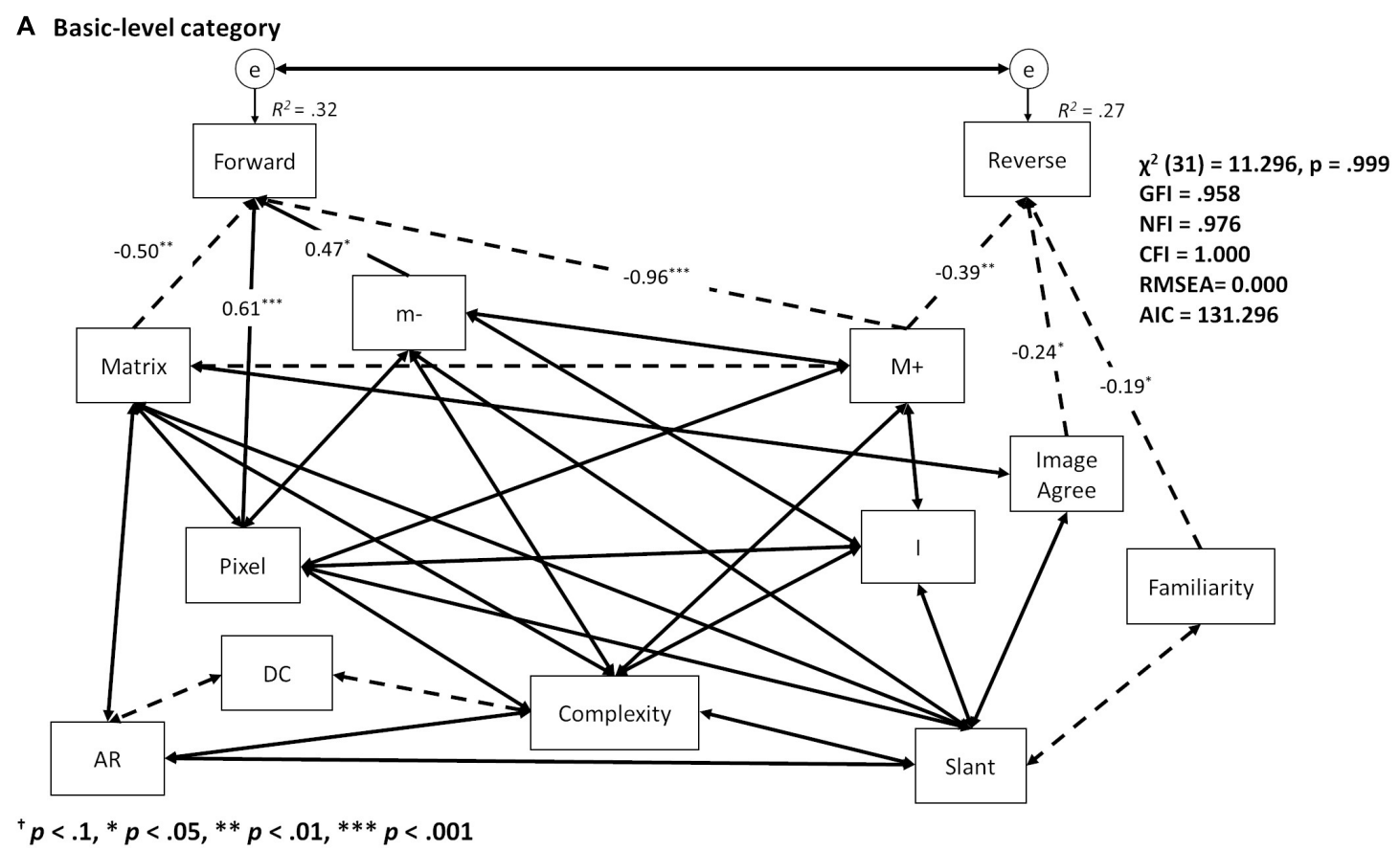

B Sueprordinate-level category

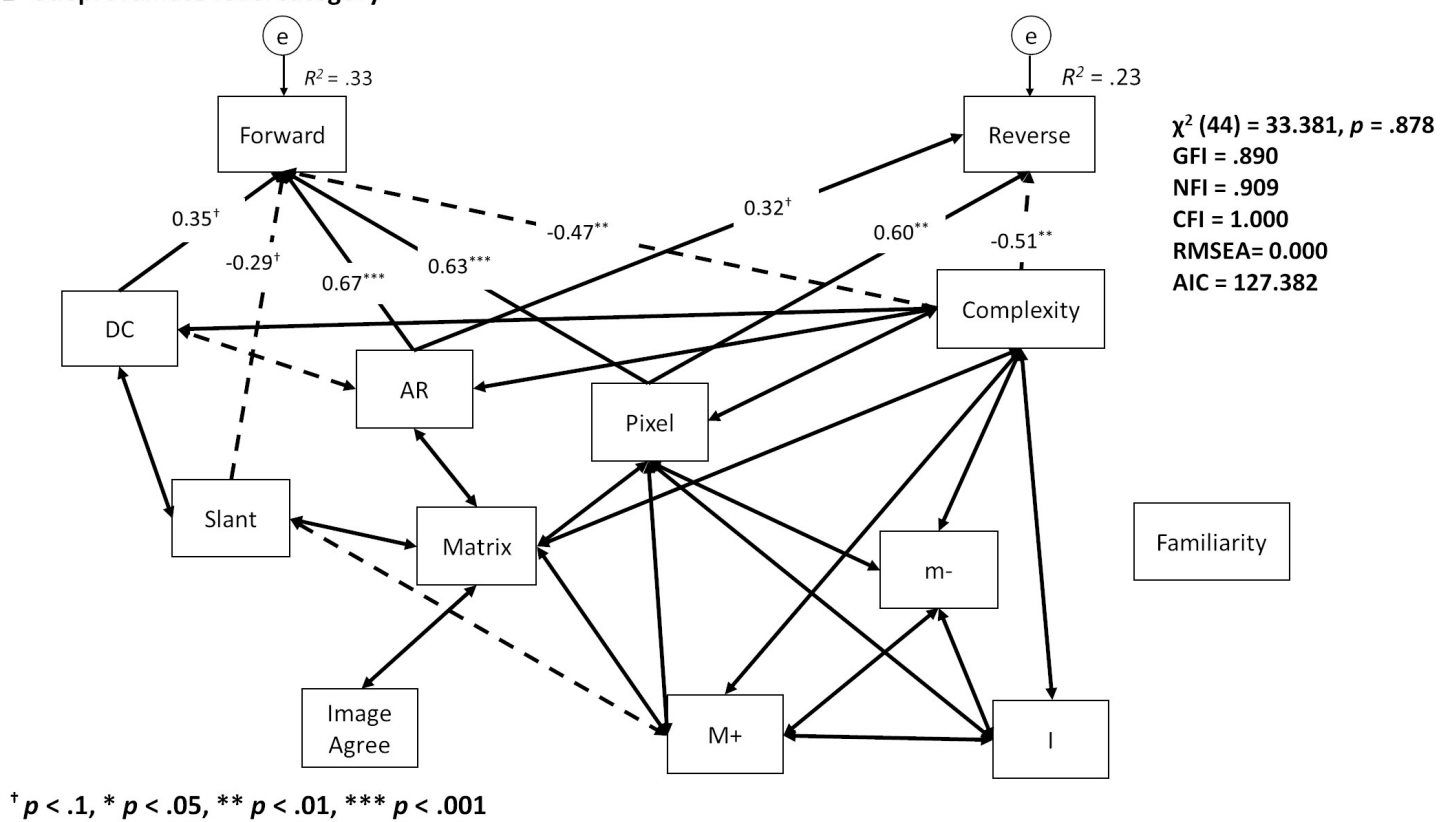

FIGURE 6 | Summary of the best fitting model in the path analysis of reaction time for the (A) basic and (B) superordinate levels. One-sided arrows indicate causal effects from starting to end variables; two-sided arrows show correlations between variables. Solid arrows indicate positive effects; dashed arrows indicate negative effects. Coefficients of correlation were abbreviated for better visibility.

while the effects of ImageAgree $(\beta=0.29, p<0.1)$ were marginally significant for the reverse condition (Figure 7A) $\left(\chi^{2}(47)=35.410, p=0.983\right.$, GFI $=0.879, \mathrm{NFI}=0.908$, $\mathrm{CFI}=1.000$, RMSEA $=0.000$, AIC $=123.410)$. Accuracy for the forward condition was influenced by Matrix $(\beta=0.43$, $p<0.01)$, Slant $(\beta=-0.58, \mathrm{p}<0.001)$, and ImageAgree $(\beta=-0.43, p<0.001)$ at the superordinate level, while accuracy for the reverse condition was influenced by Complexity $(\beta=0.55, p<0.001)$, Familiarity $(\beta=0.31, p<0.01)$, and ImageAgree $(\beta=-0.25, p<0.05)$ (Figure 7B) $\left(\chi^{2}\right.$ $(46)=34.400, p=0.896, \mathrm{GFI}=0.882, \mathrm{NFI}=0.910, \mathrm{CFI}=1.000$, RMSEA $=0.000$, AIC $=124.397$ ). 


\section{A Basic-level category}

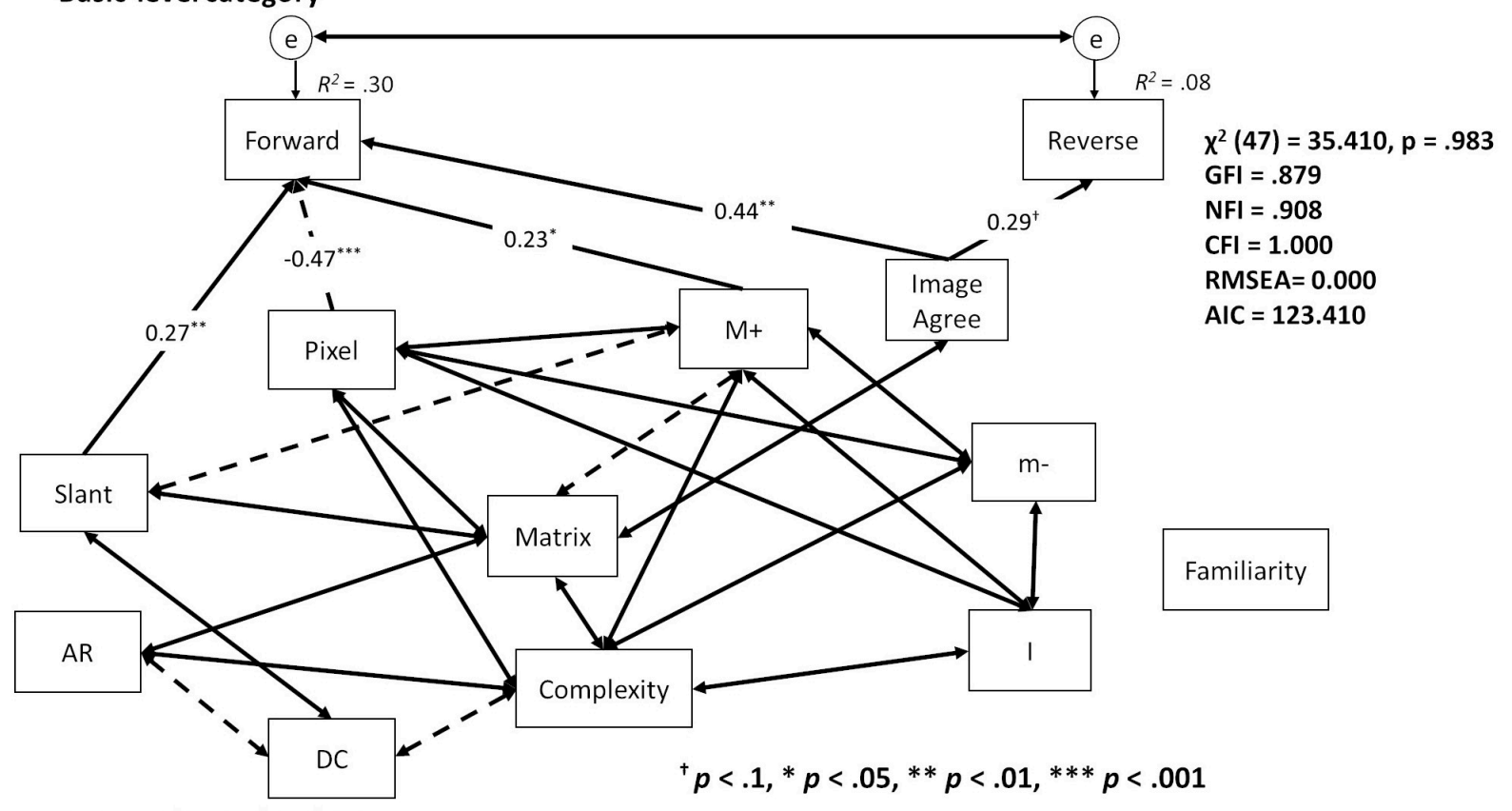

B Sueprordinate-level category

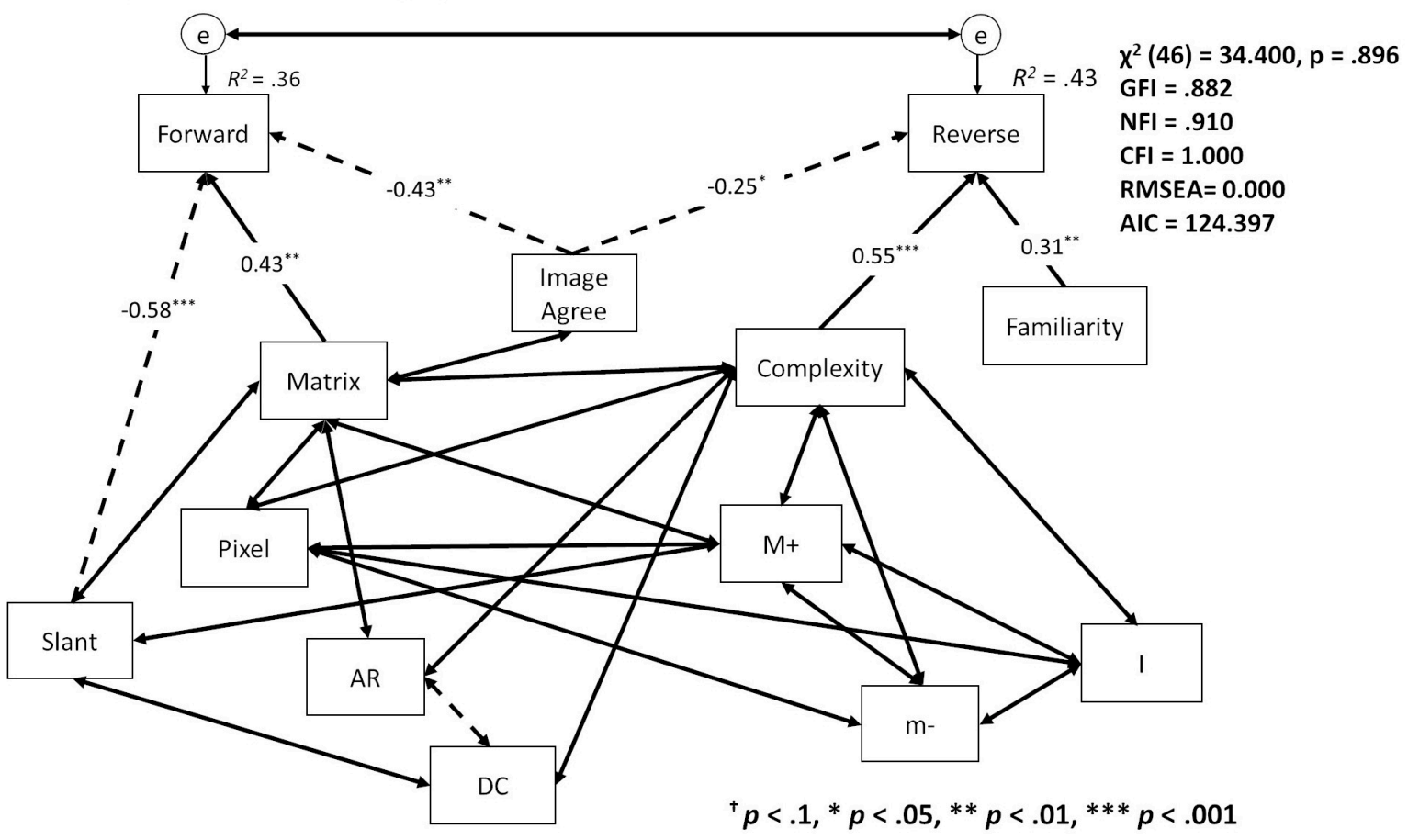

FIGURE 7 | Summary of the best fitting model in the path analysis of accuracy for the (A) basic and (B) superordinate levels. One-sided arrows indicate causal effects from starting to end variables; two-sided arrows show correlations between variables. Solid arrows indicate positive effects; dashed arrows indicate negative effects. Coefficients of correlation were abbreviated for better visibility.

\section{DISCUSSION}

This study investigated whether bottom-up or topdown processing was preferential depending on basic- or superordinate-level categorizations. We specifically controlled the position of target cues (i.e., forward and reverse condition) in the context of a categorization task. We also investigated which shape components affected basic- and superordinate-level categorizations by comparing dependence levels in regard to shape variables. Results indicated that the categorization process 
changed based on both the object category levels and target-word positions. For basic-level categorizations, the most important information for the forward condition included primary and local shape properties, while that for the reverse condition included the subjective estimation of line-drawing images. For superordinate-level categorizations, however, higher-level visual information (e.g., shape complexity) was highly important regardless of whether the condition was forward or reverse.

The results of an ANOVA conducted on reaction time indicated the presence of basic-level advantage effects for both the forward and reverse conditions, thus replicating the findings of Rosch et al. (1976). For accuracy, however, such effects were only found in the animal and plant categories. On the other hand, higher accuracy was detected for the superordinate-level furniture category when compared to that of the basic level. This may indicate that accuracy in the basic level depends on the variety of objects in a category. The basic level included a greater number of items for the animal and plant categories than for others (e.g., furniture and musical instruments). For example, the animal category contained a variety of candidates (e.g., dog, cat, and bird). As such, the names of basic-level animals and plants were more familiar to participants, who exhibited higher accuracy when compared to the superordinate level. The basiclevel furniture category, on the other hand, may have presented greater difficulty than other categories because constituent items contained many similar shapes (e.g., rectangles).

We also conducted a path analysis to determine the shape properties used in both the basic- and superordinate-level categorizations. Shape variables affected reaction time differently for the forward and reverse conditions only during basic-level categorizations, while the forward condition was influenced by the basic information found in line-drawings (e.g., Pixels and Matrix as well as curvature singularities such as $\mathrm{M}+$ and $\mathrm{m}-$ ). This indicated that basic-level categorizations depended on simple shape characteristics when participants matched items with stored representations during top-down processing. Further, the reverse condition was influenced by both the subjective rates of Familiarity and ImageAgree and the curvature singularities of $\mathrm{M}+$. For basic-level categorizations, this may indicate that bottom-up processing is dependent on subjective ratings in terms of object-representation accessibility. Thus, shape information was prioritized differently according to "target-word position" - and, thus, mode of processing - during basic-level categorizations.

For superordinate-level categorizations, the forward and reverse conditions shared similar reaction time effects in regard to the shape variables of Pixels, AR, and Complexity. This indicates that superordinate-level categorization depends on higher-level visual information related to Complexity for both bottom-up and top-down processing.

The path analysis of accuracy indicated different dependencies on shape variables during both basic- and superordinate-level categorizations for both the forward and reverse conditions. Categorizations in the forward condition depended on basic image properties (e.g., Pixels, Matrix, and Slant), indicating that forward condition categorizations arose from shape matching and simple shape properties from stimulus images, while reverse condition categorizations arose from subjective image ratings (e.g., Complexity, Familiarity, and ImageAgree). This indicates that, for the processing of superordinate-level categorizations in the forward condition, it is more important to match simple shape properties with stored object representations, whereas for the processing of superordinate-level categorization in the reverse condition, it is more important to match the shapes stored in object representations with received visual information.

The concept of different category levels is one of the most important in object recognition. The theory of object recognition indicates that the accessibility of object representations causes different response speeds and levels of accuracy between category levels. However, this study found that such categorizations were related to different shape properties for basic and superordinate levels. We conducted experiments that were specifically designed to control the sequence of target-word positions (i.e., forward and reverse conditions), and compared visual information dependence at both levels. For basiclevel categorizations, visual information differed between the forward and reverse conditions, indicating that different processes of object categorization change the required visual information through the received information. This processing difference may allow basic-level categorizations to operate in various situations both quickly and accurately. On the other hand, for superordinate-level categorizations, visual properties related to shape complexity had significant effects on reaction time and accuracy, regardless of forward or reverse conditions. As such, superordinate-level categorizations resulted in consistent responses even with minimal amounts of received information (i.e., during ultra-rapid categorizations; Thorpe et al., 1996).

In this study, we constructed a general model of object categorization and used some cross-category properties as indexes, such as a degree of circularities and curvature extrema. It is, however, possible that the indexes representing specific category properties may be involved in contribute to a more suitable model (see also, Zhang et al., 2011, 2014). Indeed, some studies have shown that categorization performance is influenced by whether an object is living or non-living (McMullen and Purdy, 2006; Riddoch et al., 2008; Mahon et al., 2009; Praß et al., 2013). Furthermore, the effect of shape properties can change based on the viewpoint of an observer (Blanz et al., 1999; Zhang et al., 2018, 2019, 2020). These issues should be investigated through further research.

Daily behavior is influenced by object recognition. This study showed that object recognition is variable, or flexible in different situations (e.g., different category levels and order of received visual information). As such, the object recognition process may change based on individual behaviors. In other words, a flexible structure was constructed during object recognition to deal with various behaviors. A previous study showed that both children and adults with ASD failed to make quick and/or accurate categorizations when compared with typically developing participants (Gastgeb et al., 2006; Gastgeb and Strauss, 2012). This may indicate that individuals 
with ASD have less flexibility during object recognition when compared to neurotypical individuals, thus causing difficulties in daily life. As such, additional research is necessary to clarify the relationship between object-recognition flexibility and daily behavior.

In sum, this study investigated whether categorization decisions, at both the basic and superordinate levels, depended on bottom-up and/or top-down processing according to the forward condition (presenting the target name before the linedrawing stimulus) and reverse condition (presenting the target name after the line-drawing stimulus). Further, it evaluated what visual information is required for quick and accurate categorization at each level. The results suggested that the categorization process changed based on both the object category level and target-word position. For basic-level categorizations, primary and local shape properties were important for the forward condition, while subjective estimation of line drawing images was important for the reverse condition. Superordinatelevel categorizations depended on higher-level visual information (e.g., shape complexity) regardless of whether the condition was forward or reverse.

\section{DATA AVAILABILITY STATEMENT}

The raw data supporting the conclusions of this article will be made available by the authors, without undue reservation, to any qualified researcher.

\section{REFERENCES}

Attneave, F. (1954). Some informational aspects of visual perception. Psychol. Rev. 61, 183-193. doi: 10.1037/h0054663

Biederman, I. (1987). Recognition-by-components: a theory of human image understanding. Psychol. Rev. 94, 115-147. doi: 10.1037/0033-295X.94. 2.115

Blanz, V., Tarr, M. J., and Bülthoff, H. H. (1999). What object attributes determine canonical views? Perception 28, 575-599. doi: 10.1068/p2897

De Winter, J., and Wagemans, J. (2008). The awakening of attneave's sleeping cat: identification of everyday objects on the basis of straightline versions of outlines. Perception 37, 245-270. doi: 10.1068/ p5429

Driver, J., and Baylis, G. C. (1996). Edge-assignment and figure-ground segmentation in short-term visual matching. Cogn. Psychol. 31, 248-306. doi: 10.1006/cogp.1996.0018

Gastgeb, H. Z., and Strauss, M. S. (2012). Categorization in ASD: the role of typicality and development. Perspect. Lang. Learn. Educ. 19, 66-74. doi: 10. 1044/lle19.2.66

Gastgeb, H. Z., Strauss, M. S., and Minshew, N. J. (2006). Do individuals with autism process categories differently? The effect of typicality and development. Child Dev. 77, 1717-1729. doi: 10.1111/j.1467-8624.2006. 00969.x

Grill-Spector, K., and Kanwisher, N. (2005). Visual recognition: as soon as you know it is there, you know what it is. Psychol. Sci. 16, 152-160. doi: 10.1111/ j.0956-7976.2005.00796.x

Johnson, J. S., and Olshausen, B. A. (2005). The earliest EEG signatures of object recognition in a cued-target task are postsensory. J. Vis. 5, 299-312. doi: 10. $1167 / 5.4 .2$

Johnson, K. E., and Mervis, C. B. (1997). Effects of varying levels of expertise on the basic level of categorization. J. Exp. Psychol. Gen. 126, 248-277. doi: 10.1037/0096-3445.126.3.248

\section{ETHICS STATEMENT}

The studies involving human participants were reviewed and approved by the Ethical Committee of the Doshisha University. The patients/participants provided their written informed consent to participate in this study.

\section{AUTHOR CONTRIBUTIONS}

KT designed the study, conducted the experiments, and analyzed the results. KT and KK wrote the original draft of the manuscript. YT and SI supervised the project. All authors revised, read, and approved the submitted version.

\section{FUNDING}

This study was supported in part by the Japan Society for the Promotion of Science's (JSPS) Grants-in-Aid for Scientific Research (KAKENHI) (Grant Number: 16KT0002) to YT and the project for the improvement of the Joint Research Center for Baby Science.

\section{ACKNOWLEDGMENTS}

We would like to thank Editage (www.editage.com) for English language editing.

Jolicoeur, P., Gluck, M. A., and Kosslyn, S. M. (1984). Pictures and names: making the connection. Cogn. Psychol. 16, 243-275. doi: 10.1016/0010-0285(84) 90009-4

Kennedy, J. M., and Domander, R. (1985). Shape and contour: the points of maximum change are least useful for recognition. Perception 14, 367-370. doi: $10.1068 / \mathrm{p} 140367$

Koenderink, J. J., and van Doorn, A. J. (1982). The shape of smooth objects and the way contours end. Perception 11, 129-137. doi: 10.1068/p110129

Macé, M. J.-M., Joubert, O. R., Nespoulous, J.-L., and Fabre-Thorpe, M. (2009). The time-course of visual categorizations: you spot the animal faster than the bird. PLoS One 4:e5927. doi: 10.1371/journal.pone.0005927

Mack, M. L., and Palmeri, T. J. (2011). The timing of visual object categorization. Front. Psychol. 2:165. doi: 10.3389/fpsyg.2011.00165

Mahon, B. Z., Anzellotti, S., Schwarzbach, J., Zampini, M., and Caramazza, A. (2009). Category-specific organization in the human brain does not require visual experience. Neuron 63, 397-405. doi: 10.1016/j.neuron.2009.07.012

Marr, D. (1982). Vision: A Computational Investigation Into the Human Representation and Processing of Visual Information. New York, NY: W. H. Freeman and Company.

McMullen, P. A., and Purdy, K. S. (2006). Category-specific effects on the identification of non-manipulable objects. Brain Cogn. 62, 228-240. doi: 10. 1016/j.bandc.2006.06.002

Nakayama, K., He, Z. J., and Shimojo, S. (1995). "Visual surface representation: a critical link between lower-level and higher-level vision," in An Invitation to Cognitive Science: Visual Cognition. Vol. 2, eds S. M. Kosslyn, and D. N. Osherson (Cambridge, MA: MIT Press), 1-70.

Norman, J. F., Phillips, F., and Ross, H. E. (2001). Information concentration along the boundary contours of naturally shaped solid objects. Perception 30, 1285-1294. doi: 10.1068/p3272

Praß, M., Grimsen, C., König, M., and Fahle, M. (2013). Ultra rapid object categorization: effects of level. Animacy and context. PLoS One 8:e68051. doi: 10.1371/journal.pone.0068051 
Riddoch, M. J., Humphreys, G. W., Akhtar, N., Allen, H., Bracewell, R. M., and Schofield, A. J. (2008). A tale of two agnosias: distinctions between form and integrative agnosia. Cogn. Neuropsychol. 25, 56-92. doi: 10.1080/ 02643290701848901

Rogers, T. T., and Patterson, K. (2007). Object categorization: reversals and explanations of the basic-level advantage. J. Exp. Psychol. Gen. 136, 451-469. doi: 10.1037/0096-3445.136.3.451

Rosch, E., Mervis, C. B., Gray, W. D., Johnson, D. M., and Boyes-Braem, P. (1976). Basic objects in natural categories. Cogn. Psychol. 8, 382-439. doi: 10.1016/ 0010-0285(76)90013-X

Rubin, E. (1958). "Figure and ground," in Readings in Perception, eds D. C. Beardslee, and M. Wertheimer (New York, NY: David Van Nostrand), 194-203.

Snodgrass, J. G., and Vanderwart, M. (1980). A standardized set of 260 pictures: norms for name agreement, image agreement, familiarity, and visual complexity. J. Exp. Psychol. Hum. Learn. Mem. 6, 174-215. doi: 10.1037/02787393.6.2.174

Tanaka, J. W., and Taylor, M. (1991). Object categories and expertise: is the basic level in the eye of the beholder? Cogn. Psychol. 23, 457-482. doi: 10.1016/00100285(91)90016- H

Taniguchi, K., Kuraguchi, K., and Konishi, Y. (2018). Task difficulty makes 'No' response different from 'Yes' response in detection of fragmented object contours. Perception 47, 943-965. doi: 10.1177/03010066187 87395

Thorpe, S., Fize, D., and Marlot, C. (1996). Speed of processing in the human visual system. Nature 381, 520-522. doi: 10.1038/381 $520 \mathrm{a} 0$

Ullman, S. (1996). High-Level Vision: Object Recognition and Visual Cognition. Cambridge, MA: MIT Press.

Zhang, C., Cheng, J., and Tian, Q. (2018). Multiview label sharing for visual representations and classifications. IEEE Trans. Multimed. 20, 903-913. doi: 10.1109/TMM.2017.2759500
Zhang, C., Cheng, J., and Tian, Q. (2019). Multiview, few-labeled object categorization by predicting labels with view consistency. IEEE Trans. Cybern. 49, 3834-3843. doi: 10.1109/TCYB.2018.2845912

Zhang, C., Cheng, J., and Tian, Q. (2020). Multi-view image classification with visual, semantic and view consistency. IEEE Trans. Image Process 29, 617-627. doi: 10.1109/TIP.2019.2934576

Zhang, C., Liang, C., Li, L., Liu, J., Huang, Q., and Tian, Q. (2017). Fine-grained image classification via low-rank sparse coding with general and class-specific codebooks. IEEE Trans. Neural Netw. Learn. Syst. 28, 1550-1559. doi: 10.1109/ TNNLS.2016.2545112

Zhang, C., Liu, J., Liang, C., Xue, Z., Pang, J., and Huang, Q. (2014). Image classification by non-negative sparse coding, correlation constrained low-rank and sparse decomposition. Comput. Vis. Image Underst. 123, 14-22. doi: 10. 1016/j.cviu.2014.02.013

Zhang, C., Liu, J., Tian, Q., Xu, C., Lu, H., and Ma, S. (2011). "Image classification by non-negative sparse coding, low-rank and sparse decomposition," in Proceedings / CVPR, IEEE Computer Society Conference on Computer Vision and Pattern Recognition. IEEE Computer Society Conference on Computer Vision and Pattern Recognition. CVPR 2011 (Providence, RI: Institute of Electrical and Electronics Engineers), 1673-1680. doi: 10.1109/CVPR.2011.5995484

Conflict of Interest: The authors declare that the research was conducted in the absence of any commercial or financial relationships that could be construed as a potential conflict of interest.

Copyright () 2020 Taniguchi, Kuraguchi, Takano and Itakura. This is an open-access article distributed under the terms of the Creative Commons Attribution License (CC BY). The use, distribution or reproduction in other forums is permitted, provided the original author(s) and the copyright owner(s) are credited and that the original publication in this journal is cited, in accordance with accepted academic practice. No use, distribution or reproduction is permitted which does not comply with these terms. 


\section{OPEN ACCESS}

Edited by: Ilaria Grazzani,

University of Milano-Bicocca, Italy

Reviewed by:

Flavia Lecciso,

University of Salento, Italy

Antonella D'Amico,

University of Palermo, Italy

${ }^{*}$ Correspondence:

Cinzia Di Dio

cinzia.didio@unicatt.it

Specialty section:

This article was submitted to

Developmental Psychology, a section of the journal

Frontiers in Psychology

Received: 20 December 2019

Accepted: 28 February 2020

Published: 03 April 2020

Citation:

Di Dio C, Manzi F. Peretti G, Cangelosi A, Harris PL, Massaro D and Marchetti A (2020) Shall I Trust You? From Child-Robot Interaction

to Trusting Relationships.

Front. Psychol. 11:469.

doi: 10.3389/fpsyg.2020.00469

\section{Shall I Trust You? From Child-Robot Interaction to Trusting Relationships}

\author{
Cinzia Di Dio ${ }^{1 *}$, Federico Manzi ${ }^{1}$, Giulia Peretti ${ }^{1}$, Angelo Cangelosi ${ }^{2}$, Paul L. Harris ${ }^{3}$, \\ Davide Massaro ${ }^{1}$ and Antonella Marchetti ${ }^{1}$
}

${ }^{1}$ Research Unit on Theory of Mind, Department of Psychology, Università Cattolica del Sacro Cuore, Milan, Italy, ${ }^{2}$ School of Computer Science, The University of Manchester, Manchester, United Kingdom, ${ }^{3}$ Graduate School of Education, Harvard University, Cambridge, MA, United States

Studying trust in the context of human-robot interaction is of great importance given the increasing relevance and presence of robotic agents in the social sphere, including educational and clinical. We investigated the acquisition, loss, and restoration of trust when preschool and school-age children played with either a human or a humanoid robot in vivo. The relationship between trust and the representation of the quality of attachment relationships, Theory of Mind, and executive function skills was also investigated. Additionally, to outline children's beliefs about the mental competencies of the robot, we further evaluated the attribution of mental states to the interactive agent. In general, no substantial differences were found in children's trust in the play partner as a function of agency (human or robot). Nevertheless, 3-year-olds showed a trend toward trusting the human more than the robot, as opposed to 7-year-olds, who displayed the reverse pattern. These findings align with results showing that, for 3- and 7-yearolds, the cognitive ability to switch was significantly associated with trust restoration in the human and the robot, respectively. Additionally, supporting previous findings, we found a dichotomy between attributions of mental states to the human and robot and children's behavior: while attributing to the robot significantly lower mental states than the human, in the Trusting Game, children behaved in a similar way when they related to the human and the robot. Altogether, the results of this study highlight that similar psychological mechanisms are at play when children are to establish a novel trustful relationship with a human and robot partner. Furthermore, the findings shed light on the interplay - during development - between children's quality of attachment relationships and the development of a Theory of Mind, which act differently on trust dynamics as a function of the children's age as well as the interactive partner's nature (human vs. robot).

Keywords: developmental robotics, HRI, Theory of Mind, attachment, social interaction

\section{INTRODUCTION}

One of the challenges of contemporary robotics is long-term interaction, which assumes that competent robot partners will have many human-like characteristics, enabling the complexity and multidimensionality of human interactions. This objective has been strengthened by a new interdisciplinary approach to robotics, i.e. Developmental Robotics (Cangelosi and Schlesinger, 2015). For example, Vinanzi et al. (2019) have proposed an artificial cognitive architecture to 
simulate human decision making in the robot by using concepts from developmental theories, such as Theory of Mind (ToM). From this perspective, the implementation of an artificial architecture, together with an understanding of the human's response to the behavior of a robot within a relational context, aims to shed light on the processes involved in establishing a relationship with robotic agents (e.g. Wykowska et al., 2016; Wiese et al., 2017). Within this framework, trust comes into play as a key psychological component underpinning successful interpersonal relationships, particularly when these include at least one robotic agent. In the present study, we observed children between the ages of 3 and 9 who established relationships of trust with a human or the humanoid robot NAO in a simple "guessing" game in which the child and the human or robot played together. Furthermore, not only did we assess trust acquisition, but also a key feature of real-life relational dynamics: trust restoration after trust loss. As a matter of fact, trust is a dynamic process based on past relational experiences and, as such, it is subject to fluctuations operationalized in this study via three phases of trust: acquisition, loss, and restoration. The latter phase is of particular interest. While human forgiveness has been studied in different conditions (see, for example, Grover et al., 2019), the investigation of how relational failures may affect trust restoration in a relationship with a robot is still unexplored.

In psychology, trust can be described as "a multidimensional psychological attitude involving beliefs and expectations about the reliability of the trustee resulting from social experiences involving uncertainty and risk" (Jones and George, 1998; in Lewis et al., 2018, p. 137). Trust in the choices of unknown people can be envisaged also in situations where we passively witness their behavior, with consequences on our own decisions (e.g. Rizzato et al., 2016). The multidimensional nature of trust encompasses the idea that trust can be built based on either (or both) objective factors or (and) an emotional, quite irrational, attitude toward the partner (Lahno, 2001). In this light, emotional trust can be conceived as somewhat independent of objective information. In this study, we recreated a situation of total uncertainty in which the choices of a partner, who should be trusted, are not based on the evaluation of objective elements, and also the decision of the child to trust in the partner are devoid of rational elements. Rather, the decision to trust or not to trust the partner's choices is consequentialist in nature considering that, until proven otherwise, the partner is always accurate in her/his/its choices. That is, trust is progressively built through constant endorsement of the play partners' reliability in providing correct responses (see, Rotenberg, 2010). From this perspective, conformation to the other's choices reflected levels of trust acquisition as well as acceptance of the other as a potential partner (Nass et al., 1995; Nass and Moon, 2000).

The establishment of trusting relationships is critical for effective interpersonal dynamics. This is particularly relevant where children are called to build new relationships with peers, educators, and other adults. An example of the importance of the construction of interpersonal trust is highlighted in a study with children under protection services (Petrocchi et al., 2018). In these critical circumstances, not only does the success of the social interventions rely on building trusting relationships between the child's parents and the social workers, but also between the latter and the child in need of psychosocial adjustment (HaffordLetchfield and Spatcher, 2007). Developmental research on the construction of trusting relationships shows that trust dynamis change significantly as a function of age. For example, children aged 3 years tend to display trust if the informant is consistently 182 accurate (Clément et al., 2004; Koenig et al., 2004; Pasquini et al., 2007) but are relatively unforgiving in case of mistakes (Harris, 2007), effectively showing a certain behavioral rigidity. With development, particularly from 4 years of age, children become more flexible: they do not rely on another's testimony in an indiscriminate fashion (Harris, 2007) and show selective trust in others' testimony (Clément et al., 2004; Chan and Tardif, 2013). They attend both to the information available at that moment, and to the reliability that a person has shown in the past.

Human trusting relationships are also shaped by past relational histories, originating with primary caregivers (e.g. Camisasca et al., 2017; Giovanelli et al., 2020; Marchetti et al., 2020) and extending to subsequent, significant affective relationships (Bowlby, 1969, 1973, 1980). It has been suggested that children's decision to place trust in an unknown informant, especially in a context of uncertainty, may also depend on generalizing from their personal attachment history (Fonagy, 1998; Allison and Fonagy, 2016; Fonagy et al., 2019; see also, Bo et al., 2017; Luo et al., 2018). For example, securely attached children are more flexible in establishing trustful relationships with epistemically reliable strangers than children with a fragile relational past (see, for example, Corriveau et al., 2009). In this view, we may ask about interactions that involve partners with whom there is no affective history and with whom a relationship needs to be built on the basis of novel interactional dynamics that develop hic et nunc.

Likewise, the development of the individual's cognitive competencies is important, particularly for the definition of the informant's epistemic reliability. Cognitive skills allow individuals to reason about the other's perspective and to objectively evaluate informational access. In this respect, the development of a ToM enabling individuals to conceptualize the mental states that guide behavior (Wimmer and Perner, 1983) and social competence (Premack and Woodruff, 1978; Perner and Wimmer, 1985; see also, Lombardi et al., 2018; for a review, see Wellman et al., 2001) is a necessary prerequisite for the establishment of trusting relationships (Fusaro and Harris, 2008; Lecciso et al., 2011; Sharp et al., 2011; Lucas et al., 2013; Brosseau-Liard et al., 2015; Rotenberg et al., 2015; Van Reet, 2015). The association between the establishment of trust and the development of ToM competencies was first hypothesized by Koenig and Harris (2005) who found that only 4-year-olds, and not 3-year-olds, showed selective trust toward a previously accurate informant. More recently, associating trust beliefs with ToM abilities in children aged 9 years, Rotenberg et al. (2015) further showed that children's trust beliefs in others are associated with both second-order false belief ToM ability as well as with advanced ToM abilities (see also Van Reet et al., 2015). As well-documented (e.g. Carlson and Moses, 2001; Frye et al., 1995), there is 
a strict relationship between false belief understanding and more general executive function skills. One may then question about the overlap between these competencies in building trust. Still, socio-cognitive skills mediated by one's ability to understand the others' knowledge, like false belief, appear to be more influential in building selective trust rather than more general executive function skills, at least in some cultures (Lucas et al., 2013).

In relation to human-robot interaction, studies that have specifically investigated trust in a robot agent or system have typically involved adult participants. These studies have either used explicit measures of trust assessment, mostly involving selfreports (e.g. Yagoda and Gillan, 2012), or implicit measures of trust assessment. Explicit measures of trust are strongly subject to the idiosyncratic attitude and the impression that one has of the robot, which are often based on beliefs and not on actual interactional experiences with the robot; on the other hand, implicit measures of trust generally involve the postulation of hypotheses framed by specific environmental and theoretical conditions that are then tested during actual interaction with a robotic system. Gaudiello et al. (2016), for example, investigated the role played by functional acceptance (perceived ease of use, usefulness) and social acceptance (generally linked to social competencies) of the robot iCub for effective human-robot interaction. These two aspects appear to be most consistently associated with an enduring perception of the robot's skills, i.e. its usefulness and sociality (ShawGarlock, 2009; Heerink, 2010). As a most comprehensive measure of functional and social acceptance of the robot, the users' trust in the robot was assessed as a function of the robot's social and functional knowledge. The users' trust in the robot prevalently relied on its functional rather than social knowledge, although data generally highlighted adults' poor acceptance of, and a predominant distrust in robots. With children, the factors underpinning child human-robot interaction have not been systematically explored. There are several studies that inform about ways in which children interact, play, and learn from a robotic agent in school and educational contexts (Kanda et al., 2004; Okumura et al., 2013a,b; Breazeal et al., 2016; Baxter et al., 2017; Belpaeme et al., 2018; Cangelosi and Schlesinger, 2018; Di Dio et al., 2019). These studies have shown that children tend to interact with robot partners in a humanlike manner, proving to be sensitive to verbal and non-verbal signals, such as eye gaze (Okumura et al., 2013a,b), and often attributing mentalistic competencies to the robot (for a review, see Marchetti et al., 2018). In this respect, the work by Short et al. (2010) shows that unfair/cheating robots in a common "rock-paper-scissors" child-game are able to elicit interest in the child as well as a greater tendency to attribute intentions to the robot. This study brings further support to the idea that human-like behavior (either trustful or even deceptive) is associated to a greater interactional potential toward a robot partner.

In the present study, trust was explored through a novel Trusting Game (TG) named "Guess where it is" requiring the interactive partner (either the human or the robot) and, subsequently, the child to guess the position of a doll hidden under a box. Through the structure of the game, we set the conditions for the child to consequentially make the same decisions as the play partner, thus ultimately establishing a trusting relationship (e.g. Nass and Moon, 2000): the other becomes trustworthy because it demonstrates that her/his/its choices, even if random, always lead to a correct answer. This procedure benefitted from having the child gradually build trust in the partner during a social interaction. It was chosen not to establish epistemic trust before the game following best known procedures (see, for example, Koenig and Harris, 2005; Corriveau and Harris, 2009) because we also wanted to appreciate the dynamics of trust construction when interacting with different relational agents, i.e. the human and the robot. Once trust had been acquired, as indexed by a consistent agreement between the play partner's and the child's responses, the phases of loss of trust and trust restoration put the child's trust to test. These latter phases were most critical for the child because s/he had to reconsider the newly established trust in the robot or the human. To better understand what psychological factors are in place when building a trusting relationship with the robot, as compared to the human, we addressed specific different chronological ages (e.g. Lombardi et al., 2017) where the development of affective and cognitive processes may be distinctively influential on trust. Also, to better appreciate how trust is configured within robot-human and human-human interaction, we avoided creating competitive or collaborative conditions that could have polarized the dynamics of trust-building. As a matter of fact, the type of interaction can significantly influence trust (Hancock et al., 2011) by negatively or positively skewing trust in case of competition or collaboration, respectively (Kidd, 2003; Kuchenbrandt and Eyssel, 2012). Therefore, we had the children play for the mere fun of playing with a little thank-you gift delivered at the end of the game (the structure of the TG is detailed in section "Materials and Methods"). Finally, we further assessed the distinctive contribution of ToM and executive function skills in building trust at different developmental ages, thus extending current literature by also exploring these cognitive components when children interacted with a robot or a human agent.

To make the child perceive the robotic agent NAO as a real interactional partner, it was introduced to children in a preliminary session when they were familiarized with some of the robot's physical and social skills (walking, moving its arms, talking, greeting, etc.) (see Vogt et al., 2017). To make its behavior human-like, when playing its turn during the TG, NAO used simple and clear verbal indications, accompanied by gestures indicating the possible target position of the doll. Additionally, the robot was programmed to alternate between looking at the play setup and the child, reproducing a realistic attentional shift (Zanatto et al., 2019).

The children's perception of the robot's mental qualities as compared to the human was evaluated through the Attribution of Mental States (AMS) questionnaire (Di Dio et al., 2018, 2019). This measure has consistently shown that school-age children do discriminate between the human and the robot in mental terms, although, during interaction, children also typically display 
similar behaviors toward both. Accordingly, we hypothesized to find substantial differences in the children's attribution of mental states to the human and the robot, whereas a similar trustbuilding dynamics when interacting with either partner during the TG. Additionally, we hypothesized to find a greater tendency to trust, especially in the human, among younger children whose trust is possibly mainly driven by affect rather than cognition. On the other hand, we hypothesized to find the establishment of more reflective trusting relationships among children given the development of ToM competencies. No specific predictions were advanced with respect to the role of executive functions in trust dynamics given the fair lack of specific evidence in this respect.

\section{MATERIALS AND METHODS}

\section{Participants}

Ninety-four (94) Italian kindergarten and school-age children participated in the experiment. The children were divided into four age groups as follows: 3-year-olds ( $N=22,9$ females), 5year-olds ( $N=24,13$ females $), 7$-year-olds ( $N=25,13$ females), and 9 -year-olds ( $N=23,12$ females). The children were recruited from a preschool and a primary school of Milan. The children's parents received a written explanation of the procedure of the study, the measurement items, and the materials used, and they gave written consent. Children were not identified by parents or teachers for learning and/or socio-relational difficulties. The study was approved by the Local Ethics Committee (Università Cattolica del Sacro Cuore, Milan).

\section{Tasks}

The children were assessed in two experimental sessions on different days within a 2 -week time frame. In the first session, the children were administered the following tests: AMS scale (inspired by the work of Martini et al., 2016), TG task [inspired by the work of Yang et al. (2017)], and a first-order and (for 5- to 9-year-olds) a second-order False-Belief task (Wimmer and Perner, 1983; Perner and Wimmer, 1985). In the second session, the children were administered a further version of the first-order and second-order False-Belief task, the quality of attachment relationships (SAT) test (Liverta Sempio et al., 2001), an executive function task (Dimensional Change Card Sort, DCCS; Zelazo, 2006) for the 3- and 5-year-olds, and the Developmental NEuroPSYchological Assessment (NEPSY II; Korkman et al., 2007) subtest for the 7- and 9-year-olds. Both tests assess the ability to switch between responses.

\section{Attribution of Mental States}

The AMS scale is a measure of the mental states that participants attribute when looking at pictures depicting specific characters, in this case a human and the robot NAO. The scale is an ad hoc questionnaire that was based on Martini et al. (2016). AMS has been used in previous works (Di Dio et al., 2018, 2019; see also, Di Dio et al., 2020; Manzi et al., 2020) and has proven fairly consistent in outlining age-specific response patterns with respect to attribution of mental states to both robots and humans. Children were asked 25 questions grouped in five different state categories: Perceptive, Emotional, Desires and Intentions, Imaginative, and Epistemic. The child had to respond "Yes" or "No" to each question. If the answer was Yes, then the experimenter asked a follow-up question: "How much? A little bit or very much?", yielding a 3-point scale. For example, in answer to the question: "Do you think that he/she/it can understand?", the range of answers could be: No (0), Yes, a little bit (1), or Yes, very much (2). The total score was the sum of all answers (range $=0-50$ ); the five partial scores were the sum of the answers within each category (score range $=0-10)$.

\section{Trusting Game}

The TG was inspired by the work of Yang et al. (2017). The game requires the play partner (either the human or the robot) and the child guess the position of a doll hidden under a box. By its nature, the game is neither explicitly collaborative nor competitive since both players have to independently guess the position of the doll and correct guesses do not lead to any tangible reward. The TG involves two players (i.e. a child participant - playing with either the experimenter or the robot) and a game-master (i.e. a second experimenter). The game consists of presenting to the players two boxes and a little doll that are positioned on a table that looks very much like a coffee table and at which both players are seated. The game-master, who sits on the opposite side of the table, hides the doll under one of the two boxes without being seen by the two players (see Figure 1 for a depiction of the experimental setup). The game consists of guessing where the game-master has hidden the doll. The game starts with the experimenter explaining verbally to the players the rules, showing them an example of a sequence: "Now you and (the other partner's name) will play a game together called "guess where it is." I'll show you how it is played. Here are two boxes and a little doll. I will hide the doll under one of the two boxes, but you won't see where I hide it because I will put this paper board in front of you, like this." After positioning the board, the experimenter moves the boxes around and then removes the board, placing it on the side of the table. The experimenter then informs the partners that they have to guess where the doll has been hidden by pointing at one of the boxes. Next, without revealing the doll's location, the experimenter asks: "It is all clear?". If both partners answer positively, then the play started. All children understood the instructions the first time. The children were also informed that they would receive a packet of stickers at the end of the game to thank them for their participation. Once the game began, the experimenter told the child that the partner (referred to by her/his/its name) would always make the first guess. The position of the doll was established a priori to correctly instruct (or program, if NAO) the play partner's choice during each phase of the game.

The TG involves three independent phases. The first phase [Trusting Acquisition (TA)] aims to assess the participant's acquisition of trust in the other player by calculating how many trials elapse before the child follows the other player's guess. Trust is assumed when the child follows the other player's guess on three consecutive trials. After trust acquisition, the game switches into the second phase [Mistrust Acquisition (MA)], which assesses the participant's acquisition of mistrust in the 

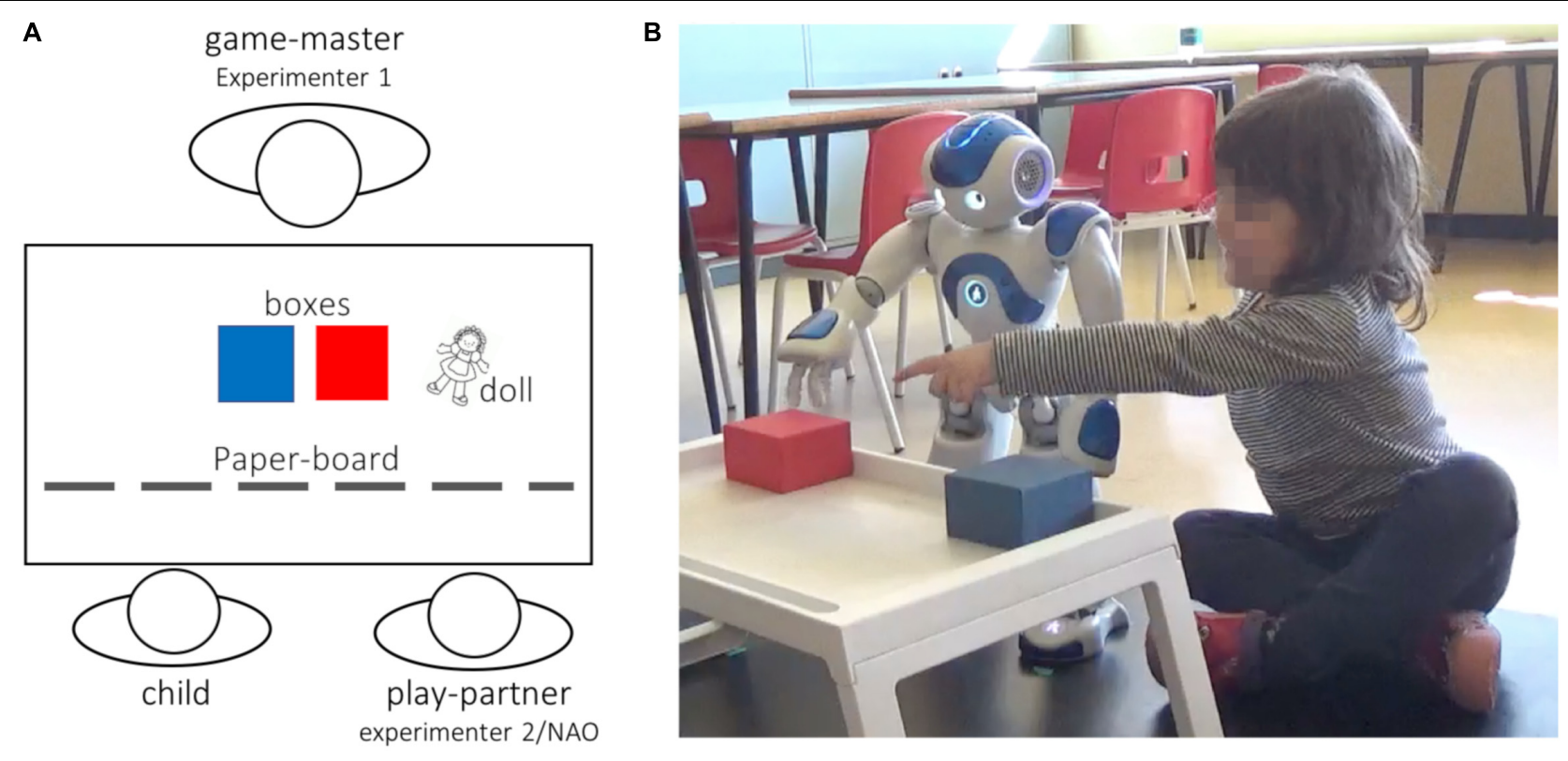

FIGURE 1 | Overview of the experimental setup in the Trust Game. (A) Overview of the participants' seating arrangement and stimuli. (B) Photograph representing the SoftBank Robotics NAO humanoid robot while playing the Trust Game with a child (subject).

other player by calculating how many trials it takes for the child not to follow the other player's guess. Mistrust is assumed when the child does not follow the other player's guess on three consecutive trials. The last phase [Trusting Restoration (TR)] shares the same play structure as the initial phase. The game lasted, on average, between 10 and $20 \mathrm{~min}$.

Each phase consisted of a maximum of 10 trials and ended after trust acquisition (phase 1), mistrust acquisition (phase 2), and trust restoration (phase 3 ). The switch to the following phase also occurred if the participant completed 10 trials within a given phase without completing the three-trial sequence. The dependent variable (DV) was the number of trials the child required before acquiring trust or mistrust. For example, in the initial phase, if the child started to follow the other player for three consecutive trials after the second trial (i.e. $\left.\begin{array}{llllllllll}0 & 0 & 1 & 1 & 1\end{array}\right)$, the participant scored 2 . If the child displayed trust immediately (i.e. 11 1), s/he scored 0 . If the child completed the 10 trials within each phase without acquiring trust or mistrust, she/he scored 8 , which is the maximum value that could possibly be attributed before ending the phase with a three-trial sequence. To compare data in the analyses, trust and trust restoration indexes were reversed to indicate, alongside trust loss, a comparable measure of the tendency to trust. Thus, a child could score between 0 (low trust) and 8 (high trust).

For the treatment of missing cases, we considered mean, median, and mode values, as well as children's most common response patterns. The median was ultimately chosen as the most representative index for replacing missing values. Accordingly, two children were recovered for age groups 3, 5, and 9 years; one child was recovered for the age group 7 years. When an entire session was missing, the values were not replaced and the child was removed from the analyses. Accordingly, one child was removed from age group 3 years and three children were removed from age group 7 years.

\section{Theory of Mind}

The Unexpected Transfer task (Wimmer and Perner, 1983) and the Unexpected Content task (Perner and Wimmer, 1985) were used to evaluate first-order ToM by assessing the acquisition of false beliefs understanding. First-order ToM entails a recursive thinking, which implies the meta-representation or the representation of a mental representation of a low complexity level, of the kind "I think that you think...". Children exhibit this competence at around 4 years of age with the emergence of false beliefs. The child is told a story involving two doll characters. One of the characters is deceived with respect to either the location or contents of an object and the child is tested for his/her ability to understand the character's false belief. For example, the unexpected transfer story is about two siblings playing with a ball in a room. One of the children puts the ball in a box and leaves the room. Meanwhile, the other child takes the ball out of the box, puts it in the basket and goes away. Finally, the first character comes back in the room and wants to play with the ball. At the end of the story, the experimenter asks the child the following questions: "What is the first place where she will look for the ball?"-referring to the first character (first-order false belief question); "Where did the child put the ball before going away?" (control memory question); "Where really is the ball?" (reality control question). The answers to the two control questions (memory and reality) were used to filter the children's performance. Having passed control questions, the test question about false belief is scored 1 if correct and 0 if incorrect. 
The development of a second-order false belief competence was assessed through the Ice-Cream Van task (Perner and Wimmer, 1985) and the Look-Prediction task (Liverta Sempio et al., 2001; Astington et al., 2002). Second-order ToM implies a meta-representation of a greater complexity with respect to first-order ToM, of the kind "I think that you think that s/he thinks...". Children aged from 7 years have typically matured this competence, although it can also emerge at an earlier age. The second-order ToM stories involve three characters presented on a storyboard. For example, the ice-cream van story is about Maria and Giovanni, who - while playing in the park - see an ice-cream van. Maria wants to buy an ice cream, but she has no money. She therefore decides to go home to take the money, sure that the ice-cream van will stay in the park. However, while Maria is away, Giovanni sees the ice-cream van moving away. Giovanni asks the ice-cream man where he is going, and the ice-cream man replies that he is going in front of the school to sell more ice creams. While Maria is leaving home, she sees the ice-cream man and she asks him where he is going. After knowing that he is moving to school, she says that now that she has the money, she can follow him to school. At the end of the story Giovanni goes to Maria's house, and asks her mother where her friend is. Maria's mum answers that Maria has just gone out to buy an ice cream. The child (participant) is then asked the following questions: "Where does Giovanni think Maria went to buy the ice cream? (second-order false belief); "Why does Giovanni think so?" (justification); "Does Maria know that the ice-cream van is in front of the school?" (first-order false belief); "Does Giovanni know that the ice-cream man spoke with Maria while she was leaving her house?" (reality control question); "Where did Maria go to buy the ice cream?" (memory control question). For both second-order false belief tasks, having passed the control questions, children scored 1 for correct statements and 0 for incorrect statements on both test and justification questions. A second-order false belief task total score was then calculated ranging from 0 (no response) to 2 (completely correct response) (Perner and Wimmer, 1985).

\section{Separation Anxiety Test-Family Version (F-SAT)}

The Separation Anxiety Test is a semi-projective task that evaluates the child's mental representation of his/her attachment to the caregiver. The original version developed by Hansburg (1972) for adolescents was adapted by Klagsbrun and Bowlby (1976) for children aged 4 to 7 years. In the latter version, six pictures are presented to the child, each depicting a situation of separation from a familiar caregiver. The child is asked to describe the protagonist's feelings, to justify them, and to predict what the protagonist will do, thereby probing the coping strategy of the protagonist. The Italian version used in this study (Liverta Sempio et al., 2001) is based on a modification of other versions of the same task (Fonagy et al., 1997).

The coding reflects three dimensions: (1) attachment, i.e. the ability to express vulnerability and need; (2) self-confidence, i.e. the ability to autonomously face separation; and (3) avoidance, i.e. the propensity to speak about the separation. Participants score 1 for each dimension. The final score is the result of the sum of the scores in the attachment scale and in the self-confidence scale, and of the sum of the inverse of the avoidance scale, calculated by subtracting this score from the total amount potentially obtainable on this scale. Scores range from 6 to 36, with higher scores reflecting greater quality of attachment relationship.

\section{Executive Function Skills}

Children aged 3 and 5 years were administered the DCCS assessing the capacity to switch responses [for a full description of the test, please refer to Zelazo (2006)]. Seven and 9-yearolds' executive functions were assessed using "A Developmental NEuroPSYchological Assessment” subtest (NEPSY II; Korkman et al., 2007), testing the ability to inhibit automatic responses and to switch between response types. The child looks at a series of black and white shapes or arrows and names either the shape or direction or makes an alternate response, depending on the color of the shape or arrow. In the present study, we used the combined scores of the Inhibition NEPSY-II subtest, which associates accuracy and speed of response. For a detailed description of the scoring criteria, please refer to the manual (Korkman et al., 2007).

\section{Experimental Procedure Introducing the Play Partners}

On a day that preceded the main experimental session, children were introduced to three play partners (two humans - a boy and a girl - and the robot) through video clips displayed in class on a large projector. In the videos, each of the potential partners said the same sentence: "Hello, my name is. I will be playing with you in the next days. See you soon. Bye." The videos represented the actors while exiting a room and waving their hand to say goodbye. In this way, the children saw that the robot NAO could walk, talk, and move its head and arms.

\section{Experimental Sessions}

The children were tested individually in a quiet room in their kindergarten or school. Tests were carried out by two researchers both in the morning and in the afternoon during normal activity. In the first session, the administration of the battery lasted approximately 20-30 min, depending on the child's age. The administration of the task in the second session took about 35$45 \mathrm{~min}$.

The first session started with the administration of AMS. The five AMS state categories (Perceptive, Emotional, Desires and Intentions, Imaginative, and Epistemic) were randomized across children. Afterward, children participated in the TG. At this point, the partner (i.e. human or robot) entered the experimental room and was introduced by the experimenter by his/her name: "Do you remember, this is ...". Then, both the child and the partner were invited to sit on the ground on a plastic carpet in front of an ad hoc table. The plastic carpet was used to correctly position NAO when children interacted with the robot. A paper black board was positioned next to the table, and was used to cover the setting when playing. A female experimenter played with girls and a male experimenter played with boys. Half of the children played with the robot in the first session and with 
the human in the second session. The other half underwent the reversed play order.

After the game, the child was administered one of the two first-order ToM tasks and, starting from 5 years of age upward, one of the two second-order ToM tasks. The order of the ToM tasks was randomized across children, so that those who performed, for example the unexpected transfer task in the first session, completed the unexpected content task in the second session. The same was true for the second-order ToM tasks. Finally, children were given two further assessments: SAT and executive function.

\section{Statistical Analysis}

Statistical analysis was carried out using the IBM Statistical Software Platform SPSS (v. 19.0). To evaluate possible differences in children's tendency to trust the human and robot partner as a function of the child's age and trust phase (acquisition, loss, and restoration), a repeated measures General Linear Model (GLM) analysis was carried out. The DV was the number of trials until children followed their partner (trust acquisition), stopped following their partner (trust loss), and again followed their partner (trust restoration) during the TG. To compare data from the three phases, trust and trust restoration indexes were reversed to indicate, together with trust loss, a comparable measure of the tendency to trust. That is, for all three phases of the TG, greater numbers correspond to a greater tendency to trust.

Additionally, correlation analyses (Pearson's $r$ ) were carried out to evaluate the relationship between the tendency to trust and (1) the quality of attachment relationships (SAT), (2) ToM (first- and second-order false beliefs tasks), and (3) executive function skills.

Finally, to assess possible differences in children's mental states attribution to the robot with respect to the human partner, a repeated measures GLM analysis comparing AMS scores between human and robot was carried out as a function of the children's age. For all the GLM analyses, the Greenhouse-Geisser correction was used for violations of Mauchly's Test of Sphericity, $P<0.05$. All post hoc comparisons were Bonferroni corrected.

\section{RESULTS}

\section{Trusting Game}

The GLM analysis, with three levels of phase (acquisition, loss, and restoration) and two levels of agency ( $\mathrm{HB}$ and RB) as within-subjects factors, and age group (four levels) as the between-subjects factor $(3,5,7$, and 9 years), was carried out to assess children's tendency to trust in the human and in the robot. An inspection of the box plots displaying the performance of each age group showed no extreme cases.

The results revealed a main effect of phase (Figure 2A), $F(2,172)=10.51, P<0.001$, partial $-\eta^{2}=0.11, \delta=0.99$, indicating that, independent of agency and age group, children exhibited a lower tendency to trust in phase 3 (trust restoration), compared to both phase 1 (trust acquisition), $M$ diff $=1.19$; SE, $0.26 ; P<0.001$, and phase 2 (trust loss), $M$ diff $=0.94$; SE, 0.27; $P<0.01$. Additionally, age-related differences were found, $F(3,86)=8.76, P<0.001$, partial $-\eta^{2}=0.23, \delta=0.99$. More specifically, 3-year-olds showed a greater tendency to trust than the other age groups including the 5-year-olds, $M$ diff $=1.83$; SE, $0.52 ; P<0.01 ; 7$-year-olds, $M$ diff $=1.1$; SE, $0.53 ; P<0.05$; and 9-year-olds, $M$ diff $=2.64$; SE, 0.53; $P<0.001$. No interactions were found between phase and age group, $P>0.05$. Additionally, agency did not have any impact as a main effect and in the interaction with the other variables, $P>0.05$.

Having found a consistent correlation across ages between first-order ToM and performance in the TG as described below, a further GLM was carried out using first-order ToM as a covariate. This analysis revealed a main effect of agency (Robot $\mathrm{RB}>$ Human Being - HB), $F(1,85)=4.99, P<0.05$, partial$\eta^{2}=0.06, \delta=0.60$, and a significant interaction of agency $\times$ age group, $F(3,172)=2.81, P<0.05$, partial $-\eta^{2}=0.09, \delta=0.66$. The post hoc analyses showed that while 3-year-olds tended to generally trust in the human more than in the robot, $M$ diff $=1.04$; SE, $0.67 ; P<0.05$, children aged 7 years tended to trust in the robot more than in the human, $M$ diff $=1.33$; SE, $0.56 ; P<0.05$. This interaction is plotted in Figure 2B.

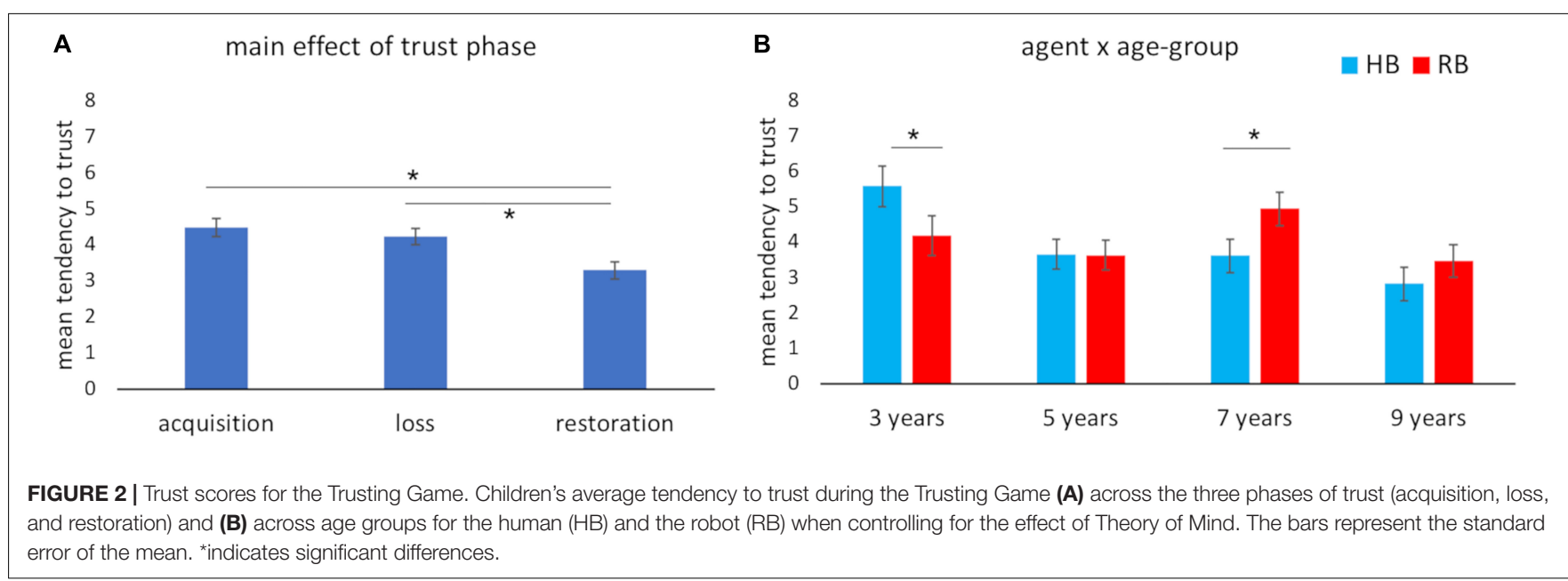




\section{Correlations}

\section{Trusting and SAT}

As shown in Table 1, most of the significant correlations between the quality of attachment relationships (SAT) and the tendency to trust were positive, i.e. more securely attached children showed a greater tendency to trust in the play partner's choice. These relationships were found mainly when children played with the human, and especially in the youngest age group. For 3-year-olds, all SAT dimensions (except for avoidance) correlated positively with a greater tendency to trust in the human, including during the trust loss phase. For 7-year-olds, quality of attachment positively correlated with the tendency to trust during the trust loss phase.

For 9-year-olds, the results also showed a positive relationship between trust acquisition and the SAT sub-dimension of attachment, indicating that more securely attached children tended to acquire trust quicker. Additionally, among 9year-olds, there was a positive correlation between the tendency to trust during the trust loss phase and the SAT sub-dimension of avoidance. This correlation was also significant across ages.

A positive correlation was finally found between the SAT sub-dimension of attachment and the tendency to trust in the robot for 3-year-olds during the restoration of trust phase. No significant correlations were found for 5 -year-olds.

\section{Trusting and ToM}

The scores on the two ToM tasks were merged into one single score for each level of complexity (first and second order).
A low level of ToM performance (coded 0) included children who scored 0 (failed) on both tasks, whereas a high level of performance (coded 1) included children who passed at least one ToM task at each complexity level. Table 2 reports descriptive data for the ToM tasks.

All correlations found between the tendency to trust and ToM scores were negative. Thus, greater ToM abilities were associated with a lower tendency to trust, i.e. with a more reflective tendency to trust. This relationship was independent of the partner's agency (human or robot) or the child's age. The tendency to trust was often significantly correlated with first-order ToM, which was therefore included as a covariate in the GLM model described above. Finally, a substantial negative correlation between the tendency to trust and second-order ToM was observed during the acquisition of trust for children aged 7 years when playing with the human. These statistics are reported in Table 3.

\section{Trusting and Executive Function Skills}

Children aged 3 and 5 years were administered the DCCS, which assesses the capacity to switch between responses (Zelazo, 2006). The same skill was assessed in 7- and 9year-olds using the "Developmental NEuroPSYchological Assessment" subtest (NEPSY II; Korkman et al., 2007). To compare data across age groups, scores were standardized.

Significant age-related positive relationships were found between the ability to switch and the tendency to trust during the restoration phase among 3-year-olds when playing with the human, $r(19)=0.49, P<0.05$, and among 7 -year-olds when playing with the robot, $r(22)=0.43, P<0.05$.

TABLE 1| Association between Trust and SAT.

SAT sub-dimensions

\begin{tabular}{|c|c|c|c|c|c|c|c|c|c|}
\hline \multirow[b]{2}{*}{ Trust phase } & \multirow[b]{2}{*}{ Age group $(N)$} & \multicolumn{4}{|c|}{ Playing with human } & \multicolumn{4}{|c|}{ Playing with robot } \\
\hline & & Attachment & Self-confidence & Avoidance & тот & Attachment & Self-confidence & Avoidance & TOT \\
\hline \multirow[t]{3}{*}{ Acquisition } & 5 years (20) & -0.042 & -0.227 & 0.088 & -0.205 & 0.282 & 0.007 & -0.004 & 0.1 \\
\hline & 7 years (22) & 0.2 & 0.008 & -0.264 & 0.302 & -0.135 & 0.063 & -0.12 & -0.06 \\
\hline & 9 years (23) & $0.477^{\star}$ & -0.118 & -0.306 & 0.259 & 0.412 & -0.003 & 0.056 & 0.224 \\
\hline \multirow[t]{4}{*}{ Loss } & 5 years (20) & -0.195 & -0.012 & 0.023 & -0.095 & 0.198 & -0.025 & 0.138 & -0.014 \\
\hline & 7 years (22) & $0.526^{\star}$ & 0.066 & 0.068 & 0.412 & 0.188 & 0.153 & 0.247 & -0.021 \\
\hline & 9 years (23) & -0.177 & -0.273 & $0.487^{\star}$ & -0.262 & 0.048 & 0.209 & -0.106 & 0.187 \\
\hline & Overall & -0.146 & 0.092 & $0.218^{\star}$ & -0.14 & 0.07 & 0.158 & 0.052 & 0.007 \\
\hline (C) & 3 years (17) & 0.298 & 0.418 & 0.039 & 0.163 & $0.629^{\star \star}$ & 0.459 & -0.307 & 0.48 \\
\hline Restoration & Overall & -0.066 & 0.128 & 0.05 & 0.005 & 0.203 & 0.205 & -0.08 & 0.209 \\
\hline
\end{tabular}

Correlations between the tendency to trust during (A) the acquisition, (B) loss, and (C) restoration of trust and the quality of attachment relationships (SAT), sub-dimensions (attachment, self-confidence, avoidance), as well as total SAT. ${ }^{* *}$ Correlation is significant at the level 0.01 (two-tailed). ${ }^{*}$ Correlation is significant at the level 0.05 (two-tailed). Significant values are in bold. 
TABLE 2 | TOM descriptives.

\begin{tabular}{|c|c|c|c|c|}
\hline \multirow[t]{2}{*}{ Age group (years/N) } & \multicolumn{2}{|c|}{ First-order ToM } & \multicolumn{2}{|c|}{ Second-order ToM } \\
\hline & Low (\%) & High (\%) & Low (\%) & High (\%) \\
\hline $3(22)$ & 68 & 32 & - & - \\
\hline $5(24)$ & 25 & 75 & 50 & 50 \\
\hline $7(24)^{\star}$ & 0 & 96 & 20 & 76 \\
\hline $9(23)$ & 0 & 100 & 13 & 87 \\
\hline
\end{tabular}

Percentage of children in each age group (years) displaying a low or high first and second-order Theory of Mind (ToM). *One missing case.

TABLE 3 | Association between Trust and ToM.

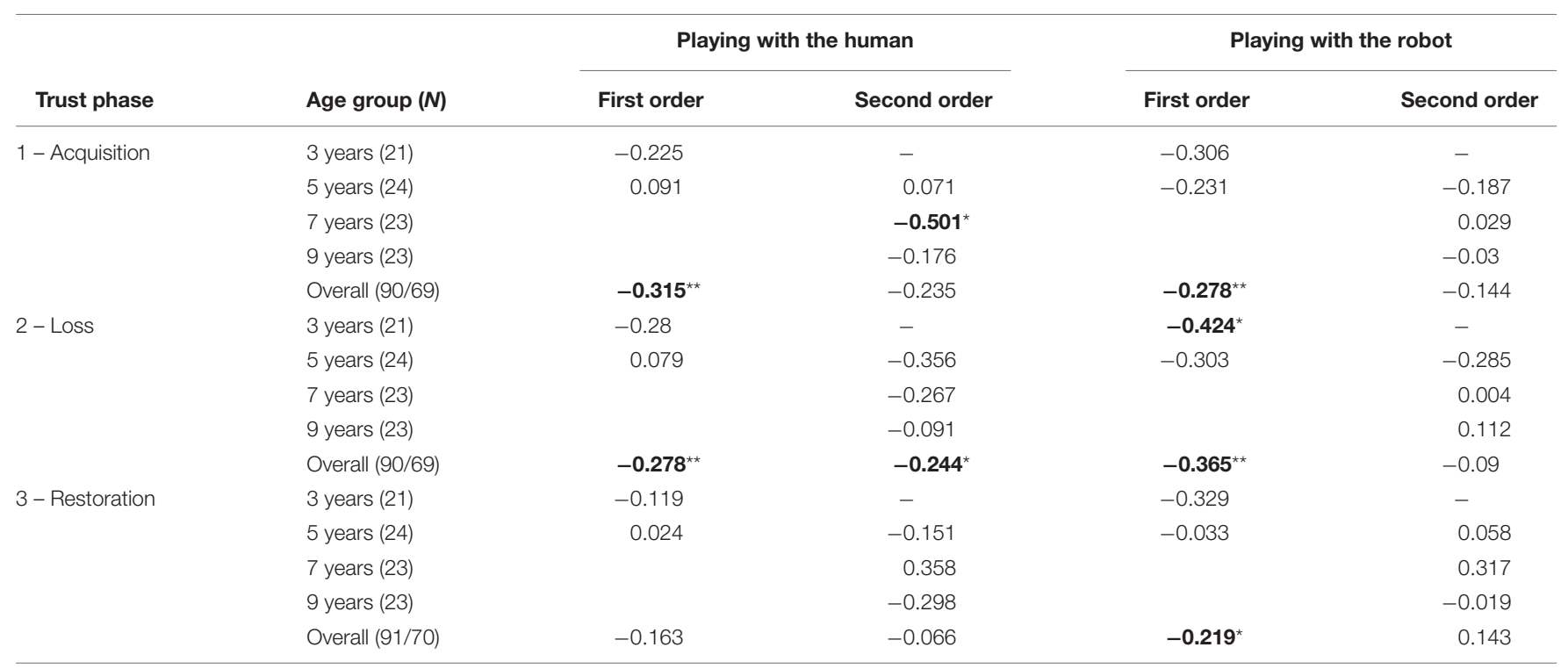

Correlations between the tendency to trust and ToM score. ${ }^{* *}$ Correlation is significant at the level 0.01 (two-tailed). ${ }^{*}$ Correlation is significant at the level 0.05 (two-tailed). First-order ToM had a constant value for age groups 7 and 9 years and correlations could not be calculated. Significant values are in bold.

\section{Attribution of Mental States}

A repeated measures GLM analysis comparing AMS scores between human and robot, with five levels of state (perceptual, emotions, intentions and desires, imagination, and epistemic) and two levels of agency (HB and RB) as within-subjects factors, and age group (four levels) as the between-subjects factor, showed a main effect of state, $F(4,332)=71.72, P<0.001$, partial$\eta^{2}=0.46, \delta=1$, a main effect of agency $(\mathrm{HB}>\mathrm{RB}), F(1$, $83)=82.10, P<0.001$, partial $-\eta^{2}=0.50, \delta=1$, an interaction of state $\times$ age group, $F(12,332)=5.18, P<0.001$, partial$\eta^{2}=0.16, \delta=1$, an interaction of agency $\times$ age group, $F(3$, $83)=8.66, P<0.001$, partial $-\eta^{2}=0.24, \delta=0.99$, an interaction of state $\times$ agency, $F(12,332)=19.99, P<0.001$, partial $-\eta^{2}=0.19$, $\delta=1$, and a three-way interaction between state, agency, and age group, $F(12,332)=2.31, P<0.01$, partial $-\eta^{2}=0.85, \delta=0.96$. This interaction is represented in Figure 3.

Exploring the three-way interaction, the most consistent difference was for the attribution of perception $(\mathrm{HB}>\mathrm{RB})$, which was significant for all four age groups, $P<0.01$. Attribution of epistemic states was also greater for $\mathrm{HB}$ than $\mathrm{RB}$ for all age groups, $P<0.01$, except 5 -year-olds, for whom there was a trend toward significance, $P=0.07$. Attributions of emotion and imagination were similar for $\mathrm{HB}$ and $\mathrm{RB}$ among 3- and 5-year-olds, but greater for $\mathrm{HB}$ among 7 - and 9-year-olds, $P<0.05$. Finally, only 9 -year-olds ascribed greater intentions and desires to HB than RB, Mdiff $=4.00 ; \mathrm{SE}, 0.63 ; P<0.001$. These post hoc analyses are summarized in Figure 3. Overall, these analyses confirm that humans and robots are differentiated even by 3-year-olds with respect to perception and epistemic states with that differentiation spreading to all five states among 9-year-olds.

\section{DISCUSSION}

The present study investigated trust dynamics when children aged $3,5,7$, and 9 years played a TG in vivo with either a human or a robot partner. Children's tendency to trust decreased across the three phases of the game, from acquisition to restoration of trust. Also, 3-year-olds displayed a greater tendency to trust in both play partners compared to the other age groups, although initially placing their trust more easily in the human than in the robot. The opposite was observed for the 7-year-olds, who generally placed more trust in the robot than the human. 


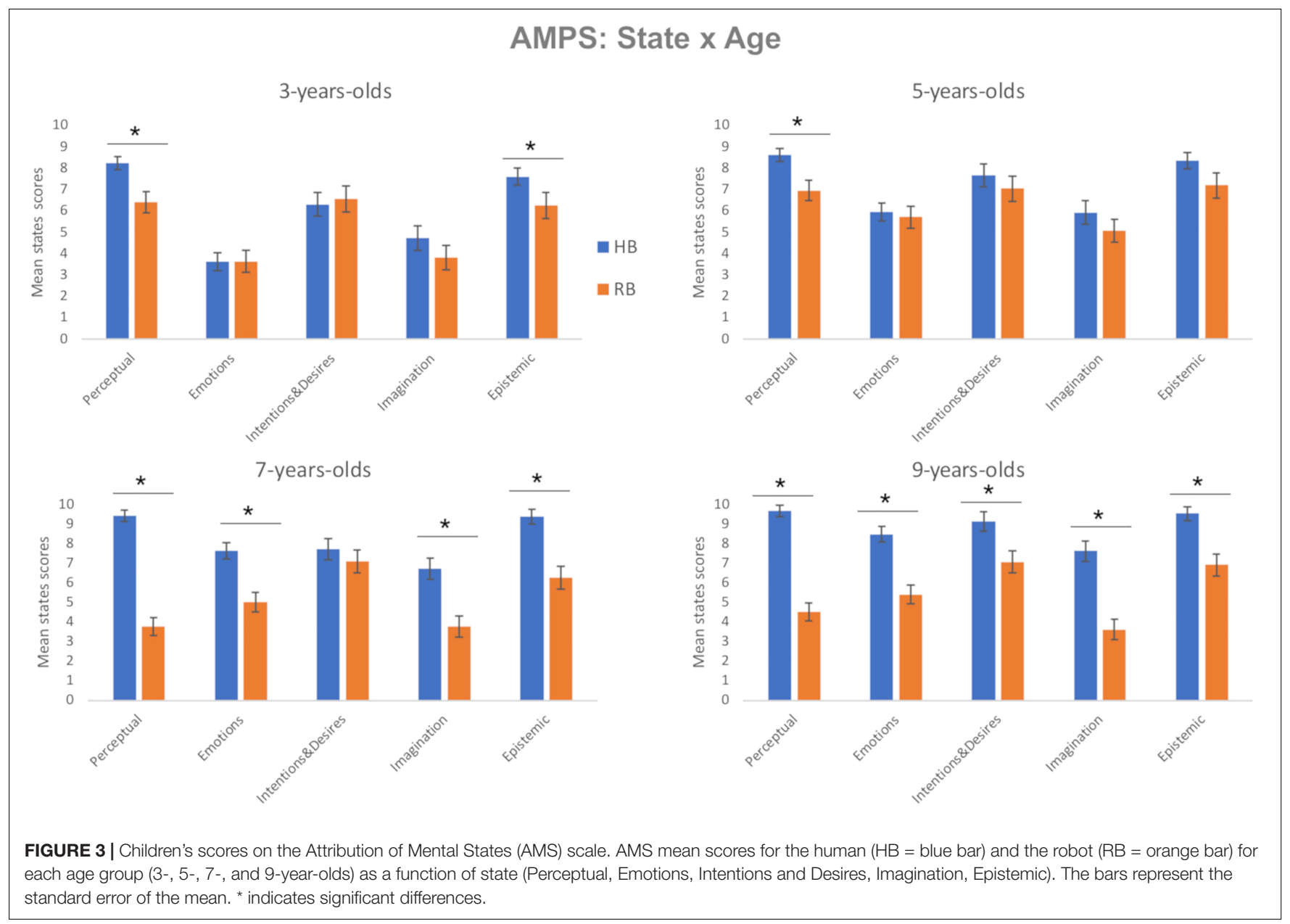

To better understand age changes in trust, the results for quality of attachment relationships, false belief understanding, and executive function skills were examined. It has been previously shown that children aged 3 and 4 years are likely to endorse information provided by someone who proved accurate in the past (see also Koenig and Harris, 2005; Pasquini et al., 2007; Harris, 2007). The results for SAT deepen this observation. Among 3-year-olds, we found that the SAT sub-dimension of self-confidence was positively associated with selective trust in the human, probably because these children's past relationships were secure, thus increasing the perception of the unfamiliar experimenter as trustworthy. On the contrary, the robot was an entity with which children had never had any relational experience, further skewing the youngest children's trust preference toward the human. Additionally, it was found that the youngest children - and particularly securely attached children - showed a tendency to retain trust during the loss of trust phase, confirming a certain behavioral rigidity as introduced above. However, when realizing that the other was no longer trustworthy, they switched to trusting the robot more. This result supports the observation that when very young children's expectations are betrayed (loss of trust), they are less forgiving than older children (Harris, 2007); additionally, our findings enrich previous results (Corriveau and Harris, 2009) by further showing that children who are securely attached in infancy are more flexible when investing their trust.

The development of a fundamental cognitive ability makes a substantial contribution to trust dynamics in child-robot interaction across all age groups. According to our findings, the development of ToM appears to temper the relation between quality of attachment relationships and trust, by introducing into the trust matrix a mentalistic evaluation of the other's judgment based on an awareness of her/his/its beliefs. More specifically, children who had developed at least first-order ToM also knew that the other player did not know the position of the doll, and was therefore an unreliable informant. Not by chance, the effect of ToM on trusting behavior was most evident at 3 and 7 years of age, typically marked by the development of increasingly complex levels of ToM. When children start developing the concept of the other's mind, they are also able to evaluate whether the other (either a human or a robot) is trustworthy on the basis of informational access. Preferential trust in either agent then moderates. 
The dichotomy found between the younger and older age groups in the AMS to the robot and the human further helps to delineate the children's perception of the robot as a mentalistic agent: For younger children, the robot is perceived as more mentalistically comparable to the human than for older children. Nevertheless, when younger children decided to trust a play partner, the affective component prevailed over the more "cool" mentalistic component, defining the preferred relational target accordingly (i.e. the human). On the other hand, the trust attributed to the robot by older children may stem from the dominance of a cognitive over an affective engagement.

In support of agent-specific differences in trusting behavior between 3- and 7-year-olds, the results also revealed a positive association between the ability to switch and trusting behavior during the trust restoration phase among children aged 3 and 7 years. Strikingly, and consistent with the data discussed above, these relations were specific to playing with the human partner for the 3-year-olds, and to playing with the robot for the 7year-olds. In general, these correlations indicate that a greater tendency to recover trust in the other is associated with the development of the ability to switch. The specificity related to the play partner's agency further underlines the relevance of the interactive partner for the child and reflects children's engagement with one or the other player: 3-year-olds' selective trust in the human was plausibly influenced by the quality of attachment relationships - as also evidenced by data on attachment described above; 7-year-olds preferential trust in the robot was possibly due to an emerging familiarity with artificial devices typical of this age. These results shed light on previous findings (Lucas et al., 2013) that showed that, compared to false belief understanding, executive functions skills do not play an essential role in building selective trust in an informant, at least within specific cultural frames. In our study, we did find that executive function skills played a role, though not during the phase of trust acquisition, but rather during trust restoration. Here, the child's ability to switch was possibly required to re-organize information and re-establish trust in an informant that, during the loss of trus phase, became unreliable. Executive function skills may then be specifically involved in building trust only under specific conditions, which had not been empirically assessed so far.

\section{CONCLUSION, LIMITATIONS, AND FUTURE DIRECTIONS}

The present study provided some insight into the dynamics of trust both when relating to a human and a robot partner. Our results highlighted the impact of cognitive development, as well as children's attachment history. We found that cognition and attachment operated separately (given the absence of a direct correlation between these two dimensions) on the establishment of trust. Particularly for children aged 3 years, trust appears to be significantly influenced by the affective dimension of trust, especially when interacting with a human. Interestingly, although securely attached children exhibited a greater tendency to trust the human, they also shifted their trust more rapidly in the trust restoration phase with the robot. This may be due to the lack of any affective bond with the robot and to the child's cool relational attitude toward it. Effectively, this would render the robot a more "forgivable" partner.

Also, the development of false belief understanding proved to play a significant role in the establishment of trusting relationships. In particular, the development of mentalizing abilities enabled children to reflect rationally on the fact that the other player had exactly the same guessing opportunities as they did, and was therefore as susceptible to making mistakes as they were. This moderated the effect of the affective component of trust.

In the present study, the robot proved to be less susceptible to the dynamics associated with the quality of attachment relationships, and thus became a more stable trusted partner. For this reason, and particularly for children with fragile affective relational histories who have difficulties with trust, the robot might fulfill a significant scaffolding role in human-human interaction. However, an evolution of the robot as a social partner is also to be expected. Therefore, different relational dynamics may be anticipated, according to which, perhaps, an affective relation history will be created with this new entity. In this respect, a longitudinal study would further delineate the development of trust in the robot increasing the robustness of the findings. Also, a larger sample size would eventually confirm the observed tendencies.

Last, but not least, the findings from this study may inform disciplines such as Developmental Robotics on how cognitive architectures can be modeled in the robot so as to make it trusting in the human partner in a "human-like" fashion, as discussed above. This circular behavior would make the human-robot relationship increasingly ecological and, ultimately, trustful. Starting, for example, from the architectural model designed by Vinanzi et al. (2019), in which the robots' trust in an informant varied as a function ToM, the present findings clearly indicate further psychological factors that may be integrated in the robot to design the robot's trust in the human at different developmental levels. Recent technical and theoretical achievements in the field of social robotics have encouraged researchers to develop social robots as tutors and learning companions for children (e.g. Movellan et al., 2009; Tanaka and Matsuzoe, 2012; Breazeal et al., 2016). Therefore, studying the mechanisms by which children learn from robots, and vice versa, is of vital importance.

\section{DATA AVAILABILITY STATEMENT}

All data needed to evaluate the conclusions in the article are present in the article.

\section{ETHICS STATEMENT}

The studies involving human participants were reviewed and approved by the Ethic Committee, Università Cattolica del Sacro Cuore, Milano, Italy. Written informed consent to 
participate in this study was provided by the participants' legal guardian/next of kin.

\section{AUTHOR CONTRIBUTIONS}

AM, AC, PH, DM, CD, and FM conceived and designed the experiment. FM and GP conducted the experiments in schools. $\mathrm{AM}$ and FM secured ethical approval. CD and DM carried out the statistical analyses. AC granted the use of the SoftBank Robotics NAO humanoid robot. All authors contributed to the writing of the manuscript.

\section{FUNDING}

This work has been in part supported by the Honda grant "DecIFER" and the US EOARD grant "THRIVE++"

\section{REFERENCES}

Allison, E., and Fonagy, P. (2016). When is truth relevant? Psychoanal. Q. 85, 275-303. doi: 10.1002/psaq.12074

Astington, J. W., Pelletier, J., and Homer, B. (2002). Theory of mind and epistemological development: the relation between children's second-order false-belief understanding and their ability to reason about evidence. New Ideas Psychol. 20, 131-144. doi: 10.1016/s0732-118x(02)00005-3

Baxter, P., Ashurst, E., Read, R., Kennedy, J., and Belpaeme, T. (2017). Robot education peers in a situated primary school study: personalisation promotes child learning. PLoS One 12:e0178126. doi: 10.1371/journal.pone.017 8126

Belpaeme, T., Kennedy, J., Ramachandran, A., Scassellati, B., and Tanaka, F. (2018). Social robots for education: a review. Sci. Robot. 3:eaat5954. doi: 10.1126/ scirobotics.aat5954

Bo, S., Sharp, C., Fonagy, P., and Kongerslev, M. (2017). Hypermentalizing, attachment, and epistemic trust in adolescent BPD: clinical illustrations. Personal. Disord. 8, 172-182. doi: 10.1037/per0000161

Bowlby, J. (1969). Attachment. Attachment and Loss (1). New York, NY: Basic Books.

Bowlby, J. (1973). Separation: Anxiety \& Anger. Attachment and Loss (2). London: Hogarth Press.

Bowlby, J. (1980). Loss: Sadness \& Depression. Attachment and Loss (3). London: Hogarth Press.

Breazeal, C., Dautenhahn, K., and Kanda, T. (2016). Social Robotics. In Springer Handbook of Robotics (1935-1972). Cham: Springer.

Brosseau-Liard, P., Penney, D., and Poulin-Dubois, D. (2015). Theory of mind selectively predicts preschoolers' knowledge-based selective word learning. $\mathrm{Br}$. J. Dev. Psychol. 33, 464-475. doi: 10.1111/bjdp.12107

Camisasca, E., Procaccia, R., Miragoli, S., Valtolina, G. G., and Di Blasio, P. (2017). Maternal mind-mindedness as a linking mechanism between childbirth-related posttraumatic stress symptoms and parenting stress. Health Care Women Int. 38, 593-612. doi: 10.1080/07399332.2017.1296840

Cangelosi, A., and Schlesinger, M. (2015). Developmental Robotics: From Babies to Robots. Cambridge, MA: MIT Press.

Cangelosi, A., and Schlesinger, M. (2018). From babies to robots: the contribution of developmental robotics to developmental psychology. Child Dev. Perspect. 12, 183-188. doi: 10.1111/cdep.12282

Carlson, S. M., and Moses, L. J. (2001). Individual differences in inhibitory control and children's theory of mind. Child Dev. 72, 1032-1053. doi: 10.1111/14678624.00333

Chan, C. C. Y., and Tardif, T. (2013). Knowing better: the role of prior knowledge and culture in trust in testimony. Dev. Psychol. 49, 591-601. doi: 10.1037/ a0031336 to AC, and by the Universitá Cattolica del Sacro Cuore, Milan.

\section{ACKNOWLEDGMENTS}

We wish to thank Dr. Gabriella Fumagalli, Principal of the "I.C. Leonardo da Vinci", and Mrs. Fulvia Maria Frigerio for their deep involvement in the organization of and support to the study. We wish to thank Dr. Mario Uboldi, Principal of the "I.C. Giovanni Pascoli", and Mrs. Maura Panzone for their important contribution to the organization of the project and support to the study. We also wish to thank all the teachers, who actively helped in conducting the study and, in particular, the schools "Scuola dell'Infanzia Premenugo," "Scuola dell'Infanzia Rodano," "Scuola dell'Infanzia Caleppio," "Scuola Primaria Rasori," and "Scuola Primaria Settala."

Clément, F., Koenig, M., and Harris, P. (2004). The ontogenesis of trust. Mind Lang. 19, 360-379. doi: 10.1111/j.0268-1064.2004.00263.x

Corriveau, K., and Harris, P. L. (2009). Choosing your informant: weighing familiarity and recent accuracy. Dev. Sci. 12, 426-437. doi: 10.1111/j.1467-7687. 2008.00792.x

Corriveau, K. H., Harris, P. L., Meins, E., Fernyhough, C., Arnott, B., Elliott, L., et al. (2009). Young children's trust in their mother's claims: longitudinal links with attachment security in infancy. Child Dev. 80, 750-761. doi: 10.1111/j. 1467-8624.2009.01295.x

Di Dio, C., Isernia, S., Ceolaro, C., Marchetti, A., and Massaro, D. (2018). Growing up thinking of God's beliefs: theory of mind and ontological knowledge. Sage Open 8, 1-14.

Di Dio, C., Manzi, F., Itakura, S., Kanda, T., Ishiguro, H., Massaro, D., et al. (2019). It does not matter who you are: fairness in pre-schoolers interacting with human and robotic partners. Int. J. Soc. Robot. 1, 1-15.

Di Dio, C., Manzi, F., Peretti, G., Cangelosi, A., Harris, P. L., Massaro, D., et al. (2020). Come i bambini pensano alla mente del robot: il ruolo dell'attaccamento e della Teoria della Mente nell'attribuzione di stati mentali ad un agente robotico. Sist. Intell. 1, 41-56.

Fonagy, P. (1998). Attachment theory approach to treatment of the difficult patient. Bull. Menninger Clin. 62, 147-169.

Fonagy, P., Luyten, P., Allison, E., and Campbell, C. (2019). Mentalizing, epistemic trust and the phenomenology of psychotherapy. Psychopathology 52, 94-103. doi: $10.1159 / 000501526$

Fonagy, P., Redfern, S., and Charman, T. (1997). The relationship between beliefdesire reasoning and a projective measure of attachment security (SAT). Br. J. Dev. Psychol. 15, 51-61. doi: 10.1111/j.2044-835x.1997.tb00724.x

Frye, D., Zelazo, P. D., and Palfai, T. (1995). Theory of mind and rule based reasoning. Cogn. Dev. 10, 483-527. doi: 10.1016/0885-2014(95)90 024-1

Fusaro, M., and Harris, P. L. (2008). Children assess informant reliability using bystanders' non-verbal cues. Dev. Sci. 11, 771-777. doi: 10.1111/j.1467-7687. 2008.00728.x

Gaudiello, I., Zibetti, E., Lefort, S., Chetouani, M., and Ivaldi, S. (2016). Trust as indicator of robot functional and social acceptance. An experimental study on user conformation to iCub answers. Comput. Human Behav. 61, 633-655. doi: 10.1016/j.chb.2016.03.057

Giovanelli, C., Di Dio, C., Lombardi, E., Tagini, A., Meins, E., Marchetti, A., et al. (2020). Exploring the relation between maternal mind-mindedness and children's symbolic play: a longitudinal study from 6 to 18 months. Infancy 25 , 67-83. doi: 10.1111/infa.12317

Grover, S. L., Abid-Dupont, M.-A., Manville, C., and Hasel, M. C. (2019). Repairing broken trust between leaders and followers: how violation characteristics temper apologies. J. Bus. Ethics 155, 853-870. doi: 10.1007/s10551-017-3509-3 
Hafford-Letchfield, T., and Spatcher, P. (2007). Getting to know you: social work students experiences of direct work with children in education settings. Soc. Work Educ. 26, 311-317. doi: 10.1080/02615470601049891

Hancock, P. A., Billings, D. R., Schaefer, K. E., Chen, J. Y., de Visser, E. J., and Parasuraman, R. (2011). A meta-analysis of factors affecting trust in humanrobot interaction. Hum. Factors 53, 517-527. doi: 10.1177/0018720811417254

Hansburg, H. G. (1972). Separation Problems of Displaced Children. Oxford: Stanwix House.

Harris, P. L. (2007). Trust. Dev. Sci. 10, 135-138.

Heerink, M. (2010). Assessing Acceptance of Assistive Social Robots by Aging Adults. Ph.D Dissertation, Amsterdam: University of Amsterdam.

Jones, G. R., and George, J. M. (1998). The experience and evolution of trust: implications for cooperation and teamwork. Acad. Manage. Rev. 23, 531-546. doi: $10.5465 / \mathrm{amr} .1998 .926625$

Kanda, T., Hirano, T., Eaton, D., and Ishiguro, H. (2004). Interactive robots as social partners and peer tutors for children: a field trial. Hum. Comput. Interaction 19, 61-84. doi: 10.1207/s15327051hci1901\%262_4

Kidd, C. D. (2003). Sociable Robots: The Role of Presence and Task in Human-Robot Interaction. Unpublished Master Thesis, Boston, MA: Massachusetts Institute of Technology.

Klagsbrun, M., and Bowlby, J. (1976). Responses to separation from parents: a clinical test for young children. Br. J. Proj. Psychol. Pers. Study 21, 7-27.

Koenig, M. A., Clément, F., and Harris, P. L. (2004). Trust in testimony: children's use of true and false statements. Psychol. Sci. 15, 694-698. doi: 10.1111/j.09567976.2004.00742.x

Koenig, M. A., and Harris, P. L. (2005). Preschoolers mistrust ignorant and inaccurate speakers. Child Dev. 76, 1261-1277. doi: 10.1111/j.1467-8624.2005. 00849.x

Korkman, M., Kirk, U., and Kemp, S. (2007). NEPSY-II: A Developmental Neuropsychological Assessment. San Antonio, TX: The Psychological Corporation.

Kuchenbrandt, D., and Eyssel, F. (2012). "The mental simulation of a human robot interaction: positive effects on attitudes and anxiety toward robots," in Proceedings of the 21st IEEE International Symposium in Robot and Human Interactive Communication (RO-MAN 2012), Paris.

Lahno, B. (2001). On the emotional character of trust. Cultivat. Emot. 4, 171-189.

Lecciso, F., Petrocchi, S., Liverta Sempio, O., and Marchetti, A. (2011). A contribution to a new tool of the relationships between affects and mentalization: the trust story. Psicol. Clin. Dello Sviluppo 15, 63-94.

Lewis, M., Sycara, K., and Walker, P. (2018). "The role of trust in human-robot interaction" In Foundations of Trusted Autonomy eds H. A., Abbass, J., Scholz, D. J., Reid. Cham: Springer, 135-159.

Liverta Sempio, O., Marchetti, A., and Lecciso, F. (2001). Il SAT Famiglia e il SAT Scuola: Strumenti di Misura dell'Ansia da Separazione da Genitori ed Insegnanti. Milan: Università Cattolica del Sacro Cuore, ISU.

Lombardi, E., Di Dio, C., Castelli, I., Massaro, D., and Marchetti, A. (2017). Prospective thinking and decision making in primary school age children. Heliyon 3:e00323. doi: 10.1016/j.heliyon.2017.e00323

Lombardi, E., Greco, S., Massaro, D., Schär, R., Manzi, F., Iannaccone, A., et al. (2018). Does a good argument make a good answer? Argumentative reconstruction of children's justifications in a second order false belief task. Learn. Cult. Soc. Interact. 18, 13-27. doi: 10.1016/j.lcsi.2018.02.001

Lucas, A. J., Lewis, C., Pala, F. C., Wong, K., and Berridge, D. (2013). Socialcognitive processes in preschoolers' selective trust: three cultures compared. Dev. Psychol. 49, 579-590. doi: 10.1037/a0029864

Luo, Y., Hétu, S., Lohrenz, T., Hula, A., Dayan, P., Ramey, S. L., et al., (2018). Early childhood investment impacts social decision-making four decades later. Nat. Commun. 9:4705. doi: 10.1038/s41467-018-07138-5

Manzi, F., Di Dio, C., Itakura, S., Kanda, T., Ishiguro, H., Massaro, D., et al. (2020). "Moral evaluation of human and robot interactions in Japanese preschoolers," in ACM IUI Workshop Proceedings, Cagliari.

Marchetti, A., Manzi, F., Itakura, S., and Massaro, D. (2018). Theory of mind and humanoid robots from a lifespan perspective. Zeitschrift für Psychologie 226, 98-109. doi: 10.1027/2151-2604/a000326

Marchetti, A., Miraglia, L., and Di Dio, C. (2020). Towards a socio-material approach to cognitive empathy in Autistic Spectrum Disorder. Front. Psychol. 10:2965. doi: 10.3389/fpsyg.2019.02965

Martini, M. C., Gonzalez, C. A., and Wiese, E. (2016). Seeing minds in othersCan agents with robotic appearance have human-like preferences. PLoS One 11:e0146310. doi: 10.1371/journal.pone.0146310
Movellan, J., Eckhardt, M., Virnes, M., and Rodriguez, A. (2009). "Sociable robot improves toddler vocabulary skills," in Proceedings of the 2009 4th ACM/IEEE International Conference on Human-Robot Interaction (HRI), (La Jolla, CA: IEEE).

Nass, C., and Moon, Y. (2000). Machines and mindlessness: social responses to computers. J. Soc. Issues 56, 81-103. doi: 10.1111/0022-4537.00153

Nass, C., Moon, Y., Fogg, B. J., and Reeves, B. (1995). Can computer personalities be human personalities? Int. J. Human Comput. Stud. 43, 223-239. doi: 10. 1006/ijhc.1995.1042

Okumura, Y., Kanakogi, Y., Kanda, T., Ishiguro, H., and Itakura, S. (2013a). Infants understand the referential nature of human gaze but not robot gaze. J. Exp. Child Psychol. 116, 86-95. doi: 10.1016/j.jecp.2013.02.007

Okumura, Y., Kanakogi, Y., Kanda, T., Ishiguro, H., and Itakura, S. (2013b). The power of human gaze on infant learning. Cognition 128, 127-133. doi: 10.1016/ j.cognition.2013.03.011

Pasquini, E. S., Corriveau, K. H., Koenig, M., and Harris, P. L. (2007). Preschoolers monitor the relative accuracy of informants. Dev. Psychol. 43, 1216-1226. doi: 10.1037/0012-1649.43.5.1216

Perner, J., and Wimmer, H. (1985). "John thinks that mary thinks that..." attribution of second-order beliefs by 5-to 10-year-old children. J. Exp. Child Psychol. 39, 437-471. doi: 10.1016/0022-0965(85)90051-7

Petrocchi, S., Rotenberg, K. J., Levante, A., and Lecciso, F. (2018). Children's trust in social workers: scale development and relations to Children's engagement with social workers. Child Fam. Soc. Work 23, 239-247. doi: 10.1111/cfs. 12410

Premack, D., and Woodruff, G. (1978). Does the chimpanzee have a theory of mind. Behav. Brain Sci. 1, 515-526. doi: 10.1017/s0140525x00076512

Rizzato, M., Di Dio, C., Fasano, F., Gilli, G., Marchetti, A., and Sensidoni, A. (2016). Is food desirability affected by social interaction? Food Qual. Prefer. 50, 109-116. doi: 10.1016/j.foodqual.2016.02.005

Rotenberg, K. J. (2010). "The conceptualization of interpersonal trust: a basis, domain, and target framework," in Interpersonal Trust During Childhood and Adolescence, ed. K. J. Rotenberg (New York, NY: Cambridge University Press), $2-27$.

Rotenberg, K. J., Petrocchi, S., Lecciso, F., and Marchetti, A. (2015). The relation between children's trust beliefs and theory of mind abilities. Infant Child Dev. 24, 206-214. doi: 10.1002/icd.1891

Sharp, C., Pane, H., Ha, C., Venta, A., Patel, A. B., Sturek, J., et al. (2011). Theory of mind and emotion regulation difficulties in adolescents with borderline traits. J. Am. Acad. Child Adolesc. Psychiatry. 50, 563-573. doi: 10.1016/j.jaac.2011.01.017

Shaw-Garlock, G. (2009). Looking forward to sociable robots. Int. J. Soc. Robot. 1, 249-260. doi: 10.1007/s12369-009-0021-7

Short, E., Hart, J., Vu, M., and Scassellati, B. (2010). "No fair!! an interaction with a cheating robot," in proceedings of the 2010 th ACM/IEEE International Conference on Human-Robot Interaction (HRI), (Osaka: IEEE).

Tanaka, F., and Matsuzoe, S. (2012). Children teach a care-receiving robot to promote their learning: field experiments in a classroom for vocabulary learning. J. Hum. Robot Interact. 1, 78-95. doi: 10.5898/jhri.1.1.tanaka

Van Reet, J. (2015). Conflict inhibitory control facilitates pretense quality in young preschoolers. J. Cogn. Dev. 16, 333-350. doi: 10.1080/15248372.2013.83 3924

Van Reet, J., Green, K. F., and Sobel, D. M. (2015). Preschoolers' theory-of-mind knowledge influences whom they trust about others' theories of mind. J. Cogn. Dev. 16, 471-491. doi: 10.1080/15248372.2014.892875

Vinanzi, S., Patacchiola, M., Chella, A., and Cangelosi, A. (2019). Would a robot trust you? Developmental robotics model of trust and theory of mind. Philos. Trans. R. Soc. B 374:20180032. doi: 10.1098/rstb.2018. 0032

Vogt, P., De Haas, M., De Jong, C., Baxter, P., and Krahmer, E. (2017). Child-robot interactions for second language tutoring to preschool children. Front. Hum. Neurosci. 11:73. doi: 10.3389/fnhum.2017.00073

Wellman, H. M., Cross, D., and Watson, J. (2001). Meta-analysis of theory-ofmind development: the truth about false belief. Child Dev. 72, 655-684. doi: 10.1111/1467-8624.00304

Wiese, E., Metta, G., and Wykowska, A. (2017). Robots as intentional agents: using neuroscientific methods to make robots appear more social. Front. Psychol. 8:1663. doi: 10.3389/fpsyg.2017.01663

Wimmer, H., and Perner, J. (1983). Beliefs about beliefs: representation and constraining function of wrong beliefs in young children's understanding 
of deception. Cognition 13, 103-128. doi: 10.1016/0010-0277(83)90 004-5

Wykowska, A., Chaminade, T., and Cheng, G. (2016). Embodied artificial agents for understanding human social cognition. Philos. Trans. R. Soc. B Biol. Sci. 371:20150375. doi: 10.1098/rstb.2015.0375

Yagoda, R. E., and Gillan, D. J. (2012). You want me to trust a ROBOT? the development of a humanerobot interaction trust scale. Int. J. Soc. Robot. 4, 235-248. doi: 10.1007/s12369-012-0144-0

Yang, Y., Tian, Y., Fang, J., Lu, H., Wei, K., and Yi, L. (2017). Trust and deception in children with autism spectrum disorders: a social learning perspective. J. Autism Dev. Disord. 47, 615-625. doi: 10.1007/s10803-0162983-2

Zanatto, D., Patacchiola, M., Cangelosi, A., and Goslin, J. (2019). Generalisation of Anthropomorphic stereotype. Int. J. Soc. Robot. 2019, $1-10$.
Zelazo, P. D. (2006). The dimensional change card sort (DCCS): a method of assessing executive function in children. Nat. Protoc. 1, 297-301. doi: 10.1038/ nprot.2006.46

Conflict of Interest: The authors declare that the research was conducted in the absence of any commercial or financial relationships that could be construed as a potential conflict of interest.

Copyright (c) 2020 Di Dio, Manzi, Peretti, Cangelosi, Harris, Massaro and Marchetti. This is an open-access article distributed under the terms of the Creative Commons Attribution License (CC BY). The use, distribution or reproduction in other forums is permitted, provided the original author(s) and the copyright owner(s) are credited and that the original publication in this journal is cited, in accordance with accepted academic practice. No use, distribution or reproduction is permitted which does not comply with these terms. 


\title{
Imitation in Chinese Preschool Children: Influence of Prior Self-Experience and Pedagogical Cues on the Imitation of Novel Acts in a Non-Western Culture
}

\author{
Zhidan Wang ${ }^{1 *}$ and Andrew N. Meltzoff ${ }^{2}$ \\ 'School of Education Science, Jiangsu Normal University, Xuzhou, China, ${ }^{2}$ Institute for Learning \& Brain Sciences, University \\ of Washington, Seattle, WA, United States
}

OPEN ACCESS

Edited by: Federico Manzi,

Catholic University of the Sacred

Heart, Italy

Reviewed by:

Nicola McGuigan,

University of the West of Scotland,

United Kingdom

Jonathan T. Delafield-Butt,

University of Strathclyde, United Kingdom

*Correspondence:

Zhidan Wang

zwang19@jsnu.edu.cn

Specialty section: This article was submitted to

Developmental Psychology,

a section of the journal

Frontiers in Psychology

Received: 21 November 2019 Accepted: 19 March 2020

Published: 15 April 2020

Citation:

Wang $Z$ and Meltzoff AN (2020)

Imitation in Chinese Preschool

Children: Influence of Prior

Self-Experience and Pedagogical Cues on the Imitation of Novel Acts

in a Non-Western Culture.

Front. Psychol. 11:662.

doi: 10.3389/fpsyg.2020.00662
Both prior experience and pedagogical cues modulate Western children's imitation. However, these factors have not been systematically explored together within a single study. This paper explored how these factors individually and together influence imitation using 4-year-old children born and reared in mainland China $(N=210)$-a country that contains almost one-fifth of the world's population, and in which childhood imitation is under-studied using experimental methodology. The behavior of children in this culture is of special interest to theory because traditional East Asian culture places high value on conformity and fitting in with the group. Thus, high-fidelity imitation is emphasized in the local culture. This value, practice, or norm may be recognized by children at a young age and influence their imitative performance. In this study, we crossed prior self-experience and pedagogical cues, yielding four demonstration groups in addition to a control group. This design allowed us to investigate the degree to which Chinese preschoolers' imitation was modulated by the two experimental factors. High-fidelity imitation was significantly modulated by prior self-experience but not by pedagogical cues, as measured by the number of novel acts imitated and also the serial order of these acts. This study (i) expands our understanding of factors that modulate imitation of novel behaviors in preschoolers and (ii) contributes to efforts to broaden research beyond Western societies to enrich our theories, particularly regarding social learning and imitation. Imitation is a key mechanism in the acquisition of culturally appropriate behaviors, mannerisms, and norms but who, what, and when children imitate is malleable. This study points to both cross-cultural invariants and variations to provide a fuller picture of the scope and functions of childhood imitation.

Keywords: Chinese culture, imitation, preschool children, social cognition, observational learning, social learning, novel behaviors, over-imitation

\section{INTRODUCTION}

Imitation plays an important role in early social-cognitive development (e.g., Legare, 2017; Meltzoff and Marshall, 2018; Tomasello, 2019). Preschool children are adept at imitating novel acts that they see others perform, including using objects in particular ways, moving their bodies, the serial order in which behaviors are performed, and employing tools (e.g., Want and Harris, 2001; 
Carpenter et al., 2002; Nielsen, 2006; Nadel, 2014; Subiaul et al., 2015; Loucks et al., 2017). This human proclivity to imitate the specific details of others' behavior may have played evolutionary roles in (i) binding early humans to their social groups and (ii) supporting the diffusion of new instrumental behaviors from one person to another (e.g., how to create and use a stone tool or build a fire). In modern humans, imitative learning continues to play a prominent role in the rapid and flexible transfer of nonlinguistic information from caretakers to children. Meltzoff et al. (2009) summarized the value of imitation for child learning: It is faster than Skinnerian shaping and conditioning by caretakers; safer than trial-and-error learning by the child; and more responsive to the social-environmental context than children's individual invention.

The capacity to imitate novel behavior is especially important if imitation is to fulfill its theorized role in development. If children were constrained to duplicating only familiar acts that they had already mastered before watching the model, imitation would not enjoy the status it has in theories of developmental science, pedagogy, and human evolution. If this constraint was in place, children could not learn new social customs, behaviors, rituals, or practices from watching others, nor learn how to use novel tools to achieve instrumental ends. The imitation of novel acts is a Rosetta stone for investigating the nature and functional value of imitation in childhood (Meltzoff, 1988b; Meltzoff and Marshall, 2018).

Imitation is a mechanism for learning new behaviors, but children do not imitate everything they see all the time. Scientists have become increasingly interested in the scope of activities that children duplicate and the factors that modulate the expression of imitation. For example, there is a distinction made between re-enacting an outcome achieved by an adult (often dubbed "emulation") versus imitating the particular means and specific acts used to achieve that end (e.g., Tomasello et al., 1993). In one early investigation, it was shown that 14-month-old children re-enacted not only outcomes, but also the distinctly unusual movements and means used by adults. In this study, Meltzoff (1988a) found that infants would imitate the novel act of turning on a light panel by tapping it with their forehead after seeing an adult perform that novel act. Thus, children imitated the specific act or means demonstrated by the adult even though it was unusual, not causally necessary, and unlikely to occur by chance (the head-touch action did not occur in either of the two control groups tested). Following this report of imitation of a completely novel act, a large range of studies, using different procedures and tasks, has explored children's proclivity to imitate novelty across different ages and situations.

One prominent line of work has shown that young children will duplicate unusual behaviors when these acts are unnecessary, irrelevant, and even counterproductive for achieving a desirable physical outcome (e.g., Lyons et al., 2007, 2011; McGuigan et al., 2007; Nielsen and Tomaselli, 2010; Hoehl et al., 2014). This tendency has been referred to as "over-imitation" (although this term itself has been questioned, inasmuch as the word "over" might be misleading; the research may be thought of as investigating the imitation of novel acts and the conditions under which children exhibit imitation of such behaviors even when they are not necessary for achieving a physical-instrumental end). In terms of theory, Lyons et al. (2007) originally proposed that "over-imitation" is a manifestation of an automatic and compulsory tendency to imitate in the human child. This process has been dubbed automatic causal encoding (ACE). The ACE claim is based on the observation that children will over-imitate despite being capable of identifying and skipping these irrelevant actions, and even though they will acknowledge that such actions are unnecessary if asked (Lyons et al., 2011). However, a different view about over-imitation is that it is an act of social affiliation between the child and the demonstrator (e.g., Nielsen and Blank, 2011; Over and Carpenter, 2012). A third view is that overimitation is driven by a motivation to adhere to apparent social norms (Kenward et al., 2011; Kenward, 2012; Keupp et al., 2013). Within this literature it has also been noted that studies examining over-imitation often use arbitrary actions with no obvious cause-effect relation with the outcome (dubbed "causally opaque"), which may influence copying.

Regardless of these theoretical debates about the meaning and motivation of "over-imitation," other researchers, working from different theoretical orientations have focused on the fact that young children's imitation of novel acts is not compulsory but rather can be highly selective (e.g., DiYanni and Kelemen, 2008; Williamson et al., 2008; Meltzoff and Williamson, 2013; Yu and Kushnir, 2014). This selectivity has captured the attention of theorists, because it highlights the agentive, active, and interpretive aspects of imitation. In one example, Clegg and Legare (2016) showed that children replicated irrelevant actions demonstrated as part of making a bead necklace (e.g., using each bead to touch forehead) only when the task was coupled with normative framing (e.g., "everyone here always does this") but not otherwise. This weighs against automaticity and favors the selectivity and modulation of the imitation of novel acts. Similarly, it has been reported that children's novel- and overimitation is dampened when the demonstrator is absent (Nielsen and Blank, 2011), is a single peer or a puppet (McGuigan and Robertson, 2015), does not belong to the same assigned group as the child (Schleihauf et al., 2019; Wilks et al., 2019), and is, herself, the target of discrimination or prejudice (Skinner et al., 2017, 2019). This line of work suggests that children are not automatically and blindly copying, but rather that there is agency and selectivity involved. From this perspective, childhood imitation is more properly thought of as malleable, modulated, and related to the interpretive context-as an active choice driven by social-cognitive factors-rather than blind, rote, and uncontrollable; in other words, "children choose whom, when, and what to imitate" (Meltzoff et al., 2009, p. 285; Meltzoff and Marshall, 2018).

Several factors have been postulated to modulate children's high-fidelity imitation of novel acts. This paper explores two such factors in a systematic way in a sample of preschool children born and raised in China-a culture that contains almost one-fifth of the world's population and with socialization practices that differ in important ways from Western culture. Comprehensive and generalizable theories of imitation cannot be advanced without knowing more about imitation among children reared 
in this culture. Claims about childhood imitation in general are incomplete if they do not test or consider imitation in traditional East Asian cultures such as China. One rationale for the current work is to broaden our understanding of factors modulating imitation of novel acts in a non-Western sample. In addition to age, at least two directly manipulable factors have been proposed to modulate children's high-fidelity imitation of novel actsone of these focuses on what the child brings to the imitation situation (aspects of their own experience and agency) and the other focuses on what the adult brings (aspects of pedagogy).

\section{Prior Self-Experience}

Results from a series of recent studies have suggested that preschool children's imitation of novel target acts is influenced by the children's own prior self-experience (Williamson et al., 2008; Williamson and Meltzoff, 2011; Wood et al., 2013; Schleihauf et al., 2018; see also Nielsen et al., 2012). For example, in Williamson et al.'s (2008) study, preschool children were randomly assigned to two prior-experience groups. In one, children had prior self-experience that the goal was easily achievable by them; in the other, children had experience that made the goal difficult to achieve (a trick mechanism made a box easy/hard to open). Following this self-experience, the children saw the adult perform an causally unrelated act (e.g., moving a toggle switch) en route to achieving the goal of opening the box. Results revealed that children who had prior self-experience of easily achieving the goal using their own means were less likely to faithfully imitate the adult's unusual action. (Children assigned to having difficulty achieving the goal were more likely to imitate the unusual action they saw, seemingly motivated to try something new). The authors theorized that children's prior selfexperience modulated children's proclivity to imitate the unusual acts. The nature of children's self-experience was postulated to set up "priors" that influenced children's imitation.

\section{Pedagogical Cues}

A second factor that has been argued to modulate children's highfidelity imitation of novel acts is pedagogical cues (e.g., adult initiated mutual eye contact, child-directed speech, Csibra and Gergely, 2009) that may indicate that the adult is trying to teach the child (e.g., Nielsen, 2006; Buchsbaum et al., 2011; Király et al., 2013). For example, Király et al. (2013) replicated Meltzoff's (1988a) head-touch study and reported that 14-month-olds copy the novel, relatively inefficient head-touch act more frequently after observing a communicative model doing this action than after incidentally observing a non-communicative model. The authors proposed that pedagogical cues, such as direct communication and ostensive signals may support children's imitation. Other researchers have downplayed the necessity of pedagogical cues. In Hoehl et al. (2014) 5-year-olds imitated causally unnecessary actions both when they were modeled by a communicative/pedagogical experimenter and when they were not. In Schmidt et al. (2011), 3-year-olds saw an adult perform a novel action without producing any ostensive cues, and yet the children imitated.

Although a number of experiments have documented the influence of prior experience and/or pedagogical cues in separate studies, to the best of our knowledge no research to date has been designed to explore children's relative weighing of prior self-experience and pedagogical cues by systematically crossing these factors within the same study in the same age group. Nor have the effects of these two factors been systematically studied in children born and raised outside of traditional Western cultures. Without this work, generalized inferences for developmental theory remain somewhat limited.

\section{Rationale and Novelty of the Study}

We investigated the role of prior self-experience and pedagogical cues on children's high-fidelity imitation of novel acts. Following the call for scientists to increase the use of participants from outside of Western, Educated, Industrialized, Rich Democratic (dubbed WEIRD) societies (Henrich et al., 2010), we tested preschool children in China-a culture that highlights and values conformity and duplication of the actions of teachers and parents. If the role of prior self-experience generalizes beyond Western cultures, we would predict more high-fidelity imitation of causally irrelevant, novel acts for children who lacked prior routines or habits for manipulating these objects. This is based on the idea that the uncertainty of what to do with the novel object makes children more attuned to adopting the specific acts and techniques demonstrated by the adult.

We also examined the degree to which pedagogical cues affect imitation of novel acts in this same study. This is of interest because it has been established that most Chinese parents tend to interact with young children in a "more authoritarian" manner than do Western parents, expecting more conformity and obedience to cultural ways of doing things (Chao, 1994; Chen et al., 2000; Chang et al., 2003; although within-culture variation certainly also exists, Zhu and Zhang, 2008; Xu et al., 2014). In general, Chinese parents do not readily provide the pedagogical cues described in Western samples (e.g., mutual gaze and parentese) to scaffold and support each step of their children's learning. In traditional Chinese culture, parents tend to teach their children in a more regimented fashion (Chinese idiom; "bu gou yan xiao," in English, "not frivolous in talking and joking") (Shek, 2002). We hypothesized that although prior self-experience may play a more culturally invariant role in modulating children's imitation (less certainty about what to do leading to higher reliance on others), pedagogical cues may have little or no influence on high-fidelity imitation in China, because children are not socialized to need, value, or expect this kind of support.

The design of the current study expands the literature in two ways. One potential contribution is that we systematically crossed prior self-experience and pedagogical cues in a study of children in China. To date, only two experimental studies of imitation have been reported from China (Wang et al., 2015; Li et al., 2019). In Wang et al.'s (2015) study, the researchers examined whether children could categorize objects by weight after observing the adult's demonstration of such sorting behavior, and the results showed that 4-year-olds, but not 3-year-olds, imitated the categorization rule (sort visually identical objects by the hidden property of weight, which might have been interpreted by children as a social norm or convention). In Li et al.'s (2019) 
study, the researchers reported that children imitated an ingroup model's approach rather than the more efficient approach demonstrated by an outgroup model. Thus, although some work on imitation in China has been reported: (i) no study to date has examined children's imitation of novel acts in an over-imitation test paradigm, and (ii) no study in China has tested the effects of pedagogical cues and prior self-experience.

Another potential contribution is that we used a broader range of measures of imitation than have typically been used in studies of preschool imitation. We measured: (i) the duplication of the overall outcome or end-state of the adult demonstration, (ii) high-fidelity imitation of novel target acts performed en route to achieving this end-state, and (iii) the duplication of the correct serial order of these novel target acts. These multiple measures help to illuminate the scope and functions that imitation may serve in human childhood. For example, if childhood imitation is a mechanism by which culturally specific rituals and customs are acquired (e.g., Rossano, 2012; Legare and Nielsen, 2015; Legare, 2017), children would need to be attentive to and capable of imitating the serial order of behaviors (Loucks et al., 2017), because rituals often demand duplicating the order in which arbitrary acts are performed (e.g., chanting before drinking the wine). In sum, the results of the current experiment promise to expand our knowledge about the factors modulating imitation of novel acts and their serial order in preschoolers beyond those in Western culture, and thereby enrich our understanding of the functions, value, and scope of imitation in Homo sapiens.

\section{MATERIALS AND METHODS}

\section{Participants}

We tested a large sample of children in China. A total of 210 children ages 4 years (110 males; $M_{\text {age }}=51.74$ months, $S D=4.78$ months) were recruited for this study. Children were recruited from a preschool in Xuzhou city, a mid-sized town in Jiangsu Province in the eastern part of mainland China. All participants were of Han ethnicity. The study was approved by the ethics committee of Jiangsu Normal University and the procedures were carried out in accord with this approval. Written parental permission for school testing of each child was obtained, and children received a small reward for their participation (e.g., stickers).

\section{Test Environment, Design, and Materials}

Children were tested individually in a separate room at their school that contained a small table for the child and experimenter (a female, native Chinese) to sit at for the test session. Each child was randomly assigned to one of five independent groups, with $n=42$ children in each group. Within each group, children were randomly assigned in terms of (i) child's gender and (ii) order of the test objects (ABCD, BCDA, CDAB, and DCBA).

Four novel objects were manufactured based on previously published work (Gonsiorowski et al., 2016). We combined several elements together to manufacture new objects, with the dual goals of (i) making the materials look somewhat unfamiliar to the children to heighten interest and (ii) assembling materials

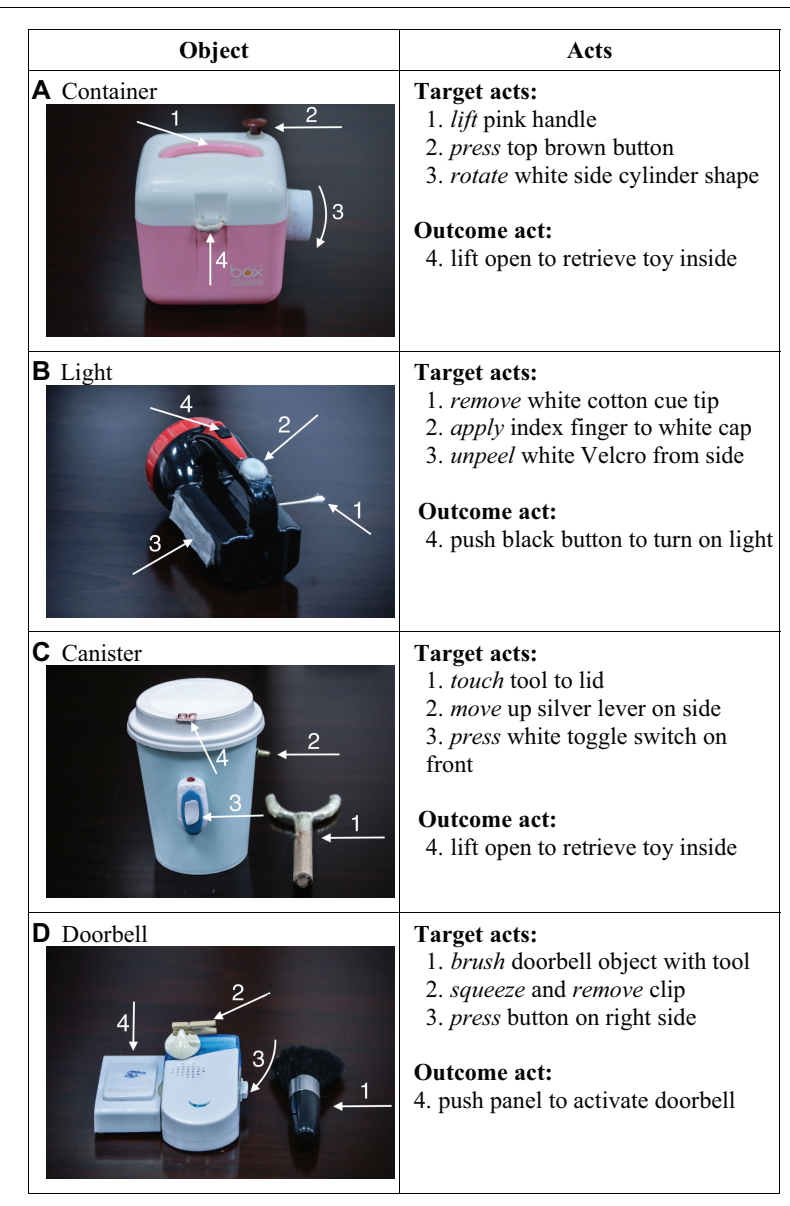

FIGURE 1 | Photographs of the four test objects (A-D), as well as verbal descriptions of the three novel target acts and the final outcome act for each object. See Figure 2 for human behaviors.

that allowed us to perform novel acts that the children would be unlikely to have seen or performed in the past (with the goal of making them relatively low-baseline acts). For example, we employed a small brush to stroke a doorbell for no apparent reason. Figure 1 displays the collection of objects and provides a description of the target acts. For each object, the experimenter performed three novel and unnecessary acts (hereafter "target acts" because these are used to measure the ability to imitate novelty) before demonstrating a final act that caused the desired outcome. A video camera was used to record the study for subsequent scoring.

\section{Procedure}

In the four treatment groups (Groups 1-4), children saw the adult demonstrations of the three novel target acts and the final outcome act. To an adult observer, the three target acts were not causally necessary to achieve the outcome. For example, the adult demonstrated the novel act of brushing a doorbell with a women's makeup-brush (designed to apply powder to the cheeks) prior to demonstrating the act of ringing the doorbell. We cannot be sure that the children construed the brushing act as non-causal 

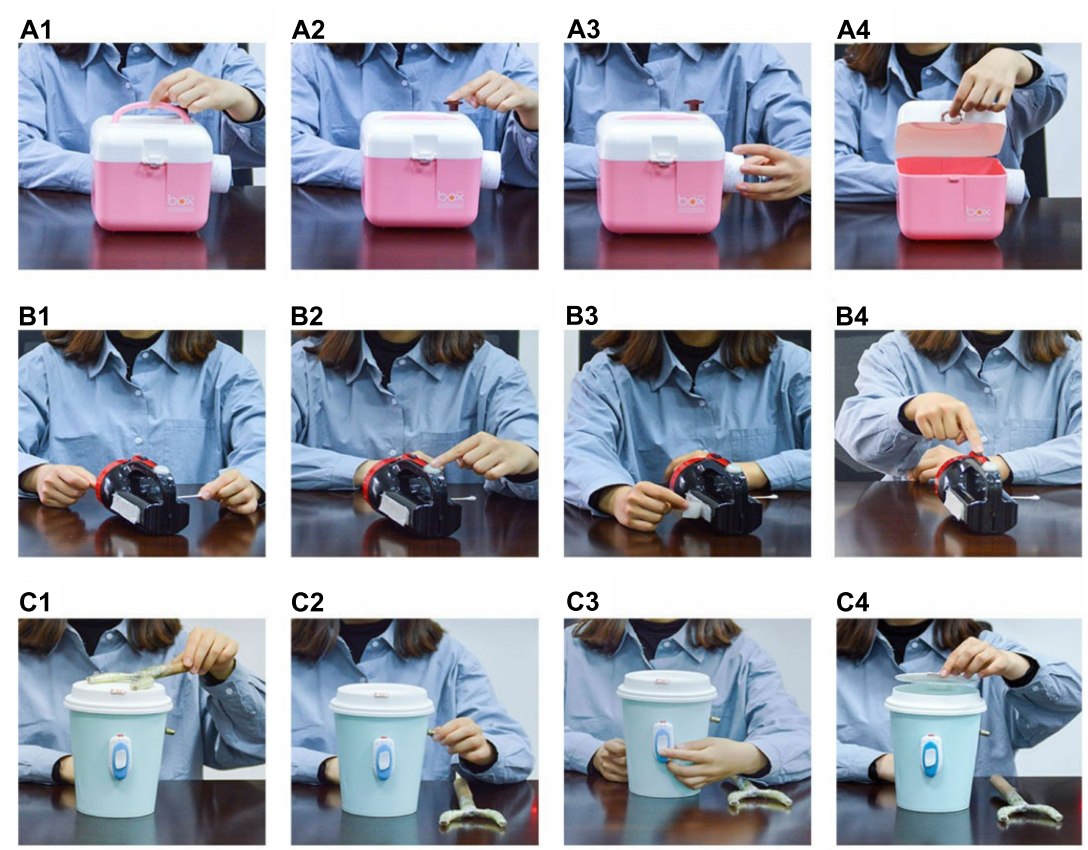

C2

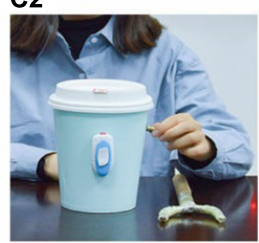

C3
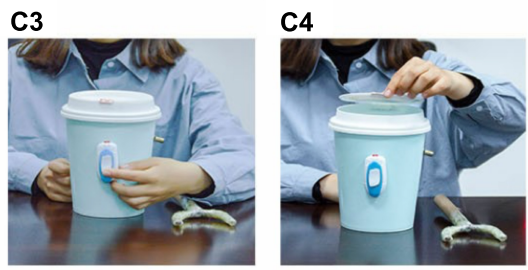

D2
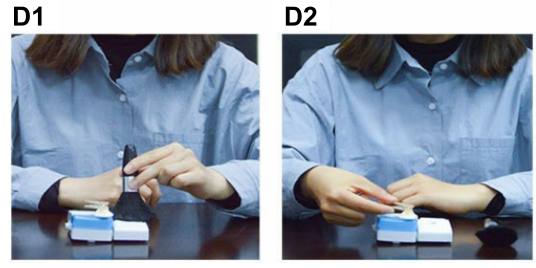

D3

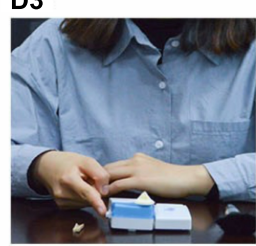

D4

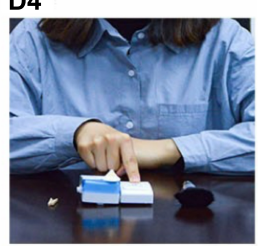

FIGURE 2 | Photographs of the four test objects (shown as the rows A1-D4). Within each row, photos show the three arbitrary target acts (Acts 1-3, in each row) and the outcome act that leads to a salient outcome (Act 4, in each row). See also Figure $\mathbf{1}$ and main text for more details.

or irrelevant to doorbell ringing, but it is justifiable to call it "novel" or arbitrary, because children have not seen someone brush a doorbell it in the past and have not been trained to perform this specific act. Each of the three novel target acts could be executed independently of one another and in any order, and were not needed to achieve the final outcome act (see Figure 2 for demonstrated behaviors). For each test object, the adult demonstrated the three target acts before performing the final outcome act.

The fifth independent group of children (Group 5) served as a baseline control in which the children received no adult demonstration before being presented with the object. This group assessed the probability that the children in the response period would spontaneous produce the target or outcome acts, in the absence of seeing them demonstrated.

\section{Demonstration Phase}

Table 1 provides an overview of the manipulations used in each of the five the independent groups used in the experiment. Procedural details are described below.

Group 1: demo(-prior + ped)

Children in this group saw the adult demonstration (indicated by the word "Demo"). They observed this demonstration along with pedagogical cues (indicated by "+ped") and without having any prior self-experience handling the test object (indicated by "-prior"). The experimenter smiled and made eye contact with the child and the tone of voice of the experimenter was warm and friendly as if "showing" or teaching the action to the child (pedagogical cues). The experimenter drew the child's attention by saying, "Today we are going to play a game. It's my turn first. Then it will be your turn." The experimenter performed the three novel target acts and then the outcome act of opening the lid to obtain the toy inside. After the demonstration, the experimenter removed the object from the children's view and reset it to the

TABLE 1 | Description of each of the five groups.

\begin{tabular}{lcccr}
\hline Group & Demonstration & $\begin{array}{c}\text { Prior } \\
\text { experience }\end{array}$ & $\begin{array}{c}\text { Pedagogical } \\
\text { cues }\end{array}$ & $\boldsymbol{n}$ \\
\hline (1) Demo(-prior +ped) & + & - & + & 42 \\
(2) Demo(-prior -ped) & + & - & - & 42 \\
(3) Demo(+prior +ped) & + & + & + & 42 \\
(4) Demo(+prior -ped) & + & + & - & 42 \\
(5) Control (baseline) & - & - & - & 42 \\
\hline
\end{tabular}

$N=210$ total. Demo, adult demonstration; ped, pedagogical cues. 
starting state and the children were handed the object for the 30-s response period (see below). Next, she re-established eye contact with the child and began a new demonstration with the next object until she had completed the demonstrations with the four test objects in one of the randomly assigned orders (e.g., ABCD).

Group-2: demo(-prior-ped)

The procedure in this group was the same as Group-1, except that the pedagogical cues were removed. Specifically, the experimenter did not smile or make eye contact with the child. When the experimenter said a sentence, the experimenter's tone of voice was neutral. During the demonstration, the experimenter's eyes remained fixed on the object rather than making eye contact with the child first.

\section{Group-3: demo $(+$ prior + ped $)$}

The procedure was the same as Group-1 except that an initial selfexperience period was added. During the self-experience phase, children were allowed to play with each object; specifically, the experimenter placed the object in front of the child and said, "Go ahead, you play with it first." The duration of the self-experience period was a fixed $30 \mathrm{~s}$, electronically timed. After this interval was complete, the experimenter asked for the object, removed it from view, and then re-presented it to the physical starting state was the same as Groups 1 and 2, and said, "Now it is my turn to play with it, Look." The remainder of the procedure was identical to Group-1.

\section{Group-4: demo(+prior-ped)}

The procedure was the same as Group-1 except that the pedagogical cues were removed, and the prior self-experience handling the object was added.

\section{Group-5: control (baseline)}

The demonstration phase was skipped for children in this group. They were administered the response period only, as described in the next section.

\section{Response Period}

The response period was the identical for all five groups. For all children, the identical protocol was followed: The adult simply handed each the object to the child to play with for an electronically timed 30-s period.

\section{Dependent Measures and Behavioral Coding}

\section{Target Act Score}

There were three novel target acts for each object (Figures 1, 2). Children obtained one point for each target act they performed on each object during the 30-s response periods. Thus, for each child, the target act scores ranged from 0 to 12 (4 objects $\times 3$ target acts).

\section{Serial Order Score}

The three novel target acts for each object were demonstrated in a serial order (Act $1 \rightarrow$ Act $2 \rightarrow$ Act 3 ). For each object, the child could copy the three target acts in the full correct order (1-2-3), or s/he could copy only two of the three target acts in the correct
TABLE 2 | Mean (SD) of dependent measures as a function of test group.

\begin{tabular}{|c|c|c|c|c|c|c|}
\hline \multirow[b]{2}{*}{ Groups } & \multicolumn{2}{|c|}{ Target acts } & \multicolumn{2}{|c|}{ Serial order } & \multicolumn{2}{|c|}{ Outcome act } \\
\hline & $M$ & (SD) & $M$ & (SD) & $M$ & $(S D)$ \\
\hline Demo(-prior +ped) & 10.26 & $(1.59)$ & 3.24 & $(1.03)$ & 3.33 & $(1.18)$ \\
\hline Demo(-prior -ped) & 9.36 & $(2.32)$ & 2.98 & $(1.07)$ & 3.50 & $(0.74)$ \\
\hline Demo(+prior +ped) & 8.95 & $(2.91)$ & 2.43 & $(1.25)$ & 3.60 & $(1.01)$ \\
\hline Demo(+prior -ped) & 8.90 & $(2.06)$ & 2.48 & $(1.23)$ & 3.67 & $(0.79)$ \\
\hline Baseline control & 4.71 & $(2.28)$ & 0.43 & $(0.83)$ & 2.62 & $(1.32)$ \\
\hline
\end{tabular}

Demo, adult demonstration; -prior, no prior self-experience; +prior, with prior selfexperience; -ped, no pedagogical cues; +ped, with pedagogical cues.

order (1-2, 2-3, or 1-3). The serial order score was a dichotomous 0 or 1 for each object. If the child copied any correctly ordered pair of acts (1-2, 2-3, or 1-3) or the entire sequence 1-2-3 for an object, s/he was scored as a 1 . If not, s/he was scored as a 0 . Thus, the total serial order score ranged from 0 to 4 (maximum score of “ 1 ” $\times 4$ objects $=4$ ).

\section{Outcome Act Score}

The child received a 1 if he or she reproduced the final outcome act for each object. Thus, the scores ranged from 0 to 4 (maximum score of “ 1 " $\times 4$ objects $=4$ ).

\section{Coding Agreement}

The primary scorer was a research assistant who remained uninformed of the participant's group assignment and the study hypotheses. A second scorer, also unaware of group assignment, coded a randomly selected $25 \%$ of the participants. Intercoder agreement was assessed by Cohen's kappa and was high for all the dependent measures (target act, $k=0.96$; serial order, $k=0.92$; outcome act, $k=0.99)$.

\section{RESULTS}

Preliminary analyses showed no significant effects of gender or object presentation order on any of the dependent measures, and thus the data were collapsed across these factors for subsequent analyses.

There was strong evidence for imitation. A one-way analysis of variance (ANOVA) was conducted using each of the three dependent measures, and the results showed that each significantly varied as a function of the experimental groups (Table 2). There was a significant effect for children's target act score, $F(4,205)=37.76, p<0.00001, \eta_{\mathrm{P}}^{2}=0.65$. Follow-up comparisons (LSD) showed that children in the Demo(-prior +ped) group $(M=10.26)$, i.e., children who had no prior experience, performed significant more target acts than did children in each of the two groups that had prior experience: Demo(+ prior + ped $), M=8.95, p=0.009$, and Demo $(+$ prior - ped), $M=8.90, p=0.007$. Moreover, there was no significant difference between the two groups with no prior experience: Demo(-prior +ped), $M=10.26$, and Demo(-prior - ped $)$, $M=9.36, p=0.069$.

There was also a significant effect as a function of group for children's serial order score, $F(4,205)=42.82, p<0.00001$, 
$\eta_{\mathrm{p}}^{2}=0.68$. Follow-up comparisons showed that children in the Demo $(-$ prior + ped) group $(M=3.24)$, i.e., children who had no prior experience, had significant higher serial order scores than did children in each of the groups that had prior experience: Demo(+prior + ped), $M=2.43, p=0.001$, and Demo $(+$ prior -ped), $M=2.48, p=0.002$. Moreover, there was no significant difference between the two groups with no prior experience: Demo(-prior +ped), $M=3.24$, and $\operatorname{Demo}(-$ prior - ped $)$, $M=2.98, p=0.274$.

As expected, there was also a significant effect as a function of group for the outcome act score, $F(4,205)=7.04, p<0.00003$, $\eta_{\mathrm{p}}^{2}=0.35$ with each treatment group (Groups 1-4) having significantly higher scores than the Control (baseline group), all $p s \leq 0.001$.

To provide a further statistical probe, we also conducted planned comparisons among the four treatment groups (Groups 1-4) to assess the effects of prior self-experience and pedagogical cues. A 2(Prior self-experience: yes vs. no) $\times 2$ (Pedagogical cues: yes vs. no) ANOVA was conducted on each dependent measure. For the target act score, as predicted (Williamson et al., 2008; Wood et al., 2013), there was a significant main effect of prior self-experience, $F(1,164)=6.34, p=0.013, \eta_{\mathrm{P}}^{2}=0.037$ : When children did not have prior self-experience with the objects $(M=9.81)$ they produced significantly more of the novel target acts than when they had prior experience handling the objects $(M=8.93)$, (Figure 3$)$. There was no significant main effect of pedagogical cues, $F(1,164)=1.85, p=0.175$, and there was no prior experience $\times$ pedagogical cues interaction, $F(1,164)=1.50, p=0.222$.

For the serial order score, there was also a significant main effect of prior self-experience, $F(1,164)=13.59, p<0.0004$, $\eta_{\mathrm{P}}^{2}=0.077$, showing that children who had no prior selfexperience $(M=3.11)$ were significantly more likely to imitate the serial ordering of the behaviors they observed than children with prior self-experience $(M=2.45)$ (Figure 3$)$. There was no significant main effect of pedagogical cues, $F(1,164)=0.36$, $p=0.547$, and no prior experience $\times$ pedagogical cues interaction, $F(1,164)=0.76, p=0.385$.

For the outcome act score, there was no significant main effect of prior self-experience, $F(1,164)=2.15$. $p=0.145$ (Figure 3), and also no significant main effect of pedagogical cues, $F(1,164)=0.66, p=0.417$, and no prior experience $\times$ pedagogical cues interaction, $F(1,164)=0.11, p=0.745$. These results are informative when considered in the context of the larger pattern of results. More specifically, they show that even though children re-enacted significantly more of the causal outcome acts when they saw them modeled in the four Demonstration groups (Groups 1-4) than they did in the Control (baseline) group (see Table 2 and statistical results reported above), the children produced these causal outcome acts regardless of whether or not they had prior experience or pedagogical cues (possibly because these acts led to a physical outcome or reward of finding the toy). However, there was significant modulation of imitation as a function of prior self-experience for imitation of the non-causal, novel target acts in the same children in the same experiment. These novel acts did not have to be performed by the child to reach the instrumental end of finding the toy.

\section{DISCUSSION}

The current study extends efforts to understand the nature and scope of imitation in children who are not from Western, educated, industrialized, rich democratic societies. This effort is partially motivated by the desire to determine which aspects of imitation may be more culturally invariant and which are more variable and dependent on cultural context. A range of topics within social learning, using different paradigms, have been tested using non-Western samples (e.g., Itakura et al., 2008; Nielsen and Tomaselli, 2010; Nielsen et al., 2014; Berl and Hewlett, 2015; DiYanni et al., 2015; Wang et al., 2015; Clegg and Legare, 2016; Corriveau et al., 2017; Taniguchi and Sanefuji, 2017; Hoehl et al., 2019; Li et al., 2019). However, little research has investigated the imitation of preschool children born and raised within China, a country that more than 1 billion people (for exceptions see Wang et al., 2015; Li et al., 2019). Children raised in China tend to be socialized in ways that are distinct from Western cultures, and also from many of the non-Western cultures tested in the studies referred to above. The present work is the first to systematically test how two prominent factors reported to modulate imitation in Western children-pedagogical cues and prior self-experience-influence children's imitation in China.

One aim of the current study was to investigate whether preschool Chinese children's imitation of novel acts varied as a function prior self-experience. We found that such experience significantly influenced high-fidelity imitation of specific novel target acts demonstrated by the adult. These finding are in line with studies of self-experience involving Western children (Williamson et al., 2008; Williamson and Meltzoff, 2011; Wood et al., 2013; Schleihauf et al., 2018).

We offer the speculation, based on these results and extant theory, that the influence of prior self-experience may be (relatively) culturally invariant, although more research is needed across a wider range of cultures. We theorize that invariance across cultures makes adaptive sense for the prior experience factor, because it draws on what the child extracts from manipulating the object on their own-the consequences of selfactions and self-agency, which is part of play behavior across the world. Through exploring the object themselves, children often develop their own successful routines, procedures, and conceptions of how to use the object. In certain contexts this can diminish the high-fidelity imitation of novel, meaningless, and irrelevant acts demonstrated by others. This pattern of findings can be linked to theories of education. Children are agents who acquire knowledge by self-directed exploration the physical and social world. The value of young children's play and joyful feelings self-agency is often emphasized in early education (Bruce, 2018; Delafield-Butt, 2018; Trevarthen, 2018). Some psychologists and educators have suggested that in the preschool classroom, it is conducive to allow children to become more active agents by purposely diminishing the adult's own activity and authority (Montessori, 1966; Trevarthen et al., 2018). The power of play for engendering creative interactions with objects was originally emphasized by Vygotsky (1978) as well as by Piaget (1952, 1962) who contrasted the child's drive for exploration and assimilation (play) with that of accommodation to others 
(imitation). Ultimately, the engine for human development and learning is fueled by both play and imitation. Children need to combine first-person, hands-on experiences (play) and those experiences gained from third-party observation of the acts of other people (imitation). Modern, effective preschool education can strive to foster children's adaptive ability to integrate these activities according to the social, emotional, and cognitive goals and contexts at hand.

Another aim of the current study was to examine the degree to which Chinese children's imitation was influenced by pedagogical cues. Results showed that native Chinese children reproduced the novel target acts at approximately the same levels regardless of whether these acts were demonstrated with or without the support of pedagogical cues. This finding does not fit easily with the predictions from the theory of natural pedagogy (Gergely et al., 2007; Csibra and Gergely, 2009). According to this idea, at least in its strongest form, pedagogical cues indicate to children that the adult is teaching cultural knowledge about how to use the object which may engender, or at least enhance high-fidelity imitation of novel acts. However, native Chinese children did not respond in a significantly different way to demonstrations with pedagogical cues versus seeing those same acts demonstrated without the pedagogical cues. This restricts the scope of theories about pedagogical cues and suggests they may be more applicable to children reared in Western rather than traditional Chinese culture (or perhaps play a greater role in children at a different age than those tested here). Going one step further, the current results align well with emerging findings reporting that young Western children can copy modeled actions when no pedagogical cues are present (Schmidt et al., 2011; Shimpi et al., 2013; Hoehl et al., 2014). It is thus possible that pedagogical cues are not as necessary as a strong view would predict, but may elevate the expression of imitation in Western children under particular circumstances. Further research on Western children varying the age/developmental level and the specific tasks used (Yu and Kushnir, 2014, 2020) may bring further clarity to these issues, but they are beyond the scope of the current research.

The proclivity of human children to imitate novel, non-causal, "meaningless" acts as well as their serial order is noteworthy.
Non-human primates are capable of duplicating outcomes or end-states (such as opening a container to retrieve an edible piece of food, sometimes called "goal emulation"), but they less readily engage in high-fidelity imitation of the arbitrary novel acts and mannerisms of a model when they have no physicalcausal significance or rewarding outcomes (Hoehl et al., 2019; Tomasello, 2019). It has also been reported in children with autism spectrum disorder (ASD) show more deficits in imitating of the specific behaviors and arbitrary mannerisms of adult models than in achieving the demonstrated outcomes or endstates through other means (for a meta-analysis of imitation in children with ASD see Edwards, 2014; see also Toth et al., 2006; Nadel, 2014).

The tendency of typically developing human children to imitate the details of arbitrary novel acts with high fidelity, as shown in the current study, fits hand-in-glove with the uniquely human characteristic of diverse and cumulative culture (Legare, 2017; Meltzoff and Marshall, 2018; Tomasello, 2019). Such highfidelity imitation enables children to acquire complex behaviors that they are unlikely to hit upon by themselves (e.g., Yu and Kushnir, 2014; Subiaul et al., 2015). Moreover, high-fidelity imitation of the serial order of novel acts (also documented here) is especially well-suited for the intergenerational transfer of culturally specific customs and rituals. To enact rituals, one needs to do specific behaviors in the right sequence for it to "count." In Western cultures, the incantation in church must precede the sip of wine-not the reverse. Likewise, in a prominent Chinese Buddhist worship ritual, one chants scriptures, makes a kowtow, and then inserts the incense into the center of the alter. One does not insert the incense first and then make a kowtow.

We established that children not only imitated novel, arbitrary acts, but also that they tended to repeat these acts in the same serial order in which they witnessed them, and they exhibited significantly higher levels of such novel imitation when they did not have prior self-experience with the objects that would have led them to manipulate the objects in other ways. Interestingly, "sacred objects" are often kept quarantined and saved for ritualistic occasions, not usually handled in ways that conflict with the ritual. In adult rituals (and perhaps to a lesser

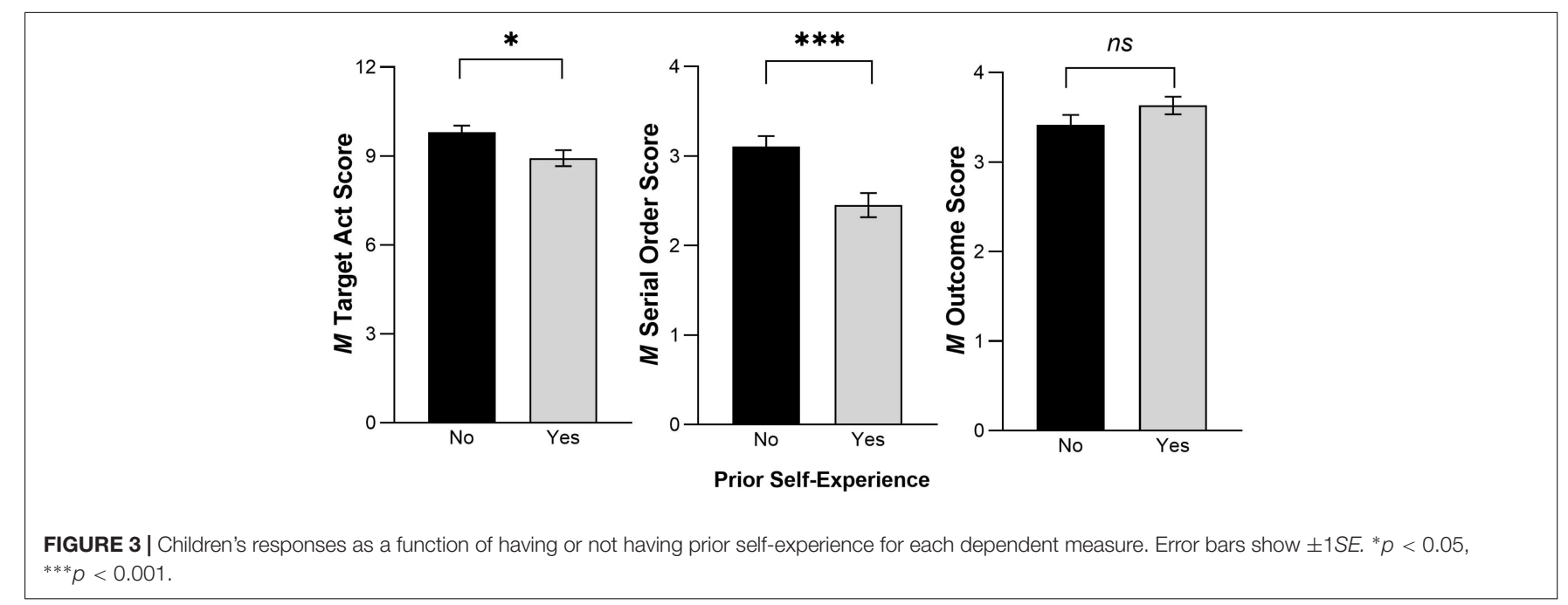


extent the novel acts in this study), the "meaning" of the sequence of witnessed acts does not derive from the fact that they cause an immediate, physically contingent outcome or reward, but from the fact that the whole ritual-including the serial order of the acts (the chanting then the kowtowing then the placing of incense) - takes on social meaning.

Together, these findings suggest that future theoretical effort should be devoted to how children learn both the sociocultural conventional and non-conventional uses of objects by interweaving their observation of others together with their own personal history with the objects and actions. One emerging perspective, dubbed the "socio-materiality" viewpoint (Iannaccone, 2015) has begun this examine this complex interaction between people (self/other), objects, and cultural meanings to assess how they interact in social-cognitive development. This fundamental issue also animated the work of Piaget (1962) and Vygotsky (1978) and is increasingly informing modern perspectives on early education (Master et al., 2017; Trevarthen et al., 2018).

\section{Limitations, Future Directions, and Conclusion}

The current study is not without limitations. First, we tested children using the standard procedure of having the adult model remain present during the child's response period. This is common with studies of 4 -year-old children because it is not so easy to leave them alone and unattended in a room. It is possible that children in China regard the model as an authority figure or teacher who they should conform to (her presence might provide a motivation to perform the act, although she remained present in all of the groups tested, and thus the differences between groups cannot be attributed to this). It would thus be interesting to design future experiments of prior self-experience and pedagogical cues, while experimentally manipulating whether the model did or did not remain present watching the child's actions (for studies on the role of the presence of the experimenter, see e.g., Hanna and Meltzoff, 1993; Klein and Meltzoff, 1999; Repacholi and Meltzoff, 2007; Nielsen and Blank, 2011; Hoehl et al., 2014).

Second, it would be useful to use the identical experimental procedures in both China and the United States. At present, we can only draw loose inferences about (Western) pedagogical cues not having as strong an effect in children born and raised in China as they do in Western cultures. This is because the various studies evaluating pedagogical cues in Western cultures have used different ages, procedures, and/or objects from each other, and so strict cross-cultural comparisons are difficult. Our primary aim was to investigate how these factors influence imitation in China, a country encompassing more than 1 billion people and of interest to theory because of the value placed on group cohesion, harmony, and conformity, and a different pattern of child-rearing practices than Western cultures, which could influence young children's social and "other-directed" behavior (e.g., Barragan et al., 2020). A controlled comparison to Western samples using this same paradigm and age was beyond the scope of this paper.
Third, our inferences are limited to the broad but delimited set of objects and tasks that we tested. We used a range of objects (four) and a range of acts (three novel acts plus one goaldirected causal outcome act), but there are many other different types of demonstrations that are also of interest (e.g., tool affordances; variations in the causal opacity of the acts; reliability, trustworthiness, and efficacy of the model; manipulations that vary the motivation to affiliate with the adult or conform to cultural norms; demonstrations by ingroup vs. outgroup models, etc.). We are not making the claim that the factors explored here are the only factors that modulate preschool imitation. Further research could be conducted that pits prior self-experience and pedagogical cues against one or more of these other foregoing factors, both within and across cultures, to further examine cultural variations in factors that govern childhood imitation of novel acts.

\section{Broader Theoretical Implications About Imitation, Culture, Mind}

Continued research is warranted on factors that modulate preschool children's high-fidelity imitation of novel acts. The diverse and cumulative aspects of human culture-widely celebrated by evolutionary biologists and psychologists (e.g., Henrich and McElreath, 2003; Legare, 2017; Tomasello, 2019)crucially depends on learning novel acts through observation and imitation from others in the cultural milieu (Meltzoff and Marshall, 2018). Importantly, young human children can and do imitate novel acts in situations in which people are not intentionally teaching them. Imitation is a powerful mechanism for the intergenerational transfer of behaviors, skills, customs, and norms, based purely on observation of the acts of others, even in the absence of those people's conscious efforts to teach. Children around the world and in all cultures learn from observing and imitating others; however, what they imitate, who they imitate, and when they imitate is malleable. By further understanding what motivates and modulates imitation, we will enhance our understanding of mind, culture, and social learning.

\section{DATA AVAILABILITY STATEMENT}

The datasets generated for this study are available on request to the corresponding author.

\section{ETHICS STATEMENT}

The studies involving human participants were reviewed and approved by ethics committee of Jiangsu Normal University. Written informed consent to participate in this study was provided by the participants' legal guardian/next of kin.

\section{AUTHOR CONTRIBUTIONS}

ZW and AM conceptualized the experiments. ZW designed the methodology, performed the experiments, and analyzed the data. ZW and AM wrote the manuscript. 


\section{FUNDING}

This research was supported by funding for Humanities and Social Sciences from the Chinese Ministry of Education (18YJC190024) to ZW.

\section{REFERENCES}

Barragan, R. C., Brooks, R., and Meltzoff, A. N. (2020). Altruistic food sharing behavior by human infants after a hunger manipulation. Sci. Rep. 10:1785. doi: 10.1038/s41598-020-58645-9

Berl, R. E. W., and Hewlett, B. S. (2015). Cultural variation in the use of overimitation by the Aka and Ngandu of the Congo Basin. PLoS One 10:e0120180. doi: 10.1371/journal.pone.0120180

Bruce, T. (2018). "The importance of play," in The Child's Curriculum: Working with the Natural Values of Young Children, eds C. Trevarthen, J. Delafield-Butt, and A.-W. Dunlop, (Oxford: Oxford University Press).

Buchsbaum, D., Gopnik, A., Griffiths, T. L., and Shafto, P. (2011). Children's imitation of causal action sequences is influenced by statistical and pedagogical evidence. Cognition 120, 331-340. doi: 10.1016/j.cognition.2010.12.001

Carpenter, M., Call, J., and Tomasello, M. (2002). Understanding "prior intentions" enables two-year-olds to imitatively learn a complex task. Child Dev. 73, 1431-1441. doi: 10.1111/1467-8624.00481

Chang, L., Schwartz, D., Dodge, K. A., and McBride-Chang, C. (2003). Harsh parenting in relation to child emotion regulation and aggression. J. Fam. Psychol. 17, 598-606. doi: 10.1037/0893-3200.17.4.598

Chao, R. K. (1994). Beyond parental control and authoritarian parenting style: understanding Chinese parenting through the cultural notion of training. Child Dev. 65, 1111-1119. doi: 10.1111/j.1467-8624.1994.tb00806.x

Chen, X., Liu, M., Li, B., Cen, G., Chen, H., and Wang, L. (2000). Maternal authoritative and authoritarian attitudes and mother-child interactions and relationships in urban China. Int. J. Behav. Dev. 24, 119-126. doi: 10.1080/ 016502500383557

Clegg, J. M., and Legare, C. H. (2016). A cross-cultural comparison of children's imitative flexibility. Dev. Psychol. 52, 1435-1444. doi: 10.1037/dev0000131

Corriveau, K. H., DiYanni, C. J., Clegg, J. M., Min, G., Chin, J., and Nasrini, J. (2017). Cultural differences in the imitation and transmission of inefficient actions. J. Exp. Child Psychol. 161, 1-18. doi: 10.1016/j.jecp.2017.03.002

Csibra, G., and Gergely, G. (2009). Natural pedagogy. Trends Cogn. Sci. 13, 148-153. doi: 10.1016/j.tics.2009.01.005

Delafield-Butt, J. (2018). "The emotional and embodied nature of human understanding: sharing narratives of meaning," in The Child's Curriculum: Working with the Natural Values of Young Children, eds C. Trevarthen, J. Delafield-Butt, and A.-W. Dunlop, (Oxford: Oxford University Press).

DiYanni, C., and Kelemen, D. (2008). Using a bad tool with good intention: young children's imitation of adults' questionable choices. J. Exp. Child Psychol. 101, 241-261. doi: 10.1016/J.Jecp/2008.05.002

DiYanni, C. J., Corriveau, K. H., Kurkul, K., Nasrini, J., and Nini, D. (2015). The role of consensus and culture in children's imitation of inefficient actions. J. Exp. Child Psychol. 137, 99-110. doi: 10.1016/j.jecp.2015.04.004

Edwards, L. A. (2014). A meta-analysis of imitation abilities in individuals with autism spectrum disorders. Autism Res. 7, 363-380. doi: 10.1002/aur.1379

Gergely, G., Egyed, K., and Király, I. (2007). On pedagogy. Dev. Sci. 10, 139-146. doi: $10.1111 / j .1467-7687.2007 .00576 . x$

Gonsiorowski, A., Williamson, R. A., and Robins, D. L. (2016). Brief report: imitation of object-directed acts in young children with autism spectrum disorders. J. Autism Dev. Disord. 46, 691-697. doi: 10.1007/s10803-015-2596-1

Hanna, E., and Meltzoff, A. N. (1993). Peer imitation by toddlers in laboratory, home, and day-care contexts: implications for social learning and memory. Dev. Psychol. 29, 701-710. doi: 10.1037/0012-1649.29.4.701

Henrich, J., Heine, S. J., and Norenzayan, A. (2010). The weirdest people in the world? Behav. Brain Sci. 33, 61-135. doi: 10.1017/S0140525X0999152X

Henrich, J., and McElreath, R. (2003). The evolution of cultural evolution. Evol. Anthropol. 12, 123-135. doi: 10.1002/evan.10110

\section{ACKNOWLEDGMENTS}

We thank Francys Subiaul, Yue Yu, and Rechele Brooks for useful feedback on an earlier draft and the participating children and families for their cooperation.

Hoehl, S., Keupp, S., Schleihauf, H., McGuigan, N., Buttelmann, D., and Whiten, A. (2019). 'Over-imitation': a review and appraisal of a decade of research. Dev. Rev. 51, 90-108. doi: 10.1016/j.dr.2018.12.002

Hoehl, S., Zettersten, M., Schleihauf, H., Grätz, S., and Pauen, S. (2014). The role of social interaction and pedagogical cues for eliciting and reducing overimitation in preschoolers. J. Exp. Child Psychol. 122, 122-133. doi: 10.1016/j.jecp.2013. 12.012

Iannaccone, A. (2015). Materiality and educational psychology. Paper Presented at the Symposium on Materiality and Human Development conducted at the meeting of the 16th biennial conference of International Society for Theoretical Psychology, Coventry.

Itakura, S., Ishida, H., Kanda, T., Shimada, Y., Ishiguro, H., and Lee, K. (2008). How to build an intentional android: infants' imitation of a robot's goal-directed actions. Infancy 13, 519-532. doi: 10.1080/15250000802329503

Kenward, B. (2012). Over-imitating preschoolers believe unnecessary actions are normative and enforce their performance by a third party. J. Exp. Child Psychol. 112, 195-207. doi: 10.1016/j.jecp.2012.02.006

Kenward, B., Karlsson, M., and Persson, J. (2011). Over-imitation is better explained by norm learning than by distorted causal learning. Proc. R. Soc. B Biol. Sci. 278, 1239-1246. doi: 10.1098/rspb.2010.1399

Keupp, S., Behne, T., and Rakoczy, H. (2013). Why do children overimitate? Normativity is crucial. J. Exp. Child Psychol. 116, 392-406. doi: 10.1016/j.jecp. 2013.07.002

Király, I., Csibra, G., and Gergely, G. (2013). Beyond rational imitation: learning arbitrary means actions from communicative demonstrations. J. Exp. Child Psychol. 116, 471-486. doi: 10.1016/j.jecp.2012.12.003

Klein, P. J., and Meltzoff, A. N. (1999). Long-term memory, forgetting, and deferred imitation in 12-month-old infants. Dev. Sci. 2, 102-113. doi: 10.1111/14677687.00060

Legare, C. H. (2017). Cumulative cultural learning: development and diversity. Proc. Natl. Acad. Sci. U.S.A. 114, 7877-7883. doi: 10.1073/pnas.1620743114

Legare, C. H., and Nielsen, M. (2015). Imitation and innovation: the dual engines of cultural learning. Trends Cogn. Sci. 19, 688-699. doi: 10.1016/j.tics.2015.08.005

Li, Y., Liao, Y., Cheng, Y., and He, J. (2019). Group conquers efficacy: preschoolers' imitation under conflict between minimal group membership and behavior efficacy. PLoS One 14:e0223101. doi: 10.1371/journal.pone.0223101

Loucks, J., Mutschler, C., and Meltzoff, A. N. (2017). Children's representation and imitation of events: how goal organization influences 3-year-old children's memory for action sequences. Cogn. Sci. 41, 1904-1933. doi: 10.1111/cogs. 12446

Lyons, D. E., Damrosch, D. H., Lin, J. K., Macris, D. M., and Keil, F. C. (2011). The scope and limits of overimitation in the transmission of artefact culture. Philos. Trans. R. Soc. B Biol. Sci. 366, 1158-1167. doi: 10.1098/rstb.2010.0335

Lyons, D. E., Young, A. G., and Keil, F. C. (2007). The hidden structure of overimitation. Proc. Natl. Acad. Sci. U.S.A. 104, 19751-19756. doi: 10.1073/ pnas.0704452104

Master, A., Cheryan, S., Moscatelli, A., and Meltzoff, A. N. (2017). Programming experience promotes higher STEM motivation among first-grade girls. J. Exp. Child Psychol. 160, 92-106. doi: 10.1016/j.jecp.2017.03.013

McGuigan, N., and Robertson, S. (2015). The influence of peers on the tendency of 3- and 4-year-old children to over-imitate. J. Exp. Child Psychol. 136, 42-54. doi: 10.1016/j.jecp.2015.03.004

McGuigan, N., Whiten, A., Flynn, E., and Horner, V. (2007). Imitation of causally opaque versus causally transparent tool use by 3 -and 5-year-old children. Cogn. Dev. 22, 353-364. doi: 10.1016/j.cogdev.2007.01.001

Meltzoff, A. N. (1988a). Infant imitation after a 1-week delay: long-term memory for novel acts and multiple stimuli. Dev. Psychol. 24, 470-476. doi: 10.1037/ 0012-1649.24.4.47 
Meltzoff, A. N. (1988b). “The human infant as Homo imitans," in Social Learning: Psychological and Biological Perspectives, eds T. R. Zentall, and B. G. Galef, (Hillsdale, NJ: Erlbaum), 319-341.

Meltzoff, A. N., Kuhl, P. K., Movellan, J., and Sejnowski, T. J. (2009). Foundations for a new science of learning. Science 325, 284-288. doi: 10.1126/science. 1175626

Meltzoff, A. N., and Marshall, P. J. (2018). Human infant imitation as a social survival circuit. Curr. Opin. Behav. Sci. 24, 130-136. doi: 10.1016/j.cobeha.2018. 09.006

Meltzoff, A. N., and Williamson, R. A. (2013). "Imitation: social, cognitive, and theoretical perspectives," in The Oxford Handbook of Developmental Psychology, Vol. 1, ed. P. R. Zelazo, (New York, NY: Oxford University Press), 651-682.

Montessori, M. (1966). The Secret of Childhood. New York, NY: Ballantine Books.

Nadel, J. (2014). How Imitation Boosts Development: In Infancy and Autism Spectrum Disorder. Oxford: Oxford University Press.

Nielsen, M. (2006). Copying actions and copying outcomes: social learning through the second year. Dev. Psychol. 42, 555-565. doi: 10.1037/0012-1649.42.3.555

Nielsen, M., and Blank, C. (2011). Imitation in young children: when who gets copied is more important than what gets copied. Dev. Psychol. 47, 1050-1053. doi: $10.1037 / \mathrm{a} 0023866$

Nielsen, M., Moore, C., and Mohamedally, J. (2012). Young children overimitate in third-party contexts. J. Exp. Child Psychol. 112, 73-83. doi: 10.1016/j.jecp.2012. 01.001

Nielsen, M., Mushin, I., Tomaselli, K., and Whiten, A. (2014). Where culture takes hold: "Overimitation" and its flexible deployment in Western. Aboriginal, and Bushmen children. Child Dev. 85, 2169-2184. doi: 10.1111/cdev.12265

Nielsen, M., and Tomaselli, K. (2010). Overimitation in Kalahari Bushman children and the origins of human cultural cognition. Psychol. Sci. 21, 729-736. doi: $10.1177 / 0956797610368808$

Over, H., and Carpenter, M. (2012). Putting the social into social learning: explaining both selectivity and fidelity in children's copying behavior. J. Comp. Psychol. 126, 182-192. doi: 10.1037/a0024555

Piaget, J. (1952). The Origins of Intelligence in Children. New York, NY: International Universities Press.

Piaget, J. (1962). Play, Dreams and Imitation in Childhood. New York, NY: Norton.

Repacholi, B. M., and Meltzoff, A. N. (2007). Emotional eavesdropping: infants selectively respond to indirect emotional signals. Child Dev. 78, 503-521. doi: 10.1111/j.1467-8624.2007.01012.x

Rossano, M. J. (2012). The essential role of ritual in the transmission and reinforcement of social norms. Psychol. Bull. 138, 529-549. doi: 10.1037/ a0027038

Schleihauf, H., Graetz, S., Pauen, S., and Hoehl, S. (2018). Contrasting social and cognitive accounts on overimitation: the role of causal transparency and prior experiences. Child Dev. 89, 1039-1055. doi: 10.1111/cdev.12780

Schleihauf, H., Pauen, S., and Hoehl, S. (2019). Minimal group formation influences on over-imitation. Cogn. Dev. 50, 222-236. doi: 10.1016/j.cogdev. 2019.04.004

Schmidt, M. F. H., Rakoczy, H., and Tomasello, M. (2011). Young children attribute normativity to novel actions without pedagogy or normative language. Dev. Sci. 14, 530-539. doi: 10.1111/j.1467-7687.2010.01000.x

Shek, D. T. L. (2002). Family functioning and psychological well-being, school adjustment, and problem behavior in Chinese adolescents with and without economic disadvantage. J. Genet. Psychol. 163, 497-502. doi: 10.1080/ 00221320209598698

Shimpi, P. M., Akhtar, N., and Moore, C. (2013). Toddlers' imitative learning in interactive and observational contexts: the role of age and familiarity of the model. J. Exp. Child Psychol. 116, 309-323. doi: 10.1016/j.jecp.2013. 06.008

Skinner, A. L., Meltzoff, A. N., and Olson, K. R. (2017). "Catching” social bias: exposure to biased nonverbal signals creates social bias in preschool children. Psychol. Sci. 28, 216-224. doi: 10.1177/0956797616678930

Skinner, A. S., Olson, K. R., and Meltzoff, A. N. (2019). Acquiring group bias: observing other people's nonverbal signals can create social group biases. J. Pers. Soc. Psychol. [Epub ahead of print].
Subiaul, F., Patterson, E. M., Schilder, B., Renner, E., and Barr, R. (2015). Becoming a high-fidelity-super-imitator: what are the contributions of social and individual learning? Dev. Sci. 18, 1025-1035. doi: 10.1111/desc.12276

Taniguchi, Y., and Sanefuji, W. (2017). The boundaries of overimitation in preschool children: effects of target and tool use on imitation of irrelevant actions. J. Exp. Child Psychol. 159, 83-95. doi: 10.1016/j.jecp.2017.01.014

Tomasello, M. (2019). Becoming Human: A Theory of Ontogeny. Cambridge, MA: Harvard University Press.

Tomasello, M., Kruger, A. C., and Ratner, H. H. (1993). Cultural learning. Behav. Brain Sci. 16, 495-552. doi: 10.1017/S0140525X0003123X

Toth, K., Munson, J., Meltzoff, A. N., and Dawson, G. (2006). Early predictors of communication development in young children with autism spectrum disorder: joint attention, imitation, and toy play. J. Autism Dev. Disord. 36, 993-1005. doi: 10.1007/s10803-006-0137-7

Trevarthen, C. (2018). "Why young children give to our learning," in The Child's Curriculum: Working with the Natural Values of Young Children, eds C. Trevarthen, J. Delafield-Butt, and A.-W. Dunlop, (Oxford: Oxford University Press).

Trevarthen, C., Delafield-Butt, J., and Dunlop, A.-W. (2018). The Child's Curriculum: Working with the Natural Values of Young Children. Oxford: Oxford University Press.

Vygotsky, L. S. (1978). Mind in Society: The Development of Higher Psychological Processes. Cambridge, MA: Harvard University Press.

Wang, Z., Williamson, R. A., and Meltzoff, A. N. (2015). Imitation as a mechanism in cognitive development: a cross-cultural investigation of 4-year-old children's rule learning. Front. Psychol. 6:562. doi: 10.3389/fpsyg.2015.00562

Want, S. C., and Harris, P. L. (2001). Learning from other people's mistakes: causal understanding in learning to use a tool. Child Dev. 72, 431-443. doi: $10.1111 / 1467-8624.00288$

Wilks, M., Kirby, J., and Nielsen, M. (2019). Developmental changes in young children's willingness to copy the antisocial actions of ingroup members in a minimal group context. Dev. Psychol. 55, 709-721. doi: 10.1037/dev0000667

Williamson, R. A., and Meltzoff, A. N. (2011). Own and others' prior experiences influence children's imitation of causal acts. Cogn. Dev. 26, 260-268. doi: 10. 1016/j.cogdev.2011.04.002

Williamson, R. A., Meltzoff, A. N., and Markman, E. M. (2008). Prior experiences and perceived efficacy influence 3-year-olds' imitation. Dev. Psychol. 44, 275285. doi: 10.1037/0012-1649.44.1.275

Wood, L. A., Kendal, R. L., and Flynn, E. G. (2013). Copy me or copy you? The effect of prior experience on social learning. Cognition 127, 203-213. doi: 10.1016/j.cognition.2013.01.002

Xu, Y., Zhang, L., and Hee, P. (2014). "Parenting practices and shyness in Chinese children," in Parenting Across Cultures: Childrearing, Motherhood and Fatherhood in Non-Western Cultures, ed. H. Selin, (Dordrecht: Springer), 13-24. doi: 10.1007/978-94-007-7503-9_2

Yu, Y., and Kushnir, T. (2014). Social context effects in 2- and 4-year-olds' selective versus faithful imitation. Dev. Psychol. 50, 922-933. doi: 10.1037/a0034242

Yu, Y., and Kushnir, T. (2020). The ontogeny of cumulative culture: individual toddlers vary in faithful imitation and goal emulation. Dev. Sci. 23:e12682. doi: $10.1111 /$ desc. 12862

Zhu, J., and Zhang, J. (2008). Contemporary trends and developments in early childhood education in China. Early Years 28, 173-182. doi: 10.1080/ 09575140802163584

Conflict of Interest: The authors declare that the research was conducted in the absence of any commercial or financial relationships that could be construed as a potential conflict of interest.

Copyright (c) 2020 Wang and Meltzoff. This is an open-access article distributed under the terms of the Creative Commons Attribution License (CC BY). The use, distribution or reproduction in other forums is permitted, provided the original author(s) and the copyright owner(s) are credited and that the original publication in this journal is cited, in accordance with accepted academic practice. No use, distribution or reproduction is permitted which does not comply with these terms. 


\title{
The Role of Common Ground on Object Use in Shaping the Function of Infants' Social Gaze
}

\author{
Nevena Dimitrova* \\ Haute École de travail social et de la santé (HETSL), Haute École Spécialisée de Suisse Occidentale (HES-SO), Lausanne, \\ Switzerland
}

Although infants' social gaze has specific communicative functions, it remains unclear what they are. In this conceptual analysis paper, we provide a theoretical framework for the study of the functional aspects of eye gaze in early childhood. We argue that studying the communicative functions of infants' eye gaze involves three premises: the centrality of the object, the importance of common ground on object use, and the role of parental interpretations. The ability to communicate intentionally begins when infants start referring to external objects. Beyond dyadic - infant-parent - emotional sharing,

OPEN ACCESS

Edited by:

Giulia Savarese,

University of Salerno, Italy

Reviewed by:

Mitsuhiko Ishikawa,

Kyoto University, Japan

Livio Provenzi,

Neurological Institute Foundation

Casimiro Mondino (IRCCS), Italy

*Correspondence:

Nevena Dimitrova

Nevena.Dimitrova@hetsl.ch

Specialty section: This article was submitted to

Developmental Psychology, a section of the journal

Frontiers in Psychology

Received: 08 November 2019

Accepted: 16 March 2020

Published: 16 April 2020

Citation:

Dimitrova N (2020) The Role of Common Ground on Object Use in Shaping the Function of Infants' Social Gaze. Front. Psychol. 11:619. doi: 10.3389/fpsyg.2020.00619 infant social gaze within the infant-parent-object triad becomes an increasingly complex communicative modality. As the predominant type of communicative referents in infancy, objects are thus central to early communication. Although they have affordances, objects are used in conventional ways shared between users (i.e., common ground). Parents transmit to infants the socio-cultural use of objects, which infants progressively learn and master. Accordingly, we argue that within early triadic interactions, the communicative function of infants' eye gaze is shaped by the knowledge that the infant and the parent share on the socio-cultural use of the referent (i.e., the object). Importantly, before young children develop their ability to convey clear communicative functions, including with eye gaze, the interpretations and responses that parents provide to infants' early communicative acts play a major role. Relying on these premises, we argue that when referring to objects for which the infant and the parent share common ground, the function of the infant's social gaze becomes communicatively meaningful for the parent. The knowledge on the communicative referent (i.e., the object) shared between the infant and the parent thus shapes the course of communicative behavior, constitutes and reflects the interactive function of gaze, and cues parents into tailoring their communicative response according to the infant's developmental needs. Through this theoretical framework for the study of the communicative function of infant eye gaze, an emphasis is put on the key role that socio-materiality plays in early communicative development.

Keywords: eye gaze, communicative function, infancy, parent-child interaction, socio-materiality, object use, early development 


\section{INTRODUCTION}

As social beings, infants seek social interaction from the very beginning of life. They produce a myriad of communicative behaviors - first without intention, then intentionally - in order to communicate with their social partners (Rochat, 2001). Infants do so in order to express their internal state, to share emotional experiences, and later on, to influence their communicative partners. Among the earliest communicative means infants use is eye gaze (Volkmar and Mayes, 1990). Progressively, infants come to produce other (smiles, cries, vocalizations) and more complex (postures, gestures) communicative means (Adamson, 1996); however, eye gaze remains a principal component of infants' earliest communicative acts. While the question of communicative intentionality in infancy has been the subject of a longsome debate, there are different established criteria allowing characterization of an infant's communicative bid as intentional. Importantly, a key criterion for the earliest manifestations of intentional communication is that an infant's eye gaze is oriented toward the communicative partner (Bruner, 1973; Bates, 1979; Franco and Butterworth, 1996). Despite the centrality of an infant's eye gaze in early communication development, the majority of studies have primarily examined how eye gaze accompanies other communicative means (vocalizations, gestures). Thus, to date, it remains unclear what the communicative functions of infant eye gazing toward a communicative partner are.

\section{Communicative Functions}

Because of our subjective experience and fragmentary knowledge of the world, communication is ambiguous, imprecise, and messy (Wittgenstein, 1962). The means that we use to communicate do not always convey a literal meaning; in fact, they are essentially equivocal. Linguists have examined this phenomenon by introducing the term "communicative act" - namely, the action (or set of actions) that a speaker accomplishes by producing a communicative bid. The Speech Act theory (Austin, 1962; Searle, 1969, 1975) supported the idea that utterances can have different levels of meaning at which actions are accomplished (Casillas and Hilbrink, 2020). It thus differentiated three types of acts: perlocutionary acts as the effect of having made an utterance; illocutionary acts as the effect on the listener which the speaker intended; and locutionary acts as the construction of propositions and uttering sounds.

Developmentally, there is a progression from perlocutionary to illocutionary and locutionary acts (Bates, 1979). Specifically, with their earliest communicative acts, it is unclear whether infants use them intentionally or not. Regardless, parents readily provide interpretations of the infant's vocal and embodied activities as potentially meaningful communicative acts (perlocutionary acts; Bates, 1976). Around 10 months of age, infants begin to intentionally use a conventional signal in order to carry out some socially recognized functions (illocutionary acts). Through linguistic and/or embodied means (such as eye gaze, gesture), they start communicating the force or intent of a request, a command, a promise, or other social actions. When, by 12 months, children begin to use words with a referential value, they enter the stage of locutionary acts (Harding and Golinkoff, 1979).

In studies on early language development, researchers have primarily focused on perlocutionary and illocutionary acts. It is through the pioneering work of Elisabeth Bates on gesture communication that early communication development shifted from studying the form of acts infants use to communicate to the function of such communicative acts (Bates et al., 1975).

\section{Perlocutionary Acts}

In the first few months of life, infants produce a host of behaviors such as eye gaze, cries, smiles, and vocalizations that, at first, are not considered as being intentional but which lay the foundations for the development of communication and language (Adamson, 1996; Rochat, 2001). Infants produce such behaviors in the presence of another person, usually a parent, and a key feature of the response of parents is that they treat these behaviors as intentional and communicatively meaningful (see the concept of mind-mindedness offered by Meins, 1999). Thus, to a strident vocalization by a 6 -month-old infant, a parent can respond, "You are not happy, are you?" Here, although it is unclear whether the infant intended to communicate dissatisfaction, the parent reacts as if he or she did. Bates et al. (1975) characterized such early behaviors as perlocutionary communication in that they often provide an effect on the parent.

\section{Illocutionary Acts}

Although her work spread across a large variety of topics, Bates' main contributions related to the illocutionary force of infants' early communication. Relying on Searle's taxonomy of illocutionary acts (Searle, 1975; Searle and Vanderveken, 1985), Bates and her colleagues focused on performatives (Bates et al., 1979). Starting from the premise that infants and young children in the illocutionary stage (as early as 10 months of age) use non-verbal acts intentionally, two communicative functions were described: the proto-imperative and proto-declarative ones (Bates et al., 1975). The proto-imperative function relates to the child's intentional use of the listener as an agent or tool in achieving some end. For example, a child might request a toy by looking fixedly at the parent's face while extending his or her arm toward the toy. The proto-declarative function relates to the young child's preverbal effort to direct the parent's attention to some event or object in the world. An example is a child enthusiastic to share his or her interest with his or her parent by pointing to an interesting event, such as a clown riding a unicycle.

Beyond presenting perlocutionary and illocutionary acts in a separate way, it is fundamental to highlight that there is a clear developmental continuum from the first to the second. Specifically, it is primarily through the parental responses to infants' earliest communicative acts (along with infants' cognitive capacity for mean-end differentiation, linked to Piaget's coordination of the secondary circular reactions substage of the sensorimotor stage; Dimitrova, 2013) that infants learn that their 
initial eye gaze, cries, smiles, vocalizations, and such can be actually used as a means to elicit a response from the parent (Bornstein et al., 1999).

\section{Communicative Functions of Eye Gaze}

In humans, mutual gaze provides a foundation of communication and social interaction (Kendon, 1967, 1990; Kleinke, 1986; Csibra and Gergely, 2006; Rossano, 2013). Mutual gaze is an ostensive signal allowing establishment or re-establishment of a communicative link between two people (Csibra, 2010). In adult interaction, Patterson (1983)'s sequential model (1983) described the different functions of eye gaze including providing information, regulating interaction, exercising social control, etc.

In infancy, from the first weeks after birth, infants spend a significant amount of alert time in mutual gaze with a parent, and the large majority of early interactions are based on an infant's interest in faces and mutual gaze (Volkmar, 1987). By 6 months of age, infants are equally likely to initiate an interaction both by gazing at their parent as well as by responding to gaze (Kaye and Fogel, 1980). These early interactions require mutual gaze and the interpersonal coordination of gaze. However, despite the fact that gaze is one of the fundamental resources organizing social interchanges and serves various functions in human interaction, there is a lack of studies examining when and how infants use eye gaze in order to communicate.

In the first 2 years of life, gaze serves various functions including engaging in social-communicative transactions and conveying non-verbal cues for social interaction (Volkmar and Mayes, 1990). First, infants are primarily absorbed by dyadic interactions with a parent. The functions of eye gaze during the first half of the first year are mainly related to emotional connectedness and sharing of emotional states. When they are engaged with a person, infants' attention seems confined to the process of interaction itself. Thus, while young infants participate in finely tuned exchanges of affect, the system of communication is essentially expressive (Brazelton et al., 1974; Stem, 1974; Tronick et al., 1979). In the second half of the first year, infants' interactions become gradually triadic as objectfocused attention becomes embedded in social contexts (Werner and Kaplan, 1963; Trevarthen and Hubley, 1978; Bakeman and Adamson, 1984). Infants begin to switch their gaze back and forth between caregiver and object (Newson and Newson, 1975). Such gaze coordination indicates that infants are actively attempting to share attention to something external to the social interaction, establishing that object or event as the focus of joint concern (Murphy and Messer, 1977; Leung and Rheingold, 1981).

There are two notable exceptions of studies examining the function of eye gaze in early development. The first one includes the concept of social referencing, namely, situations in which an infant actively seeks - most commonly by gazing - an adult's emotional expression to help interpret an ambiguous situation (see work on the Still Face procedure; Campos, 1983; Klinnert et al., 1983). The function of the infant's eye gaze in social referencing might be characterized as social signaling, namely, the infant signals to the parent an emotional distress and seeks for cues in order to figure out how to act in an uncertain situation. Thus, while social referencing is a powerful communicative behavior during infancy, it is confined to the expression and exchange around (mostly negative) emotional states. The second exception of available studies on the function of gaze relates to infants' capacity for gaze following (for an overview, see Flom et al., 2007). When infants follow the direction of attention of another person shifting to different features of the environment (i.e., attention-following) or when infants follow the direction of attention of another person toward a referent (i.e., referential gaze following; Scaife and Bruner, 1975; Butterworth and Jarrett, 1991), they gradually learn how another person's attention can differ from their own. Although these abilities play a critical role in social learning, communication, and mental-state inferences (Argyle and Cook, 1976; Deák et al., 2008; Brooks and Meltzoff, 2014), they relate to an infant's ability to respond to - but not to initiate - social interaction.

\section{Common Ground}

How does a communicative partner access the intended meaning if it is not explicitly encoded in the communicative act? Since communication takes many forms and is often expressed indirectly, addressees must either rely on convention or infer what the speaker means from other available evidence. Theoretical accounts have pointed out that successful communication requires that partners share common ground about the referent in order to mutually access one another's intention and thus determine the meaning of a given communicative act (e.g., Lewis, 1969; Schiffer, 1972; Stalnaker, 1978; Bruner, 1983; Tomasello, 1992, 2003; Clark, 1996). Common ground is a pool of experiences, knowledge, and meanings shared between communicative partners.

As Tomasello (2008) puts it, common ground is everything that we know and we know that we both know, also referred as third-order mentality. This aspect is particularly important for successful communication: producing a communicative act necessitates that one knows that the other will understand him or her based on the fact that the other knows that they both know what one means (e.g., I know what "cat" means, I know that you know what "cat" means, and I expect that you know that I know what "cat" means). The intention of a produced communicative act is thus being anchored in a common base of understanding shared between communicative partners. In social interaction, the already existing common ground is continuously being shaped and re-shaped by the participants' verbal and embodied activities. Thus, a shared perceptual common ground (Clark, 1996) - including noticeable (visible, hearable, tangible) objects emerges in real time and becomes mutually known to the participants through embodied and/or verbal acts of attentionsharing (Stukenbrock, 2018; Stukenbrock and Dao, 2019).

Concerning the nature of the content at stake, two general types of common ground about the communicative referent have been described (Clark, 1996; Tomasello, 2008). The first type is called perceptual co-presence, and it refers to the shared knowledge concerning elements from the immediate context of interaction. For example, when you are looking at a street performer and a person next to you says "It is amazing," you would know what that person refers to and what he or she means relying on what you were both looking at (i.e., perceptual 
co-presence). The second type of common ground is called conceptual common ground and relates to a broader range of conventions such as norms and values that are shared in a given culture, society, or group. For example, if a person asks you if you know what time it is, you know that you are supposed to tell what time it is (and not merely answer "Yes, I know") because you rely on conceptual common ground concerning interpersonal communication - in this example, formulation of polite requests.

Considering the very first communicative dynamics between an infant and a parent, it is crucial to understand how infants come to produce intentional communicative acts. Accordingly, a main question is how do infants come to share common ground with parents? Despite the importance of common ground in communication, little is known about the way infants construct and share meanings with their communicative partners. Common ground in infancy has been addressed empirically in a handful of experimental studies on perceptual co-presence in early communication (Ganea and Saylor, 2007; Moll et al., 2008; Liebal et al., 2009). Overall, these studies found that having previously shared a visual experience with a parent and a particular object helps infants as young as 14 months of age to disambiguate a parent's pointing gestures in order to respond appropriately. Beyond perceptual co-presence, how does conceptual knowledge about the object shared between the young child and the parent shape the function of children's early communicative acts?

\section{Framework for Studying Eye Gaze Functions}

The existing evidence highlights that infants' gaze toward a parent is a major meaningful behavior within socialcommunicative interactions. However, to date, it remains unclear what communicative functions eye gaze serves in the early stages of development. In this conceptual analysis, we provide a theoretical framework for the study of the functional aspects of eye gaze in early childhood. This theoretical framework relies on three intertwined premises: (1) the centrality of the object, (2) the importance of common ground on object use, and (3) the role of parental interpretations.

\section{Centrality of the Object}

An essential component of the developmental progression of infants' abilities to communicate is the capacity to integrate an external object or event into the communicative exchange with another person (i.e., secondary subjectivity, Trevarthen and Hubley, 1978). It is precisely within such triadic interactions that infants begin to refer to the external world, primarily by referring to everyday objects from their immediate environment (Werner and Kaplan, 1963; Adamson, 1996), in order to convey requests (mainly regarding objects) or to obtain the parent's attention (usually toward objects; Bates et al., 1979). Both of these performatives are first constructed on the plane of embodied action, namely, by manipulating objects rather than by formulating propositions. The centrality of the object has been emphasized in several processes of language acquisition and development such as the establishment of reference (e.g., Bruner, 1974), sharing joint attention (e.g., Adamson, 1996;
Adamson and Dimitrova, 2014), the production of children's first communicative gestures (i.e., pointing, e.g., Tomasello, 2008), as well as children's first words (see Golinkoff et al., 1994). As genuine catalysts of communication between a parent and a child, objects are central to the early development of communication. In the theoretical framework that we provide, we argue that a meaningful analysis of the communicative functions of an infants' eye gaze should consider the centrality of objects within triadic - parent-infant-object - early interactions (first premise).

\section{Importance of Common Ground on Object Use}

Objects have specific physical properties, such as size, shape, weight, color, softness, etc. Manufacturers specify the physical properties of objects, which provide for a certain amount of affordances (Gibson, 1979). However, the ways objects are used depend not only on their physical properties but on cultural conventions as well (Dimitrova, 2010, 2014). For example, while chopsticks afford a large number of uses (e.g., drumsticks, hairstyle accessory, gardening tools), there is a conventional type of use that is agreed upon and shared culturally. As adults, we master the conventional uses of everyday objects in our cultural environment (and we constantly adapt to the uses of novel objects). Infants, however, do not master such conventions even for simple objects from their immediate environment. They begin understanding that objects have specific uses within their interactions with their parents (Rodríguez and Moro, 1999; Moro and Rodríguez, 2005). For example, when an infant takes a spoon and bangs it continuously on a plastic plate, a parent typically picks it up from the child and shows how it should be used. Progressively, infants come to master conventional uses, and therefore, they begin sharing a type of common ground with others. Empirical evidence suggests that 7-month-old infants do not use objects according to the conventions of their use but, rather, perform undifferentiated actions such as banging, throwing, and mouthing objects (Fenson et al., 1976). Several months later, by 14 months of age, infants start appreciating the socio-cultural use of objects when they manipulate objects (Rodríguez and Moro, 1999; Moro and Rodríguez, 2005).

Previous studies suggest that sharing conceptual common ground (i.e., how to use objects in conventional ways) transforms - both quantitatively and qualitatively - the nature of the communicative interaction between infants and parents, allowing for important developmental progression. For example, Dimitrova and Moro (2013) have shown that when infants and parents share common ground on the conventional use of objects, parents increase both the amount and the complexity of their gestures. Specifically, when 8-month-old infants did not show any mastery of the conventional use of the toys with which they played during triadic parent-infant-object interactions, parents produced clear-cut gestures repeatedly while exaggerating their movement. Later on, by 14 months of age, when children showed that they were progressively learning the conventional use of toys, parents started producing more complex gestures with brief and fast movement, without repeating them.

Evidence suggests that when infants begin sharing common ground on the communicative referents with their parent, communication becomes functionally meaningful. In other 
words, when the infant and the parent share a type of common knowledge, they become progressively more skilled in producing and understanding each other's communicative acts. Accordingly, in the theoretical framework that we provide, we argue that studying the communicative functions of infants' eye gaze should include a consideration of the level of common ground shared between the parent and the infant (second premise).

\section{Role of Parental Interpretations}

In the study of the functions of young children's first communicative gestures, Bates et al. (1975) showed a developmental progression between perlocutionary and locutionary acts. Namely, before young children's ability to convey a clear communicative function (illocutionary acts), the study of the function of infants' early communicative acts relies on the effect they trigger in the parent (perlocutionary acts). When parents are sensitive to an infant's communicative signals, they interpret them accurately and respond to them appropriately. Importantly, parental interpretations and responses help young children discover the contingencies between their communicative productions and the effect they elicit on the parent, thus laying the foundations of the development of communication and language (Bornstein et al., 1999; Tamis-LeMonda et al., 2001).

There is empirical evidence suggesting that parents interpret infants' early gestures based on shared knowledge about referents (Dimitrova et al., 2015). Studying the developmental progression of object use and of gesture production in 8- to 16-month-old infants, the authors found that when - by 14 months of age infants started to show a mastery of the conventional use of objects, parents began to interpret the communicative function of their infants' gestures referring to these objects as conveying a clear communicative function. The authors thus concluded that the level of common ground about the object shared between the parent and the infant was associated to the ability of parents to meaningfully interpret the function of their infant's gestures.

Given the intrinsic difficulty of accessing the communicative intention of infants' earliest communicative acts, analysis of the communicative function of infants' eye gaze should consider the interpretations provided by parents (third premise).

\section{DISCUSSION}

In this conceptual paper, we provide a theoretical framework for the study of the communicative function of infants' eye gaze. This theoretical framework relies on the following intertwined premises:

1. The centrality of the object: Beyond parent-child interactions in the first months of life when eye gaze primarily serves to establish connectedness and to share emotional states, studying the communicative function of infants' eye gaze when infants become able to integrate an external object into their interaction with the parent requires a consideration of the object.
2. The importance of common ground on object use: Stemming from the first premise, examining the communicative function of infants' eye gaze should take into consideration the level of common knowledge shared between the infant and the parent regarding the socio-cultural conventions of use of the referent of early communication, namely, the object.

3. The role of parental interpretations: When infants are at the early stages of their ability to convey communicative intentions, analysis of the function of infants' eye gaze should consider the parental interpretations of the function of the infant's gaze.

Based on previous studies on the importance of common ground on objects used for early communication (Dimitrova and Moro, 2013; Dimitrova et al., 2015), we argue that when infants and parents start sharing common ground on the conventional use of the objects/toys, parents become more likely to provide accurate interpretations of the function of their infants' eye gaze behavior. As described in early development, children's early communicative acts serve two primary functions: requesting something from the parent (i.e., proto-imperative) and sharing attention and interest with the parent (i.e., proto-declarative; see Bates et al., 1975). In order to study what young children mean with their eye gaze - and more specifically, how parents interpret the communicative function of their infants' eye gaze we argue that it is crucial to examine the knowledge parents and children share about the objects that they refer to in their communicative interactions. Importantly, the variability in children's mastery of the conventional use of objects is likely related to the variability in communicative functions that parents attribute to their children's eye gaze. Namely, when children are in the process of acquiring the conventional use of objects (i.e., proto-conventional use), the uncertainty and difficulty that they display in their object use might cue parents to interpret children's eye gaze, either as a request for help or assistance (i.e., proto-imperative function) or as an invitation to share attention and interest (i.e., proto-declarative function). However, when children master the conventional use of objects, the level of shared common ground should allow parents to interpret children's eye gaze as a way to establish joint attention and/or to seek validation or encouragement.

The theoretical premises provided in this paper are a first step toward the study of the functional aspects of eye gaze in early childhood. In order to examine the scientific soundness of this theoretical framework, empirical data are required. We recommend that analysis of the function of infants' eye gaze rely on naturalistic observations of parent-infant interactions with objects, where both the infants' level of mastery of the socio-cultural uses of objects (i.e., common ground) and the nature of parental responses to infant eye gaze are assessed. It is important to highlight that early intentional communication succeeds generally when different communicative means are solicited. Despite the fact that we focus on eye gaze, analysis of the functional aspects of infants' communication should include the multimodal means - such as vocalizations, gestures, and 
body postures - that infants mobilize together with eye gaze when communicating.

To our knowledge, this conceptual analysis is the first effort to provide a theoretical framework for the study of the functional aspects of eye gaze in early childhood. Indeed, despite the fact that eye gaze is a crucial component of interaction and communication (Kendon, 1967, 1990; Kleinke, 1986; Csibra and Gergely, 2006; Rossano, 2013), the communicative functions of child eye gaze are understudied. While pragmatic accounts of children's early communicative development are widespread in the literature, questions related to the underlying processes driving the development of children's abilities to convey different communicative functions remain understudied. Admittedly, there are various processes contributing to children's ability to communicate their intentions. Nonetheless, relying on the importance of the shared common knowledge about the main focus of early parent-child communication - namely, the object allows for a framework for understanding how infants develop their abilities to communicate intentionally.

In this conceptual analysis, we hypothesize that common ground shared between the parent and the infant about the use of an object would allow parents to interpret the function of their infants' eye gaze as communicatively meaningful. We argue that the knowledge on the communicative referent shared between the infant and the parent shapes the course of communicative behavior, constitutes and reflects the

\section{REFERENCES}

Adamson, L. B. (1996). Communication Development During Infancy. Boulder, CO: Westview Press.

Adamson, L. B., and Dimitrova, N. (2014). "Joint Attention and Language Development," in Encyclopedia of Language Development, eds P. Brooks and V. Kempe (SAGE), 299-304.

Argyle, M., and Cook, M. (1976). Gaze and Mutual Gaze. Cambridge, England: Cambridge University Press.

Austin, J. L. (1962). How to Do Things with Words. Oxford: O.U.P.

Bakeman, R., and Adamson, L. B. (1984). Coordinating Attention to People and Objects in Mother-Infant and Peer-Infant Interaction. Child Dev. 55, 12781289. doi: $10.2307 / 1129997$

Bates, E. (1976). Language and Context: The Acquisition of Pragmatics. New York: Academic Press.

Bates, E. (1979). "The emergence of symbols: Ontogeny and phylogeny," in Children's Language and Communication, Vol. 12, ed. W. A. Collins (New Jersey: Erlbaum), 121-156.

Bates, E., Benigni, L., Bretherton, I., Camaioni, L., and Volterra, V. (1979). The Emergence of Symbols: Cognition and Communication in Infancy. New York: Academic Press.

Bates, E., Camaioni, L., and Volterra, V. (1975). Performatives prior to speech. Merrill Palmer Q. 21, 205-226.

Bornstein, M. H., Tamis-LeMonda, C. S., and Haynes, O. M. (1999). First words in the second year: continuity, stability, and models of concurrent and predictive correspondence in vocabulary and verbal responsiveness across age and context. Infant Behav. Dev. 22, 65-85. doi: 10.1016/S0163-6383(99)80 006-X

Brazelton, T. B., Koslowski, B., and Main, M. (1974). "The origins of reciprocity: The early mother-infant interaction," in The Effect of the Infant on Its Caregiver, eds M. Lewis and L. A. Rosenblum (Oxford: Wiley-Interscience).

Brooks, R., and Meltzoff, A. N. (2014). "Gaze following: A mechanism for building social connections between infants and adults," in Mechanisms of Social Connection. From Brain to Group, eds M. Mikulincer and P. R. Shaver interactive function of gaze, and cues parents into tailoring their communicative response according to the infant's developmental needs, which in turns feeds back into the infant's social and communicative development.

\section{ETHICS STATEMENT}

Ethical review and approval were not required for the study on human participants in accordance with the local legislation and institutional requirements. The patients/participants (and their guardians in the case of participants under the age of 16) provided their written informed consent to participate in this study.

\section{AUTHOR CONTRIBUTIONS}

ND conceptualized the theoretical framework and wrote the manuscript.

\section{ACKNOWLEDGMENTS}

I would like to thank Prof. Christiane Moro from the University of Lausanne, Switzerland, for providing the infrastructure for this study.

(Washington, DC: American Psychological Association), 167-183. doi: 10. 1037/14250-010

Bruner, J. S. (1973). Organization of early skilled action. Child Dev. 44, 1-11. doi: $10.2307 / 1127671$

Bruner, J. S. (1974). From communication to language-A psychological perspective. Cognition 3, 255-287. doi: 10.1016/0010-0277(74)90012-2

Bruner, J. S. (1983). Child's talk: Learning to Use Language. New York, NY: Norton. Butterworth, G. E., and Jarrett, N. (1991). What minds have in common is space: Spatial mechanisms serving joint visual attention in infancy. Br. J. Dev. Psychol. 9, 55-72. doi: 10.1111/j.2044-835X.1991.tb00862.x

Campos, J. J. (1983). The importance of affective communication in social referencing. Merrill Palmer Q. 29, 83-87.

Casillas, M., and Hilbrink, E. (2020). "Communicative act development," in Developmental and Clinical Pragmatics, eds K. P. Schneider and E. Ifantidou (Germany: De Gruyter Mouton).

Clark, H. H. (1996). Uses of Language. Cambridge: Cambridge University Press.

Csibra, G. (2010). Recognizing communicative intentions in infancy. Mind Lang. 25, 141-168. doi: 10.1111/j.1468-0017.2009.01384.x

Csibra, G., and Gergely, G. (2006). "Social learning and social cognition: the case for pedagogy," in Processes of Change in Brain and Cognitive Development. Attention and Performance, eds Y. Munakata and M. H. Johnson (Oxford: Oxford University Press), 249-274.

Deák, G. O., Walden, T. A., Kaiser, M. Y., and Lewis, A. (2008). Drive from distraction: How infants respond to parents' attempts to elicit and re-direct their attention. Infant Behav. Dev. 31, 34-50. doi: 10.1016/j.infbeh.2007.06.004

Dimitrova, N. (2010). Culture in infancy. An account of a way the object "sculpts" early development. Psychol. Soc. 3, 77-91.

Dimitrova, N. (2013). It takes more than mean-end differentiation to intentionally communicate in infancy. A semiotic perspective on early communication development. J. Cult. Historic. Psychol. 3, 81-89.

Dimitrova, N. (2014). "L'usage de l’objet. Un lieu privilégié de l'émergence de la communication intentionnelle chez l'enfant," in Psychologie du Développement, Sémiotique et Culture, eds C. Moro and N. Muller-Mirza (France: Presses Universitaires du Septentrion), 79-98. 
Dimitrova, N., and Moro, C. (2013). Common ground on object use associates with caregiver's Gesturese. Infant Behav. Dev. 36, 618-626. doi: 10.1016/j.infbeh. 2013.06.006

Dimitrova, N., Moro, C., and Mohr, C. (2015). Caregivers interpret infants' early gestures based on shared knowledge about referents. Infant Behav. Dev. 3, 98-106. doi: 10.1016/j.infbeh.2015. 02.015

Fenson, L., Kagan, J., Kearsley, R., and Zelazo, P. (1976). The developmental progression of manipulative play in the first two years. Child Dev. 47, 232-36. doi: $10.2307 / 1128304$

Flom, R., Lee, K., and Muir, D. (2007). Gaze-Following. Its Development and Significance. Mahwah, NJ: Erlbaum.

Franco, F., and Butterworth, G. (1996). Pointing and social awareness: declaring and requesting in the second year. J. Child Lang. 23, 307-336. doi: 10.1017/ S0305000900008813

Ganea, P. A., and Saylor, M. M. (2007). Infants' use of shared linguistic information to clarify ambiguous requests for objects. Child Dev. 78, 493-502. doi: 10.1111/ j.1467-8624.2007.01011.x

Gibson, J. J. (1979). The Ecological Approach to Visual Perception. Boston, MA: Houghton Mifflin.

Golinkoff, R. M., Mervis, C. B., and Hirsh-Pasek, K. (1994). Early object labels: the case for a developmental lexical principles framework [*]. J. Child Lang. 21, 125-155. doi: 10.1017/S0305000900008692

Harding, C. G., and Golinkoff, R. M. (1979). The origins of intentional vocalizations in prelinguistic infants. Child Dev. 50, 33-40. doi: 10.2307/ 1129038

Kaye, K., and Fogel, A. (1980). The temporal structure of face-to-face communication between mother, and infants. Dev. Psychol. 16, 454-464. doi: 10.1037/0012-1649.16.5.454

Kendon, A. (1967). Some functions of gaze-direction in social interaction. Acta Psychol. 26, 22-63. doi: 10.1016/0001-6918(67)90 005-4

Kendon, A. (1990). Conducting Interaction. Patterns of Behavior in Focused Encounters. Cambridge: Cambridge University Press.

Kleinke, C. L. (1986). Gaze and eye contact: a research review. Psychol. Bull. 100, 78-100. doi: 10.1037/0033-2909.100.1.78

Klinnert, M., Campos, J., Sorce, J., Emde, R. N., and Sveyda, M. J. (1983). "Social referencing: An important appraisal process in human infancy," in The Emotions, Vol. 2, eds R. Plutchik and H. Kellerman (New York, NY: Academic Press).

Leung, E. H., and Rheingold, H. L. (1981). Development of pointing as a social gesture. Dev. Psychol. 17, 215-220. doi: 10.1037/0012-1649.17.2.215

Lewis, M. (1969). Infants' responses to facial stimuli during the first year of life. Dev. Psychol. 1, 75-86. doi: 10.1037/h0026995

Liebal, K., Behne, T., Carpenter, M., and Tomasello, M. (2009). Infants use shared experience to interpret pointing gestures. Dev. Sci. 12, 264-271. doi: 10.1111/j. 1467-7687.2008.00758.x

Meins, E. (1999). Sensitivity, security and internal working models: Bridging the transmission gap. Attach. Hum. Dev. 1, 325-342. doi: 10.1080/ 14616739900134181

Moll, H., Richter, N., Carpenter, M., and Tomasello, M. (2008). Fourteen-montholds know what 'we' have shared in a special way. Infancy 13, 90-101. doi: $10.1080 / 15250000701779402$

Moro, C., and Rodríguez, C. (2005). L'objet et la Construction de Son Usage chez le bébé. Une Approche Sémiotique du Développement Préverbal. Bern: Peter Lang.

Murphy, C. M., and Messer, D. J. (1977). "Mothers, infants and pointing: a study of a gesture," in Studies on mother-infant interaction, ed. H. R. Schaffer (New York, NY: Academic Press), 325-354.

Newson, J., and Newson, E. (1975). Intersubjectivity and the transmission of culture. Bull. Br. Psychol. Soc. 28, 437-445.

Patterson, M. L. (1983). Nonverbal Behavior: A Functional Perspective. New York, NY: Springer.

Rochat, P. (2001). The Infant's World. Cambridge, MA: Harvard University Press.
Rodríguez, C., and Moro, C. (1999). El uso convencional tambien hace permanentes a los objetos. Infancia Aprendizaje 84, 67-83. doi: 10.1174/ 021037098760378793

Rossano, F. (2013). "Gaze in interaction," in The Handbook of Conversation Analysis, eds J. Sidnell and T. Stivers (Malden: Wiley Blackwell), 308-329.

Scaife, M., and Bruner, J. S. (1975). The capacity for joint visual attention in the infant. Nature 253, 265-266. doi: 10.1038/253265a0

Schiffer, S. (1972). Meaning. New York, NY: Oxford University Press.

Searle, J. R. (1969). Speech Acts: An Essay in the Philosophy of Language. London: C.U.P.

Searle, J. R. (1975). "Indirect speech," in Syntax and Semantics, Speech Acts, eds P. Cole and J. L. Morgan (New York, NY: Seminar Press), 59-82.

Searle, J. R., and Vanderveken, D. (1985). Foundations of Illocutionary Logic. Cambridge: Cambridge University.

Stalnaker, R. C. (1978). “Assertion," in Syntax and Semantics: Pragmatics, ed. P. Cole (New York, NY: Academic Press), 315-332.

Stem, D. (1974). The goal and structure of mother-infant play. J. Am. Acad. Child Psychiatry 13, 402-421. doi: 10.1016/S0002-7138(09)61348-0

Stukenbrock, A. (2018). "Forward-looking: where do we go with multimodal projections?", in Modalities and Temporalities: Convergences and Divergences of Bodily Resources in Interaction, eds A. Deppermann and J. Streeck (Amsterdam: Benjamins), 31-68. doi: 10.1075/pbns.293.01stu

Stukenbrock, A., and Dao, A. N. (2019). "Joint Attention in Passing: What Dual Mobile Eye Tracking Reveals About Gaze in Coordinating Embodied Activities at a Market," in Embodied Activities in Face-to-face and Mediated Settings, eds E. Reber and C. Gerhardt (Cham: Palgrave Macmillan), 177-213. doi: 10.1007/ 978-3-319-97325-8 6

Tamis-LeMonda, C. S., Bornstein, M. H., and Baumwell, L. (2001). Maternal responsiveness and children's achievement of language milestones. Child Dev. 72, 748-767. doi: 10.1111/1467-8624.00313

Tomasello, M. (1992). First Verbs: A Case Study of Early Grammatical Development. Cambridge, MA: Cambridge University Press.

Tomasello, M. (2003). Constructing a Language: A Usage-Based Theory of Language Acquisition. Cambridge, MA: Harvard University Press.

Tomasello, M. (2008). Origins of Human Communication. Cambridge, MA: MIT Press.

Trevarthen, C., and Hubley, P. (1978). "Secondary intersubjectivity: Confidence, confiding and acts of meaning in the first year," in Action, gesture, and symbol: The emergence of language, ed. A. Lock (New York, NY: Academic Press), 183-229.

Tronick, E., Als, H., and Adamson, L. (1979). "Structure of early face to face communicative interactions," in Before Speech: The Beginning of Interpersonal Communication, ed. M. Bullowa (Cambridge: Cambridge University Press).

Volkmar, F., and Mayes, L. (1990). Gaze behavior in autism. Dev. Psychopathol. 2, 61-69. doi: 10.1017/S0954579400000596

Volkmar, F. R. (1987). “Social Development," in Handbook of Autism, eds D. Cohen and A. Donnelan (New York: Wiley).

Werner, H., and Kaplan, B. (1963). Symbol Formation: An OrganismicDevelopmental Approach to the Psychology of Language. New York, NY: Wiley.

Wittgenstein, L. (1962). "The blue book," in Philosophy in the Twentieth Century, Vol. 2, eds W. Barrett and D. H. Aiken (New York, NY: Random House).

Conflict of Interest: The author declares that the research was conducted in the absence of any commercial or financial relationships that could be construed as a potential conflict of interest.

Copyright (C) 2020 Dimitrova. This is an open-access article distributed under the terms of the Creative Commons Attribution License (CC BY). The use, distribution or reproduction in other forums is permitted, provided the original author(s) and the copyright owner(s) are credited and that the original publication in this journal is cited, in accordance with accepted academic practice. No use, distribution or reproduction is permitted which does not comply with these terms. 


\section{OPEN ACCESS}

Edited by:

Federico Manzi,

Catholic University of the Sacred

Heart, Italy

Reviewed by:

Elisabetta Lombardi,

Catholic University of the Sacred

Heart, Italy

Cattaruzza Elisa,

Université de Neuchâtel, Switzerland

*Correspondence:

Michela Ponticorvo

michela.ponticorvo@unina.it

Specialty section

This article was submitted to

Developmental Psychology,

a section of the journal

Frontiers in Psychology

Received: 20 December 2019

Accepted: 27 March 2020

Published: 08 May 2020

Citation:

Ponticorvo M, Sica LS, Rega A and Miglino O (2020) On the Edge

Between Digital and Physical:

Materials to Enhance Creativity

in Children. An Application to Atypical Development. Front. Psychol. 11:755.

doi: 10.3389/fpsyg.2020.00755

\section{On the Edge Between Digital and Physical: Materials to Enhance Creativity in Children. An Application to Atypical Development}

\author{
Michela Ponticorvo ${ }^{1 *}$, Luigia Simona Sica ${ }^{1}$, Angelo Rega ${ }^{1,2}$ and Orazio Miglino ${ }^{1,3}$ \\ 'Natural and Artificial Cognition Laboratory, Department of Humanistic Studies, University of Naples "Federico II", Naples, \\ Italy, ${ }^{2}$ Institute for Research, Training and Information on Disabilities (IRFID), Neapolisanit, Ottaviano, Italy, ${ }^{3}$ Institute \\ of Cognitive Sciences and Technologies, National Research Council, Rome, Italy
}

The 4 P's creativity model (person, process, press, and product) underlines how creativity is strongly connected with the materials employed to conceive and realize a creative outcome. As a multiform construct, it invites a wide variety of approaches to the study of it. One of the most promising ways to address this issue is to connect it with cognitive development and related educational pathways, as creativity can be enhanced and stimulated in every child, leading to an improvement both at personal and societal level. Even if creativity is recognized and highly valued, there is still a lack of methods which can stimulate creativity in an effective way. Useful hints may come from the outstanding contributions of Piaget and Montessori who underlined that interaction with the physical world is a fundamental building block for cognitive development. In this paper, starting from these fixed points, we describe some creativity enhancing methods for children which give importance to the edge between digital and physical materials. Digital materials open new ways to the use and integration of physical materials with hybrid platforms which can be used in educational contexts. Together with this perspective we provide a description of the application of these methodologies to enhance creativity in children with Autistic Spectrum Disorder.

Keywords: educational materials, creativity, game-based learning, video-modeling, Autis Spectrum Disorder

\section{INTRODUCTION: WHERE CREATIVITY IS}

A general definition of creativity describes it as the ability to generate ideas, insights and solutions that are original, flexible (Amabile, 1996; Sternberg and Lubart, 1996) and effective (Runco and Jaeger, 2012). A vast body of research has been conducted in this field from different points of view (psychological, pedagogical, educational, etc.). In brief, creativity can be understood as the combination of several factors (Treffinger et al., 1983; Houtz and Krug, 1995a,b) of both a cognitive (primarily related to divergent thinking) and an emotional type (primarily related to creative personality). On the cognitive side, there is a general convergence on the notion that creative outputs result from cognitive flexibility (flexible and divergent ways of thinking) and cognitive persistence (persistent and systematic way of thinking) (see Dietrich and Kanso, 2010). On the 
emotional side, Williams (1994) explored the emotionaldivergent aspect of creativity, identifying the main characteristics of personality as: (1) curiosity (the capacity to investigate elements and ideas, finding new and not always direct and obvious connections); (2) complexity (the tendency to look for new alternatives and solutions to problems, to restore order out of chaos); (3) imagination (the ability to visualize mental images); (4) risk-taking (the inclination to act under unstructured conditions and to defend one's own ideas).

Creativity can be also seen as the result of interaction between the individual, the domain, and the field. For instance, Rhodes (1961, 1987) developed the 4 P's model (Figure 1), which places creativity at the interplay of four distinct strands, i.e., process (the different stages of a creative activity), person (the characteristics of individuals), press (the qualities of the environment where creativity happens), and product (the tangible or intangible outcomes of the creative process). Rhodes' classification has become a major framework for the holistic exploration of creativity.

However, creativity is not only the production of something new for the entire society (like arts): creativity is often found in an individual's everyday activities. In this sense, literature defines two types of creativity: Creativity and creativity. "Capital C" creativity brings into existence something genuinely new that receives social validation (enhances culture) and is related to an accomplishment that consists of a clear-cut, eminent creative contribution. "Small C" creativity refers to ideas or products that are new to the person, but only to the person; it is therefore more focused on everyday activities, such as those creative actions in which everyone can be involved every day (Figure 1). Kaufman and Beghetto (2009) add another 2 Cs to their model, including a new category, a "little-c" for the little-c category, mini-c (Beghetto and Kaufman, 2007), which is connected with the learning process. Mini-c is defined as the novel and personally meaningful interpretation of experiences, actions, and events (Beghetto and Kaufman, 2007). Together with Mini-c they introduce Pro-c, the developmental and effortful progression beyond little-c, not reaching Big- $\mathrm{C}$ status, on professional-level expertise in creative areas. The 5 A's framework (Glãveanu, 2013) goes beyond the 4 P's

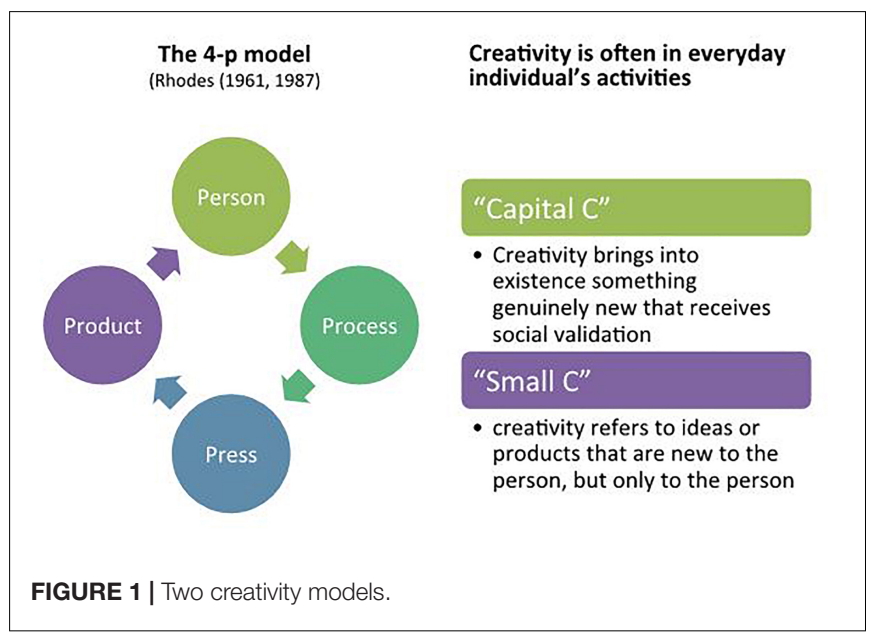

model to give a new perspective on creativity: it introduces actor, action, artifact, audience, and affordances.

Considering these contributions, creativity is a precious resource for the positive psychological development of all individuals (with normative and non-normative developmental trajectories). In these terms, mainly considering the four P's model and the Small $\mathrm{C}$ description, as shown in Figure 1, we deal with it.

In the present paper we aim at delineating some methods that can be applied to stimulate creativity in children with typical and atypical developmental trajectories, employing both digital and physical materials and joining the notable advantages that these kinds of materials can offer. After a description of the connection between creativity and interaction with physical materials, we describe an application of this method to a concrete case of atypical development. In particular we report in section "Fostering emotion recognition to stimulate creativity by technology in children with Autistic Spectrum Disorder (ASD)" an example of how to stimulate creativity by promoting emotion recognition with digital and physical materials in children with Autistic Spectrum Disorder (ASD).

\section{Creativity From a Developmental Point of View}

From what we said, it is clear that creativity can also be seen as a cognitive resource along the "life-span," starting from childhood. Indeed, children tend to exhibit a natural disposition toward creativity and expression; fluency (the ability to generate multiple ideas) and flexibility develop across adolescence with distinct trajectories for divergent thinking and insight (Kleibeuker et al., 2013), explorative thinking characterizes middle adolescence (Johnson and Wilbrecht, 2011). Moreover children can be sensitive to creativity outcomes from 3 years of age, which is quite early (Di Dio et al., 2007), and this sensitivity can enable them to develop their creative side. In children with atypical developmental trajectories, creativity can offer support for adaptive processes (Hetzroni et al., 2019). If not stimulated, creativity can diminish and follow a negative bending. For instance, creativity diminishes by $40 \%$ between the ages of five and seven. This is due to the beginning of formal schooling with its educational rules which may inhibit commitment to creative thinking and behaviors (Amabile, 1996; McCormick and Plugge, 1997). New research has also led to a new understanding of the capacity for positive change and creative expression in the second half of life (Cohen, 2006). In general terms, psychological literature has highlighted that creativity can be interpreted as an individual resource, as a potent predictor of social problemsolving and adjustment (Ogoemeka, 2011). In other words, creative individuals are remarkable for their ability to adapt to almost any situation and exploit whatever possible to reach their goals (Csikszentmihalyi, 1996).

The paths to support individual development do not always consider creativity as a useful resource for well-being, despite research providing evidence to this effect. The role of creativity as a resource for individual well-being was identified: creativity and learning are strictly connected not only during childhood 
but also during young adulthood and adulthood (see Gajda et al., 2017); long-term participation in creative activities has benefits for middle-aged and older people as they may improve their adaptation to later life (Adams-Price et al., 2018).

Even if we consider childhood, which is probably the most studied phase of development for supporting creativity in learning contexts, there is still a lack of methods for stimulating creativity in an effective way. In this paper we describe some methods for enhancing creativity in children that give importance to the fertile edge between digital and physical materials.

\section{Creativity: How to Stimulate It}

The crucial added element in Rhode's vision is the "press" or the environment. This dimension focuses on the characteristics of the environment (social, cultural, and political; Simonton, 1999) as crucial factors for supporting/hindering creativity. In addition, Csikszentmihalyi (1996) highlighted some environmental features which may foster creativity, including training, expectations, resources, recognition, and some factors which may hinder creativity, like time pressure, evaluation, lack of respect, and competition.

For Harris and de Bruin (2018) creativity is "an essential aspect of teaching and learning that is influencing worldwide educational policy and teacher practice, shaping the possibilities

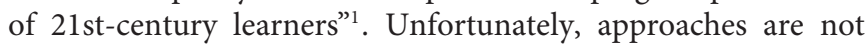
always coordinated with each other and often have characteristics of extemporaneousness and occasionality. As recent work shows, there is a strong will to help teachers in enhancing creativity without the need for special programs or training, encouraging it during teachers' regular work (Beghetto, 2013) or their work associated with the common core (Beghetto et al., 2014; Giglio and Cruz-Ortiz, 2015). In this sense, Karwowski's et al. (2015) words on the relationship between creativity and education are enlightening:

"Since the beginning of creativity theory, the educational side of creativity has been at the heart of scholars' thinking and research (p. 165). However, to help teachers stimulate creativity effectively, a better understanding of mechanisms underlying creativity is necessary (p.166)".

In the field of education and pedagogy, creativity can be defined as "purposive imaginative activity generating outcomes that are original and valuable in relation to the learner" (Cremin et al., 2013; see also Craft et al., 2014).

We believe also that a thorough reflection on the tools used in the educational dialogue to stimulate the creative process is even more necessary because it ties together relational processes, cognitive processes and the instrument's own characteristics (in terms of potential and risks). Physical objects, digital tools, and "materials" in general thus become our specific object of investigation. Moreover, thanks to the massive entrance of technological devices in all aspects of our everyday life, it is important that the concept of creativity is rethought considering these elements.

${ }^{1}$ https://oxfordre.com/education/view/10.1093/acrefore/9780190264093.001. 0001/acrefore-9780190264093-e-383

\section{CREATIVITY AND INTERACTION WITH THE PHYSICAL WORLD DURING INDIVIDUAL PSYCHO-SOCIAL DEVELOPMENT}

In order to better understand how technology can be connected to creativity in a developmental perspective, it is useful to go back to the contribution of relevant authors in the field who underline that the interaction with the physical world is fundamental in structuring cognitive processes.

Children explore the world around them by relying on their sensorimotor functions: in their infancy (from a few months to when they enter kindergarten) the hands are the main channel for acquiring knowledge. Children point, handle, touch, taste, smell, and manipulate while understanding an object's features and functions.

As time goes by, adults do not spend the same amount of time in pointing, reaching, touching and manipulating, but the manipulative acts in the physical world become internal processes, cognitive functions and neural structures: an action is no more run in the physical world, but is simulated in the virtual space represented by the mind (Smith and Gasser, 2005).

Even if these functions become virtual, the use of hands and of the body as a whole remains fundamental: the physical world is mirrored on the cognitive side and the dynamic interaction at the edge of physical and cognitive is a resource for various cognitive processes, including creativity.

Piaget (1952, 1964), whose theorization analyzed in detail how children develop their cognition, recognizes a fundamental role for the interaction with the physical universe in shaping development at a cognitive level. In his well-known definitions of assimilation and adaptation, what comes from the external world is important also in terms of physical interaction. In this perspective, cognitive processes emerge with doing and interacting.

Vygotsky (1978), on the other hand, underlined the role of context in shaping learning and development, because, in his opinion, cognitive development is the result of an interaction between the child and the social context he/she is immersed in. The cognitive functions of a growing child are built from what happens in their social interactions (consider for example language and thought). Even if for this author the physical world is shaded, it is anyway out of doubt that social interactions happen and are mediated by physical interactions, especially during childhood.

Bruner (1961), in his theory, took some elements from Piaget, i.e., the learner active involvement and from Vygotsky, i.e., the importance of social context. Then he formulated an approach to learning cognitive development which is defined as cultural. Bruner's learning theory states that it is a complex activity where we can recognize three underlying interacting processes: (1) acquisition of information, (2) transformation of information in a new form that allows problem solving and (3) checking the efficacy of this transformation.

Bruner gives central importance to culture and to the active participation of children for their cognitive development. Bruner 
in fact stated that "the active participation in the learning process by the child might result in the following hypothesized benefits: an increase in intellectual potency so as to make the acquired information more readily viable in problem solving, the action of the learning activities in terms of the intrinsic reward of discovery itself (as contrasted with the drive-reduction model of learning), learning the heuristics of discovery, and making material more readily accessible in memory" (Bruner, 1961, p. 21).

Starting from Bruner, other authors in the cognitive field have stressed this issue; in their contribution Papert (1980) and Jonassen (1994) underline the active role of the people who get to understand their experience by exploiting cultural tools in a context. In this approach, which has been named in constructionism, "meaningful learning" (Jonassen et al., 2008) and "discovery learning" (Papert and Harel, 1991) are important conceptual contributions and cannot be neglected when reflecting on creativity and technology.

More recently, the perspective of embodied and situated cognition (Clark, 1998; Shapiro, 2019) puts the concept of action at the center together with physical interaction. In embodied cognition theory, the organism with its sensory and motor apparatus interacts with the external environment and structures its cognitive processes through this interaction. This means that the body, by means of its continuous exchange with the world, both physical and social, determines how cognition develops. The motor and the perceptual apparatus allow the constant flow of information between the internal and external side that sediment in the cognitive processes.

For the situated learning theories (Clancey, 1995) the theoretical core is that the subject (who knows) cannot be considered as different and separated from the object (what is known). Moreover this process does not happen in a vacuum, but in a context defined by social constraints (Rambusch, 2006). Along with this approach, it is important to underline that the body is a relevant medium of exchange between person and context. This argument dates back to Piaget.

For Piaget and his seminal theory, the body is the first instrument, in terms of time and of importance, to get to know the world. Indeed in his theory, the sensorimotor stage is the first phase in which children both with typical and atypical development exploit his/her body to explore the environment. More recently the contribution by Galperin $(1969,1989)$ supports the idea that body is fundamental for mind: the mental objectoriented activity derives from the object-oriented activity which is run in the physical world, at the beginning. In brief, the physical manipulation of tangible objects is the starting point of what is later internalized and becomes human thought. Galperin's approach has been considered by the later contribution of Rambusch and Ziemke (2005) as a bridge, a connection between situated learning and embodied cognition theories.

Using Rambush and Ziemke words, we can say that "the embodied cognition is in many aspects a very social process, and that embodied social process such as mimicry and imitation are significant for social relations as they help people connect, making it possible for them to communicate and to understand each other." The work of Roth (2002) falls perfectly in this trace. In fact he showed that gestures are not only a reflection of what has been learnt but also contribute to new acquisition, because they have the function to communicate to the external world together with helping to make things clearer and more understandable for the speaker itself.

In the field of education science, relevant approaches have been affirmed along the years which hold, at their core, the importance of child-active involvement. Let us consider the Montessori (2013) approach, which suggests using methodologies where children are at the center, acting with special materials that stimulate child senses, for example, smelling jars, the broad stair and the pink tower. Children play with these materials in a way that promote their independence in learning and their problem-solving ability, together with peer cooperation. This leads to an active education methodology that can be fruitfully applied in the acquisition of cognitive and social skills, including creativity.

In this approach, the hands play a fundamental role. In Montessori's (1995) words, hands are instruments of intelligence, they become an extension of thought and can become a vehicle to stimulate creativity, confirming what has been said about Embodied Cognition. In our opinion, the described contributions underlying the importance of the body in exploring and acquiring knowledge about the word have important implications in the field of education and in the development of creativity. As underlined by Stanciu (2015), the core ideas of embodied cognition can have a notable effect on the discussion on creativity in cognitive science, especially in the domain of everyday creativity, the little-c, but also with the forms which result in culturally relevant, highly regarded products and artifacts (e.g., Kaufman and Beghetto, 2009).

We therefore believe that creativity can be stimulated keeping in mind the contribution from Embodied Cognition theory, allowing to exploit the process in which the body and the environment can shape creativity. Creativity in the Embodied Cognition framework can help us to understand the impact that physical and body activities have on creative thinking, also on typical and atypical development. This means that techniques based on Embodied Cognition can foster a creative output.

In the next section we will introduce some tools and methods used to enhance creativity in children, based on the described theoretical framework. Moreover we will introduce, in section "Fostering emotion recognition to stimulate creativity by technology in children with Autistic Spectrum Disorder (ASD)", a possible application of the tools and methods used to stimulate creativity in children with Autistic Spectrum Disorder as reported in section "Methods to enhance creativity in children: playing implies learning in a creative way".

\section{METHODS TO ENHANCE CREATIVITY IN CHILDREN: PLAYING IMPLIES LEARNING IN A CREATIVE WAY}

"We can identify creative processes in children at the very earliest ages, especially in their play. A child who sits astride a stick and pretends to be riding a horse; a little girl who plays with a doll and imagines she is its mother; a boy who in his games becomes 
a pirate, a soldier, or a sailor, all these children at play represent examples of the most authentic, truest creativity. (...) A child's play is not simply a reproduction of what he has experienced, but a creative reworking of the impressions he has acquired" (Vygotsky, 1967; Engl. Transl., 2004, p. 11). We can start our discourse from this relationship between creativity and play identified by Vygotsky. That is also our argument: stimulating play can stimulate learning, and both imply creative processes. More specifically, to learn by exploring reality (objects, rules and roles, questions, problems) is a process involving curiosity, flexibility, divergent thinking, and risk-taking, all of which are creative processes.

During the entire developmental arc, from birth to elder age, playing a game stimulates the player to start a challenge, either cognitive (card games, board games, role games) or physical (competition in sport, dancing, fine manipulative activities). This challenge leads the player to learn something (or to exercise already acquired abilities), and so playing a game can be a good approach to transferring some knowledge or skill at every age (Dell'Aquila et al., 2016).

What we have said about games in general is also valid for digital games. These have become very widespread nowadays and are often used in a game-based learning approach. Indeed Tobias et al. (2014) in their fundamental review on this issue have shown that using video and computer games is an effective way to enhance the cognitive processes underlying learning. This effectiveness is strengthened if game design and instructional design are integrated to exploit at the maximum level the motivating features of games. In fact, games are exceptionally good at involving people and increasing their motivation to solve problems, promoting learning (Kapp, 2012).

Along with game-based learning, technology-enhanced learning (TEL), technology-enabled learning or technologyenhanced education (TEE) are a wide ensemble of educational methodologies based on digital technologies that give importance to interactivity in the learning process, active experience and collaborative knowledge building (Goodyear and Retalis, 2010).

TEL includes Serious games, Augmented Reality, Educational Robotics and Multiplayer Virtual Worlds, educational technologies, e-learning, technology-enhanced classrooms, etc. TEL can be used to design and implement different kinds of technology-supported learning that is strongly activity-based. In fact TEL has some relevant features that can be fruitfully employed to stimulate cognitive processes: interactivity, collaboration, communication, personalization, etc.

TEL allows children to have more control of their own cognitive processes, to build up knowledge, and become part of the teaching process, both individually and as a group. Indeed collaboration between peers becomes relevant and can be very well supported in a TEL context. This means that TEL can lead to really engaging learning experiences where different tools can be used to build personalized learning pathways. As children today are more and more exposed to and attracted by technologies, TEL can exploit this attractive power.

The introduction of TEL can effectively exploit students' potential in a tailored scenario, and motivate children through creativity and customized resources. On the educator side, this means that they modify their pedagogy and educational models to make education an active process.

Game-based learning (with digital games) and technologyenhanced learning are two methodological approaches that strongly rely on digital elements, but they do not neglect physical materials. In particular there are some technologies that allow the bridging of these sides (Figure 2).

In this paper we support the use of technological tools in education, but we should be aware that there is also the danger of an excessive or distorted use of technology in educational processes (Desmurget, 2019). In our opinion children should use technology accompanied by someone (an adult or a peer), which promotes and frames the process of knowledge building with technology (Ponticorvo et al., 2018b).

In the next subsection we will introduce some of the processes that can be employed to integrate physical and digital materials.

\section{Bridging Physical and Digital Materials: TUI and Smart Objects}

In recent years, the progress made in the technological field has enabled the possibility of more interfaces. Interfaces allow the joining of two different entities, two separate domains. In the field of information science and what is interesting for the present paper, some interfaces are particularly relevant: the TUI, tangible user interfaces (Blackwell et al., 2007; Ishii, 2008). In this case what is joined is the edge between the digital and physical dimensions so as that a user can interact with information (or data) at the digital level by interacting with the physical environment-for example manipulating tangible objects at a cognitive level (which is very relevant), as we have seen in the previous section.

If we consider the education field, allowing the connection between physical and digital dimensions offers new opportunities under the approach of TEL. It is possible, in fact, to integrate tangible and physical materials traditionally used in education into a new life in the digital universe. This way educational materials such as logic blocks, cards, counting chips, rods, manipulatives in general, etc. (Di Fuccio et al., 2015, 2016; Ferrara et al., 2016; Ponticorvo et al., 2018a,b, 2019) become Smart Objects (Kortuem et al., 2009). A Smart Object can have sensors and processors that, thanks to software, can be in

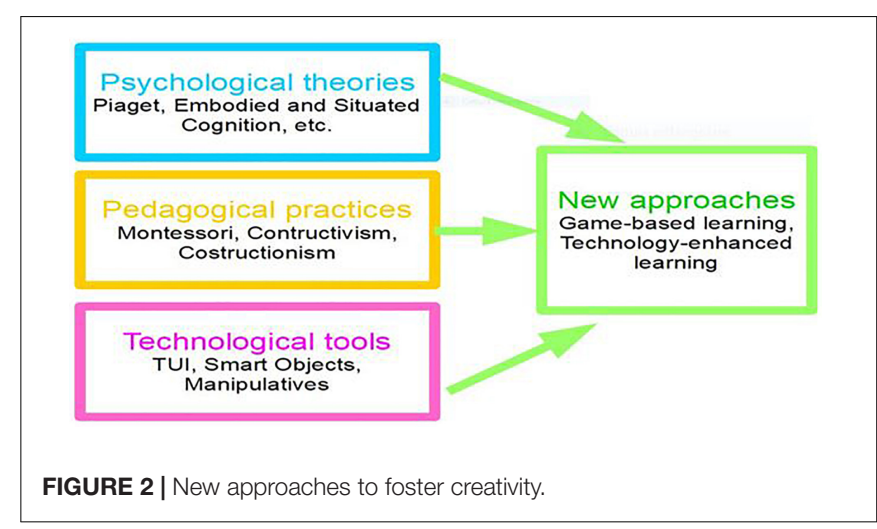


connection and process information together with other objects and with the user.

In sum, Smart Objects are computationally enhanced versions of everyday objects that can also process data, exploit sensors to get information and affect their environment; moreover they can be connected one with the other thanks to the Internet, generating an Internet of Things (IoT). IoT ( $\mathrm{Li}$ et al., 2015) and Smart Objects technologies have been introduced in the educational domain, offering opportunities and challenges (Domínguez and Ochoa, 2017). Indeed the technological tools can widen the space and time devoted to education, can overcome barriers and open new educational pathways, but this introduction must follow an educational framework that prevents the drawbacks and threats of technology in education (Selwyn, 2016; Collins and Halverson, 2018). Using Tangible User Interfaces (TUI) allows the physical embodiment of digital information, extending the accessibility of objects in the physical world (including tangible materials and active surfaces) which allows the joining together of the digital world and physical objects (see Figure 2).

TUI, Smart objects, and manipulatives represent the technological tools and instruments through which it is possible to put into practice the described approaches of game-based learning and technology-enhanced learning, based on the pedagogical approaches that value the active construction of knowledge and the psychological theories described above that give importance to active interactions with the physical world for framing cognition. This is represented in Figure 2, where it is possible to find a sketch of the connections between these three elements.

In the next section we will describe the application of these principles in a case-study where technology is used to stimulate creativity through its emotional side in a non-verbal participant with autism.

\section{FOSTERING EMOTION RECOGNITION TO STIMULATE CREATIVITY BY TECHNOLOGY IN CHILDREN WITH AUTISTIC SPECTRUM DISORDER (ASD)}

As hinted at in the introduction, creativity is connected with the emotional sphere. Emotional competence (Saarni, 1999) refers to the ability to deal effectively with emotions, in terms of understanding others' emotions, expressing appropriate emotions in a certain context, and regulating them in order to adapt to specific situations. Positive emotional states can increase creativity (Fredrickson et al., 2003), leading to the production of more ideas, even if these ideas aren't necessarily more original. Also, negative emotions like sadness, anger, and disappointment can help the individual to generate more ideas.

Creativity and emotions are strongly connected in children that have a typical development, as shown by different studies. Fluidity, an aspect that describes the production of a good number of ideas, and flexibility, producing original ideas, appear to have a significant link to emotional competence (Sánchez-Ruiz et al., 2011; Hoffmann and Russ, 2012). If we consider a nonnormative population, such as children with Autism Spectrum Disorder, there are some studies that focus on creativity and show that these children can have a high degree of creativity, together with some difficulties related to emotional competence.

Recent studies have shown that for people with ASD video-modeling is particularly effective. Video-modeling is a methodology, integrated with technology, whose functioning is based on learning by observation and imitation. This means that children exposed to video-modeling, starting from the digital material represented by the video, can reproduce what they see with their own bodies, acting on the physical side.

Video-modeling, in more detail, consists in the observation of a video, in which a model shows a target behavior, and the subsequent imitation of that model. A lot of individuals with autism benefit from visually cued instructions (Frith, 2003). Moreover, studies indicated that video-modeling is effective for learning emotional skills: this methodology can support children with autism to recognize emotions and to perceive and respond appropriately to facial expressions (Axe and Evans, 2012; Chen et al., 2016).

Individuals with ASD often present difficulties in the expressive behavior of their emotions, especially in modulating the expression of their face based on the affective state experienced. The expression of emotions is sometimes absent, sometimes ambiguous; in addition, individuals with ASD experience major difficulties in recognizing and interpreting emotional expressions in others. Difficulties in emotion expression and recognition can lead to frustration and dysfunctional behaviors. Furthermore, the poor understanding of one's emotional state can influence the creative capacities of a child.

\section{Method}

In this paper we describe a methodological approach to fostering creativity acting on the emotional side, by video-modeling. The goal was helping a child with ASD to learn and develop emotional skills, particularly the communication of their own and others' emotional states, and to verify if the acquisition of emotion recognition was useful for fostering creativity in a drawing task. The chosen methodological design is a multiple baseline across behaviors, A-B-A type, commonly used in ABA treatments. This research design A-B-A belongs to the single-case study where with letter $\mathrm{A}$ we intend the baseline condition, whereas the letter $\mathrm{B}$ indicates the treatment condition. With an example: $A$ is a situation of anxiety; B is the treatment for reducing anxiety. We identify a real effect of the treatment when the curve undergoes a significant change. The treatment phase starts when the behavior is stable. The target behavior is measured during both phases and results are then compared. Some variants of AB experimental design can foresee a stricter control on variables to have stronger conclusions. With ABA (also called reversal design), in this context, we refer to a research design built on $\mathrm{AB}$ that then integrates a follow-up phase after the treatment that includes repeated measures (Horner et al., 2005). The participant was a 6-year-old child with a mild-level condition of Autism Spectrum Disorder. 
During the intervention, the following target behaviors were identified:

- To show correctly an emotional state through the change of face expressions; for emotion facial expression, reference was made to the theory by Ekman and Friesen (2003), as it provided useful guidance in experiments on emotion.

- To properly label an emotional state, therefore recognizing and naming an own emotional state.

- To correctly label an emotional state experienced by others, therefore recognizing and naming the emotional state shown by others through facial expression and vocalization.

The categories of emotions selected for the intervention were joy, anger and surprise: these emotions can vary in intensity but are universally recognized and expressed in the same way.

The baseline sessions allowed the tracing of the baseline for the child, whereas the intervention consisted of a videomodeling session.

Data were collected using the traditional procedure of the Momentary Time Sample Recording (MTSR) (Powell et al., 1975) that is to correctly show the emotional state of joy through the change of the face. MTSR is a data-recording technique that is usually used when the observed behavior is not easily quantifiable.

\section{Results}

Results indicated that video-modeling was effective for the acquisition of social and emotional behavior skills in children with ASD, and this led to an improvement of creativity as shown in drawing. The task given to the child consists of recognizing the appropriate emotion and drawing the expression of the mouth to fit the picture, considering all the elements in the face (eyes, eyebrows, nose and signs on cheeks).

In fact, as it is possible to observe in Figure 3, compared with the children's drawing before and after the video-modeling intervention, children were able to recognize the emotions and to reproduce them in a more appropriate and richer way in their productive drawing.

Figure 3 shows the ability to recognize emotions by the child with ASD is ameliorated between BEFORE (on the left) and AFTER (on the right) the treatment with video-modeling. We can observe that discrimination of emotion is better on the right, where the drawn mouth is more coherent with the face elements.

The present study represents a first example of the application of the core ideas of this paper to stimulate creative processes in children with atypical developmental trajectories. It has many limitations that will be overcome with the definition of a research methodology to be applied to a wider group of participants, and also to children with typical development.

\section{DISCUSSION AND CONCLUSION}

Creativity has both a cognitive and emotional side and it is strongly connected with the materials that are employed

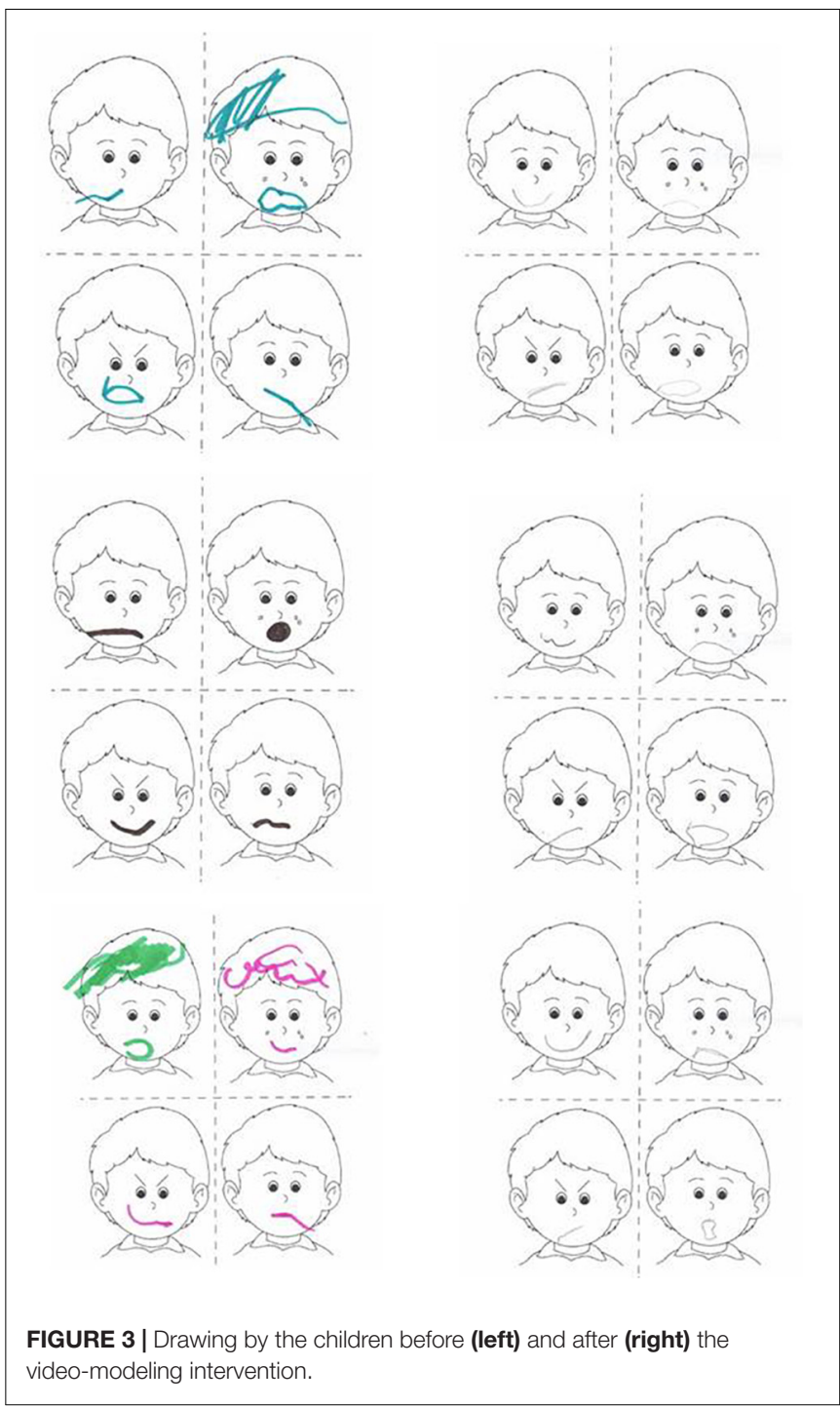

during creative processes. In this paper we have tried to put together these dimensions to propose methodologies and tools that can be used to stimulate creativity in children. In particular we have shown that educational materials that involve both digital and physical materials, at the edge between these domains, can be particularly effective in having positive effects on creativity mediated by cognitive functions and emotional processes: this happens through the body and the interactions it has with the physical world.

Moreover, we have introduced some useful indications deriving from intervention in a non-normative sample, that is children with ASD. It is also possible to observe in this case that creativity can be improved by stimulating emotional competence and this can be done using materials that bridge digital and physical borders. The reported study is only the starting point of a validation pathway that will cover research on children with typical development, adults and elder people. In fact, these results will be further challenged by widening the sample 
involved in the experiment, proposing more interventions with different materials and trying this methodology on other groups in different phases of development. In particular, as elderly people can benefit from creativity stimulating intervention, the next step will involve aged people with and without impairments. The experimental design will also be enriched so as to study these wider samples longitudinally and transversally, and experimental protocols will be introduced to have a stronger control over variables.

Creativity can be a resource for everyone in every phase of life: stimulating it with physical and digital materials can be effective. The present study showed an example of how to follow this route, which will be further investigated and developed.

\section{DATA AVAILABILITY STATEMENT}

The datasets generated for this study are available on request to the corresponding author.

\section{ETHICS STATEMENT}

Ethical review and approval was not required for the study on human participants in accordance with the local legislation and institutional requirements. Written informed consent to

\section{REFERENCES}

Adams-Price, C. E., Nadorff, D. K., Morse, L. W., Davis, K. T., and Stearns, M. A. (2018). The creative benefits scale: connecting generativity to life satisfaction. Int. J. Aging Hum. Dev. 86, 242-265. doi: 10.1177/009141501769 9939

Amabile, T. M. (1996). Creativity and Innovation in Organizations. Cambridge, MA: Harvard Business School.

Axe, J. B., and Evans, C. J. (2012). Using video modeling to teach children with PDD-NOS to respond to facial expressions. Res. Autism Spectr. Disord. 6, 1176-1185.

Beghetto, R. A. (2013). Killing Ideas Softly: The Promise and Perils of Creativity in the Classroom. Charlotte: IAP Information Age Publishing.

Beghetto, R. A., and Kaufman, J. C. (2007). Toward a broader conception of creativity: a case for "mini-c" creativity. Psychol. Aesthet. Creat. Arts 1, 73-79.

Beghetto, R. A., Kaufman, J. C., and Baer, J. (2014). Teaching for Creativity in the Common Core Classroom. New York, NY: Teachers College Press.

Blackwell, A. F., Fitzmaurice, G., Holmquist, L. E., Ishii, H., and Ullmer, B. (2007). "Tangible user interfaces in context and theory," in CHI'07 Extended Abstracts on Human Factors in Computing Systems, eds M. B. Rossom and D. Gilmore (New York, NY: ACM Association for Computing Machinery), 2817-2820.

Bruner, J. S. (1961). The Act of Discovery, Vol. 31. Cambridge, MA: Harvard Educational Review, 21-32.

Chen, C. H., Lee, I. J., and Lin, L. Y. (2016). Augmented reality-based videomodeling storybook of nonverbal facial cues for children with autism spectrum disorder to improve their perceptions and judgments of facial expressions and emotions. Comput. Hum. Behav. 55, 477-485.

Clancey, W. J. (1995). “A tutorial on situated learning," in Proceedings of the International Conference on Computer and Education, ed. J. Self (Charlottesville, VA: AACE), 49-70.

Clark, A. (1998). Being there: Putting Brain, Body, and World Together Again. Cambridge, MA: MIT press. participate in this study was provided by the participants' legal guardian/next of kin.

\section{AUTHOR CONTRIBUTIONS}

MP, LS, and OM conceived the original plan of the manuscript. AR designed and carried out the experiment. MP took the lead in writing the manuscript with the support of LS and AR. MP, LS, $\mathrm{OM}$, and AR discussed the manuscript draft and contributed to the submitted manuscript.

\section{FUNDING}

This research has been supported by the EU projects Digital Creativity Enhanced in Teacher Education (DoCENT) (20171-IT02-KA203- 036807) under Erasmus + programme and SemAntically integrating Genomics with Electronic health records for Cancer CARE (SAGE-CARE) funded by Horizon 2020 Research and Innovation programme under the Marie Skaodowska-Curie (grant agreement no. 644186).

\section{ACKNOWLEDGMENTS}

We thank the children, their parents and therapists involved in the experiments for their participation.

Cohen, G. (2006). Research on creativity and aging: the positive impact of the arts on health and illness. Generations 30, 7-15.

Collins, A., and Halverson, R. (2018). Rethinking Education in the Age of Technology: The Digital Revolution and Schooling in America. New York, NY: Teachers College Press.

Craft, A., Cremin, T., Hay, P., and Clack, J. (2014). Creative primary schools: developing and maintaining pedagogy for creativity. Ethnogr. Educ. 9, 16-34.

Cremin, T., Chappell, K., and Craft, A. (2013). Reciprocity between narrative, questioning and imagination in the early and primary years: examining the role of narrative in possibility thinking. Think. Skills Creat. 9, 135-151. doi: 10.1016/j.tsc.2012.11.003

Csikszentmihalyi, M. (1996). Creativity: The Work and Lives of 91 Eminent People. New York, NY: Harper Collins.

Dell'Aquila, E., Marocco, D., Ponticorvo, M., Di Ferdinando, A., Schembri, M., and Miglino, O. (2016). Educational Games for Soft-Skills Training in Digital Environments: New Perspectives. Cham: Springer International Publishing Switzerland.

Desmurget, M. (2019). La Fabrique du Crétin Digital-Les Dangers des Écrans Pour nos Enfants. Paris: Le Seuil.

Di Dio, C., Macaluso, E., and Rizzolatti, G. (2007). The golden beauty: brain response to classical and renaissance sculptures. PLoS One 2:e1201. doi: 10. 1371/journal.pone.0001201

Di Fuccio, R., Ponticorvo, M., Di Ferdinando, A., and Miglino, O. (2015). “Towards hyper activity books for children. connecting activity books and montessorilike educational materials," in Design for Teaching and Learning in a Networked World, eds G. Conole, T. Klobučar, C. Rensing, and J. E. Lavoué (Cham: Springer), 401-406.

Di Fuccio, R., Ponticorvo, M., Ferrara, F., and Miglino, O. (2016). "Digital and multisensory storytelling: narration with smell, taste and touch," in European Conference on Technology Enhanced Learning, eds K. Verbert, M. Sharples, and T. Klobucar (Cham: Springer), 509-512. 
Dietrich, A., and Kanso, R. (2010). A review of EEG, ERP, and neuroimaging studies of creativity and insight. Psychol. Bull. 136, 822-848. doi: 10.1037/a0019749

Domínguez, F., and Ochoa, X. (2017). "Smart objects in education: an early survey to assess opportunities and challenges," in Proceedings of the 2017 Fourth International Conference on eDemocracy \& eGovernment (ICEDEG), (Quito: IEEE), 216-220.

Ekman, P., and Friesen, W. V. (2003). Unmasking the Face: A Guide to Recognizing Emotions from Facial Clues. Los Altos, CA: ISHK Institute for the Study of Human Knowledge.

Ferrara, F., Ponticorvo, M., Di Ferdinando, A., and Miglino, O. (2016). "Tangible interfaces for cognitive assessment and training in children: LogicART", in Smart Education and e-Learning 2016, eds V. Uskov, R. J. Howlett, and C. Jain Lakhmi (Cham: Springer), 329-338.

Fredrickson, B. L., Tugade, M. M., Waugh, C. E., and Larkin, G. R. (2003). What good are positive emotions in crisis? A prospective study of resilience and emotions following the terrorist attacks on the United States on September 11th, 2001. J. Pers. Soc. Psychol. 84, 365-376. doi: 10.1037//0022-3514.84.2.365

Frith, U. (2003). Autism: Explaining the Enigma. Hoboken NJ: Blackwell Publishing.

Gajda, A., Karwowski, M., and Beghetto, R. A. (2017). Creativity and academic achievement: a meta-analysis. J. Educ. Psychol. 109, 269-299.

Galperin, P. I. (1969). "Stages in the development of mental acts," in A Handbook of Contemporary Soviet Psychology, eds M. Cole and I. Maltzman (New York, NY: Basic Books), 249-273.

Galperin, P. I. (1989). Mental actions as a basis for the formation of thoughts and images. Sov. Psychol. 27, 45-64.

Giglio, M., and Cruz-Ortiz, J. (2015). Creative Collaboration in Teaching. New York, NY: Palgrave Macmillan.

Glãveanu, V. P. (2013). Rewriting the language of creativity: the five A's framework. Rev. Gen. Psychol. 17, 69-81.

Goodyear, P., and Retalis, S. (2010). Technology-Enhanced Learning. Rotterdam: Sense Publishers.

Harris, A., and de Bruin, L. R. (2018). Secondary school creativity, teacher practice and STEAM education: an international study. J. Educ. Change 19, 153-179.

Hetzroni, O., Agada, H., and Leikin, M. (2019). Creativity in autism: an examination of general and mathematical creative thinking among children with autism spectrum disorder and children with typical development. J. Autism Dev. Disord. 49, 3833-3844. doi: 10.1007/s10803-019-04094-х

Hoffmann, J., and Russ, S. (2012). Pretend play, creativity, and emotion regulation in children. Psychol. Aesthet. Creat. Arts 6, 175-184.

Horner, R. H., Carr, E. G., Halle, J., McGee, G., Odom, S., and Wolery, M. (2005). The use of single-subject research to identify evidence-based practice in special education. Except. Child. 71, 165-179. doi: 10.1044/0161-1461(2009/08-0128)

Houtz, J. C., and Krug, D. (1995a). Assessment of creativity: resolving a mid-life crisis. Educ. Psychol. Rev. 7, 269-300.

Houtz, J. C., and Krug, D. (1995b). Preface to special issue on the educational psychology of creativity: toward an educational psychology of creativity? Educ. Psychol. Rev. 7, 137-139.

Ishii, H. (2008). The tangible user interface and its evolution. Commun. ACM 51, 32-36.

Johnson, C., and Wilbrecht, L. (2011). Juvenile mice show greater flexibility in multiple choice reversal learning than adults. Dev. Cogn. Neurosci. 1, 540-551. doi: $10.1016 /$ j.dcn.2011.05.008

Jonassen, D. H. (1994). Technology as cognitive tools: learners as designers. ITForum Pap. 1, 67-80.

Jonassen, D. H., Howland, J., Marra, R., and Crismond, D. (2008). Meaningful Learning with Technology. Upper Saddle River, NJ: Pearson.

Kapp, K. M. (2012). The Gamification of Learning and Instruction. San Francisco, CA: Wiley

Karwowski, M., Gralewski, J., and Szumski, G. (2015). Teachers' effect on students' creative self-beliefs is moderated by students' gender. Learn. Individ. Differ. $44,1-8$.

Kaufman, J. C., and Beghetto, R. A. (2009). Beyond big and little: the four c model of creativity. Rev. Gen. Psychol. 13, 1-12.

Kleibeuker, S. W., De Dreu, C. K., and Crone, E. A. (2013). The development of creative cognition across adolescence: distinct trajectories for insight and divergent thinking. Dev. Sci. 16, 2-12. doi: 10.1111/j.1467-7687.2012.01176.x
Kortuem, G., Kawsar, F., Sundramoorthy, V., and Fitton, D. (2009). Smart objects as building blocks for the internet of things. IEEE Internet Comput. 14, $44-51$.

Li, S., Da Xu, L., and Zhao, S. (2015). The internet of things: a survey. Inf. Syst. Front. 17, 243-259.

McCormick, D. J., and Plugge, C. D. (1997). "If I am an artist, what's wrong with my picture? Rediscovering your creativity in a grown-up world," in Proceedings of the Annual AEE International Conference Deeply Rooted, Branching'Out, 1972-1997, Asheville.

Montessori, M. (1995). The Absorbent Mind. New York, NY: Henry Holt and Company Inc.

Montessori, M. (2013). The Montessori Method. Piscataway, NJ: Transaction publishers.

Ogoemeka, O. H. (2011). Emotional intelligence and creativity in teacher education. Int. J. Soc. Sci. Educ. 3, 124-129.

Papert, S. (1980). Mindstorms: Children, Computers, and Powerful Ideas. New York, NY: Basic Books.

Papert, S., and Harel, I. (1991). Situating constructionism. Constructionism 36, 1-11. doi: $10.1177 / 1049732313488837$

Piaget, J. (1952). The Origins of Intelligence in Children. New York, NY: International Universities Press.

Piaget, J. (1964). Part I: cognitive development in children: Piaget development and learning. J. Res. Sci. Teach. 2, 176-186.

Ponticorvo, M., Dell'Aquila, E., Marocco, D., and Miglino, O. (2019). Situated psychological agents: a methodology for educational games. Appl. Sci. 9:4887.

Ponticorvo, M., Di Fuccio, R., Ferrara, F., Rega, A., and Miglino, O. (2018a). "Multisensory educational materials: five senses to learn," in Proceedings of the International Conference in Methodologies and Intelligent Systems for Technology Enhanced Learning, eds T. Di Mascio, P. Vittorini, R. Gennari, F. De la Prieta, S. Rodríguez, M. Temperini, et al. (Cham: Springer), 45-52.

Ponticorvo, M., Rega, A., and Miglino, O. (2018b). "Toward tutoring systems inspired by applied behavioral analysis," in Proceedings of the International Conference on Intelligent Tutoring Systems, eds R. Nkambou, R. Azevedo, and J. Vassileva (Cham: Springer), 160-169.

Powell, J., Martindale, A., and Kulp, S. (1975). An evaluation of time-sample measured of behaviour 1. J. Appl. Behav. Anal. 8, 463-469.

Rambusch, J. (2006). "Situated learning and Galperin's notion of object-oriented activity," in Proceedings of the Annual Meeting of the Cognitive Science Society, Skövde.

Rambusch, J., and Ziemke, T. (2005). "The role of embodiment in situated learning," in Proceedings of the 27th Annual Conference of the Cognitive Science Society, (Mahwah, NJ: Lawrence Erlbaum), 1803-1808.

Rhodes, M. (1961). An analysis of creativity. Phi Delta Kappa 42, 305-310.

Rhodes, M. (1987). "An analysis of creativity," in Frontiers of Creativity Research: Beyond the Basics, ed. S. Isaksen (Buffalo, NY: Bearly Ltd), 216-222.

Roth, W. M. (2002). From action to discourse: the bridging function of gestures. Cogn. Syst. Res. 3, 535-554.

Runco, M. A., and Jaeger, G. J. (2012). The standard definition of creativity. Creat. Res. J. 24, 92-96.

Saarni, C. (1999). The Development of Emotional Competence. New York, NY: Guilford Press.

Sánchez-Ruiz, M. J., Hernández-Torrano, D., Pérez-González, J. C., Batey, M., and Petrides, K. V. (2011). The relationship between trait emotional intelligence and creativity across subject domains. Motiv. Emot. 35, 461-473.

Selwyn, N. (2016). Education and Technology: Key Issues and Debates. London: Bloomsbury Publishing.

Shapiro, L. (2019). Embodied Cognition. Abingdon: Routledge.

Simonton, D. K. (1999). Origins of Genius: Darwinian Perspectives on Creativity. Oxford: Oxford University-Press.

Smith, L., and Gasser, M. (2005). The development of embodied cognition: six lessons from babies. Artif. Life 11, 13-29. doi: 10.1162/1064546053278973

Stanciu, M. M. (2015). Embodied creativity: a critical analysis of an underdeveloped. Procedia Soc. Behav. Sci. 187, 312-317.

Sternberg, R. J., and Lubart, T. I. (1996). Investing in creativity. Am. Psychol. 51, 677-688.

Tobias, S., Fletcher, J. D., and Wind, A. P. (2014). "Game-based learning," in Handbook of Research on Educational Communications and Technology, eds 
J. M. Spector, M. D. Merrill, J. Elen, and M. J. Bishop (New York, NY: Springer), 485-503.

Treffinger, D. J., Isaksen, S. G., and Firestien, R. L. (1983). Theoretical perspectives on creative learning and its facilitation: an overview. J. Creat. Behav. 17, 9-17.

Vygotsky, L. (1978). Interaction between learning and development. Read. Dev. Child. 23, 34-41.

Vygotsky, L. S. (1967). Play and its role in the mental development of the child. Sov. Psychol. 5, 6-18.

Williams, F. (1994). TCD. Test Della Creatività e del Pensiero Divergente. Trento: Centro Studi Erickson.
Conflict of Interest: The authors declare that the research was conducted in the absence of any commercial or financial relationships that could be construed as a potential conflict of interest.

Copyright (๑) 2020 Ponticorvo, Sica, Rega and Miglino. This is an open-access article distributed under the terms of the Creative Commons Attribution License (CC BY). The use, distribution or reproduction in other forums is permitted, provided the original author(s) and the copyright owner(s) are credited and that the original publication in this journal is cited, in accordance with accepted academic practice. No use, distribution or reproduction is permitted which does not comply with these terms. 


\section{OPEN ACCESS}

Edited by:

Giulia Savarese,

University of Salerno, Italy

Reviewed by:

Luca Tateo,

University of Oslo, Norway

Kristiina Kumpulainen,

University of Helsinki, Finland

*Correspondence:

Giovanna Barzanò

dott.giovanna.barzano@gmail.com

Specialty section:

This article was submitted to Developmental Psychology,

a section of the journal

Frontiers in Psychology

Received: 13 January 2020 Accepted: 16 April 2020

Published: 03 June 2020

Citation:

Barzanò G, Amenduni F, Cutello G, Lissoni M, Pecorelli C, Quarta R, Raffio L, Regazzini C,

Zacchilli E and Ligorio MB (2020)

When the Place Matters: Moving the Classroom Into a Museum to Re-design a Public Space.

Front. Psychol. 11:943

doi: 10.3389/fpsyg.2020.00943

\section{When the Place Matters: Moving the Classroom Into a Museum to Re-design a Public Space}

\author{
Giovanna Barzanò ${ }^{*}$, Francesca Amenduni², Giancarlo Cutello ${ }^{3}$, Maria Lissoni ${ }^{4}$, \\ Cecilia Pecorelli5, Rossana Quarta ${ }^{3}$, Lorenzo Raffio ${ }^{6}$, Claudia Regazzini', \\ Elena Zacchilli ${ }^{8}$ and Maria Beatrice Ligorio $^{9}$
}

\begin{abstract}
${ }^{1}$ Department of Curriculum and Evaluation, Ministry of Education, University and Research, Rome, Italy, ${ }^{2}$ Department of Educational Sciences, Roma Tre University, Rome, Italy, ${ }^{3}$ Academy of Arts and New Technologies, Rome, Italy, ${ }^{4}$ Scuola Secondaria di I Grado Statale Norberto Bobbio, Turin, Italy, ${ }^{5}$ Azienda Speciale Palaexpo, Rome, Italy, ${ }^{6}$ Tony Blair Institute for Global Change, London, United Kingdom, ${ }^{7}$ Luigi Settembrini Comprehensive Institute, Rome, Italy, ${ }^{8}$ Niccolò Machiavelli State High School Florence, Rome, Italy, ${ }^{9}$ Department of Educational Sciences, Psychology, Communication, University of Bari Aldo Moro, Bari, Italy
\end{abstract}

In this case-report we describe an experience where alternative places - rather than the classroom - are exploited to implement learning processes. We maintain that this experience is a good example of materiality because it focuses on a project where students had the opportunity to re-design a public space. To this aim, various objects and tools are used to support discussions and exchanges with new stakeholders. Our theoretical vision combines Piaget's and Vygotsky's tradition with an innovative framework called the Trialogical Learning Approach (TLA). From such theoretical background an idea of materiality emerges, that refers to material in combination with the social relationships developed around the material. Our case-report concerns a participatory project run by Rete Dialogues, a national school network focusing on global citizenship education. Our research question is: how can this project highlight the connection between the TLA and socio-materiality? Since 2017, around 200 students (age 7-16) and 20 teachers from different schools have been engaged in tackling the degradation of an important square in Rome. The project - "Dialogues in the Square" (DiS) was developed with several stakeholders that contributed to the understanding of critical issues influencing the maintenance of the square, in the perspective of planning, and possibly implementing improvements proposed by students. Crucial is the cooperation with two important urban art projects: (i) the pilot-project MACRO-ASILO, run by the MACRO museum in Rome and aimed at connecting the world of art with the city life; (ii) the "building sites" of the Rome Rebirth Forum, inspired by the worldknown artist Michelangelo Pistoletto's "third paradise" methodology, that encourages responsibility and action taking on sustainability through art. Drawing on data collected through direct observations and video recordings, we aim to show and make sense of the connection between the TLA and socio-materiality, highlighting three key elements: the flexible use of mediation tools, the overcoming of the dichotomy between individual and collective learning through reflection, and the re-shaping of social practices.

Keywords: sociomateriality, urban space, global citizenship education, dialogue, sustainable development goals, Trialogical Learning Approach 


\section{INTRODUCTION}

The way students learn is still attracting theoretical and practical attention. New definitions of learning and teaching are sought. Academics and experts are now focusing their research on several dimensions previously neglected or misunderstood, such as creativity, collaboration, action competency, communication competency, and space-time relevance (Bellanca, 2010; Hallgarten et al., 2015; Kober, 2015; Nielsen, 2015; Ritella et al., 2016). Traditional learning does not appear to be able to target these dimensions; therefore, a fresh look at educational practices is needed. After having discussed the theoretical underpinnings of our approach, this paper describes a project where materiality is introduced as the empowering dimension that supports the transaction between different learning contexts. We focus on some aspects of the learning processes that have occurred in one of the sessions within our project. Our intent is to make sense of the impact of materiality from two complementary perspectives: the materiality of the learning object (a square in Rome, Piazza Annibaliano) and the materiality of the working environment (a particular room in a modern art museum in Rome, the "words room," set up for the MACRO-ASILO project).

\section{THEORETICAL BACKGROUND}

Where do children learn about the world? How do students form their own ideas? The literature offers a number of answers to these questions, determining both the theoretical vision of how cognition works and the ideal practical setting for effective learning processes. For decades, theories about these topics have assumed the form of a contraposition/polarity between a Piagetian-based and a Vygotskian-based approach.

According to Piaget, knowledge resides in objects, and children retrieve information by manipulating them (Piaget and Inhelder, 1967). It is by querying the elements composing the context in which children are immersed and by making hypotheses about how objects will react to actions performed "on" them that they gather information about the world (Spelke, 1991), whereas for Vygotskij (1978), the main source of learning is social interaction. It is by observing and imitating adults and, later, by engaging collaboratively in joint actions that children learn and make sense of the world around them. Objects are important, but they become sources of information through social interactions, first based on adult imitation and later by appropriating and internalizing the actions observed.

An attempt to reconcile these two approaches into a wider vision has been offered by frameworks such as situated learning (Anderson et al., 1996; Cobb and Bowers, 1999), Activity Theory (Engeström, 1999), socio-constructivism and cultural psychology (Bruner, 1996; Cole, 1998), and most recently, the Trialogical Learning Approach (TLA) (Paavola and Hakkarainen, 2014; Sansone et al., 2016).

All these approaches share the idea of learning as a complex process that interests the individual sphere, as well as group work, and is influenced by the context and the instruments/tools used. In particular, the TLA integrates three different perspectives: (i) a "monologic" vision of learning, focused on individual increments of knowledge; (ii) the dialogic viewpoint that stresses the relevance of dialogue and encounters of different perspectives; and (iii) the intentional processes involved in the production of collaborative artifacts, connoted by a real meaning and utility. This approach responds to the demands of training competences for the twenty-first century, such as the skill to work with knowledge and to contribute actively to the development of modern society (Karlgren et al., 2020). Furthermore, it capitalizes on insights coming from the socio-constructivism and the cultural approach by giving relevance to context and situated dynamics.

The TLA calls for the construction of the so-called trialogical objects. These objects are addressed to a community that is different from the one in which they are built. To have recipients from another community gives sense to confrontation, contamination of practices, and ways of thinking. Therefore, learners become professionals of knowledge building, capable of creating valuable material objects containing knowledge, which can then be exploited outside school or academic contexts. When objects are used in concrete situations, they create further knowledge through processes of confrontation, generation of ideas, and creativity. Learning becomes a strategy to solve emerging problems and to constantly seek new and innovative ideas. Environments intentionally designed for knowledge innovation, equipped with technological tools, are needed to transform students' intangible ideas into digital entities (Hakkarainen, 2009).

Within the traditional TLA framework, materiality is still underdeveloped. The focus on building objects that embody conceptual knowledge and shared ideas and the relevance of tools as instruments that foster cognitive and social processes and support the construction of objects are hints of an implicit materiality or rather socio-materiality. Illuminating is Latour's (2005), challenge (2005) when he asks the reader to define a soldier. Through this simple thought experiment, he concludes that there are no soldiers without their uniforms and arms. They co-constitute each other and determine their relationship by identifying the formation they belong to.

Sørensen (2009) uses the term materiality to refer to both the material and the social relationships developed around the material. This definition is particularly helpful when objects are digital. Johri (2011) proposes "socio-materiality as a key theoretical perspective that can be leveraged to advance research, design and use of learning technologies in the practice tradition" (p. 210). The use of technology makes it clear that learning is both inherently material and social or socio-material (Orlikowski and Scott, 2008). When talking about digital environments and tools, the inseparability of material and social elements is essential (Barad, 2003; Barzanò and Grimaldi, 2013).

The theoretical concept of materiality is operationalized in different pedagogically oriented strategies such as Object-Based Learning. For example, Mayorga (2019) reports that while handling museum Objects, primary school students start to think differently and to reinterpret the cultural artifacts. Mirza (2016) observes that the material dimension assumes the function of a medium through which primary school children project their 
own emotions or those of another person or convey information and contribute to knowledge construction.

Thus, materiality is not just a matter of adding a new dimension; it means highlighting the relevance of considering "things" as real partners of cognitive and social processes, as elements containing knowledge and supporting the generation of new knowledge. This knowledge is not simply acquired by touching, manipulating, or experimenting with "things"; rather, it is defined through social actions, cultural processes of sensemaking, and intersubjective construction of mutual exchange of values about the objects. Where and with what this is occurring matters because it contributes to shaping these processes.

\section{THE CASE STUDY}

The case study presented here aims at providing empirical evidence of the role of socio-materiality in shaping learning processes. We also highlight how the TLA helps to emphasize the socio-material dimension, crossing the boundaries between formal (classroom) and informal (museum, the square) learning spaces. This will allow us to answer our research question: how can this project highlight the connection between the TLA and socio-materiality?

The session we analyze has been developed within a project called Dialogues in the Square (DiS). Started in 2017 - and still active - it has involved over 200 students from primary school (age 7) to upper-secondary school (age 16) and 20 teachers, in two schools situated in central Rome: Istituto Comprensivo Settembrini and Liceo Machiavelli. Within a framework of activities targeting global citizenship education (Reimers, 2009; Sobe, 2012; Reimers et al., 2016) and sustainable development goals run by a national school network (retedialogues.it), students started brainstorming about their environment, focusing on the needs of a nearby well-known square in Rome: Piazza Annibaliano. This important space, recently restored (2014), was soon left in a dangerous abandonment. A new metro station, situated in a context of ancient monuments, is now surrounded by litter and unfinished flowerbeds, left uncultivated. Students were encouraged to observe the square and engage in planning its regeneration: their plans are conceived as trialogical objects, i.e., knowledge that they create addressing communities external to the school. Moreover, negotiations were started with the municipality to have their support, resulting in a formal memorandum of understanding (MOU) with the schools. Artists/experts in various fields were involved to help students figure out suitable actions to undertake to improve the state of the square, eliciting its potential as a social and artistic site.

In 2019, an important opportunity arose: a well-known museum of modern art - MACRO, not far from the schools and the square - launched its pilot project MACRO-ASILO aimed at promoting the connection between citizens and art and making its spaces available to artists or citizens with ideas to present. In particular, the MACRO's "words room" appeared to be the ideal venue to work on the DiS project. This is a classroomstyle room equipped with rolling chairs and tables and with an enormous traditional blackboard, measuring $22 \times 3 \mathrm{~m}$. The museum also became the venue of the Rome Rebirth Forum, an ongoing initiative promoted by the world-renowned artist Michelangelo Pistoletto to enact his idea of the "third paradise," involving artists and social actors to develop and spread a deeper awareness on sustainability issues. The DiS project became an active member of the forum and benefited from the opportunity to invite several artists to cooperate.

Several sessions took place in the "words room," where different classes worked with/on the blackboard to accomplish "planning activities" concerning Piazza Annibaliano. Students sketched their proposals after lively discussions with artists/experts. Each session was public, had a title, was scheduled ahead, and was published in the museum's catalog: invited guests and occasional visitors were welcome, allowing students to share and discuss their performance with various audiences (see a detailed visual presentation of the full project in the Supplementary Material).

In the next paragraph, we will describe the setting, the available equipment, and how tools became partners of students' cognitive and social processes.

\section{A New Learning Space: Getting Into the MACRO Museum "Words Room"}

In this section, we analyze one particular event taking place in the MACRO museum's "words room," focusing on the learning environment, the materials used, and their impact on participants' reactions and interactions undertaking the task. In this session, a single class is involved, composed of 27 pupils aged 12 (grade seven, 15 girls, 12 boys) from mixed socioeconomic backgrounds. They are familiar with the square, as they all live nearby. The class is very active within the DiS project; nevertheless, it is their first time in the MACRO museum. The session is observed and videotaped: our data consist of extracts from students' dialogues as well as "thick descriptions" (Denzin, 2001) elaborated by the external observer.

It is 7 February 2019, from 10.30 to 13.30 , when our class goes to the museum with their art and technology teachers to meet Rachid Benhadj, a leading Italo-Algerian film director particularly interested in diversity and intercultural dialogue (see Figure 1). The students know him, having watched one of his videos. As is the case for artists/experts in other sessions, he was invited to support students' creative process of elaborating the idea of the "square" as a venue for proposals and new atmospheres that can add value and expand the possibilities of inhabitants and visitors.

In a preliminary meeting in the museum hall, five teams (four or five students each) are formed, following the teachers' suggestions. Benhadj presents his proposal to the students: "Think deeply of Piazza Annibaliano, figure out new settings, and portray them following the wave of your dreams: how would you like the square and why, pushing your imagination as far as possible..." Students are, therefore, invited to elaborate the idea of the "square" representing their ideals, without worrying about feasibility at this stage. With this task in mind, they enter the "words room," and it is clear how impressed they are from the beginning by its lights, the arrangement of the rolling furniture, and the giant blackboard. A connection between thinking/doing

${ }^{1}$ http://www.pistoletto.it/eng/crono26.htm. 


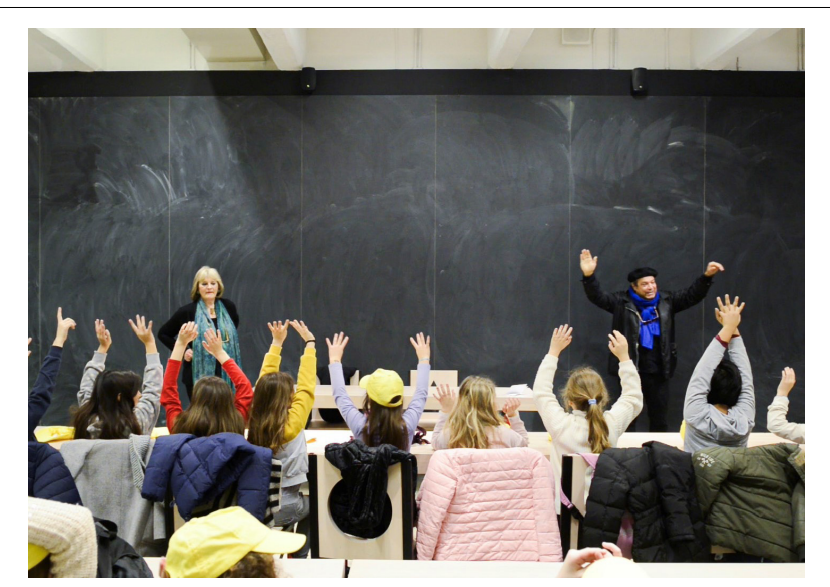

FIGURE 1 | Film director Rachid Benhadj introducing the exercise to the students. Images (C) Martina Pavia, graduating student at Academy of Arts and New Technologies, Rome, Italy.

is thus made evident, and students are encouraged to go back and forward from immateriality to materiality - as we will see in the next paragraph.

\section{At Work: Flexible Use of Mediation Tools}

Benhadj sketches a quick map of Piazza Annibaliano and surrounding streets at the center of the blackboard and better clarifies the expected delivery: paper-and-pencil sketches to start, and then the teams will move to the blackboard to represent their project with colored chalks.

Now that the task is clear, students start working on white sheets. Talking becomes intense, ideas are shared, and sketches circulate within/between teams. Technology comes into play naturally; no need for adults to suggest it. For instance, phones become cameras to store pictures that make possible comparisons and overviews crucial to inspire the work on the blackboard. Finally, about $45 \mathrm{~min}$ after starting, the five teams position themselves around the map sketched by Benhadj, easily defining their action space on the blackboard (see Figure 2).

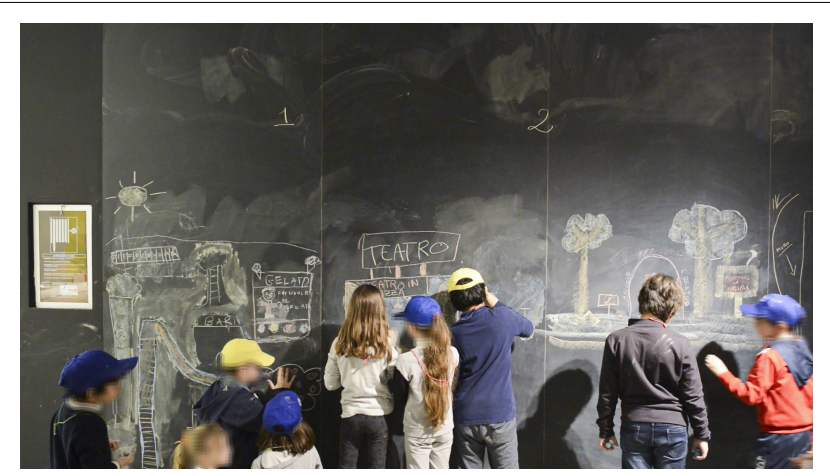

FIGURE 2 | Students sketch their ideas for the square. Images (C) Martina Pavia, graduating student at Academy of Arts and New Technologies, Rome, Italy.
The "genius loci" of the room lies in the alteration of the dimensions of traditional tools used in the classroom. This setting ends up disregarding a consolidated stereotype: the blackboard is by definition an "exclusive" place generating a markedly vertical relationship. It is used by a single person - or a few - who is expected to report something to an audience to whom the back is turned. Here the blackboard is "open to all": the teams work horizontally and simultaneously, observing one another's work and sharing ideas. Apparently, the confusion is remarkable, but the works develop efficiently; students' active engagement is visible. Someone moves his or her chair near the blackboard, others use the ladder available in the room, and someone else even sits on the shoulders of a friend to use the space at the top of the board. Others shoot videos or take pictures.

Even the colored chalks become important actors, with their immediate but fragile effectiveness enabling creativity (see Figure 3). Paradoxically, the awareness that whatever was created can disappear with just a few passes of the eraser pushes students to refine their work: "to take pictures before it disappears," as a student clarifies.

What has been described so far provides first evidence of how the TLA could enhance the socio-material dimension of learning. This approach emphasizes the flexible use of technologies and mediation tools. Depending on what students want to achieve - create, store, transform - they move from using their smartphones to using chalks, always as a tool to shape their ideas and to "materialize" them.

\section{Reflecting on the Work}

In about one hour, the blackboard is lively, full of shapes, colors, and writings, and the time comes for a collective report (see Figure 4). Benhadj poses two questions: "What have you done, can you tell us?" And then: "Were there emotions in this work? What touched you the most?" Each team gets ready for their presentation, while someone enjoys looking at their work from a distance, video-recording a full overview of the blackboard. The teams "walk" along the blackboard, stopping in front of each drawing to deliver the presentations: students naturally swing from the role of presenters to that of audience. Feedback is intense.

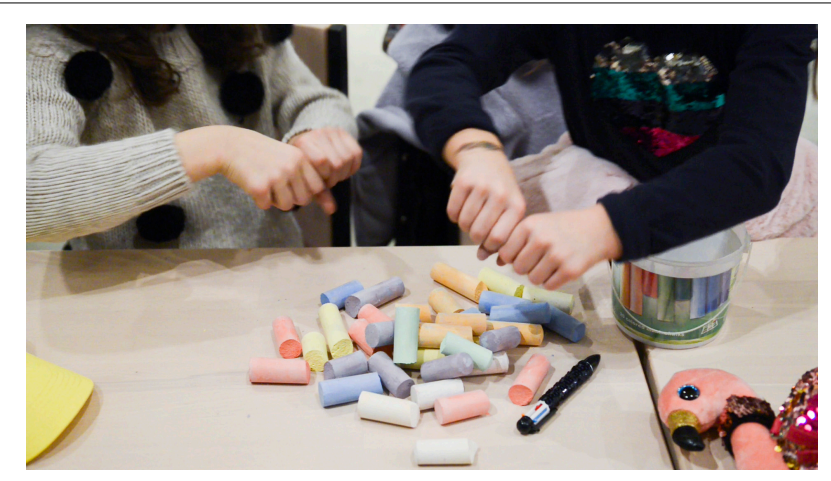

FIGURE 3 | Chalks acted as an enabler of creativity. Images (C) Martina Pavia, graduating student at Academy of Arts and New Technologies, Rome, Italy. 


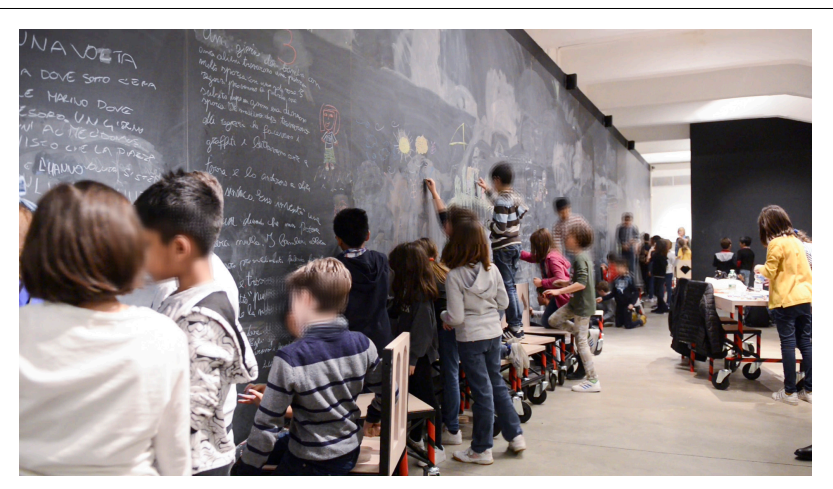

FIGURE 4 | Students using the full length and height of the blackboard. Images (C) Martina Pavia, graduating student at Academy of Arts and New Technologies, Rome, Italy.

Proposals are detailed, rich in inventions and strategies. They include: architectural and decorative elements, green spaces, and many solutions about how to make them work. Director Benhadj is very pleased; he listens carefully and interacts with students, to their great satisfaction. For example, project 3 presents a wall specifically created to welcome graffiti artists. Next to the sketch, some guidelines appear on how to organize periodical cleaning, to allow for writers' rotation. In project 4 , the main attraction is an artificial tree, a sort of sculpture, with a central clock and four branches, each colored with seasonal vegetation, indicating four different paths corresponding to the seasons and their emotional atmospheres. Luca ${ }^{2}$ - a student from team 4 - explains: "If you feel sad, maybe for a bad mark at school, you can walk the winter path; but if you are happy, you go for the spring one!"

When the time comes to answer director Benhadj's second question about emotions and surprises, excitement increases: nobody wants to give up telling their experiences. Keywords in the narratives are: expectations, satisfaction, freedom, and team work. Several students underline how they did not expect to experience such intense satisfaction in working together. Pointing to their drawing, visibly excited, Carla from team 3, claims: "I didn't imagine we could do something like this. . . now I see it! I think it's very original."

The blackboard with its significant size has made everyone's work visible in real time: a multiplier of satisfaction, creating opportunities for feedback, expanding the meaning of "audience." The idea of satisfaction is expressed by students in many ways: "to see what you just did and realize that everybody looks at it" (Luisa), "to know that before there was nothing and now. . look here!" (Angela), "to understand that maybe we will be able to change something with our drawings" (Oscar), "to work so freely in cooperation and share the product" (Eleonora).

More than just simple satisfaction for the work done emerges here. Students overcome the dichotomy between individual and collective approaches to learning, clearly showing the contribution of the TLA to socio-materiality. Productive participation in knowledge creation processes needs the transformation of personal contributions toward the

${ }^{2}$ Student names have been changed to protect their privacy. construction of collective products that "embody" the shared enterprise. Our students are involved in such creative processes; therefore, their individual contributions are intertwined in social processes.

\section{A Critical Incident: Re-shaping Social Practices}

In the "words room" session, several "critical incidents" occurred, in the sense indicated by Tripp $(1993,2006)$ : events that produce new interpretations and allow their significance to be unraveled. We focus on an emblematic example: the case of Marco, a clever but difficult student from team 2. When students are invited to stop drawing, Marco furtively takes a chalk, quickly sketches a little circle under his team's drawing, and writes something inside it, confusedly. He looks around with a somewhat guilty expression, almost waiting for a reproach for not putting aside the chalk. One of the teachers asks him: "What were you in such a hurry to write?" Surprised by this attention, lacking any punitive intention, he replies: "I wrote: this is for you from us."

Marco feels entitled to act, breaking the order given (putting aside the chalk), probably because of the new setting. The large blackboard is a material space inviting to be filled. Even the teacher reacts in an unexpected way: she asks for the reason of such behavior instead of reproaching Marco. The setting elicited new social practices from both the teacher and the student, allowing the discovery of Marco's awareness of having achieved something that deserves to be offered to others. Both teachers are astonished at the involvement transpiring from the words of this challenging student.

The TLA posits that by solving complex, "authentic," and challenging problems, social practices are re-negotiated based on the contamination offered by entering new settings and using flexible tools. This is exactly what happened in our case. This experience created the space for new ways of interacting, for both teachers and students. Crossing boundaries between settings school and museum - represents a crucial experience to review the practices supporting the creation of objects, such as how to react when a student does not follow the teacher's indications.

\section{DISCUSSION AND FINAL REMARKS}

In this research, we have tried to explore how learning and teaching change when located in an alternative place. Our theoretical lens, in particular the TLA approach, allows us to understand the learning context as a triadic relationship between learners, teachers, and objects. Since the relationship between socio-materiality and the TLA needs to be further explored, we have provided some empirical evidence of their connections. Indeed, the MACRO-ASILO's "words room" has proved to be a rich space, creatively challenging students and putting teachers and students in a novel situation. A typical school setting, which traditionally enhances top-down interactions, has now become a space for all through the huge blackboard, where unexpected processes occur and productivity flourishes, creating an impact on students' ideational processes and their performance. Students have explored all of its potential, positioning themselves - both physically and cognitively - in different ways to draw, discuss, 
and observe, making their emotions more alive. As shown elsewhere (Cattaruzza et al., 2019), the space with its objects becomes part of the interactive actions. All participants, including non-traditional school actors - director Benhadj in our case form a virtuous triangulation, where each element enriches the other. In this sense, the contraposition between Piaget and Vygotskij is overcome: knowledge into the objects and knowledge possessed by human actors compose a complex polyphony, made by many types of "voices" and different rhythms (Bakhtin, 1981).

Even research conducted in non-school contexts (Kumpulainen et al., 2014; Rajala and Akkerman, 2019; Yrjönsuuri et al., 2019) has shown how objects participate actively in shaping the learning process. Similarly, we found that students' engagement improves greatly, and it goes beyond learning concepts so that collaborative and creative knowledge building is possible. When students are challenged to produce useful objects for a large community, they feel part of this community becoming active citizens - and feel entitled to improve it.

Using a large blackboard and moving furniture, students have had the chance to work together, experiencing their mutual influence and the impact of cooperation in real time, together with a sense of self-efficacy (Bandura, 2010). Learning is now not only connected to the possibility to build knowledge, but it emerges from the deep engagement elicited in the continuous shift from presenters to audience: questionanswer processes were intense, and new interpretations of traditional solutions arose, encouraging creative developments. The triangulation learners-teachers/expert-object was activated by the new "place" where objects composing the setting (the blackboard, the chalk, the cameras, and the other technological means) functioned as mediators to build a new common object: the imagined square. Moreover, the meaning of the various dimensions tackled by the project was exploited, and the museum has offered a place where learning means "giving body" to ideas, concepts, and social interactions.

We witnessed how materiality implies also the interconnection between different time-space levels. One level is the local context in which students are working, in our case, the museum. The other levels concern the contexts evoked; one could be the physical square visited and studied by the students and/or the imagined square they were planning. Another level pertains to the classroom, where a large part of the preparatory work was done.

\section{REFERENCES}

Anderson, J. R., Reder, L. M., and Simon, H. A. (1996). Situated learning and education. Educ. Res. 25, 5-11.

Bakhtin, M. (1981). The Dialogic Imagination. Four Essays by M. M. Bakhtin. Austin: University of Texas Press.

Bandura, A. (2010). "Self-efficacy," The Corsini Encyclopedia of Psychology, eds C. Nemeroff, and W. E. Craighead (Hoboken, NJ: John Wiley \& Sons), $1-3$.

Barad, K. (2003). Posthumanist performativity: toward an understanding of how matter comes to matter. Signs 28, 801-831. doi: 10.1086/ 345321
As Säljö (2019) contends, instruments are tools meant not only to build objects but also to think with and through them. So, the target object - the square in our case - becomes an additional material object to reach new cognitive levels where many points of view may interweave. This leads to further levels, which in our case concern the symbolic value attached to the object. These values are constructed through various discourses and representations of the object. The square, therefore, becomes an agora to think, a space to meet, a venue for art, a central hub for business, and a destination and point of departure.

In conclusion, in this experience, learning is a process that is deeply affected by the space and place in which it occurs and by the materials available. Such materiality has a multi-level dimension where each level enriches the other and all together influence the learning outcomes.

\section{DATA AVAILABILITY STATEMENT}

All datasets generated for this study are included in the article/Supplementary Material.

\section{ETHICS STATEMENT}

Ethical review and approval was not required for the study on human participants in accordance with the local legislation and institutional requirements. Written informed consent from the participants' legal guardian/next of kin was not required to participate in this study in accordance with the national legislation and the institutional requirements.

\section{AUTHOR CONTRIBUTIONS}

The authors have shared the responsibility for the theoretical framework, data collection, documentation, and writing.

\section{SUPPLEMENTARY MATERIAL}

The Supplementary Material for this article can be found online at: https://www.frontiersin.org/articles/10.3389/fpsyg. 2020.00943/full\#supplementary-material

Barzanò, G., and Grimaldi, E. (2013). Discourses of merit. The hot potato of teacher evaluation in Italy. J. Educ. Policy 28, 767-791. doi: 10.1080/02680939.2013. 774439

Bellanca, J. A. (ed.) (2010). 21st Century Skills: Rethinking How Students Learn. Bloomington, IN: Solution Tree Press.

Bruner, J. S. (1996). The Culture of Education. Cambridge, MA: Harvard University Press.

Cattaruzza, E., Ligorio, M. B., and Iannaccone, A. (2019). Sociomateriality as a partner in the polyphony of students positioning. Learn. Cult. Soc. Interact. 22:100332. doi: 10.1016/j.lcsi.2019.100332

Cobb, P., and Bowers, J. (1999). Cognitive and situated learning perspectives in theory and practice. Educ. Res. 28, 4-15. doi: 10.3102/0013189x028002004 
Cole, M. (1998). Cultural Psychology: A Once and Future Discipline. Cambridge, MA: Harvard University Press.

Denzin, N. K. (2001). Interpretive Interactionism. London: SAGE.

Engeström, Y. (1999). "Activity theory and individual and social transformation," in Learning in Doing: Social, Cognitive, and Computational Perspectives. Perspectives on Activity Theory, Y. Engeström, R. Miettinen, and R.-L. Punamäki (Cambridge: Cambridge University Press).

Hakkarainen, K. (2009). A knowledge-practice perspective on technologymediated learning. Int. J. Comput. Support. Collab. Learn. 4, 213-231. doi: 10.1007/s11412-009-9064-x

Hallgarten, J., Hannon, V., and Beresford, T. (2015). Creative Public Leadership: How School System Leaders Can Create the Conditions for System-wide Innovation. Dubai: WISE.

Johri, A. (2011). The socio-materiality of learning practices and implications for the field of learning technology. Res. Learn. Technol. 19, 207-217.

Karlgren, K., Paavola, S., and Ligorio, M. B. (2020). Introduction: what are knowledge work practices in education? How can we study and promote them? Res. Papers Educ. 35, 1-7. doi: 10.1080/02671522.2019.1677761

Kober, N. (2015). Reaching Students: What Research Says ABOUT EFFECTIVE INSTRUCTIon in Undergraduate Science and Engineering. Washington, DC: National Academies Press.

Kumpulainen, K., Mikkola, A., and Jaatinen, A. M. (2014). The chronotopes of technology-mediated creative learning practices in an elementary school community. Learn. Media Technol. 39, 53-74. doi: 10.1080/17439884.2012. 752383

Latour, B. (2005). Reassembling the Social: An Introduction to Actor-NetworkTheory. Oxford: Oxford University Press.

Mayorga, C. R. (2019). Materiality and immateriality in based-object pedagogies at the Ure Museum of Greek Archaeology. Anu. Filol. Antiqua Med. 1, 52-58.

Mirza, N. M. (2016). Emotions, development and materiality at school: a culturalhistorical approach. Integrat. Psychol. Behav. Sci. 50, 634-654. doi: 10.1007/ s12124-016-9348-4

Nielsen, J. A. (2015). Assessment of innovation competency: a thematic analysis of upper secondary school teachers' talk. J. Educ. Res. 108, 318-330. doi: 10.1080/00220671.2014.886178

Orlikowski, W. J., and Scott, S. V. (2008). Sociomateriality: Challenging the separation of technology, work and organization. Ann. Acad. of Manag. 2, 433-474. doi: 10.1080/19416520802211644

Paavola, S., and Hakkarainen, K. (2014). Trialogical Approach for Knowledge Creation. In Knowledge Creation in Education. Singapore: Springer, 53-73.

Piaget, J., and Inhelder, B. (1967). "See especially "systems of reference and horizontal-vertical coordinates," in The Child's Conception of Space, Ed. J. Piaget (New York, NY: W. W. Norton \&Co), 375-418.
Rajala, A., and Akkerman, S. F. (2019). Researching reinterpretations of educational activity in dialogic interactions during a fieldtrip. Learn. Cult. Soc. Interact. 20, 32-44. doi: 10.1016/j.lcsi.2017.10.006

Reimers, F. (2009). "Educating for global competency," in International Perspectives on the Goals of Universal Basic and Secondary Education, eds J. Cohen and M. Malin (Abingdon-on-Thames, UK: Routledge), 183-202.

Reimers, F. M., Chopra, V., Chung, C. K., Higdon, J., and O’Donnell, E. B. (2016). Empowering Global Citizens. North Charleston; South Carolina: CreateSpace Independent Publishing Platform.

Ritella, G., Ligorio, M. B., and Hakkarainen, K. (2016). Theorizing space-time relations in education: the concept of chronotope. Frontl. Learn. Res. 4, 48-55. doi: $10.14786 /$ flr.v4i4.210

Säljö, R. (2019). "Materiality, learning, and cognitive practices: Artifacts as instruments of thinking," in Emergent Practices and Material Conditions in Learning and Teaching With Technologies (Cham: Springer), 21-32.

Sansone, N., Cesareni, D., and Ligorio, M. B. (2016). Il Trialogical Learning Approach per rinnovare la didattica. Ital. J. Educ. Technol. 24, 82-82.

Sobe, N. W. (2012) “Cosmopolitan education," in Handbook of Cosmopolitan Studies, ed G. Delanty (New York, NY: Routledge), 268-276.

Sørensen, E. (2009). The Materiality of Learning: Technology and Knowledge in Educational Practice. Cambridge: Cambridge University Press.

Spelke, E. S. (1991). "Physical knowledge in infancy: reflections on Piaget's theory," in The Epigenesis of Mind: Essays on Biology and Cognition, eds R. Gelman, and S. Carey (Abingdon: Psychology Press), 133-169.

Tripp, D. (1993). Critical Incidents in Teaching. Abingdon: Routledge.

Tripp, D. (2006). Teachers' lives, critical incidents, and professional practice. Int. J. Qual. Stud. Educ. 7, 65-76. doi: 10.1080/0951839940070105

Vygotskij, L. S. (1978). Mind in Society. Cambridge, MA: Harvard University Press. Yrjönsuuri, V., Kangas, K., and Seitamaa-Hakkarainen, P. (2019). "Material objects as tools for organizing collaboration in maker-centered learning," in Proceedings of the FabLearn Europe 2019 Conference, London, 1-3.

Conflict of Interest: The authors declare that the research was conducted in the absence of any commercial or financial relationships that could be construed as a potential conflict of interest.

Copyright (C) 2020 Barzanò, Amenduni, Cutello, Lissoni, Pecorelli, Quarta, Raffio, Regazzini, Zacchilli and Ligorio. This is an open-access article distributed under the terms of the Creative Commons Attribution License (CC BY). The use, distribution or reproduction in other forums is permitted, provided the original author(s) and the copyright owner(s) are credited and that the original publication in this journal is cited, in accordance with accepted academic practice. No use, distribution or reproduction is permitted which does not comply with these terms. 


\title{
Objects as Communicative Mediators in Children With Autism Spectrum Disorder
}

\author{
Federico Manzi ${ }^{1 *}$, Giulia Savarese ${ }^{2 *}$, Monica Mollo $^{3}$ and Antonio lannaccone ${ }^{4}$ \\ ${ }^{1}$ Research Unit on Theory of Mind, Department of Psychology, Università Cattolica del Sacro Cuore, Milan, Italy, \\ ${ }^{2}$ Department of Medicine, Surgery and Dentistry, University of Salerno, Salerno, Italy, ${ }^{3}$ Department of Human, Philosophical, \\ and Education Sciences, University of Salerno, Salerno, Italy, ${ }^{4}$ Institut de Psychologie et Éducation, Université de Neuchâtel, \\ Neuchâtel, Switzerland
}

In recent years, the socio-material perspective has informed an important interdisciplinary debate concerning the role of the physical world (i.e., the objects) in human psychological development. Several studies in the field of developmental psychology showed positive achievements in explaining the relationship between the subject and the social context through a socio-material approach, in particular in the early development. The importance of objects was also recognized in children with autism spectrum disorder (ASD), showing that these children are characterized by alterations in the use of the objects from early development. Some studies highlighted

OPEN ACCESS

Edited by: Martina Smorti, University of Pisa, Italy

Reviewed by:

Giuliana Pinto,

University of Florence, Italy

Claudio Longobardi,

University of Turin, Italy

*Correspondence:

Federico Manzi federico.manzi@unicatt.it

Giulia Savarese

gsavarese@unisa.it

Specialty section: This article was submitted to Developmental Psychology, a section of the journal Frontiers in Psychology

Received: 06 February 2020 Accepted: 14 May 2020

Published: 17 June 2020

Citation:

Manzi F, Savarese G, Mollo M and lannaccone $A$ (2020) Objects as Communicative Mediators in Children With Autism Spectrum Disorder. Front. Psychol. 11:1269 doi: 10.3389/fpsyg.2020.01269 that objects could be a facilitator in the interactions between children with ASD and peers. However, the role of objects was not sufficiently investigated in interactions between children with ASD and adults. The main purpose of the present study was to investigate in children with ASD the communicative function that the activities with objects assume in the interactions with adults, highlighting the mediator role of objects in these interactions. More generally, this study also aims to highlight the relevance of adopting a socio-material perspective to explore some neglected aspects of the psychological activity of children with ASD. To test this hypothesis, we conducted an extensive exploratory study, collecting data from a sample of 3-year-old $(N=18 ; F=3)$ and 4-year-old ( $N=26 ; F=3$ ) with ASD. Children were observed in a free-play situation with an adult. They were free to choose an object from a predefined set. Through quantitative data, we have described the general characteristics of the manipulation of objects; through qualitative data, we aimed to capture and describe, in microgenetic sequences, some characteristics of children's activities, defined as socio-material. The analysis of the socio-material activities suggested the role of objects as mediator of the interactions between children with ASD and adults.

Keywords: autistic children, socio-material perspective, object use, communicative mediators, children play

\section{THEORETICAL FRAME}

The socio-material perspective emphasizes the role of both social and material dimensions of artifacts - conceived as closely interrelated - in psychological activity and investigates which features of artifacts can affect children's social interaction patterns. Several studies have highlighted the role of objects in the very early stages of psychological development, both in allowing the 
expansion of psychological activity and as regulators of communication between social partners (Iannaccone, 2015; Moreno-Núñez et al., 2017; Manzi, 2018; Cattaruzza, 2019; Cattaruzza et al., 2019a,b).

The field of investigation of the role of objects in psychology is very broad and informed by the contributions of various theoretical perspectives and empirical approaches. Among others, Piaget, Vygotsky and Moscovici have tried to understand in very intriguing ways the role of objects in human development. In his work, Piaget (1928, 1952a, 1954, 1962, 1972) emphasizes how children actively build their own cognitive worlds, organizing knowledge into patterns, or mental structures, that serve to represent the reality to which they must adapt. Piaget's approach underlines the interaction between the individual and their environment, without clearly overcoming the subjectobject dualism, as the socio-material approach advocates:

A child sometimes sucks his thumb as early as the second month, grabs objects at around 4-5 months, then shakes them, swings them, rubs them and finally learns to throw them and catch them. These behaviors presuppose at least two poles: on the one hand is accommodation, since it is necessary to adjust movements and perceptions to the objects themselves, and on the other hand is assimilation of objects to one's own activity, since the child is not interested in the object in itself, but in so far as it can serve as "food" for a previous behavior or in the process of being acquired. This assimilation of the reality with sensory-motor schemes presents itself in two complementary aspects: it is an active repetition and consolidation (hence the "circular reaction" described by Baldwin), (...) it is "mental digestion," that is to say perception or conception of the object according to its incorporation into a real or possible action: (...) In this regard, it is obvious that this double function of assimilation is only one in concrete activity, because it is to the extent that the subject repeats his behaviors by reproductive assimilation that he assimilates objects to actions and that these become by this very fact patterns. These schemas then constitute the functional equivalent of concepts and subsequent logical relations. (Piaget, 1952b, p. 15, par. 5).

However, despite the dualistic position, Piaget's works made essential contributions to understand the relationship between cognitive development and physical reality. For example, the micro-genetic method has allowed for comprehending how we can provoke and observe the transformations of this relationship between the subject and the physical world in a limited space-time sequence. This is a fundamental methodological condition for understanding the development of materiality in child development.

Vygotsky (2004), while sharing Piaget's view of the child as an active builder of their own knowledge, highlights that cognitive development largely depends on the progressive appropriation of psychological tools made available by society and produced by culture (Ben-Ari and Kedem-Friedrich, 2000). Moreover, he argues that the transition from practical intelligence, interrelated to reality, to a more abstract intelligence (shared and sharable) is mediated by cultural artifacts, mainly language. Vygotskjian's approach conceives artifacts as symbolic mediators (both material and psychological) of human social cognition and as products of historical and cultural development. These are used by humans to interact with others and reality, and to reflect on their activities. In this sense, objects, as artifacts (and largely socio-material ones), represent a constitutive component of human life on two different levels: at an interpersonal level, these "tools" regulate communication, interaction and all social activities; at an intrapersonal level, they extend human functions and, once internalized, guide thought (Moro, 2011). In these terms, the psychological activity with and the awareness of artifacts depend on the nature of the interaction between the human and non-human components of this dialectical dyad. As well-explained by Vygotsky (see quote below), a child's play, involving both above-mentioned components, is a situation that allows them to explore the physical and social world (Iannaccone et al., 2019). In this perspective, a child's play does not represent a simple reproduction of the experience but a creative reworking of it:

A child's play very often is just an echo of what he saw and heard adults do; nevertheless, these elements of his previous experience are never merely reproduced in play in exactly the way they occurred in reality. A child's play is not simply a reproduction of what he has experienced, but a creative reworking of the impressions he has acquired. He combines them and uses them to construct a new reality, one that conforms to his own needs and desires. Children's desire to draw and make up stories are other examples of exactly this same type of imagination and play. (Vygotskij, 1976, p. 11).

Also, social psychology has provided some interesting contributions to conceptualizing the notion of objects and especially the mediating role that the partner assumes in the interaction. In this regard, Moscovici (1976), assuming a broad psychosocial perspective, adopts a triadic model. The key aspect of Moscovici's theory is represented by a ternary reading of facts and relations, replacing the typical binary approach of the two terms of subject and object with one of three terms: the individual subject (I), the social subject (Other) and the Object. The I-Object relationship is always mediated by the Other; it can take a static form as "co-presence" or a dynamic form as "interaction," and can lead to changes in thinking and behavior. Although this approach assumes a continuous interchange between the three elements of the triadic relationship, the dialogical theories provide an important enhancement to the understanding of materiality. Linell (2009), adopting a dialogical approach, considers human interactions impossible without the presence of objects, considered as artifacts that embody the cultural heritage of the human species (see also Tomasello, 2016). In fact, as Linell (2009) states, "many forms of human cognition and communication cannot occur without artifacts" (p. 345). From the dialogical approach, human interactions are characterized by combined actions during which humans using objects to transform their perspectives of the interactive world (Linell, 2009).

According to this point of view, mental activity cannot be considered as a "decontextualized" activity "in solitude" but it largely depends on the continuous interaction of individuals with the physical and social world (Clark and Chalmers, 1998). The process of thinking can be considered as a social co-construction of the meaning of the social experiences that continuously 
involve humans (Perret-Clermont, 2004; Iannaccone and Bruner, 2010; Iannaccone et al., 2016). Thus, thinking is a "form of social practice" (Radford, 2003) that refers to lived experience in concrete situations. Based on these assumptions, thinking activities are triggered by actions in a specific socio-material activity context: these activities individually, isolated from their contexts, have no meaning (Zucchermaglio, 1996; Ligorio, 2010; Coppola et al., 2019). The above considerations allow for defining the main assumption of the socio-material perspective: human development is intrinsically bound to the material components of the social context.

The attention to the role of objects in children's social interactions is also addressed in the research field of developmental psychology. Pioneering studies have shown that objects can support children's interactions in early development (Jacobson, 1981; De Stefano and Muller, 1982; Lieber and Beckman, 1991) and stimulate more complex interactions among children (De Stefano and Muller, 1982). The importance of objects in children was also observed in interactions between children and adults, in which the children's use of objects changes as a function of the sociocultural background of the caregivers, who transmit this heritage (Bakeman et al., 1990; Tomasello et al., 1990). Actually, the role of objects in children's interactions-both with peers and adults-is even more important considering also the recent design of new relational artifacts (Turkle, 2004), i.e., robots (Manzi et al., 2017, 2020; Marchetti et al., 2018, 2020; Di Dio et al., 2019, 2020a,b; Manzi et al., 2020). Recently, several studies have highlighted the role (and uses) of objects, as mediators, in the adult-child interactions in early development (Rodríguez and Moro, 1998; Dimitrova and Moro, 2013; Rodríguez et al., 2015; MorenoNúñez et al., 2017). In these interactions, adults acquire the role of the scaffolder, teaching the child the different (conventional) uses of the object (Moro, 2011, 2014). In Vygotskian terms, adult-child activities are part of a process of the co-construction of knowledge that involves a negotiation of intersubjective meanings. In this sense, the object represents a support in the interactions between child and adult, becoming one of the components of secondary intersubjectivity (Trevarthen and Hubley, 1978). This leads to a decisive transformation of the children's interaction: from a dyadic interaction (child-object or child-adult) to a triadic interaction (adult-child-object). In typical development, it is clear how the use of objects represents a crucial element of interactions (Barthélémy-Musso et al., 2013; Rodríguez et al., 2015).

\section{The Object in Autism Spectrum Disorder}

In the previous paragraph, we delineated some fundamental theoretical coordinates to define the importance that objects have in children's social interactions, particularly in typical development. In the present paragraph, we will briefly outline the importance of studying the role of objects for children with autism spectrum disorder (ASD). As described by DSM 5, autism is a persistent deficit in communication and social interaction that manifests itself in various contexts (DSM, 5). Additionally, autism is characterized by restricted and/or repetitive behavior patterns, interests or activities: in this sense, children's modalities of the use of objects represent an important element to be considered in the diagnosis. It is not intended here to analyze the children's use of objects as a diagnostic factor, but only to highlight that the objects represent an element of diagnostic interest. With respect to the use of objects, Kanner (1943) was among the first to note that, despite differences and limitations, children with ASD exhibit a particular interest in objects. Generally, children with ASD present altered patterns of object exploration and manipulation starting from an early stage of development (Sigman and Ungerer, 1984; Bruckner and Yoder, 2007; Mottron et al., 2007; Ozonoff et al., 2008; for a review see Williams et al., 1999). Furthermore, several pieces of research have shown alterations related to the conventional use of objects, namely the appropriate use of everyday objects (Lord, 1983; Loveland and Tunali, 1991; Bachevalier, 1994; Williams et al., 2005). In addition, the use of objects in children with ASD has been extensively studied in functional and symbolic play, showing alterations to use them also in play (Jarrold et al., 1993; Jarrold, 2003). However, other studies have identified how objects can become mediators of interactions between children with ASD and peers (Romanczyk and Goren, 1975; Lord, 1983, 1984; Lord and Hopkins, 1986). Thus, the objects with a communicative function in the interactions between children with ASD and peers has been recognized in literature, although not further investigated. However, no study has ever specifically analyzed 310 the role of objects as mediators of interactions between children with ASD and adults in a socio-material perspective.

\section{Aims}

The main purpose of the present study was to investigate in children with ASD the communicative function that the activities with objects assume in the interactions with adults, highlighting the mediator role of objects in these interactions. To fulfill this aim, we implemented a quasi-experimental design, observing different forms of children's "playful" interactions. A broader aim was to provide insights in adopting a sociomaterial approach to the activities of children with ASD analyzing the wider context.

\section{MATERIALS AND METHODS}

\section{Participants}

Forty-four (44) Italian preschool-age children with ASD participated in the experiment. The children were divided into two age groups as follows: 3 -year-olds $(N=18, F=7 ; M=32.94$, $S E=4.13)$ and 4-year-olds $(N=26, F=10 ; M=48.36, S E=5.42)$. The children were recruited from different rehabilitation centers of the Campania region, Italy. Inclusion criteria for the two groups are related to the child's diagnosis of ASD, according to the Diagnostic and Statistical Manual of Mental Disorders criteria (DSM 5: American Psychiatric Association [APA], 2013) made by experts. The children's parents received a written explanation of the procedure of the study, the measurement items and the materials used, and they gave written consent. The number of participants correspond with the number of children recruited. 


\section{Measures}

\section{Socio-Material Use of Objects Checklist}

The "Socio-Material Use of Objects" checklist (SMUO; Savarese et al., 2017; Iannaccone et al., 2018) consists of 14 items (see Appendix 1). The items explore both the social behaviors of the child toward an interactive partner and the activities displayed toward the objects. Thus, the checklist focuses on how the child "behaves" with the object within an interaction with a partner. For each item, the observer assigned score 1 when the behavior or activity occurred. The sum of the 14 items is grouped into a factor named Social Modalities, which ranges from 0 to 14 . This score allows for estimating children's social interaction patterns in a context involving an object and a partner. In addition, the observer has to fill an observational section to provide detailed information concerning the events occurring during the play session.

According to the socio-material perspective and the scientific literature (Dominguez et al., 2006; Bruckner and Yoder, 2007), children's activities with objects were classified according to three criteria: (1) Sensory-Motor Activities (SMAs), typical of interactions in which the child uses the toy as a means to engage in a sensorial experience (involving touch, hearing, sight, smell and/or taste), including any self-stimulating behavior with repetition of gestures or specific uses of an object (stacking, piling or slamming to hear a noise); (2) Canonical Activities (Cas), referring to using the functional characteristics of objects and uses encoded in the child's past experience; (3) Social-Interactive Activities (SIAs), referring to the child that uses the object as a mediator tool that promotes the relationship with the adult.

\section{Procedure}

The study involved two experimenters (the observers) and one experienced educator (i.e., the adult) who were qualified to work with children with ASD and to observe them in different interactive contexts. Two observers were involved for each child, allowing for a comparison between the two sets of observations.

The experienced educator introduced the children to a set of objects (toy cars, dolls, plasticine, cubes etc.) and they were free to choose their preferred object during the interaction. The materials were selected considering recommendations from previous studies showing potential preferences of the types of objects by children with ASD (Williams et al., 1999; Ziviani et al., 2001; Dominguez et al., 2006). Specifically, children could choose from objects that potentially elicited different types of play behavior (e.g., sensorimotor, canonical, symbolic). Both experimenters completed the checklist, verifying the presence of social behavior toward the adult and activities with the object during the play session. In addition, the two experimenters had to independently note what had occurred during the play session. The observational comments were also enriched with the considerations of the experienced educator at the end of each session. The observation of the free play session lasted about 10 minutes and was carried out in quiet rooms in different motor rehabilitation centers in the region of Campania, Italy.

\section{RESULTS}

\section{Analysis of Children's Social Modalities}

In addition to the diagnosis of autism provided by experts, SMUO has been used to assess both the social behavior exhibited by children toward their partner and their exploratory behavior toward the objects. The checklist allowed for a general score of the children's social interaction modalities.

The ANOVA analysis of the mean score of Social Modalities of interaction shows a significant difference between 3- and 4year-old children (3 years: $N=18, M=1.11, S E=0.75$; 4 years: $N=26, M=1.85, S E=1.05, p<0.05)$. Compared to the 3 year-old children, the 4 -year-olds had a higher mean score and exhibited more social behaviors. Although this data seems to indicate an effect of age on children's modalities of interactions, this score is not a diagnostic index of the severity of the pathology, so it is purely informative with respect to our sample of a greater presence of interactive behaviors in the older children's group.

\section{Analysis of Children's Activities With the Objects}

As mentioned above, children's activities were classified in three different type of activities (SMAs, Cas and SIAs) (for details see section "Measures"). Two independent judges evaluated the children's activities. The inter-rater reliability scores were substantial (Cronbach's Alpha $=0.71$ ).

A Chi-square analysis (Table 1) did not reveal any differences in the type of activities between the 3- and 4-year-old children. The frequencies of the three categories observed refer to the number of participants. However, the 3-year-old children had a greater tendency to engage in SMAs (61.1\%), while the 4-yearold children tended to engage in SIAs (34.6\%) and SMAs (42.3\%). The Cas frequencies were similar for both the 3-and 4-year-old children. Although children are of different ages, both groups (including the older ones) have typical ASD difficulties in the activities with objects. However, there is an increase in activities involving the Other as a function of age, although this increase is not significant.

\section{Qualitative Data: Observations of the Children's Activities}

Qualitative data were obtained from microgenetic observations of children's activities aiming at identifying the occurrence of behaviors that indicated the type of activity that the child performed with the object within the context of playful interactions with the objects. Specifically, the aim of the analysis

TABLE 1 | Distribution of activity types for 3- and 4-year-old children.

\begin{tabular}{|c|c|c|c|}
\hline & 3 years old $\mathrm{N}(\%)$ & 4 years old $N(\%)$ & 3 years vs. 4 years sign $(p)$ \\
\hline SMAs & $11(61.1)$ & $11(42.3)$ & ns \\
\hline CAs & $5(27.8)$ & $6(23.1)$ & ns \\
\hline SIAs & $2(11.1)$ & $9(34.6)$ & ns \\
\hline
\end{tabular}


of the qualitative data was to search for evidence on the use of objects as mediators in the social interaction between ASD children and adults.

Qualitative observations will be presented to provide examples of the different types of activities (Sensory-Motor Activities, Canonical Activities, and Social-Interactive Activities) in the play context. As mentioned above, in each scenario, the child freely chose the object and interacted with the adult. All of the observations were conducted in May 2019.

\section{Sensory-Motor Activities}

In Sensory-Motor Activities, the child uses the toy as a means to activate sensory channels (touch, hearing, sight, smell, taste) and for self-stimulation through the repetition of gestures and methods of using the object (stacking, piling, slamming to hear the noise, etc.).

Observation 1 - 22.02.2017 - Giovanni (32-monthold male child).

"[Giovanni] spontaneously took the object and brought it toward his face, exploring it with his sense of smell. He then continued the exploration by manipulating the object for $30 \mathrm{~s}$, after which he placed it on the ground."

The educator's commentary underlines an activity based on sensoriality, whereby the child explores the object's material characteristics.

\section{"[Giovanni] was then asked to take the object. Giovanni walked away from the operator and started walking around the room for about $30 \mathrm{~s}$. He then returned to the activity, spontaneously taking the buildings, exploring them, and resting them on the floor. The operator started to construct a tower and the child imitated his action."}

Here, the educator's commentary highlights how the child does not respond to requests to take the object and share it.

In this interesting case, the child does not seem able to respond explicitly to the adult's requests for interaction, and does not manifest linguistic behaviors of sharing. Nevertheless, the educator's action of building a tower gives the child the opportunity to start an imitative action. In one sense, construction constitutes the socio-material element of the situation, allowing the child to share the realization of the task (albeit limited to the "remote" coordination of actions required by imitative conduct) and to somehow mediate the communicative function with the adult.

Observation 2 - 23.02.2017 - Luigi (48-month-old male child).

Luigi chose as an object a series of cubes made of a hard plastic material and with a concave space on one of its faces.

\footnotetext{
"Luigi spontaneously begins to share attention with the aim of reaching the object, which is beyond his reach. He plays properly only with large construction cubes, which he manages to stack on a model."
}

A profile is outlined in which Luigi manages to make imitations but does not present shared attention or pointing.

"[When left to play with the cubes, Luigi] shows an absorbent interest in the part underneath the cubes that is concave and where he usually puts his fingers [...] the actions carried out by Luigi with the cubes include scattering, heaping, putting in a row, or overlapping. If stressed (about three times), the child returns to stack."

This observation indicates the child's exploration of the object's material characteristics, focussing only on the concave part of the cubes.

In the observation of Giovanni, it is interesting to observe how the object's characteristics constitute real affordances that invite the child to perform specific sequences of actions, the nature of which obviously depends on the degree of psychological development and the severity of the autistic pathology. In the observation of Luigi, the interaction with the object constitutes an interesting element that highlights the child's ability to act.

Observation 3 - 24.02.2017 - Francesco (60-month-old male child).

"[Francesco] sniffs and visually chases soft rubber balls while sliding
down... he has several balls available but chooses to always use the
same ball."

The educator's observation underlines the particular sensorial interests shown by the child. It is noteworthy that attempts to involve the operator or others in the game are absent.

Observation 4 - 25.02.2017 - Cosimo (48-month-old male child).

The toy chosen by Cosimo is an action figure. During the $120 \mathrm{~s}$ of observation:

"[Cosimo] grabs the doll, shakes it, puts it in his mouth and then places it down, picks it up and shakes it again by rotating it in his hand, first to the right and then to the left, and walks simultaneously around the room with fast movements. He removes the hat from the doll's head and returns to shaking only the hat first and then the rest of the toy with both hands. He puts the hat on the ground and shakes only the rest of the doll, gets up and sits down immediately afterward, takes other toys similar to the one in his hands, disassembles them and takes only the hat... in his hands he has two hats of two dolls, and he puts them in a row and looks at them."

The mouth, hands and eyes are the sensory channels that orientate Cosimo in his use of the toys, together with the repetition of some movements such as shaking the toys, their positioning in space and the noise they make.

\section{"Cosimo puts the dolls inside the container while shaking it to make a loud noise."}

Both observing educators report the absence of attempts by the child to involve others in the game. Their accounts describe another interesting aspect of the use of the object that can provide interesting information about their cognitive activity. The object manipulated by Cosimo is a complex object, consisting of multiple elements. The observed manipulation shows how the child takes this complexity into account and lets his actions be guided by the object's physical characteristics. Of course, it is impossible to deduce from the observational data the psychodynamic elements inducing the child to disassemble and reassemble the doll. These aspects, which are also of great 
interest in understanding the child's psychological life, should be interpreted using paradigms of affective psychology.

\section{Canonical Activities}

Children engaging in Canonical Activities use the toy according to its extrinsic functions and insert it into the context of external reality.

Observation 5 - 26.02.2017 - Carlo (36-month-old male child). Carlo chose a toy car made of hard plastic and featuring wheels that turn.

"Carlo has a good understanding of the object of observation. In fact, he uses the toy car on the slide, looking at how it moves. The child's observation of the interaction with the object lasts 5 minutes but he presents little eye-contact."

The educator's comments highlight how the child can use the combination of two objects and understand their canonical function.

\begin{abstract}
"Some difficulties were encountered with regard to shared attention and the return of the object when requested by the adult. However, Carlo has good imitative skills; in fact, he imitates the movements of the adult when he places the car on the slide."
\end{abstract}

We can see that the child has well-established imitative abilities, even if explicit social conduct is not observed.

Observation 6 - 27.02.2017 - Enzo (48-month-old male child).

Enzo chose an electric piano made of hard plastic and composed of several keys.

"Enzo took several minutes to examine the function of the various keys and imitated the behavior of the educator by switching [the piano] on and off several times during use. He pressed the keys of the piano only for a short period of time; instead, he preferred to listen to the output of the pre-recorded music and press the different keys to change the melody and volume."

These comments show that the child understands the canonical use of the electric piano, specifically how to make it produce sounds.

The notion of canonical manipulation allows us to ascertain that both Carlo and Enzo have acquired important social knowledge, at least as regards understanding the rules for the use of the object. The differences from uniquely sensory-motor activities are evident and allow for a more precise assessment of the methods the children adopt to relate to the social and material reality.

Observation 7 - 28.02.2017 - Roberto (64-month-old male child).

Roberto chose a soft wolf-shaped toy.

"Roberto puts the stuffed animal in a seated position, then takes the food and puts it in the saucepan and mixes it with a spoon. He brings the food to the wolf's mouth to feed it and then takes the food and drinks to bring them first to his own mouth and then toward the stuffed animal's mouth. He imitates the non-verbal signs of drinking and sleeping."

The educator's comments reveal a functional profile of Roberto with respect to his play. During the observation, Roberto showed his ability to combine the objects based on their specific configuration (he seated the wolf and put the kitchen tools back in their place) and based on their conventional characteristics (he brought the food closer to his mouth and to the wolf's mouth to imitate the gesture of feeding). However, the child did not directly look into the other's eyes while using the toys or when taking the objects.

Observation 8 - 01.03.2017 - Loretta (48-month-old female child).

The educator suggested that Loretta could play with a doll:

"Loretta takes a doll and says, "Look how beautiful this doll is! She has very beautiful hair... do we comb it?" Loretta responds to the educator's request by taking the doll, looking at it and caressing it. Then Loretta takes the comb from the ground, looks at it, touches it with her other hand, takes the doll's hat off and combs its hair. Then Loretta takes the comb from the ground and combs the doll's hair."

In this example, the child shows functional behavior toward the object and contextualizes the use of the doll and the objects at her disposal. She also demonstrates good imitation skills in using the object.

\section{"After the educator demonstrates, Loretta begins to imitate the movements of the comb on the hair or to repeat, "This hair is beautiful, it is a beautiful color.'”}

The cases of Roberto and Loretta offer clear examples of conduct that recognize the "canonical use" of objects, but the observed activities appear more advanced than in the previous cases in terms of interactive skills. In the observation of Roberto, the elements of the playful scene communicate with each other and social behaviors arise. In the observation of Loretta, there is even some element of direct communication with the educator accompanying the canonical manipulation of the object.

\section{Social-Interactive Activities}

In Social-Interactive Activities, the child explicitly uses the toy to enter a relationship with the adult.

Observation 9 - 02.03.2017 - Melvis (36-month-old male child).

Melvis chose a toy phone made of hard plastic and featuring four wheels.

\begin{abstract}
"Melvis moves the toy phone on its wheels; he takes the handset and passes it from one hand to the other, then he brings the phone to his ear and says "Pompo" (hello). Once the educator takes the phone and says "Hello," Melvis does the same, looking him in the eyes for a few (two) seconds."
\end{abstract}

In this sequence of actions, we can observe how the child carries out an interactive activity involving the other by using an object, even if only for a short time.

Observation 10 - 03.03.2017 - Stefano (44-month-old male child).

Stefano chose a toy truck made of hard plastic and featuring four wheels.

\footnotetext{
"Stefano pays attention to every part of the truck; he does not use the truck making repetitive movements. Stefano manipulates the truck in order to make movements related to its function (he puts a toy child in the driver's seat, attaches the trailer and pushes it). Stefano
} 


\section{holds the truck for one minute and 30 s. Stefano points and says the name of the desired object in order to get it."}

In this interaction sequence, the child clearly organizes an activity coherent with the functional opportunities offered by the object. At the same time, the child needs basic interaction with the educator to reach his goals. The object's required accessories propel the child to interact with the adult.

Observation 11 - 04.03.2017 - Federico (60-month-old male child).

Federico chose a ball as his game object.

"Federico looks at the ball in the basket and makes eye-contact with the therapist. He points at the object and says, 'Do we play ball?' Then the therapist asks, 'Who is the goalkeeper?' and Federico answers, 'You are the goalkeeper!'”

Federico then kicked the ball several times and the therapist acted as the goalkeeper. This example shows the child employing an object (the ball) to involve the educator in the game.

\section{DISCUSSION}

Starting from a previous exploratory study (Iannaccone et al., 2018), this research aimed to deepen the understanding of the socio-material contexts and, in particular, the role of objects in the psychological functioning of children with ASD. In the present study, children with ASD aged three and four were observed within a situation of free play with an object freely chosen by the child from a predefined set of objects. The child was free to include the adult or not within its activity with the object. A general result concerned the positive effects of also adopting a sociomaterial perspective to the analysis of the interactions between children with ASD and adults. Furthermore, the results showed that children preferred sensory and motor activities with objects independent of age and that older children had more sophisticated modalities of interaction than younger children. Finally, another fundamental result emerged from the analysis of the observations of the child-adult interaction: objects can be useful mediators of interaction with adults.

With respect to the more general theoretical outcome, in recent years, the socio-material perspective has informed an important interdisciplinary debate concerning the role of the physical world (i.e., the objects) in human psychological development, involving different branches of psychology (Malafouris, 2013, 2019). This perspective, also introduced in educational psychology studies, has provided an opportunity to highlight the importance of analyzing the socio-material context in which the relationships occur, including the educational ones (Iannaccone, 2015, 2017; Cattaruzza, 2018; Cattaruzza et al., 2019a,b; Iannaccone et al., 2019). This study also promotes researchers' awareness of the opportunities offered by this approach identifying the human mind and its development as an embodied, extended and distributed activity (Clark and Chalmers, 1998). Several studies in the field of developmental psychology showed positive achievements in explaining the relationship between the subject and the social context through a socio-material approach, in particular in the early development (Moro, 2011, 2014; Dimitrova and Moro, 2013; Rodríguez et al., 2015; Moreno-Núñez et al., 2017). These studies allowed for hypothesizing the adoption of the socio-material perspective in research and interventions with different pathologies and mental disorders, specifically with autism. This hypothesis arises from research that highlights how autism is characterized not only by a primary alteration in social relations but also by an alteration within the wider socio-material context. From these premises, our study provides for the first time the possibility to extend the socio-material approach, until now mainly used to explain typical development, even to atypical development. Specifically, our results concerning the objects as mediators of the relationship show that children with ASD actively use the sociomaterial context -albeit as a function of their symptomatic characteristics- to understand and explore the material and social world.

The results concerning the type of sociomaterial activity (Sensory-Motor Activities, Canonical Activities and SocioInteractive Activities) that the children with ASD preferred in the experimental situation of our study allowed for being aware of the intricate interplay between the psychological and material components in the experiences of these children. The observations of the socio-material activities of these children with objects seem to lead to a non-linear interpretation of the development of different interactional modalities established between children and objects. This result is in line with previous studies showing that children with ASD present alterations in the use of the objects from early development (Williams et al., 1999; Ozonoff et al., 2008). The type of socio-material activities with the object seem to be associated with the peculiarity of the symptomatology of each child and not so much to her/his chronological age. At the same time, certain modalities in approaching the physical world, i.e., sensorymotor activities, persist also in the occurrence of a more theoretically abstract level of sociomaterial activities (canonical and socio-interactive). Although further research is required to confirm this hypothesis, our findings seem to support that the relationship between the child and the physical world is not exclusively shaped through the evolution of his/her cognitive understanding.

The results concerning the observations, in the different situations examined, seem to confirm the above mentioned insights showing the role played by the socio-material context in shaping the interpersonal relationship. Although the observations do not provide sufficient evidence to fully support the hypothesis of a full-fledged role of mediation of materiality in the psychological processes, it is still reasonable to support it, as already highlighted in the preliminary research (Iannaccone et al., 2018). We believe that these results deepen our knowledge of the "humility of things" (Miller, 2010), allowing us to address at least partially what Malafouris rightly claimed: "We constantly think through things, actively engaging our surrounding material environment, but we rarely become explicitly aware of the action 
potential of this engagement in the shaping of our minds and brains" (Malafouris, 2013, p.7).

\section{CONCLUSION, LIMITATIONS AND FUTURE DIRECTIONS}

This study highlights how the sociomaterial perspective provides important insights on how ASD children interact with the physical and social world. In particular, our findings show that children independent of age prefer sensory-motor activities with objects. These activities also seem to persist in children displaying more abstract-level activities, i.e., canonical and sociointeractive. Finally, the results show that objects allow children to shape the relationship with their partner and, even if a preliminary hypothesis, can mediate the relationships. Overall these results provide at least two important considerations for the interventions with children with ASD: the first concerns the analysis of children's activities with objects considering the sociomaterial context of interaction, which could provide important information on children specific modalities of interacting with the physical and social world in different contexts, from the household to the therapy to the school; the second concerns the use of objects as mediators of the relationship between children and adults, specifically the objects could represent a starting point for establishing a communicative relationship based on the specific activities of the child. Finally, the analysis of children's activities with objects in their socio-material context in interaction with a partner could retrospectively provide important information for the diagnosis.

The study presents some limitations: a non-homogeneous sample, the absence of a comparison sample of typically developing children and, finally, the absence of a peer partner. For these reasons, future studies should test a homogeneous sample concerning the severity of the symptomatology and the type of sociomaterial activities observed. In addition, it will be necessary to recruit a control group to confirm if the results of this study are specific for ASD children. Finally, to verify whether the children's sociomaterial activities identified change as function of the type of the partner, it will be necessary to compare the same situations with a peer.

\section{REFERENCES}

American Psychiatric Association [APA] (2013). Diagnostic and Statistical Manual of Mental Disorders (DSM-5**). Washington, DC: Aufl. APA-Press.

Bachevalier, J. (1994). Medial temporal lobe structures and autism: a review of clinical and experimental findings. Neuropsychologia 32, 627-648. doi: 10.1016/ 0028-3932(94)90025-6

Bakeman, R., Adamson, L. B., Konner, M., and Barr, R. G. (1990). Kung infancy: the social context of object exploration. Child Dev. 61, 794-809.

Barthélémy-Musso, A., Tartas, V., and Guidetti, M. (2013). Prendre les objets et leurs usages au sérieux: approche développementale de la co-construction de conventions sémiotiques entre enfants. Psychol. Française 58, 67-88. doi: 10.1016/j.psfr.2012.10.001

Ben-Ari, R., and Kedem-Friedrich, P. (2000). Restructuring heterogeneous classes for cognitive development: social interactive perspective. Instruct. Sci. 28, $153-167$.

\section{DATA AVAILABILITY STATEMENT}

The datasets generated for this study are available on request to the corresponding author.

\section{ETHICS STATEMENT}

This study was carried out in accordance with the recommendations of Associazione Italiana di Psicologia (AIP), and all of the children's parents gave written informed consent in accordance with the Declaration of Helsinki. As there is no psychological ethics committee at the University of Salerno, the protocol was approved by an independent committee from the University's Centro di Counseling Psicologico (Psychological Counseling Centre). This external committee supervises research carried out by psychological researchers at the university.

\section{AUTHOR CONTRIBUTIONS}

FM, GS, MM, and AI conceived and designed the experiment. FM, GS, and MM conducted the experiments in the centers. GS and MM secured ethical approval. FM, GS, and AI carried out the statistical and qualitative analyses. All authors contributed to the writing of the manuscript.

\section{FUNDING}

300397POR18GEEKSAVARESE POR FESR 2014-2020 Department of Medicine, Surgery and Dentistry, University of Salerno, Salerno, Italy.

\section{ACKNOWLEDGMENTS}

We thank the following external experts of the Psychological Counseling Centre of the University of Salerno: Dr. Oreste Fasano (Psychologist), Dr. Nadia Pecoraro (Psychologist), and Dr. Luigi Curcio (Psychologist).

Bruckner, C. T., and Yoder, P. (2007). Restricted object use in young children with autism: definition and construct validity. Autism 11, 161-171. doi: 10.1177/ 1362361307075709

Cattaruzza, E. (2018). Exploring children's agency in a designed atelier: a sociomaterial perspective. Psihologija 51, 20-21.

Cattaruzza, E. (2019). A Sociomaterial Perspective for Learning: Exploring Atelier Activities. Doctoral Thesis, Université de Neuchâtel, Neuchâtel.

Cattaruzza, E., Iannaccone, A., and Arcidiacono, F. (2019a). Provoking social changes in a family-school space of activity. Psychol. Soc. 11, 33-47.

Cattaruzza, E., Ligorio, M. B., and Iannaccone, A. (2019b). Sociomateriality as a partner in the polyphony of students positioning. Learn. Cult. Soc. Int. 22:100332. doi: 10.1016/j.lcsi.2019. 100332

Clark, A., and Chalmers, D. (1998). The extended mind. Analysis 58, 7-19.

Coppola, C., Mollo, M., and Pacelli, T. (2019). The worlds' game: collective language manipulation as a space to develop logical abilities in a primary 
school classroom. Eur. J. Psychol. Educ. 34, 783-799. doi: 10.1007/s10212-0180401-1 doi: 10.1007/s10212-018-0401-1

De Stefano, C. T., and Muller, E. (1982). Environmental determinants of peer social activity in 18-month-old males. Infant Behav. Dev. 5, 175-183. doi: 10.1016/ s0163-6383(82)80026-x

Di Dio, C., Manzi, F., Peretti, G., Cangelosi, A., Harris, P. L., Massaro, D., et al. (2020a). Come i bambini pensano alla mente del robot: Il ruolo dell'attaccamento e della teoria della mente nell'attribuzione di stati mentali ad un agente robotico. Sistemi Intell. 1, 41-56.

Di Dio, C., Manzi, F., Peretti, G., Cangelosi, A., Harris, P. L., Massaro, D., et al. (2020b). Shall I trust you? From child human-robot interaction to trusting relationships. Front. Psychol. 11:469. doi: 10.3389/fpsyg.2020. 00469

Di Dio, C., Manzi, F., Itakura, S., Kanda, T., Ishiguro, H., Massaro, D., et al. (2019). It does not matter who you are: fairness in pre-schoolers interacting with human and robotic partners. Int. J. Soc. Robot. 1-15.

Dimitrova, N., and Moro, C. (2013). Common ground on object use associates with caregivers' gestures. Infant Behav. Dev. 36, 618-626. doi: 10.1016/j.infbeh.2013. 06.006

Dominguez, A., Ziviani, J., and Rodger, S. (2006). Play behaviours and play object preferences of young children with autistic disorder in a clinical play environment. Autism 10, 53-69. doi: 10.1177/1362361306062010

Iannaccone, A. (2015). "Materiality and educational psychology. Paper Presented at the Symposium Materiality and Human Development," in 16th Meeting of the International Society for Theoretical Psychology, Coventry.

Iannaccone, A. (2017). "Ėduquerpeutêtredur! Quelques notes autour de la notion de matérialitéenéducation," in Lesinteractionssocialesenclasse: Réflexions et Perspectives, eds M. Giglio and F. Arcidiacono (Berne: Peter Lang).

Iannaccone, A., and Bruner, J. S. (2010). Le Condizioni Sociali Del Pensiero: Contesti, Attività e Ricerca di Senso. Milano: Unicopli.

Iannaccone, A., Perret-Clermont, A. N., and Convertini, J. (2019). Children as investigators of Brunerian 'possible worlds'. The role of narrative scenarios in children's argumentative thinking. Integrat. Psychol. Behav. Sci. 53, 679-693. doi: 10.1007/s12124-019-09505-3

Iannaccone, A., Savarese, G., and Manzi, F. (2016). “The use of objects for autistic children: a study in Piagetian perspective and the use of construction blocks," in Poster presented at the Conference: 'XXIX Congresso Nazionale AIP - Sezione di PsicologiadelloSviluppo e dell'Educazione', Vicenza.

Iannaccone, A., Savarese, G., and Manzi, F. (2018). Object use in children with autism: building with blocks from a Piagetian perspective. Front. Educ. 3:12. doi: 10.3389/feduc.2018.00012

Jacobson, J. L. (1981). The role of inanimate objects in early peer interaction. Child Dev. 52, 618-626.

Jarrold, C. (2003). A review of research into pretend play in autism. Autism 7, 379-390. doi: 10.1177/1362361303007004004

Jarrold, C., Boucher, J., and Smith, P. (1993). Symbolic play in autism: a review. J. Autism. Dev. Disord. 23, 281-307. doi: 10.1007/bf01046221

Kanner, L. (1943). Autistic disturbances of affective contact. Nervous Child 2, $217-250$.

Lieber, J., and Beckman, P. J. (1991). The role of toys in individual and dyadic play among young children with handicaps. J. Appl. Dev. Psychol. 12, 189-203. doi: 10.1016/0193-3973(91)90011-r

Ligorio, M. B. (2010). Dialogical relationship between identity and learning. Cult. Psychol. 16, 93-107. doi: 10.1177/1354067x09353206

Linell, P. (2009). Rethinking Language, Mind, and World Dialogically: Interactional and Contextual Theories of Human Sense-Making. Charlotte, NC: Information Age Publishing, Inc.

Lord, C. (1983). "Autism and the comprehension of language," in Communication Problems in Autism, eds E. Schopler and G. B. Mesibov (New York, NY: Plenum Press), 257-281. doi: 10.1007/978-1-4757-4806-2_14

Lord, C. (1984). "The development of peer relations in children with autism," in Advances in Applied Developmental Psychology, eds F. J. Morrison, C. Lord, and D. P. Keating (New York, NY: Harcourt), 165-227.

Lord, C., and Hopkins, J. M. (1986). The social behaviour of autistic children with younger and same-age nonhandicapped peers. J. Autism. Dev. Disord. 16, 249-262. doi: 10.1007/bf0153 1658
Loveland, K. A., and Tunali, B. (1991). Social scripts for conversational interactions in autism and Down syndrome. J. Autism. Dev. Disord. 21, 177-186. doi: 10. $1007 /$ bf02284758

Malafouris, L. (2013). How Things Shape the Mind: A Theory of Material Engagement. Cambridge: MIT Press.

Malafouris, L. (2019). Understanding the effects of materiality on mental health. Br. J. Psych. Bull. 43, 195-200. doi: 10.1192/bjb.2019.7

Manzi, F. (2018). Materiality and the Construction of Intersubjectivity: HumanRobot Interaction in Typical Development and the use of the Objects in ASD Children. Doctoral Thesis, Università Cattolica del Sacro Cuore di Milano, Milano, MI.

Manzi, F., Di Dio, C., Itakura, S., Kanda, T., Ishiguro, H., Massaro, D., et al. (2020). Moral evaluation of Human and Robot interactions in Japanese preschoolers. Italy: CEUR Workshop Proceedings Conference.

Manzi, F., Massaro, D., Kanda, T., Tomita, K., Itakura, S., and Marchetti, A. (2017). "Teoria della Mente, bambini e robot: l'attribuzione di stati mentali," in Paper presented at XXX Congresso AIP Sezione di Psicologia dello Sviluppo e dell'Educazione, Messina.

Manzi, F., and Savarese, G. (2017). "Interaction with/through object and social functions in ASD children," in Abstract Book of the International Congress: Educa 2017 'Inequalities: What Contributions of the Educations for ...? ?, Hammamet, 73.

Marchetti, A., Manzi, F., Itakura, S., and Massaro, D. (2018). Theory of mind and humanoid robots from a lifespan perspective. Z. Psychol. 226, 98-109. doi: 10.1027/2151-2604/a000326

Marchetti, A., Miraglia, L., and Di Dio, C. (2020). Towards a socio-material approach to cognitive empathy in autistic spectrum disorder. Front. Psychol. 10:02965. doi: 10.3389/fpsyg.2019.02965

Miller, D. (2010). Stuff. Cambridge: Polity.

Moreno-Núñez, A., Rodríguez, C., and Del Olmo, M. J. (2017). Rhythmic ostensive gestures: How adults facilitate infants' entrance into early triadic interactions. Infant Behav. Dev. 49, 168-181. doi: 10.1016/j.infbeh.2017. 09.003

Moro, C. (2011). "Material culture, semiotics and early childhood development," in Children, Development and Education: Cultural, Historical, Anthropological Perspectives, eds M. Kontopodis, C. Wulf, and B. Fichtner (New York, NY: Springer Verlag), 57-70. doi: 10.1007/978-94-007-0243-1_4

Moro, C. (2014). "Le rôle de l'objetdans la construction de l'attention con jointe et dansl'accès aux intentions d'autrui," in Sémiotique, Culture et Développement Psychologique, eds C. Moro and N. Muller Mirza (Lille, FR: Presses Universitaires du Septentrion), 55-77.

Moscovici, S. (1976). La Psychanalyse: Son Image et Son Public [Psychoanalysis: Its image and its public]. Oxford: U France Press.

Mottron, L., Mineau, S., Martel, G., Bernier, C. S. C., Berthiaume, C., Dawson, M., et al. (2007). Lateral glances toward moving stimuli among young children with autism: early regulation of locally oriented perception? Dev. Psychopathol. 19, 23-36.

Ozonoff, S., Macari, S., Young, G. S., Goldring, S., Thompson, M., and Rogers, S. J. (2008). Atypical object exploration at 12 months of age is associated with autism in a prospective sample. Autism 12, 457-472. doi: 10.1177/1362361308096402

Perret-Clermont, A.-N. (2004). "The thinking spaces of the young," in Joining Society: Social Interactions and Learning in Adolescence and Youth, eds A.-N. Perret-Clermont, C. Pontecorvo, L. Resnick, T. Zittoun, and B. Burge (New York, NY: Cambridge University Press), 3-10. doi: 10.1017/ cbo9780511616341.003

Piaget, J. (1928). The child's Conception of the World. London, UK: Routledge and Kegan Paul .

Piaget, J. (1952a). Play, Dreams and Imitation in Childhood. New York, NY: Norton. Piaget, J. (1952b). The origins of Intelligence in Children. New York, NY: Norton.

Piaget, J. (1954). The Construction of Reality in the Child. New York, NY: Basic Books.

Piaget, J. (1962). Play, Dreams and Imitation in Childhood. New York, NY: Norton.

Piaget, J. (1972). The Psychology of the Child. New York, NY: Basic Books.

Radford, L. (2003). On the epistemological limits of language: mathematical knowledge and social practice during the Renaissance. Educ. Stud. Math. 52, 123-150. 
Rodríguez, C., Moreno-Núñez, A., Basilio, M., and Sosa, N. (2015). Ostensive gestures come first: their role in the beginning of shared reference. Cogn. Dev. 36, 142-149. doi: 10.1016/j.cogdev.2015. 09.005 doi: 10.1016/j.cogdev.2015.09.005

Rodríguez, C., and Moro, C. (1998). El usoconvencionaltambiénhacepermanentes a los objetos. Infancia y Aprendizaje 21, 67-83.

Romanczyk, R. G., and Goren, E. R. (1975). Severe self-injurious behavior: the problem of clinical control. J. Consult. Clin. Psychol. 43, 730-739. doi: 10.1037/ 0022-006x.43.5.730

Savarese, G., Manzi, F., and Iannaccone, A. (2017). Social functions in ASD children and interaction with/through object: a brief report. Psychology 8, 1129-1133. doi: 10.4236/psych.2017.88073

Sigman, M., and Ungerer, J. A. (1984). Cognitive and language skills in autistic, mentally retarded, and normal children. Dev. Psychol. 20, 293-302. doi: 10. 1037/0012-1649.20.2.293

Tomasello, M. (2016). Cultural learning redux. Child Dev. 87, 643-653. doi: 10. $1111 /$ cdev.12499

Tomasello, M., Conti-Ramsden, G., and Ewert, B. (1990). Young children's conversations with their mothers and fathers: differences in breakdown and repair. J. Child Lang. 17, 115-130. doi: 10.1017/s0305000900013131

Trevarthen, C., and Hubley, P. (1978). "Secondary intersubjectivity: confidence, confiding, and acts of meaning in the first year," in Action, Gesture, and Symbol, ed. J. Lock (London: Academic Press), 183-229.

Turkle, S. (2004). Whither psychoanalysis in computer culture? Psychoanal. Psychol. 21, 16-30. doi: 10.1037/0736-9735.21.1.16
Vygotskij, L. S. (1976). Immaginazione e Creatività Infantile. Roma: Editori Riuniti. Vygotsky, L. S. (2004). Imagination and creativity in childhood. J. Russ. East Eur. Psychol. 42, 7-97.

Williams, E., Costall, A., and Reddy, V. (1999). Children with autism experience problems with both objects and people. J. Autism. Dev. Disord. 29, 367-378.

Williams, E., Kendell-Scott, L., and Costall, A. (2005). Parents' experiences of introducing everyday object use to their children with autism. Autism 9, 495-514. doi: 10.1177/13623613050 57869

Ziviani, J., Boyle, M., and Rodger, S. (2001). An introduction to play and the preschool child with autistic spectrum disorder. Br. J. Occup. Ther. 64, 17-22. doi: 10.1177/030802260106400104

Zucchermaglio, C. (1996). Vygotskij in Azienda. Apprendimento e Comunicazione nei Contesti Lavorativi. Roma: Carocci.

Conflict of Interest: The authors declare that the research was conducted in the absence of any commercial or financial relationships that could be construed as a potential conflict of interest.

Copyright (C) 2020 Manzi, Savarese, Mollo and Iannaccone. This is an open-access article distributed under the terms of the Creative Commons Attribution License (CC BY). The use, distribution or reproduction in other forums is permitted, provided the original author(s) and the copyright owner(s) are credited and that the original publication in this journal is cited, in accordance with accepted academic practice. No use, distribution or reproduction is permitted which does not comply with these terms. 


\section{APPENDIX}

\section{Appendix 1: Socio-Material Use of Objects (SMUO check-list)}

1. Is the child paying attention to the whole object, or to parts of it?

2. Is the child pointing at the object?

3. Has the child shared joint attention with the adult?

4. Does the child understand the use of the object?

5. For how many seconds does the child observe, indicate or touch the object?

6. Does the child imitate what the adult does with the object?

7. Is the child picking up the object as requested?

8. Does the child say the name of the object?

9. Does the child share the object with the adult?

10. Does the child use the object for its conventional purpose?

11. Does the child combine objects according to their conventional characteristics?

12. Does the child use the object to represent something else?

13. Does the child pretend to use an object that is present?

14. Does the child pretend to use an object that is not present? 


\section{OPEN ACCESS}

Edited by: Giulia Savarese,

University of Salerno, Italy

Reviewed by:

Sarina Hui-Lin Chien, China Medical University, Taiwan

Masahiro Hirai,

Nagoya University, Japan

*Correspondence: Kosuke Taniguch kosuket314@gmail.com

Specialty section: This article was submitted to

Developmental Psychology, a section of the journal Frontiers in Psychology

Received: 20 December 2019 Accepted: 20 July 2020

Published: 06 August 2020

Citation:

Taniguchi K, Tanabe-Ishibashi A and Itakura S (2020) The

Categorization of Objects With

Uniform Texture at Superordinate and Living/Non-living Levels in Infants:

An Exploratory Study.

Front. Psychol. 11:2009.

doi: 10.3389/fpsyg.2020.02009

\section{The Categorization of Objects With Uniform Texture at Superordinate and Living/Non-living Levels in Infants: An Exploratory Study}

\author{
Kosuke Taniguchi ${ }^{1 *}$, Azumi Tanabe-Ishibashi ${ }^{2}$ and Shoji Itakura ${ }^{1}$ \\ ${ }^{1}$ Center for Baby Science, Doshisha University, Kyoto, Japan, ${ }^{2}$ Institute of Development, Aging and Cancer, Tohoku \\ University, Miyagi, Japan
}

Human infants can categorize objects at various category levels (e.g., as a dog, animal, or living thing). It is crucial to understand how infants learn about the relationships between objects. This study investigated whether 4- to 11-month-old infants can categorize modeled objects at superordinate and living/non-living levels. In this experiment, we presented modeled objects with a uniform texture constructed by a $3 \mathrm{D}$ printer in animal, vegetable/fruit, vehicle, and tool categories and measured the time taken to examine novel categories. We investigated infants' categorization abilities using familiarization/novelty-preference tasks and their pre-linguistic development based on information from their parents. The analyses examined whether infants dedicated more examination time to objects in the new category at superordinate and living/non-living levels for each month of age. The results revealed that the examination time among 4- and 5-month-olds was at chance levels for both superordinate and living/non-living levels, while at 7 months, they showed high preference for the novel category at both category levels. For the superordinate level, the strength of response to living objects increased with linguistic development, while the strength of response to non-living objects did not depend on linguistic development. This indicates that the superordinate-level categorization of living objects depends on both perceptual information and linguistic ability. For the living/non-living level, the examination time for non-living objects increased with linguistic development. This implies that the recognition of non-living objects may depend on the development of object knowledge. The current study suggests that infants can recognize categories at an abstract level before the acquisition of linguistic representations while the category levels that infants can categorize objects are different for living/non-living objects. This may imply that infants learn the concepts of living/non-living via different mechanisms.

Keywords: object categorization, superordinate-level categories, living/non-living level categories, category hierarchy, infants

\section{INTRODUCTION}

We can, at a glance, recognize and categorize an object accurately and quickly (Thorpe et al., 1996). In fact, visual object recognition, which is largely associated with visual processing (Marr, 1982), constitutes a fundamental function of our daily lives. Moreover, one of the main goals of object recognition is object categorization, which depends on the abstraction levels of categories. 
Rosch et al. (1976) showed that objects at the basic level of the category (i.e., car) were categorized faster than at the superordinate (i.e., vehicle) and subordinate (i.e., Beetle) levels. Rosch et al. (1976) also found that children over 3 years of age were likely to learn object words at the basic level earlier than at other levels. They suggested that children learn object concepts at the basic level first, before subsequently acquiring the different category levels (i.e., the superordinate and subordinate levels).

However, some studies have revealed advantages of categorization at global levels over the basic level category for adults. Macé et al. (2009) showed that categorization at the superordinate level was faster than that at the basic level, using an ultra-rapid categorization task. They suggested that the visual system accesses object representation from a coarse/abstract level to a fine/specific level. The superordinate level categorization can access object representation from early visual information, and therefore permit faster decisions than for basic-level categorizations at short stimulus durations, although superordinate-level categorizations were slower at longer stimulus durations. This advantage of superordinate-level categorization is supported by Taniguchi et al. (2020), who showed that superordinate-level categorization does not depend on information representative of the category, but rather on perceptual information (e.g., complexity of shape). Greater accessibility of superordinate-level categorization has also been suggested by computational theory. Rogers and Patterson (2007) examined categorization performance at three category levels, namely basic, general (i.e., superordinate), and specific (i.e., subordinate), with four deadline conditions in a reaction-time assessment paradigm. They showed that behavior matched the predictions of parallel distributed processing (PDP) theory, indicating that semantic representation at the superordinate-level activates earlier than basic-level categorization, especially with shorter response deadlines (i.e., a requirement for faster responses). Thus, superordinate-level categorization is unlikely to depend on object representation, unlike basic and subordinate levels.

Mandler and McDonough (1993) investigated whether 7- to 11-month-old infants could categorize objects at the basic and global levels. The results showed that at 7 to 11 months, infants could categorize vehicle objects at the basic level (i.e., car from airplane and motorcycle), while they could not distinguish animal objects (i.e., dog from fish and rabbit). At the global level, infants could categorize animals and vehicles regardless of similarities among the categories such as shape and texture. Similarly, Behlchadha (1996) investigated the ability of 3- and 4-month-old infants in categorizing natural and artifactual objects, and the results indicated that they could categorize artifactual objects at both the basic and the global levels (i.e., furniture vs. animals). Likewise, Quinn and Johnson (2000) examined whether 2-month-olds can categorize objects at the basic (e.g., cat vs. elephant) and global (e.g., mammal vs. furniture) levels using a familiarization/novelty-preference task. Their results indicated that 2-month-olds could categorize at the global level, but not at the basic level. Therefore, infants may acquire global-level categorization earlier than basic-level categorization. This might be because infants' object categorization at the global level is based not only on perceptual information but on conceptual representation as well. Is global-level categorization influenced by linguistic development? To answer this question, in this study, we investigated whether the recognition of global-level categorization was influenced by linguistic development.

To understand the function of object categorization, it is essential to consider the relationship between the development of object categorization and that of linguistic ability. While some previous studies showed that presenting word labels facilitated categorization learning in infants (Balaban and Waxman, 1997; Fulkerson and Waxman, 2007). other studies indicated that infants' cognitive development acquires perceptual categorization, which helps acquisition of language accordingly (Mandler, 2004). Therefore, whether infants before the acquisition of language can categorize objects at global levels, such as superordinate and living/non-living levels, is a controversial point. To specify the relationship between linguistic development and object categorization at global levels, this study investigated infants' response to novel objects at superordinate and living/non-living levels.

Moreover, to understand the properties of the human visual system pertaining to object categorization, it is important to consider categorization at the living/non-living level. The ability to perceive and categorize an object as living is essential, such as when detecting a predator or finding food. Therefore, high sensitivity to living objects might be a characteristic of the human visual system (VanRullen and Thorpe, 2001). Moreover, when studying the category levels of an object, the superordinate-level category is usually defined as an animal, vegetable/fruit, vehicle, and furniture, according to the hierarchy of object category, whereas the more abstract level category can be defined as the living/non-living level. Studies on object categorization have often investigated the processing mechanisms of living/non-living categorization (e.g., Praß et al., 2013). For adults, categorization of items as living/non-living differed between living and non-living objects in behavioral and neuropsychological studies (McMullen and Purdy, 2006; Riddoch et al., 2008; Mahon et al., 2009). For the categorization of living objects, perceptual information is more crucial, whereas contextual and functional information is more critical for non-living objects (Garrard et al., 2001; Cree and McRae, 2003).

The sensitivity to living objects will be high even in infants, if categorizing an object as living is critical for survival. Pauen (2002) investigated whether 10- and 11-month-old infants could categorize living and non-living objects based on their perceived similarity by using an object-examination task with three-dimensional objects. The results indicated that infants were able to categorize living and non-living objects regardless of the similarity between the objects. Therefore, Pauen (2002) proposed that the categorization of living and non-living objects is based on knowledge-based processing. Some researchers have indicated that the structure of object concepts in infants and young children might differ from those of adults. For example, children aged between 3 and 5 years attribute life to non-living things, such as clouds and watches ("animistic;" Piaget, 1929). Herrmann et al. (2012) suggested that this error causes young children's lower accessibility to biological knowledge, although they already 
have it. Therefore, the acquisition of living/non-living concepts is predicted to relate with linguistic development than acquisition of the basic- and superordinate-level categories. However, Graham and Poulin-Dubois (1999) demonstrated that infants at the beginning of their linguistic development (approximately one and a half year-old) can learn categorical labels of objects by relying on shapes rather than textures (e.g., colors). They also showed that infants can categorize unknown objects as either animate or inanimate. Considering these findings, it is possible that infants learn categories based on shapes of objects before language acquisition (before 1-year-old). Furthermore, infants might be able to categorize objects at the living/non-living and superordinate levels based on their shapes. Thus, the question of how infants categorize at the living and non-living level is a highly controversial issue. Here, we examined these issues using $3 \mathrm{D}$ objects that had various shapes but a unitary texture.

Thus, to determine how the hierarchical structure of object categories is constructed in infants, we exploratively investigated whether infants' categorization ability is affected by linguistic development and whether objects are living vs. non-living, at both superordinate and living/non-living levels. Accordingly, we conducted familiarization/novelty-preference tasks using the object examination method among 4- to 11-month-olds, which is based on the logic by which infants' responses during the experiment should vary by the degree of perceptual differences among the stimuli (Pauen, 2002). We investigated differences in response strength by the category level of objects, by presenting a novel object in a different superordinate-level category or in the living/non-living category. Generally, the object examination method is believed to be appropriate for infants aged over 7 months (Mareschal and Quinn, 2001) but we applied this method for comparison with infants younger than 7 months. However, the object examination task used in this study was similar to that in a previous study that involved infants younger than 7 months; we used the familiarization/novelty-preference task (e.g., Quinn et al., 1993). In general, infants show more interest in 3D objects than in static images (Mandler, 2000; Perry, 2015). We, therefore, used the object examination method to examine whether infants can categorize objects at both the superordinate and living/non-living levels using perceptual information, such as that available from 3D shapes with a uniform texture. This study specifies the characteristics of global level categorization in infants, such as superordinate and living/non-living levels, assuming that infants' categorization is characterized by linguistic development. If infants' categorization at the global level depends only on perceptual information, then infants' preference for novel objects will not change with language development. Moreover, if the effects of linguistic development affect responsiveness to living objects, infants' preference for living/non-living objects will be different.

This study focused on the relation of shape information with category representation to investigate whether categorization at superordinate and living/non-living levels depends on linguistic development and whether objects are living/non-living. Shape information is the most important visual property in object recognition (Marr, 1982; Biederman, 1987; Ullman, 1996). This also applies to infants (Van de Walle et al., 1997).
3D printers can easily create objects with controlled shapes and textures. Therefore, in this study we assessed whether categorization processing from shape information among 4- to 11-month-olds differs between superordinate and living/non-living levels. This study used shape stimuli with a uniform texture produced by $3 \mathrm{D}$ printer.

\section{MATERIALS AND METHODS}

\section{Participants}

Thirty-four Japanese infants from 4 to 11 months old participated in this study (16 males and 18 females; $M=228.2$ days, $S D=66.92)$. Among them, we will investigate the effect of pre-linguistic development extensively. All participants were recruited from Doshisha University's waiting list. The design and purpose of the study were explained to the infants' parents and written informed consent documents were obtained from them before starting the experiment. The ethical community of Doshisha University approved of this research (approval number: 16091).

We calculated the sample size required to test the linear mixed model by using lmmpower function for longpower package in $R$, with 0.80 for beta (power of test) and 0.05 for alpha (significant level). The results indicated that 74 samples were needed. Approximately 19 participants were needed for this experiment, since four trials were repeated for each participant $(74 / 4=18.5)$.

\section{Stimuli}

The stimulus set was selected from animal, vegetable/fruit, vehicle, and tool categories which consisted of modeled objects of living (i.e., animal and vegetable/fruit; living object) and non-living (i.e., vehicle and tool; non-living object). A total of 16 modeled objects were presented in the experiment (animal: dog, lion, horse, and frog; vegetable/fruit: apple, strawberry, green pepper, and carrot; vehicle: car, bus, truck, and motor scooter; tool: hammer, saw, scoop, and broom; Figure 1). For animal categories, the objects were animals standing on their four legs, which represented complex shapes. The vegetable/fruit objects consisted of simple shape, such as rectangles or spheres. The vehicle objects involved rectangular shapes with large volume. The tool category consisted of objects that were essentially small-volume cylinders. The stimuli were constructed by a 3D printer (Stratasys, Objet30 Prime). Each stimulus size was set to approximately $8-15 \mathrm{~cm}$ on one side at maximum. The modeled objects were made of acrylic, translucent resin.

\section{Procedure}

Infants were seated on their parents' lap in front of the table. The experimenter was seated on the floor at the opposite side of table. The experimenter presented a stimulus (for familiarization blocks) or two stimuli (for test blocks) within the infants' reach. If an object fell off the table, the experimenter picked it up and put it back on the table as soon as possible. The task comprised four familiarization and four test blocks. These blocks were alternated (i.e., first familiarization block, then first test block, 


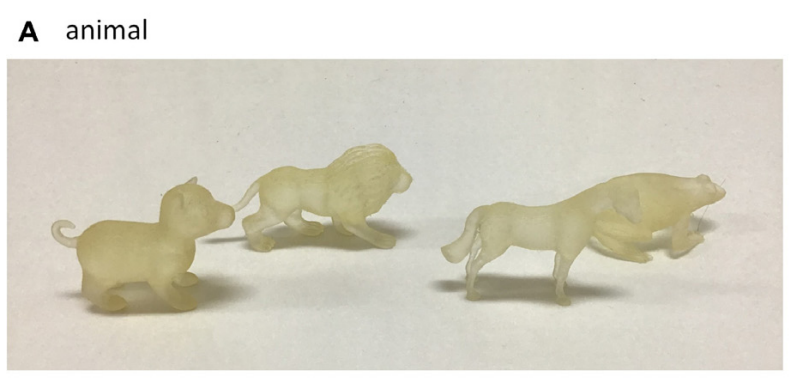

C vehicle

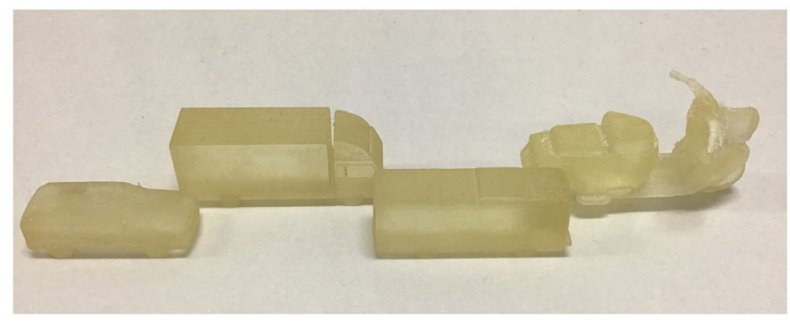

B vegetable/fruit

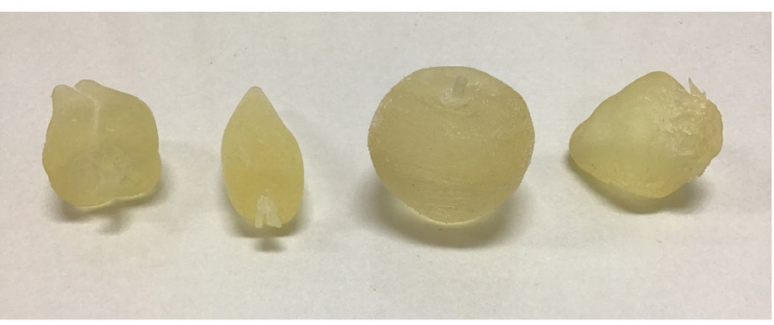

D tool

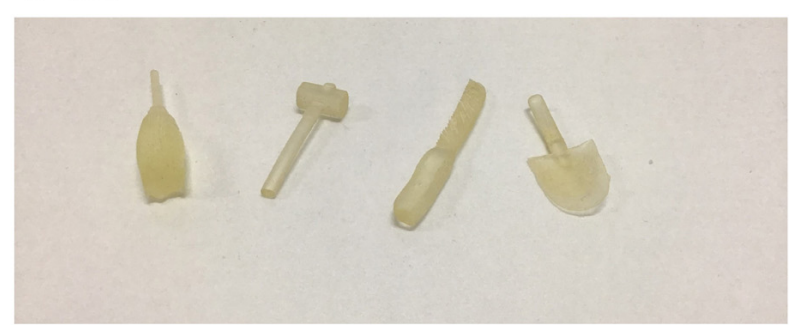

FIGURE 1 | The stimuli used in this experiment. The objects used in the animal (A),

, vegetable/fruit (B), vehicle (C), and tool (D) categories.

second familiarization block, ... fourth test block). When one block was finished, the next block was started immediately. The experiment was paused if the infant cried. Before starting the experiment, short warm-up trials were conducted by presenting blocks of various geometric shapes (i.e., cylinder, triangle pole, and square pole). The experimenter did not label any of the objects, and the parents were asked not to interact with the infants during the session. Completion of all blocks required approximately $30 \mathrm{~min}$.

For familiarization blocks, one of the three stimuli in a category was presented randomly. The infants could explore the object any way they wished (e.g., play, explore, and ignore). The stimulus was presented for a minimum of $20 \mathrm{~s}$, and if the infants showed interest in it after $20 \mathrm{~s}$, they could continue their examination until $180 \mathrm{~s}$. Then, the next stimulus was presented. Three stimuli in a category were presented once or twice. If infants did not respond to a new object, the familiarization block was terminated. In total, three to six trials were conducted in a familiarization block.

Test blocks were conducted immediately after a familiarization block was completed. For test blocks, two stimuli were presented simultaneously beside each other: one was the novel object in the category presented in the familiarization block and the other was an object from a novel category. We randomly assigned the positions of the two stimuli. The novel category was chosen based on superordinate (e.g., animal vs. vegetable/fruit and vehicle vs. tool) or living/non-living levels (e.g., animal vs. vehicle and vegetable/fruit vs. tool). The infants could explore the objects the same way as in the familiarization blocks. A test block consisted of one trial. After the trial was finished, the next familiarization block was started.

The behavior of infants in the experiment was recorded using a video camera placed in front of them. After four test blocks were completed, the experimenter orally asked infants' parents questions on the pre-linguistic scale and recoded their answers.

\section{Pre-linguistic Scales}

To investigate the effects of linguistic development, we asked parents for their responses to the Nagao Pre-Linguistic Scales (Nagao et al., 1990). The scales were used in 12 questions selected from the stage of language development and representing the function of language at 1-12 months, which involved development of vocal expressions and symbolistic functions. Vocal expressions also involved infants' communication ability through aspects such as emotion expression and attracting mother's attention (but not by crying). Nagao et al. (1990) argued that these communication abilities indicate the differentiation of objects and are associated with the development of conceptual function. The score on the pre-linguistic scales, therefore, indicated the degree of development in conceptual function.

The score rating was followed by Nagao et al. (1990): If it certainly acknowledged, the score was 1 . If it had started appearance but it does not completely, the score was 0.5 . If it had not appeared yet, the score was 0 .

\section{Video Coding}

One coder for the familiarization blocks and two coders for test blocks measured the examination time for each trial. They were not aware of the purpose of this study. For familiarization blocks, the coder recorded the examination time, defined as the time during which an infant examines an object by using observation and touch. Examination time was measured from the beginning of the stimulus presentation until the infant stopped examining the stimulus. For test blocks, the coders recorded the examination time of each stimulus over $20 \mathrm{~s}$ from the time of first response to a 
stimulus, such as the first look or touch. The examination time may indicate the strength of response to the stimulus (Pauen, 2002).

\section{Statistical Analysis Methods}

We conducted repeated-measures linear mixed-models using the lme4 (Bates et al., 2015) and lmerTest (Kuznetsova et al., 2017). Packages in $\mathrm{R}$ to compare the difference between group means in repeated measure and mixed design. This procedure produces results similar to those of ANOVA and $t$-tests. In a linear mixed-model, the random participant effect is constructed using the correlations between the observations of the participants. Linear mixed-models are generally robust against missing values (Baayen et al., 2008).

\section{RESULTS}

Data from two female infants (an 8-month-old and an 11-month-old) were excluded from the analyses, because they did not show any response to the stimuli. The mean examination time in trials between coders for test blocks provided the raw data for further analyses because intercoder reliability was high $(r=0.94$, range: $0.93-0.96$ ).

\section{Familiarization Trials}

To examine whether infants were habituated to the presented stimulus or category, the model set examination time as the dependent variable, trials in a block (centered at first trial: First trials) as the continuous variable, days-old [centered at the mean (227.45 days) of days-old: days-old] as the fixed variable, and infant ID as the random variable. The analyses involved 475 observations from 32 participants. The analysis revealed a significant effect of First trials and Days-old [First trials: beta $=-12.53, t(453)=-6.67, p<0.001$; Days-old: beta $=0.46, t(44)=4.73, p<0.001]$ and the interaction between them [beta $=-0.09, t(455)=-3.08, p<0.01$ ], indicating that examination time decreased with trials number (Figure 2 and Table 1).

\section{Test Trials}

The analyses involved 119 observations from 32 participants. As preliminary analysis, we conducted a hierarchical multiple regression analysis of the linear mixed model in order to investigate whether there are differences in infant preferences by category of novel object. In the model, preference scores were used as the dependent variable, while the category of novel object was used as a fixed variable and the infant's ID was used as a random variable. The results showed that the main effect of the category was not significant $\left[\chi^{2}(3)=5.30\right.$, n.s. $]$.

The analysis focused on the intercept because we examined whether or not the preference score was more than the chance-level (i.e., 0.5). We set the months-old variable as dummy variable centered at each month, to investigate effects of age change. The series of analyses revealed that the effect of the intercept was significant in 7-month-olds at both superordinate

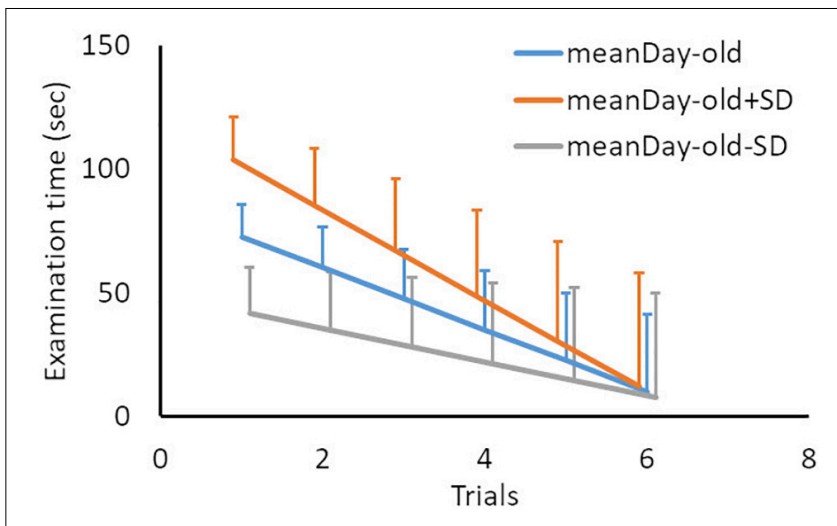

FIGURE 2 | Mean examination time and the prediction from the linear mixed-model in the familiarization test, as a function of familiarization trial. "meanDay-old" indicates the mean age of participants in days (221.57), while "meanDay-old $\pm S D$ " denotes the mean age $\pm 1 S D$ (67.15), to show the interaction effect between age in days and trial number. Error bars indicate standard errors.

TABLE 1 | The estimations of the linear mixed-model based on the familiarization trials.

\begin{tabular}{lrrrrr}
\hline & Beta & SE & $\boldsymbol{d f}$ & $\boldsymbol{t}$-value & $\boldsymbol{p}$ \\
\hline Intercept & 72.54 & 6.60 & 45.14 & 11.00 & $<0.001$ \\
Days-old & 0.46 & 0.10 & 44.75 & 4.73 & $<0.001$ \\
First trials & -12.53 & 1.88 & 453.79 & -6.67 & $<0.001$ \\
Days-old $\times$ first trials & -0.09 & 0.03 & 455.90 & -3.08 & 0.002 \\
\hline
\end{tabular}

and living/non-living levels [beta $>0.22, t(110)>2.58, p<0.05$ ] The intercept effect in 10-month-olds was significant for the superordinate-level [beta $=0.25, t(110)=2.00, p<0.05$ ], while the intercept effect approached significance in 10-month-olds for the living/non-living level [beta $=0.23, t(110)=1.88, p<0.1$ ] The intercept effect was not significant in infants of other ages. In the contrast, the effect of category level was not significant in infants of any age (Figure 3 ).

To test the difference between the categorization of superordinate and living/non-living levels by linguistic development and object properties in the living/non-living category for a new category, a series of a linear mixed modeling effects was conducted. The model set the preference score (centered at 0.5 ) as the dependent variable. Pre-linguistic score (centered at mean: linguistics) was set as the continuous variable, categorization level (superordinate vs. living/non-living level: category level) was set as the fixed variable, living/non-living category of objects (living object vs. non-living object: object category) were set as dummy variables, and the interactions among them were also included in the model. Infant ID was set as a random effect. The analyses involved 119 observations from 32 participants. Note that the effect of age in days-old was omitted from the fixed variables in these analyses, to avoid multi-collinearity with pre-linguistic score $[r(117)=0.67$, $p<0.001]^{1}$. The results revealed significant two-way interactions

${ }^{1}$ We did not analyze the data by dividing the infants into younger and older categories because we aimed to investigate the effects of pre-linguistic development 


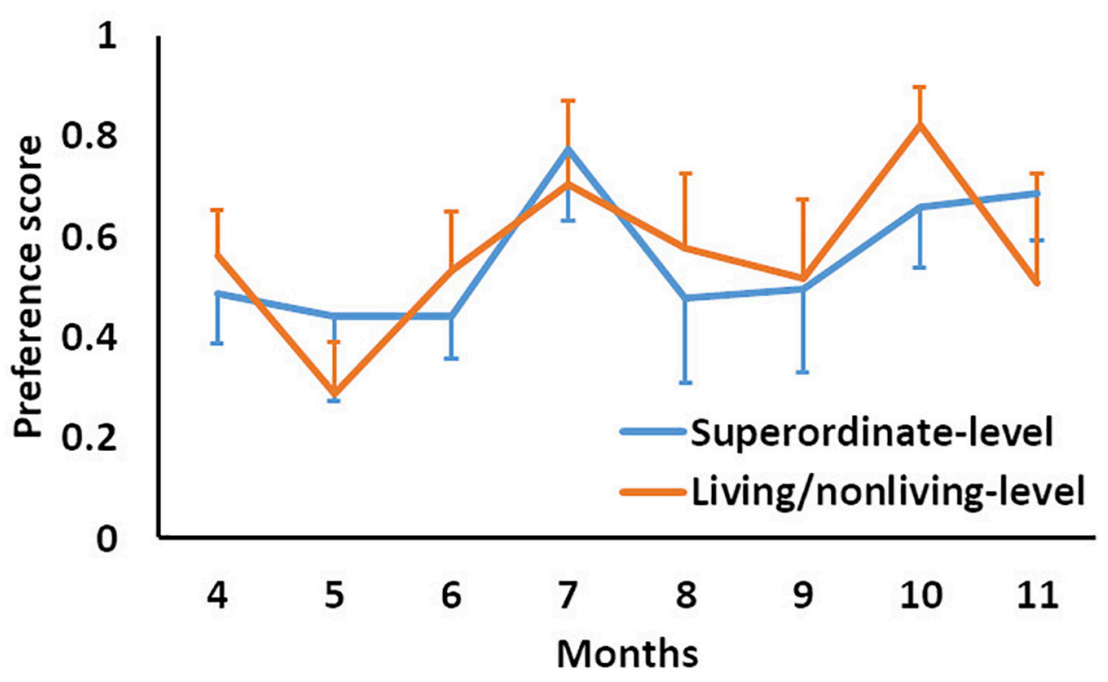

FIGURE 3 | Mean preference score as a function of months and category level. Error bars indicate standard errors.

between pre-linguistic score and category level [beta $=0.12$, $t(111)=2.77, p<0.01]$ and between pre-linguistic score and object category [beta $=0.09, t(111)=2.09, p<0.05]$. The three-way interaction among pre-linguistic score, category level, and object category was also significant [beta $=-0.23$, $t(111)=-3.76, p<0.001$; as shown in Table 2]. Figure 4 shows the results of analyses of simple main effects of the three-way interaction, showing that preference scores for living objects at the superordinate level were higher than those at the living/non-living level for infants with higher linguistic ability. In addition, preference scores for living objects were higher than those for non-living objects at the superordinate level of categorization for infants with higher linguistic ability. As Figure 4 shows, the linguistic effects were revealed at the superordinate-level categorization of living objects and at the living/non-living level of non-living objects.

exploratively. Moreover, we also conducted the repeated measure mixed model analysis by using a similar procedure in the analysis of test trials, except that the age group [younger than 7 months (i.e., 4- to 6-month-olds) vs. 7 months or older (i.e., 7- to 11-month-olds)] rather than pre-linguistic score was set as a dummy dependent variable. The findings indicated results almost identical to those indicated in Figure $\mathbf{3}$ and Table $\mathbf{2}$ (see Supplementary Figure $\mathbf{1}$ and Supplementary Table 1).

\section{DISCUSSION}

In this study, we investigated whether categorization at both the superordinate and living/non-living levels depends on linguistic development and whether objects are living/non-living in 4- to 11-month-old infants. The results of this study indicated that infants older than 7 months could categorize objects at both the superordinate and living/non-living levels. However, the strength of response was associated with an interaction between living/non-living objects and category levels. Infants with higher pre-linguistic scores showed higher preference scores for living objects at the superordinate level than at the living/non-living level of categorization. Infants at 7 months could distinguish the category at both the superordinate and living/non-living levels, although their processing was similar. The processing of superordinate and living/non-living levels may differ owing to linguistic development.

For superordinate-level categorization, the effects of linguistic development differed between living and non-living objects: the linguistic score did not influence preference scores for non-living objects, although it did increase the examination time for living objects. This might indicate that superordinate-level categorization depends on both perceptual information

TABLE 2 | The estimations of the linear mixed-model based on the test trials.

\begin{tabular}{|c|c|c|c|c|c|}
\hline & Beta & $S E$ & $d f$ & $t$-value & $p$ \\
\hline Intercept & 0.08 & 0.06 & 111.00 & 1.32 & 0.191 \\
\hline Pre-linguistic score & -0.03 & 0.03 & 111.00 & -1.01 & 0.317 \\
\hline Superordinate-level & 0.07 & 0.09 & 111.00 & 0.80 & 0.424 \\
\hline Non-living object & -0.05 & 0.09 & 111.00 & -0.55 & 0.585 \\
\hline Pre-linguistic score $\times$ superordinate level & 0.12 & 0.04 & 111.00 & 2.77 & 0.007 \\
\hline Pre-linguistic score $\times$ non-living object & 0.09 & 0.04 & 111.00 & 2.09 & 0.039 \\
\hline Superordinate level $\times$ non-living object & -0.11 & 0.12 & 111.00 & -0.92 & 0.359 \\
\hline Pre-linguistic score $\times$ superordinate level $\times$ non-living object & -0.23 & 0.06 & 111.00 & -3.76 & $<0.001$ \\
\hline
\end{tabular}




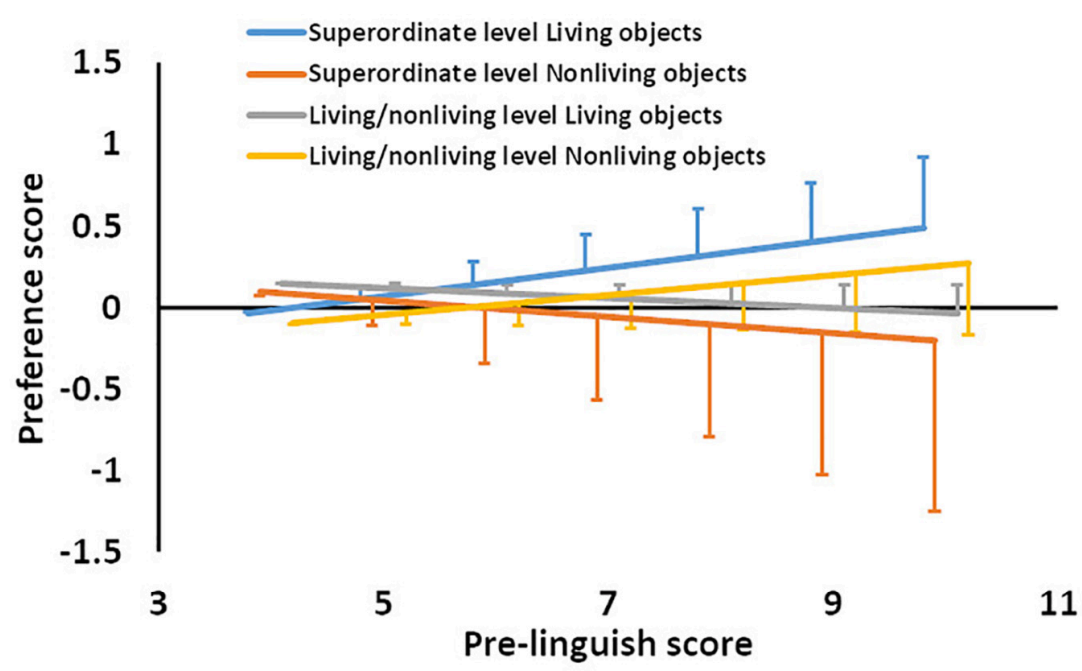

FIGURE 4 | The prediction from the linear mixed-model as a function of pre-linguistic score, category level, and living/non-living object. Error bars indicate standard errors.

and linguistic ability for living objects. These results are consistent with a previous study that showed both pre-existing representation and perceptual information related to infants' categorization abilities (Smith, 2000; Pauen, 2002). Therefore, infants categorize living objects at the superordinate-level depending not only on perceptual information but also on object concepts.

For non-living objects at the superordinate-level categorization, no effect of linguistic ability was found. This might be because perceptual shape information plays an important role in the categorization of non-living objects. Some studies found that 2-year-old children categorize non-living objects based on functional features (e.g., Deborah et al., 2004; Casler and Kelemen, 2007), which indicates that functional features are important for shaping the concepts of non-living objects. The current study also indicated that 7-month-olds can categorize objects at both superordinate levels. Thus, it might be possible for infants to perceive functional features from the shape of objects at circa 7 months of age.

For categorization at living/non-living level, preference scores for non-living objects increased correspondingly to pre-linguistic scores, while those for living objects were unaffected by pre-linguistic scores. These results suggest that perceptual information is more effective for categorization of living objects at the living/non-living level, because the shape components of living objects were more complex than those of non-living objects. That is, the living category consisted of rounded and curved surfaces, while the non-living category consisted of straight lines, right angles, and corners. However, the response strength did not different according to linguistic development for non-living objects. Infants generally prefer more complex, informative objects over other types of objects (e.g., Fantz, 1958). Thus, response strength is expected to be greater for living objects. Moreover, 10-month-olds cannot categorize objects at the living/non-living level but can at the superordinate level.
Therefore, more developed object knowledge than that exhibited by 11-month-olds might be necessary for infants to categorize at the living/non-living level.

In this study, we used familiarization/novel-preference tasks with $3 \mathrm{D}$ objects to measure the strength of category preference by considering both fixation and the touching of objects (Oakes et al., 1991; Oakes and Tellinghuisen, 1994). The results indicated that the 4- and 5-month-old infants did not show any preference for the novel category, which was inconsistent with Quinn and Johnson (2000). Mareschal and Quinn (2001) argued that young infants form an exclusive category when the examined objects have low variability in perceptual features. As we used objects with a uniform texture in the current study, young infants might not have formed inclusive categories of several objects. Moreover, the familiarization trials showed that 4- and 5-month-olds also did not show habituation to objects, because they showed weak responses in both familiarization and test trials in this study. Therefore, for 4- and 5-month-old infants, shape information might not be sufficient for categorization.

In the current study, the recognition of living objects was found at the living/non-living level of categorization. In some studies, recognition of living objects was not found. For example, VanRullen and Thorpe (2001) investigated whether living objects (i.e., mammals) were categorized faster than non-living objects (i.e., means of transport), using ultra-rapid categorization tasks. They revealed that response speed for living objects was not greater than that for non-living objects. This might have been due to a category-level effect as, in the current study, we found different effects of living objects between superordinate and living/non-living levels. Further studies will be needed to clarify the living objects effect.

Additional issues of categorization at a global level relate to the variety of objects within a category. For example, the shapes of birds and fishes are very different from the shapes of the animals used in this study. In this study, the stimuli 
of objects were limited, and infants were familiarized with a single category in a familiarization block. Therefore, the question remains as to how infants categorize these different shape objects within the same category. However, the current study indicated that categorization by infants might depend not only on shape information, but also on linguistic development of objects. These findings provide insight into the how the hierarchical structure of object concepts is acquired.

Recognizing objects is a critical life skill (e.g., for finding food, grasping a bottle, or communicating with others). Some diseases could cause deficits in object recognition ability. For example, patients with semantic dementia show gradually increasing deficits in conceptual knowledge regarding word and object meanings. Such patients sometimes show impaired categorization at basic and subordinate levels, but intact categorization at the superordinate level (Rogers and Patterson, 2007). Furthermore, individuals with autism spectrum disorder (ASD) show difficulties in recognizing atypical objects (Gastgeb and Strauss, 2012). This may lead to problems for individuals with ASD in solving problems that involve abstract content (Alderson-Day and McGonigle-Chalmers, 2011). According to these studies, the deterioration of object recognition may cause difficulties with daily living. The current study implies that the learning of object concepts may differ between living and non-living objects, given the different response strength at the category level. Difficulties in daily living might be caused by distortion of the structure of object concepts. Thus, the current study contributes to extant work by revealing correlations between object recognition ability and other abilities, such as grasping a bottle, pointing an object, and communicating with others.

To summarize, this study investigated how 4 - to 11-month-old infants categorized objects at the superordinate and living/non-living levels, using $3 \mathrm{D}$ objects with a uniform texture. We conducted object examination trials and measured the duration of infants' examination of the stimuli. The results showed that preference of a novel category was influenced by whether objects were living/non-living and linguistic development. For the superordinate-level category, the strength of response to living objects increased with linguistic development, but that to non-living objects was unaffected by linguistic development. This indicates that the superordinate-level categorization of living objects depends on both perceptual information and linguistic ability, consistent with the idea of superordinate-level recognition. For the living/non-living category, preference scores did not differ between living and non-living objects. This implies that

\section{REFERENCES}

Alderson-Day, B., and McGonigle-Chalmers, M. (2011). Is it a bird? Is it a plane? Category use in problem-solving in children with autism spectrum disorders. J. Autism Dev. Disord. 41, 555-565. doi: 10.1007/s10803-010-1077-9

Baayen, R. H., Davidson, D. J., and Bates, D. M. (2008). Mixed-effects modeling with crossed random effects for subjects and items. J. Mem. Lang. 59, 390-412. doi: 10.1016/J.JML.2007.12.005 categorization at the living/non-living level may depend on more developed object knowledge and finer perceptual discrimination. The current study suggests that infants can recognize categories at an abstract level, using not only shape information of objects, but also linguistic representations.

\section{DATA AVAILABILITY STATEMENT}

The datasets generated for this study are available on request to the corresponding author.

\section{ETHICS STATEMENT}

The studies involving human participants were reviewed and approved by the ethical community of Doshisha University. Written informed consent to participate in this study was provided by the participants' legal guardian/next of kin.

\section{AUTHOR CONTRIBUTIONS}

KT and AT-I designed this study and wrote the original draft. KT conducted the experiments and analyzed the results. SI supervised this project. All authors contributed to revisions, read, and approved the submit version.

\section{FUNDING}

This study was supported by MEXT Promotion of Distinctive Joint Research Center Program Grant Number JPMXP0619217850.

\section{ACKNOWLEDGMENTS}

We are grateful to Chikako Hashizume and Rieko Higashitani for their assistance with video coding. We also thank the Keihanna Research-Complex project for supporting 3D printer usage.

\section{SUPPLEMENTARY MATERIAL}

The Supplementary Material for this article can be found online at: https://www.frontiersin.org/articles/10.3389/fpsyg. 2020.02009/full\#supplementary-material

Balaban, M. T., and Waxman, S. R. (1997). Do Words Facilitate Object Categorization in 9-Month-Old Infants? J. Exp. Child Psychol. 64, 3-26. doi: 10.1006/JECP.1996.2332

Bates, D., Mächler, M., Bolker, B., and Walker, S. (2015). Fitting Linear MixedEffects Models Using lme4. J. Stat. Softw. 1,

Behl-chadha, G. (1996). Basic-level and superordinate-like categorical representations in early infancy. Cognition 60, 105-141. doi: 10.1016/ 0010-0277(96)00706-8 
Biederman, I. (1987). Recognition-by-components: a theory of human image understanding. Psychol. Rev. 94, 115-147. doi: 10.1037/0033-295X.94. 2.115

Casler, K., and Kelemen, D. (2007). Reasoning about artifacts at 24 months: the developing teleo-functional stance. Cognition 103, 120-130. doi: 10.1016/ j.cognition.2006.02.006

Cree, G. S., and McRae, K. (2003). Analyzing the factors underlying the structure and computation of the meaning of chipmunk, cherry, chisel, cheese, and cello (and many other such concrete nouns). J. Exp. Psychol. Gen. 132, 163-201. doi: 10.1037/0096-3445.132.2.163

Deborah, G. K. N., Louisa Chan, E., and Holt, M. B. (2004). When Children Ask, "What Is It?". What Do They Want to Know About Artifacts? Psychol. Sci. 15, 384-389. doi: 10.1111/j.0956-7976.2004.00689.x

Fantz, R. L. (1958). Pattern vision in young infants. Psychol. Rec. 8, 43-47. doi: $10.1007 / \mathrm{bf03393306}$

Fulkerson, A. L., and Waxman, S. R. (2007). Words (but not Tones) facilitate object categorization: evidence from 6- and 12-month-olds. Cognition 105, 218-228. doi: 10.1016/J.COGNITION.2006.09.005

Garrard, P., Lambon Ralph, M. A., Hodges, J. R., and Patterson, K. (2001). Prototypicality, distinctiveness, and intercorrelation: analyses of the semantic attributes of living and nonliving concepts. Cogn. Neuropsychol. 18, 125-174. doi: 10.1080/02643290125857

Gastgeb, H. Z., and Strauss, M. S. (2012). Categorization in ASD: the role of typicality and development. Perspect. Lang. Learn. Educ. 19, 66-74. doi: 10. 1044/lle19.2.66

Graham, S. A., and Poulin-Dubois, D. (1999). Infants' reliance on shape to generalize novel labels to animate and inanimate objects. J. Child Lang. 26, 295-320. doi: 10.1017/S0305000999003815

Herrmann, P. A., Medin, D. L., and Waxman, S. R. (2012). When humans become animals: development of the animal category in early childhood. Cognition 122, 74-79. doi: 10.1016/j.cognition.2011.08.011

Kuznetsova, A., Brockhoff, P. B., and Christensen, R. H. B. (2017). ImerTest Package: tests in Linear Mixed Effects Models. J. Stat. Softw. 1, 1-26.

Macé, M. J.-M., Joubert, O. R., Nespoulous, J.-L., and Fabre-Thorpe, M. (2009). The time-course of visual categorizations: you spot the animal faster than the bird. PLoS One 4:e5927. doi: 10.1371/journal.pone.0005927

Mahon, B. Z., Anzellotti, S., Schwarzbach, J., Zampini, M., and Caramazza, A. (2009). Category-specific organization in the human brain does not require visual experience. Neuron 63, 397-405. doi: 10.1016/j.neuron.2009. 07.012

Mandler, J. M. (2000). Perceptual and Conceptual Processes in Infancy. J. Cogn. Dev. 1, 3-36. doi: 10.1207/S15327647JCD0101N_2

Mandler, J. M. (2004). Thought before language. Trends Cogn. Sci. 8, 508-513. doi: 10.1016/j.tics.2004.09.004

Mandler, J. M., and McDonough, L. (1993). Concept formation in infancy. Cogn. Dev. 8, 291-318. doi: 10.1016/S0885-2014(93)80003-C

Mareschal, D., and Quinn, P. C. (2001). Categorization in infancy. Trends Cogn. Sci. 5, 443-450. doi: 10.1016/S1364-6613(00)01752-6

Marr, D. (1982). Vision: A Computational Investigation into the Human Representation and Processing of Visual Information. San Francisco, CA: W. H. Freeman.

McMullen, P. A., and Purdy, K. S. (2006). Category-specific effects on the identification of non-manipulable objects. Brain Cogn. 62, 228-240. doi: 10. 1016/j.bandc.2006.06.002

Nagao, K., Shino, K., and Ueyoshi, A. (1990). Test method for pre-speech language: I. Outline of test. Hattatsu 22, 319-326. doi: 10.11251/ojjscn1969. 22.319
Oakes, L. M., Madole, K. L., and Cohen, L. B. (1991). Infants' object examining: habituation and categorization. Cogn. Dev. 6, 377-392. doi: 10.1016/08852014(91)90045-F

Oakes, L. M., and Tellinghuisen, D. J. (1994). Examining in infancy: does it reflect active processing? Dev. Psychol. 30, 748-756. doi: 10.1037/0012-1649.30.5.748

Pauen, S. (2002). Evidence for knowledge-based category discrimination in infancy. Child. Dev. 73, 1016-1033. doi: 10.1111/1467-8624.00454

Perry, L. K. (2015). To have and to hold: looking vs. touching in the study of categorization. Front. Psychol. 6:178. doi: 10.3389/fpsyg.2015.00178

Piaget, J. (1929). The Child's Conception of the World (J. Tomlinson \& A. Tomlinson, Trans.). London: Kegan Paul, Trench, Trubner \& Co. Ltd.

Praß, M., Grimsen, C., König, M., and Fahle, M. (2013). Ultra rapid object categorization: effects of level, animacy and context. PLoS One 8:e68051. doi: 10.1371/journal.pone.0068051

Quinn, P. C., Eimas, P. D., and Rosenkrantz, S. L. (1993). Evidence for Representations of Perceptually Similar Natural Categories by 3-Month-Old and 4-Month-Old Infants. Perception 22, 463-475. doi: 10.1068/p220463

Quinn, P. C., and Johnson, M. H. (2000). Global-before-basic object categorization in connectionist networks and 2-month-old infants. Infancy 1, 31-46. doi: 10.1207/S15327078IN0101_04

Riddoch, M. J., Humphreys, G. W., Akhtar, N., Allen, H., Bracewell, R. M., and Schofield, A. J. (2008). A tale of two agnosias: distinctions between form and integrative agnosia. Cogn. Neuropsychol. 25, 56-92. doi: 10.1080/ 02643290701848901

Rogers, T. T., and Patterson, K. (2007). Object categorization: reversals and explanations of the basic-level advantage. J. Exp. Psychol. Gen. 136, 451-469. doi: 10.1037/0096-3445.136.3.451

Rosch, E., Mervis, C. B., Gray, W. D., and Boyes-Braem, P. (1976). Basic objects in natural categories. Cogn. Psychol. 8, 382-439. doi: 10.1016/0010-0285(76) 90013-x

Smith, L. B. (2000). From knowledge to knowing: real progress in the study of infant categorization. Infancy 1, 91-97. doi: 10.1207/S15327078IN0101_08

Taniguchi, K., Kuraguchi, K., Takano, Y., and Itakura, S. (2020). Object categorization processing differs according to category level: comparing visual information between the basic and superordinate levels. Front. Psychol. 11:501. doi: 10.3389/fpsyg.2020.00501

Thorpe, S., Fize, D., and Marlot, C. (1996). Speed of processing in the human visual system. Nature 381, 520-522. doi: 10.1038/381520a0

Ullman, S. (1996). High-level Vision: Object Recognition and Visual Cognition. Cambridge, MA: MIT Press.

Van de Walle, G., Spelke, E. S., and Carey, S. (1997). "Concepts and categorization in infancy," in Proceedings of the Biennial Meeting of the Society for Research in Child Development, Washington, DC.

VanRullen, R., and Thorpe, S. J. (2001). Is it a bird? Is it a plane? Ultra-rapid visual categorisation of natural and artifactual objects. Perception 30, 655-668. doi: $10.1068 /$ p3029

Conflict of Interest: The authors declare that the research was conducted in the absence of any commercial or financial relationships that could be construed as a potential conflict of interest.

Copyright (c) 2020 Taniguchi, Tanabe-Ishibashi and Itakura. This is an open-access article distributed under the terms of the Creative Commons Attribution License (CC BY). The use, distribution or reproduction in other forums is permitted, provided the original author(s) and the copyright owner(s) are credited and that the original publication in this journal is cited, in accordance with accepted academic practice. No use, distribution or reproduction is permitted which does not comply with these terms. 


\section{OPEN ACCESS}

Edited by:

Emily Mather,

University of Hull, United Kingdom

Reviewed by:

Muneeb Imtiaz Ahmad,

Edinburgh Centre for Robotics,

Heriot-Watt University,

United Kingdom

Sergio Morra,

University of Genoa, Italy

*Correspondence:

Federico Manzi

federico.manzi@unicatt.it

tThese authors have contributed equally to this work

Specialty section:

This article was submitted to Developmental Psychology,

a section of the journal

Frontiers in Psychology

Received: 20 December 2019

Accepted: 20 July 2020

Published: 30 September 2020

Citation:

Manzi F, Peretti G, Di Dio C, Cangelosi A, Itakura S, Kanda T,

Ishiguro $H$, Massaro $D$ and

Marchetti A (2020) A Robot Is Not Worth Another: Exploring Children's Mental State Attribution to Different

Humanoid Robots.

Front. Psychol. 11:2011. doi: 10.3389/fpsyg.2020.02011

\section{A Robot Is Not Worth Another: Exploring Children's Mental State Attribution to Different Humanoid Robots}

\author{
Federico Manzi ${ }^{1 *}$, Giulia Peretti1t, Cinzia Di Dio'1, Angelo Cangelosi², Shoji Itakura ${ }^{3}$, \\ Takayuki Kanda ${ }^{4,5}$, Hiroshi Ishiguro ${ }^{5,6}$, Davide Massaro ${ }^{1}$ and Antonella Marchetti ${ }^{1}$
}

${ }^{1}$ Research Unit on Theory of Mind, Department of Psychology, Università Cattolica del Sacro Cuore, Milan, Italy, ${ }^{2}$ School of Computer Science, University of Manchester, Manchester, United Kingdom, ${ }^{3}$ Centre for Baby Science, Doshisha University, Kyoto, Japan, ${ }^{4}$ Human-Robot Interaction Laboratory, Graduate School of Informatics, Kyoto University, Kyoto, Japan, ${ }^{5}$ Advanced Telecommunications Research Institute International, IRC/HIL, Keihanna Science City, Kyoto, Japan,

${ }^{6}$ Department of Systems Innovation, Osaka University, Toyonaka, Japan

Recent technological developments in robotics has driven the design and production of different humanoid robots. Several studies have highlighted that the presence of humanlike physical features could lead both adults and children to anthropomorphize the robots. In the present study we aimed to compare the attribution of mental states to two humanoid robots, NAO and Robovie, which differed in the degree of anthropomorphism. Children aged 5, 7, and 9 years were required to attribute mental states to the NAO robot, which presents more human-like characteristics compared to the Robovie robot, whose physical features look more mechanical. The results on mental state attribution as a function of children's age and robot type showed that 5-year-olds have a greater tendency to anthropomorphize robots than older children, regardless of the type of robot. Moreover, the findings revealed that, although children aged 7 and 9 years attributed a certain degree of human-like mental features to both robots, they attributed greater mental states to NAO than Robovie compared to younger children. These results generally show that children tend to anthropomorphize humanoid robots that also present some mechanical characteristics, such as Robovie. Nevertheless, age-related differences showed that they should be endowed with physical characteristics closely resembling human ones to increase older children's perception of human likeness. These findings have important implications for the design of robots, which also needs to consider the user's target age, as well as for the generalizability issue of research findings that are commonly associated with the use of specific types of robots.

Keywords: child-robot interaction (cHRI), social robots, humanoid and anthropomorphic robots, differences among robots, children, anthropomorphism, mental states attribution

\section{INTRODUCTION}

Currently, we are witnessing an increasing deployment of social robots (Bartneck and Forlizzi, 2004) in various contexts, from occupational to clinical to educational (Murashov et al., 2016; Belpaeme et al., 2018; Marchetti et al., in press). Humanoid social robots (HSRs), in particular, have proven to be effective social partners, possibly due to their physical human likeness 
(Dario et al., 2001). Humanoid social robots can vary in the degree of their anthropomorphic physical characteristics, often depending on the target user (children, adults, elderly, students, clinical populations, etc.) and the context (household, education, commercial, and rehabilitation). For example, the humanoid KASPAR robot that resembles a young child (with face, arms and hands, legs and feet), was specifically built for children with autism spectrum disorder (Dautenhahn et al., 2009; Wainer et al., 2014). In other instances, however, the same HSRs are used both for different purposes and different populations, like the NAO robot, which is largely used both with clinical and non-clinical populations (Shamsuddin et al., 2012; Mubin et al., 2013; Begum et al., 2016; Belpaeme et al., 2018), or the Robovie robot, that is employed both with adults and children (Shiomi et al., 2006; Kahn et al., 2012). A recent review of the literature by Marchetti et al. (2018) showed that different physical characteristics of HSRs may significantly affect the quality of interaction between humans and robots at different ages. The construction of robots that integrate and expand the specific biological abilities of our species led to two different directions in robotic development based on different, though related, theoretical perspectives: developmental cybernetics (DC; Itakura, 2008; Itakura et al., 2008; Moriguchi et al., 2011; Kannegiesser et al., 2015; Okanda et al., 2018; Di Dio et al., 2019; Wang et al., 2020; Manzi et al., 2020a) and developmental robotics (DR; De La Cruz et al., 2014; Cangelosi and Schlesinger, 2015, 2018; Lyon et al., 2016; Morse and Cangelosi, 2017; Vinanzi et al., 2019; Zhong et al., 2019; Di Dio et al., 2020a,b). The first perspective (DC) consists of creating a human-like system, by simulating human psychological processes and prosthetic functions in the robot (enhancing the function and lifestyle of persons) to observe people's behavioral response toward the robot. The second perspective (DR) is related to the development of cognitive neural networks in the robot that would allow it to autonomously gain sensorimotor and mental capabilities with growing complexity, starting from intricate evolutionary principles. From these premises, the next two paragraphs briefly outline current findings concerning the effect that physical features of the HSRs have on human perception, thus outlining the phenomenon of anthropomorphism, and a recent methodology devised to measure it.

\section{Anthropomorphism}

Anthropomorphism is a widely observed phenomenon in human-robot interaction (HRI; Fink et al., 2012; Airenti, 2015; Złotowski et al., 2015), and it is also greatly considered in the design of robots (Dario et al., 2001; Kiesler et al., 2008; Bartneck et al., 2009; Sharkey and Sharkey, 2011; Zanatto et al., 2016, 2020). In psychological terms, anthropomorphism is the tendency to attribute human characteristics, physical and/or psychological, to non-human agents (Duffy, 2003; Epley et al., 2007). Several studies have shown that humans may perceive non-anthropomorphic robots as anthropomorphic, such as Roomba (a vacuum cleaner with a semi-autonomous system; Fink et al., 2012). Although anthropomorphism seems to be a widespread phenomenon, the attribution of human traits to anthropomorphic robots is significantly greater compared to non-anthropomorphic robots. A study by Krach et al. (2008) compared four different agents (computer, functional robot, anthropomorphic robot, and human confederate) in a Prisoner's Dilemma Game, and showed that the more the interactive partner displayed human-like characteristics, the more the participants appreciated the interaction and ascribed intelligence to the game partner. What characteristics of anthropomorphic robots (i.e., the HSRs) increase the perception of anthropomorphism? The HSRs can elicit the perception of anthropomorphism mainly at two levels: physical and behavioral (Marchetti et al., 2018). Working on the physical level is clearly easier than on intrinsic psychological features, and - although anthropomorphic physical features of robots are not the only answer to enhance the quality of interactions with humans - the implementation of these characteristics can positively affect HRIs (Duffy, 2003; for a review see Marchetti et al., 2018). It should be stated, however, that extreme human-likeness can result in the known uncanny valley effect, according to which HRIs are negatively influenced by robots that are too similar to the human (Mori, 1970; MacDorman and Ishiguro, 2006; Mori et al., 2012). Thus, the HSRs' appearance represents an important social affordance for HRIs, as further demonstrated by the psychological research on racial and disability prejudice (Todd et al., 2011; Macdonald et al., 2017; Sarti et al., 2019; Manzi et al., 2020b). The anthropomorphic features of the HSRs can increase humans' perception of humanness, such as mind attribution and personality, and influence other psychological mechanisms and processes (Kiesler and Goetz, 2002; MacDorman et al., 2005; Powers and Kiesler, 2006; Bartneck et al., 2008; Broadbent et al., 2013; Złotowski et al., 2015; Marchetti et al., 2020).

The study of the design of physical characteristics of the HSRs and their classification has been already investigated in HRI, but not systematically. A pioneering study by DiSalvo et al. (2002) explored the perception of humanness using 48 images of different heads of HSRs, and showed that three features are particularly important for the robot's design: the nose, eyes, and mouth. Furthermore, a study by Duffy (2003) categorized different robots' head in a diagram composed of three extremities: "human head" (as-close-as-possible to a human head), "iconic head" (a very minimum set of features) and "abstract head" (a more mechanistic design with minimal human-like aesthetics). Also, in this instance, human likeness was associated with greater mental abilities. Furthermore, a study by MacDorman (2006) analyzed the categorization of 14 types of robots (mainly androids and humanoids) in adults. It was shown that humanoid robots displaying some mechanical characteristics - such as the Robovie robot - were classified average on a "humanness" scale and rated lower on the uncanny valley scale. Recent studies compared one of the most widely used HSRs, the NAO robot, with different types of robots. It was shown that the NAO robot is perceived less human-like than an android - which is a highly anthropomorphic robot in both appearance and behavior (Broadbent, 2017)-, but more anthropomorphic than a mechanical robot, i.e., the Baxter robot (Yogeeswaran et al., 2016; Zanatto et al., 2019). However, there were no differences in perceived ability to perform physical and mental tasks between NAO and the android (Yogeeswaran et al., 2016), indicating that human-likeness (and not "human-exactness") is sufficient to trigger the attribution of psychological features to a robot. 
In addition, a database has recently been created that collects more than 200 HSRs classified according to their level of human likeness (Phillips et al., 2018). In this study the NAO robot was classified with a score of about $45 / 100$, in particular thanks to the characteristics of its face and body. Robovie and other similar robots were classified with a score ranging between 27 and $31 / 100$, deriving mainly from body characteristics. These findings corroborate the hypothesis that NAO and Robovie are two HSRs with different levels of human-likeness due to their physical anthropomorphic features.

The interest in observing the effect of different physical characteristics of robots in terms of attribution of intentions, understanding, and emotions has also been investigated in children (Bumby and Dautenhahn, 1999; Woods et al., 2004; Woods, 2006). In particular, a study by Woods (2006), comparing 40 different robots, revealed that children experience greater discomfort with robots that look too similar to humans, favoring robots with mixed human-mechanical characteristics. These results were confirmed in a recent study by Tung (2016) showing that children preferred robots with not too many human-like features over robots with many human characteristics. Overall, these results suggested that an anthropomorphic design of HSRs may increase children's preference toward them. Still, an excessive implementation of human features can negatively affect the attribution of positive qualities to the robot, again in line with the Uncanny Valley effect above.

\section{Attribution of Mental States}

Different scales were developed to measure psychological anthropomorphism toward robots in adults. These scales typically assess attribution of intelligence, personality and emotions, only to mention a few. In particular, the attribution of internal states to the robot, i.e., to have a mind, is widely used and very promising in HRI (Broadbent et al., 2013; Stafford et al., 2014).

In psychology, the ability to ascribe mental states to others is defined as the Theory of Mind (ToM). Theory of mind is the ability to understand one's own and others' mental states (intentions, emotions, desires, beliefs), and to predict and interpret one's own and others' behaviors on the basis of such meta-representation (Premack and Woodruff, 1978; Wimmer and Perner, 1983; Perner and Wimmer, 1985). Theory of mind abilities develop around four years of age, becoming more sophisticated with development (Wellman et al., 2001). Theory of mind is active not only during humans' relationships but also during interactions with robots (for a review, see Marchetti et al., 2018).

Recent studies have shown that adults tend to ascribe greater mental abilities to robots that have a human appearance (Hackel et al., 2014; Martini et al., 2016). This tendency to attribute human mental states to robots was also observed in children. Generally, children are inclined to anthropomorphize robots by attributing psychological and biological characteristics to them (Katayama et al., 2010; Okanda et al., 2019). Still, they do differentiate between humans and robots' abilities. A pioneering study by Itakura (2008) investigating the attribution of mental verbs to a human and a robot showed that children did not attribute the epistemic verb "think" to the robot. More recent studies have further shown that already from three years of age, children fairly differentiate a human from a robot in terms of mental abilities (Di Dio et al., 2020a), although younger children appear to be more inclined to anthropomorphize robots compared to older children. This effect may be due to the phenomenon of animism, particularly active at three years of age (Di Dio et al., 2020a,b).

\section{Aim of the Study}

The present study aimed to investigate the attribution of mental states (AMS) in children aged 5-9 years to two humanoid robots, $\mathrm{NAO}$ and Robovie, varying in their anthropomorphic physical features (DiSalvo et al., 2002; Duffy, 2003). Differences in the attribution of mental qualities to the two robots were then explored using the robots' degree of physical anthropomorphism and the child's chronological age. The two humanoid robots, NAO and Robovie, have been selected for two main reasons: (1) in relation to their physical appearance, both robots belong to the category of HSRs, but differ for their degree of anthropomorphism (for a detailed description of the robots, see section "Materials"); (2) both robots are largely used in experiments with children (Kanda et al., 2002; Kose and Yorganci, 2011; Kahn et al., 2012; Shamsuddin et al., 2012; Okumura et al., 2013a,b; Tielman et al., 2014; Cangelosi and Schlesinger, 2015, 2018; Hood et al., 2015; Di Dio et al., 2020a,b).

In light of previous findings associated with the use of these specific robots described above, we hypothesized the following: (1) independent of age, children would distinguish between humans and robots in terms of mental states by ascribing lower mental attributes to the robots; (2) children would tend to attribute greater mental qualities to NAO compared to Robovie because of its greater human-likeness; and (3) younger children would tend to attribute more human characteristics to robots (i.e., to anthropomorphize more) than older children.

\section{MATERIALS AND METHODS}

\section{Participants}

Data were acquired on 189 Italian children from kindergarten and primary school age. The children were divided into three age groups for each robot as follows: (1) for the NAO robot, 5 years $(N=24,13$ females; $M=68.14$; $\mathrm{SD}=3.67) ; 7$ years $(N=25,13$ females; $M=91.9 ; \mathrm{SD}=3.43)$; and 9 years $(N=23,12$ females; $M=116.38, \mathrm{SD}=3.91)$; (2) for the Robovie robot, 5 years $(N=33,13$ females; $M=70.9, \mathrm{SD}=2.95) ; 7$ years $(N=49,26$ females; $M=93.4, \mathrm{SD}=3.62)$; and 9 years $(N=35,15$ females; $M=117.42, \mathrm{SD}=4.44)$. The initial inhomogeneity between sample sizes in the NAO and Robovie conditions were corrected by the random selection of children in the Robovie condition, caring to balance by gender. Accordingly, the sample for the Robovie condition used for statistical analysis was composed as follows: 5 years $(N=24,8$ females; $M=70.87, \mathrm{SD}=3.1)$; 7 years $(N=25,14$ females; $M=92.6, \mathrm{SD}=3.73)$; and 9 years $(N=23$, 10 females; $M=117.43, \mathrm{SD}=4.62)$. The children's parents received a written explanation of the procedure of the study, the measurement items, and gave their written consent. The children were not reported by teachers or parents for learning and/or 
socio-relational difficulties. The study was approved by the Local Ethic Committee (Università Cattolica del Sacro Cuore, Milan).

\section{Materials, Task, and Procedure Materials}

The two HSRs selected for this study were the Robovie robot (Hiroshi Ishiguro Laboratories, ATR; Figure 1B) and the NAO robot (Aldebaran Robotics, Figure 1C). We chose these two robots because, although they both belong to the category of HSRs, they differ in their degree of anthropomorphic features (DiSalvo et al., 2002; Duffy, 2003; MacDorman, 2006; Zhang et al., 2008; Phillips et al., 2018). Robovie is a HSR with more abstract anthropomorphic features: no legs but two driving wheels to move, two arms without hands. In particular, the head can be considered "abstract" (Duffy, 2003) because of two important

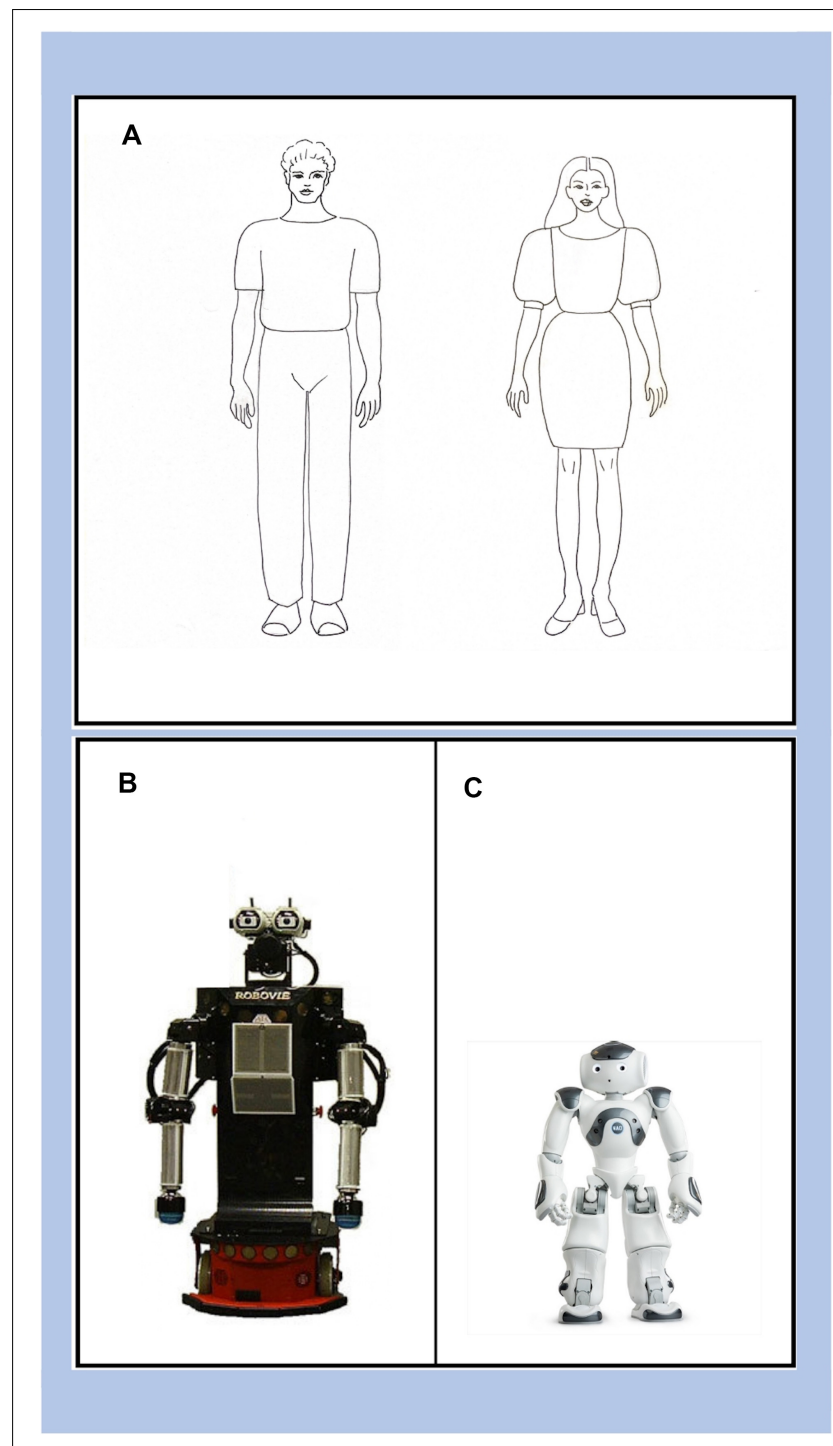

FIGURE 1 | The AMS images: (A) the Human condition (male and female), (B) Robovie robot, and (C) the NAO robot. human-like features: two eyes and a microphone that looks like a mouth (DiSalvo et al., 2002). Robovie is an HSR that can be rated as average in the continuum of mechanical-humanlike (Ishiguro et al., 2001; Kanda et al., 2002; MacDorman, 2006). NAO is a HSR with more pronounced anthropomorphic features compared to Robovie: two legs, two arms, and two hands with three moving fingers (Figure 1C). Besides, the face can be classified as "iconic" and consists of three cameras suggesting two eyes and a mouth. However, considering the whole body and the more detailed shape of the face, NAO is a HSR that can be rated as more human-like than Robovie (DiSalvo et al., 2002; MacDorman, 2006; Phillips et al., 2018).

\section{Attribution of Mental States}

The AMS questionnaire ${ }^{1}$ is a measure of mental states that participants attribute to when they look at images depicting specific characters, in this case a human (female or male based on the participant's gender; Figure 1A), and, according to the group condition, the Robovie or the NAO robot (Figures 1B,C). The AMS questionnaire was inspired by the methodology described in Martini et al. (2016) and is already used in several experiments with children (Manzi et al., 2017; Di Dio et al., 2019, 2020a,b). The construction of the content of the questionnaire is based on the theoretical model of Slaughter et al. (2009) on the categorization of children's mental verbs resulting from communication exchanges between mother and child. This classification divides mental verbs into four categories: perceptive, volitional, cognitive, and dispositional. For the creation of the AMS questionnaire an additional category related to imaginative verbs has been added. We considered it necessary to distinguish between cognitive, epistemic, and imaginative states, since - especially for the robot - this specification enables the analysis of different psychological processes in terms of development. The AMS therefore consists of five dimensions: Perceptive, Emotive, Desires and Intentional, Imaginative, and Epistemic.

The human condition was used as a baseline measure to evaluate children's ability to attribute mental states. In fact, as described in the results below, children scored quite high when ascribing mental attributes to the human character, thus supporting children's competence in performing the mental states attribution task. Also, the human condition was used as a comparison measure against which the level of psychological anthropomorphism of NAO and Robovie was evaluated. The Cronbach's alfa for each category is as follows: Perceptive ( $\alpha=0.8)$, Emotive $(\alpha=0.8)$, Desires and Intentional $(\alpha=0.8)$, Imaginative $(\alpha=0.8)$, and Epistemic ( $\alpha=0.7)$.

Children answered 25 questions grouped into the five different state categories described above (see Appendix 1 for the specific items). The child had to answer "Yes" or "No" to each question, obtaining 1 when the response is "Yes" and 0 when the response is "No". The sum of all responses (range $=0-25$ ) gave the total score $(\alpha=0.9)$; the five partial scores were the sum of the responses within each category (range $=0-5$ ).

${ }^{1}$ http://www.teoriadellamente.it, "Strumenti" section 


\section{Procedure}

The children were tested individually in a quiet room inside their school. Data acquisition was carried out by a single researcher during the normal school activities.

The experimenter showed each child the image on a paper depicting a human - gender matched - and one of the two robots, NAO or Robovie. The presentation order of the image human and robot- was randomized. Afterward, the experimenter asked children the questions on the five categories of the AMS (Perceptive, Emotive, Intentions and Desires, Imaginative, and Epistemic). The presentation order of the five categories was also randomized. The total time required to complete the test was approximately $10 \mathrm{~min}$.

\section{RESULTS}

\section{Data Analysis}

To evaluate the effect of age, gender, states, agent, and type of robots on children's mental state attribution to robots, a GLM analysis was carried out with five levels of states (Perceptive, Emotive, Intentions and Desires, Imaginative, and Epistemic) and two levels of agent (Human, Robot) as within-subjects factors, and age (5-, 7-, 9-year-olds), gender (Male, Female) and robot (Robovie, NAO) as the between-subjects factor. The Greenhouse-Geisser correction was used for violations of Mauchly's Test of Sphericity $(p<0.05)$. Post hoc comparisons were Bonferroni corrected.

\section{Results}

The results showed (1) a main effect of agent, $F(1,126)=570.9$, $p<0.001$, partial $-\eta^{2}=0.819, \delta=1$, indicating that children attributed greater mental states to the human $(M=4.6, \mathrm{SD}=0.27)$ compared to the robot $(M=2.7, \mathrm{SD}=0.21 ; M$ diff $=1.75$, $\mathrm{SE}=0.087)$; (2) a main effect of states, $F(4,504)=40.33, p<0.001$, partial $-\eta^{2}=0.243, \delta=1$, mainly indicating that children attributed greater intention and desires and lower imaginative states (for a full description of the statistics, see Table 1); (3) a main effect of robot, $F(1,126)=39.4, p<0.001$, partial $-\eta^{2}=0.238$, $\delta=1$, showing that children attributed greater mental states to NAO $(M=3.98, \mathrm{SD}=0.17)$ compared to Robovie $(M=3.4$, $\mathrm{SD}=0.14 ; M \operatorname{diff}=0.568, \mathrm{SE}=0.099)$.

A two-way interaction was also found between (1) states and agent, $F(1,126)=16.51, p<0.001$, partial $-\eta^{2}=0.183, \delta=1$ (for a detailed description of the differences see Table 2), and (2) agent and age, $F(2,126)=25.17, p<0.001$, partial $-\eta^{2}=0.285, \delta=1$, showing that 5 -year-old children attributed greater mental states to the robotic agents compared to older children (see Table 2).

Additionally, we found a three-way interaction between states, age, and robot, $F(8,126)=4.95, p<0.001$, partial $-\eta^{2}=0.073$, $\delta=1$. The planned comparisons on the three-way interaction revealed that children attributed greater mental states to NAO compared to Robovie, with the youngest children differentiating on the Perceptive and Epistemic dimensions, and with this difference spreading to all dimensions (but imaginative) in the older children (see Figure 2).
TABLE 1 | Statistics comparing the attribution of all AMS dimensions (Perceptive, Emotive, Intentions and Desires, Imaginative, Epistemic).

\begin{tabular}{|c|c|c|c|c|}
\hline Dimension & Mental States & Mdiff & Err. Stan. & Sign. \\
\hline \multirow[t]{4}{*}{ Perceptive } & Emotive & 0.203 & 0.08 & 0.122 \\
\hline & Int\&Des & $-0.354^{\star}$ & 0.071 & 0.000 \\
\hline & Imaginative & $0.525^{\star}$ & 0.075 & 0.000 \\
\hline & Epistemic & -0.089 & 0.069 & 1 \\
\hline \multirow[t]{4}{*}{ Emotive } & Perceptive & -0.203 & 0.08 & 0.122 \\
\hline & Int\&Des & $-0.557^{\star}$ & 0.074 & 0.000 \\
\hline & Imaginative & $0.322^{*}$ & 0.072 & 0.000 \\
\hline & Epistemic & $-0.292^{\star}$ & 0.079 & 0.004 \\
\hline \multirow[t]{4}{*}{ Int\&Des } & Perceptive & $0.354^{\star}$ & 0.071 & 0.000 \\
\hline & Emotive & $0.557^{\star}$ & 0.074 & 0.000 \\
\hline & Imaginative & $0.879^{*}$ & 0.071 & 0.000 \\
\hline & Epistemic & $0.265^{\star}$ & 0.067 & 0.001 \\
\hline \multirow[t]{4}{*}{ Imaginative } & Perceptive & $-0.525^{\star}$ & 0.065 & 0.000 \\
\hline & Emotive & $-0.322^{*}$ & 0.070 & 0.000 \\
\hline & Int\&Des & $-0.879^{\star}$ & 0.068 & 0.000 \\
\hline & Epistemic & $-0.614^{\star}$ & 0.074 & 0.000 \\
\hline \multirow[t]{4}{*}{ Epistemic } & Perceptive & 0.089 & 0.065 & 1 \\
\hline & Emotive & $0.292^{\star}$ & 0.070 & 0.004 \\
\hline & Int\&Des & $-0.265^{\star}$ & 0.068 & 0.001 \\
\hline & Imaginative & $0.614^{\star}$ & 0.074 & 0.004 \\
\hline
\end{tabular}

Based on estimated marginal averages *The average difference is significant at the level of b Adaptation for multiple comparisons: Bonferroni. Significant values are in bold.

\section{DISCUSSION AND CONCLUSION}

\section{Discussion}

In the present study we compared the AMS in children aged 59 years between two HSRs, NAO and Robovie, also with respect to a human. The aim was to explore children's patterns of mental attribution to different types of HSRs, varying in their degree of physical anthropomorphism, from a developmental perspective.

Our results on the AMS to the human and robot generally confirmed the tendency of children to ascribe lower human mental qualities to the robots, thus supporting previous findings (Manzi et al., 2017; Di Dio et al., 2018, 2019, 2020a,b). In addition, children generally attributed greater mental states to the NAO robot than to the Robovie robot, although differences were found in the quality of mental states attribution as a function of age, with older children discriminating more between the types of robots that the younger ones. As a matter of fact, the important role played by the type of robot in influencing children's AMS can be appreciated by evaluating differences in state attribution developmentally.

Firstly, 5-year-old children generally attributed greater human-like mental states to the robotic agents compared to older children. Additionally, while 5-year-old children discriminated between robots' mental attribution only on the perceptive and epistemic dimensions - with the NAO robot being regarded as more anthropomorphic than Robovie -, children aged 7 and 9 years were particularly sensitive to the type of robots, and attributed greater mental states to NAO than Robovie on most of the tested mental state dimensions. From a 
TABLE 2 | Statistics comparing the attribution of all AMS dimensions (Perceptive, Emotive, Intentions and Desires, Imaginative, Epistemic) and the AMS for the two agents (Human, Robot) across ages (5-, 7- and 9-years).

\begin{tabular}{|c|c|c|c|c|c|c|c|}
\hline & \multirow[b]{2}{*}{ Age } & \multicolumn{3}{|c|}{ Human } & \multicolumn{3}{|c|}{ Robot } \\
\hline & & Mdiff & Err. Stan. & Sign. & Mdiff & Err. Stan. & Sign. \\
\hline & 5 vs 7 & $-0.558^{*}$ & 0.103 & 0.000 & $0.443^{\star}$ & 0.182 & 0.05 \\
\hline & 5 vs 9 & $-0.558^{\star}$ & 0.104 & 0.000 & $0.620^{\star}$ & 0.183 & 0.003 \\
\hline & 7 vs 9 & -0.108 & 0.101 & 0.866 & 0.177 & 0.179 & 0.97 \\
\hline State & Dimensions & Mdiff & Err. Stan. & Sign. & Mdiff & Err. Stan. & Sign. \\
\hline \multirow[t]{4}{*}{ Perceptive } & Emotive & $0.405^{\star}$ & 0.202 & 0.608 & 7,63E-05 & -0.351 & 0.351 \\
\hline & Int\&Des & $0.307^{*}$ & 0.121 & 0.493 & $-0.015^{\star}$ & -1.35 & -0.681 \\
\hline & Imaginative & $0.674^{\star}$ & 0.458 & 0.89 & $0.376^{\star}$ & 0.048 & 0.704 \\
\hline & Epistemic & $0.218^{\star}$ & 0.087 & 0.35 & $-0.396^{\star}$ & -0.758 & -0.035 \\
\hline \multirow[t]{4}{*}{ Emotive } & Perceptive & $-0.405^{\star}$ & -0.608 & -0.202 & -7,63E-05 & -0.351 & 0.351 \\
\hline & Int\&Des & -0.098 & -0.323 & 0.126 & $-1.016^{\star}$ & -1.366 & -0.665 \\
\hline & Imaginative & $0.269^{*}$ & 0.046 & 0.492 & $0.376^{\star}$ & 0.052 & 0.7 \\
\hline & Epistemic & -0.187 & -0.401 & 0.028 & $-0.396^{\star}$ & -0.767 & -0.025 \\
\hline \multirow[t]{4}{*}{ Int\&Des } & Perceptive & $-0.307^{\star}$ & -0.493 & -0.121 & $1.015^{\star}$ & 0.681 & 1.35 \\
\hline & Emotive & 0.098 & -0.126 & 0.323 & $1.016^{\star}$ & 0.665 & 1.366 \\
\hline & Imaginative & $0.367^{*}$ & 0.119 & 0.616 & $1.391^{*}$ & 1.078 & 1.705 \\
\hline & Epistemic & -0.088 & -0.272 & 0.096 & $0.619^{\star}$ & 0.296 & 0.942 \\
\hline \multirow[t]{4}{*}{ Imaginative } & Perceptive & $-0.674^{\star}$ & -0.89 & -0.458 & $-376^{\star}$ & -0.704 & -0.048 \\
\hline & Emotive & $-0.269^{\star}$ & -0.492 & -0.046 & $-0.376^{\star}$ & -0.7 & -0.052 \\
\hline & Int\&Des & $-0.367^{\star}$ & -0.616 & -0.119 & $-1.391^{*}$ & -1.705 & -1.078 \\
\hline & Epistemic & $-0.456^{\star}$ & -0.681 & -0.23 & $-0.772^{\star}$ & -1.112 & -0.432 \\
\hline \multirow[t]{4}{*}{ Epistemic } & Perceptive & $-0.218^{\star}$ & -0.35 & -0.087 & $0.396^{\star}$ & 0.035 & 0.758 \\
\hline & Emotive & 0.187 & -0.028 & 0.401 & $0.396^{*}$ & 0.025 & 0.767 \\
\hline & Int\&Des & 0.088 & -0.096 & 0.272 & $-0.619^{\star}$ & -0.942 & -0.296 \\
\hline & Imaginative & $0.456^{\star}$ & 0.23 & 0.681 & $0.772^{*}$ & 0.432 & 1.112 \\
\hline
\end{tabular}

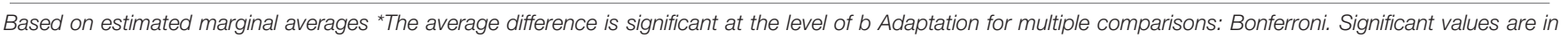
bold.

developmental perspective, the tendency of younger children to anthropomorphize HSRs could be reasonably explained by the phenomenon of animism (Piaget, 1929). Already Piaget in 1929 suggested that children younger than 6 years tend to attribute a consciousness to objects, i.e., the phenomenon of animism, and that this fades around 9 years of age. Recently, this phenomenon has been defined as a cognitive error in children (Okanda et al., 2019), i.e., animism error, characterized by a lack of differentiation between living and non-living things. In this respect, several studies showed that, although children are generally able to discriminate between humans and robots, children aged 5-6 years tend to overuse animistic interpretations for inanimate things, and to attribute biological and psychological properties to robots (Katayama et al., 2010; Di Dio et al., 2019, $2020 a, b)$, in line with the results of this study. Interestingly, we further found a difference in emotional attribution to NAO between 5-year-olds and 7- and 9-year-old children: younger children attributed lower emotions to NAO compared to the older ones. This result may seem counterintuitive in light of what we discussed above; however, by finely looking at the scores obtained from the 5-year-olds for each single emotional question, we found that younger children attributed significantly lower negative emotions to NAO compared to the other age groups, favoring positive emotions $\left(\chi^{2}<0.01\right)$. This resulted in an overall decrease of scores in the emotional dimension for the young children. Therefore, not only does this result not contradict the idea of a greater tendency to anthropomorphize robots in younger children compared to older ones, but also highlights that 5-years-olds perceive NAO as a positive entity that cannot express negative emotions such as anger, sadness, and fear: the "good" play-partner.

From the age of 7 , children's belief of the robots' mind is significantly affected by a sensitivity to the type of the robot, as shown by differences between NAO and Robovie on most mental dimensions, except for Imaginative. The lack of differences between robots on the Imaginative dimension (for all age-groups), which encompasses psychological processes like pretending, and making jokes, appears to be regarded by children as a human prerogative. Interestingly, this result supports findings from a previous study (Di Dio et al., 2018) that compared 6-year-old children's mental state attribution to different entities (human, dog, robot, and God). Also, in that study, imagination was specific to the human entity.

Generally, the findings for older children indicate that the robot's appearance does affect mental state attribution to the robot, and this is increasingly evident with age. However, the judgment of older children could also be significantly influenced by the robot's behavioral characteristics, as demonstrated in 


\section{AMS: states*age* robot}

Perceptive

A

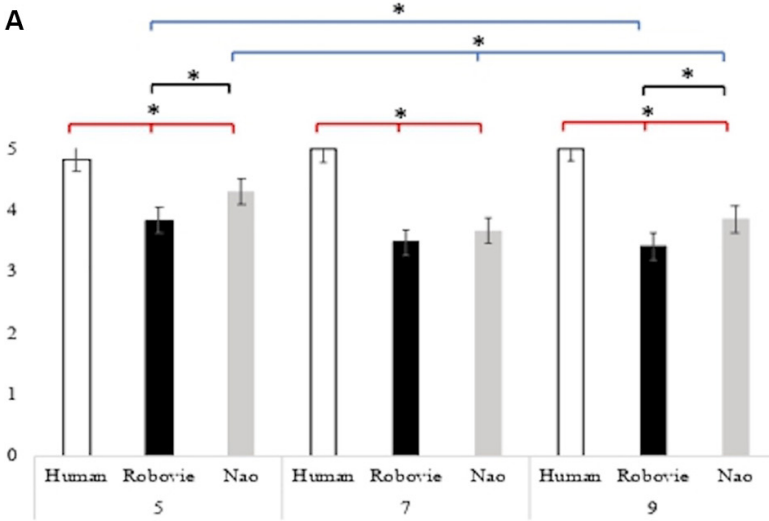

Desires and Intentions

C

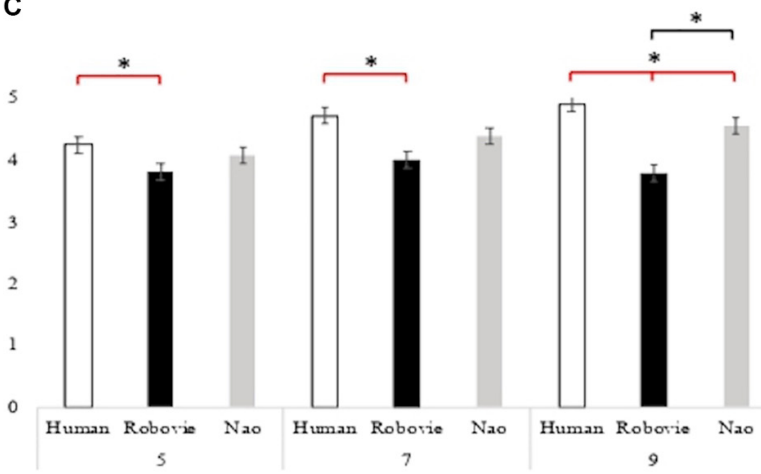

Emotional

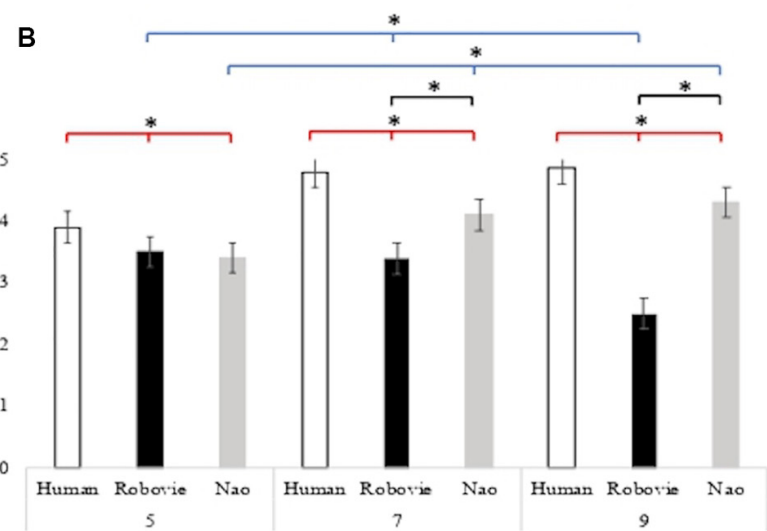

Imaginative

D

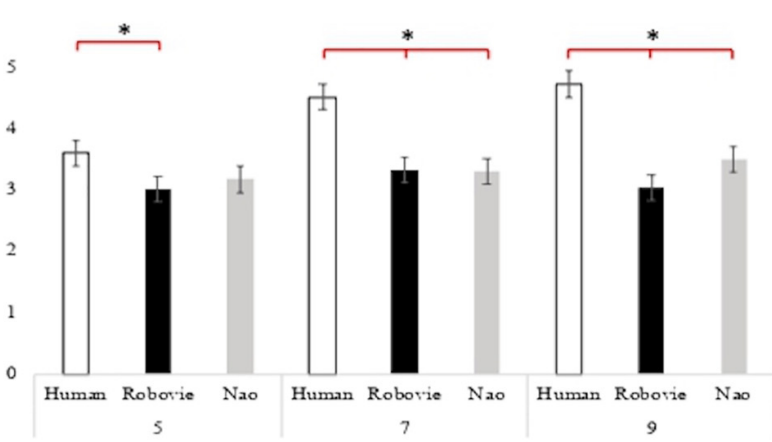

Epistemic

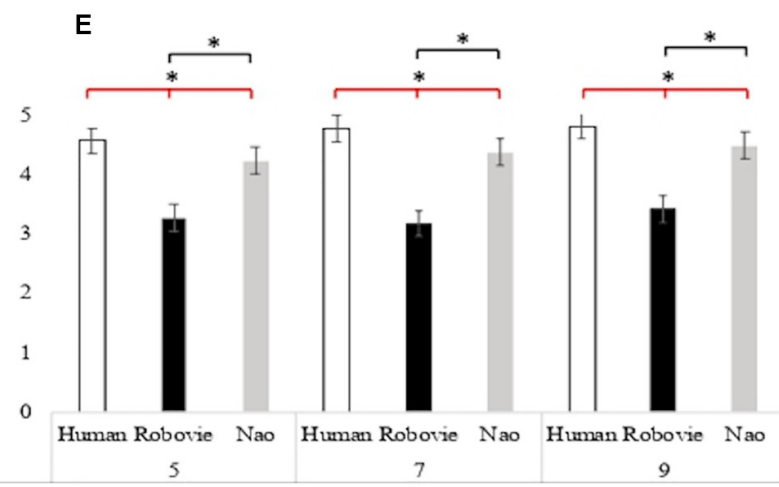

FIGURE 2 | (A-E) Children's scores on the attribution of mental states (AMS) scale. AMS mean scores for the Human (white bar), for Robovie robot (black bar), and NAO robot (gray bar) for each state (Perceptive, Emotions, Intentions and Desires, Imagination, and Epistemic) as a function of age group (5-, 7-, and 9-year-olds). The bars represent the standard error of the mean. ${ }^{*}$ Indicates significant differences. The red lines indicate the differences between agents (Human, Robot); the blue lines indicate the differences between ages (5-, 7-, and 9-year-olds); the black lines indicate the differences between robots (Robovie, NAO).

a long-term study conducted with children aged 10-12 years (Ahmad et al., 2016). In this study, children played a snakes and ladders game with a NAO robot three times across 10 days, whose behavior in terms of personality for a social robot in education was adapted to maintain and create long-term engagement and acceptance. It was found that children positively reacted to the use of the robot in education, stressing a need to implement robots that are able to adapt based on previous experiences in 
real time. Of course, this is very much in line with the great vision of disciplines such DR (Cangelosi and Schlesinger, 2015) and DC (Itakura, 2008). In this respect, it is also important to consider further aspects related to the effectiveness in human relations of constructs such as understanding the perspective of others (e.g., Marchetti et al., 2018) and empathy, on which several research groups are actively working. For example, in an exploratory study Serholt et al. (2014) highlighted the perceived need both for teachers and learners to deal with robots showing such a competence.

In the same vein, other studies that used Robovie as an interactive partner in educational contexts, have also shown that when the robot is programed to facilitate interactional dynamics with children, it can be considered by the children as a group member and even part of the friendship circle. In these studies, the robot is typically programed to act as an effective social communicative partner using strategies, like calling children by their name, or adapting the interactive behaviors for each child by means of behavioral patterns drawn from developmental psychology (Kanda et al., 2007; see also, Kahn et al., 2012). The study by Kahn et al. (2012) further showed that after interacting with Robovie, most children believed that Robovie had mental states (e.g., was intelligent and had feelings) and was a social being (e.g., could be a friend, offer comfort, and be trusted with secrets).

The above studies highlight the prospective use of robots, particularly in the educational field. However, in reality, today's robots are not yet able to sustain autonomous behavior in the long term, even though research is actively laying a good foundation for this. What we can certainly work on with direct effects on children's perception of the mental abilities of robots are their physical attributes. By outlining differences in mental states attribution to different types of humanoid robots across ages based on robots' physical appearance, our findings could help map the design of humanoid robots for children: in early ages, robots can display more abstract and mechanical features (possibly also due to the phenomenon of animism as described above); conversely, in older ages, the tendency to anthropomorphize robots is at least partially affected by the design of the robot. However, it has to be kept in mind that excessive human-likeness may be felt as uncomfortable, as suggested by findings showing that children experience less discomfort with robots displaying both human and mechanical features compared to robots whose physical features markedly evoke human ones (Bumby and Dautenhahn, 1999; Woods et al., 2004; Woods, 2006). Excessive resemblance to the human triggers the Uncanny Valley effect (the more the appearance of robots is similar to humans, the higher the sense of eeriness). These data suggest that a well-designed HSR for children should combine both human and mechanical dimensions, which, in our study, seems to be better represented by the NAO robot.

\section{CONCLUSION}

This study enabled us to analyze the AMS to two types of HSRs, highlighting how different types of robot can evoke different attributions of mental states in children. More specifically, our findings suggest that children's age is an important factor to consider when designing a robot, and provided us with at least two important insights associated with the phenomenon of anthropomorphism from a development perspective, and the design of HSRs for children. Anthropomorphism seems to be a widespread phenomenon in 5-year-olds, while it becomes more dependent on physical features of the robot in older children, with a preference ascribed to the NAO robot that is perceived as more human-like. This effect may then influence the design of robots, which can be more flexible in terms of physical features, as with Robovie, when targeted to young children.

Overall, our results suggest that the assessment of HSRs in terms of mental states attribution may represent a useful measure for studying the effect of different robots' design for children. However, it has to be noted that the current results involved only two types of HSRs. Therefore, future studies will have to evaluate the mental attribution to a greater variety of robots by also comparing anthropomorphic and non-anthropomorphic robots, and across different cultures. In addition, in future studies it will be important to assess children's socio-cognitive abilities such as language, executive functions, and ToM, to analyze the effect of these abilities on the AMS to robots developmentally. Finally, this study explored the mental attributions through images depicting robots. Future studies should include a condition where children interact with the robots in vivo to explore the intersectional effect between the robot's physical appearance and its behavioral patterns. This would enable us to highlight the relative weight of each factor on children's perception of the robots' mental competences.

\section{DATA AVAILABILITY STATEMENT}

The datasets generated for this study are available on request to the corresponding author.

\section{ETHICS STATEMENT}

The studies involving human participants were reviewed and approved by the Ethic Committee, Università Cattolica del Sacro Cuore, Milano, Italy. Written informed consent to participate in this study was provided by the participants' legal guardian/next of kin.

\section{AUTHOR CONTRIBUTIONS}

All the authors conceived and designed the experiment. FM and GP conducted the experiments in schools. AM, FM, and GP secured ethical approval. FM and CDD carried out the statistical analyses. All authors contributed to the writing of the manuscript.

\section{FUNDING}

This publication was granted by Università Cattolica del Sacro Cuore of Milan. 


\section{REFERENCES}

Ahmad, M. I., Mubin, O., and Orlando, J. (2016). "Children views' on social robot's adaptations in education," in Proceedings of the 28th Australian Conference on Computer-Human Interaction (Tasmania: University Of Tasmania), $145-149$.

Airenti, G. (2015). The cognitive bases of anthropomorphism: from relatedness to empathy. Int. J. Soc. Robot. 7, 117-127. doi: 10.1007/s12369-014-0263-x

Bartneck, C., Croft, E., and Kulic, D. (2008). "Measuring the anthropomorphism, animacy, likeability, perceived intelligence and perceived safety of robots," in Proceedings of the Metrics for Human-Robot Interaction Workshop in affiliation with the 3rd ACM/IEEE International Conference on HumanRobot Interaction (HRI 2008), Technical Report 471 (Piscataway, NJ: IEEE), $37-44$.

Bartneck, C., and Forlizzi, J. (2004). "A design-centered framework for social human-robot interaction," in Preceedings of the RO-MAN 2004. 13th IEEE International Workshop on Robot and Human Interactive Communication (Piscataway, NJ: IEEE), 591-594.

Bartneck, C., Kanda, T., Mubin, O., and Al Mahmud, A. (2009). Does the design of a robot influence its animacy and perceived intelligence? Int. J. Soc. Robot. 1, 195-204. doi: 10.1007/s12369-009-0013-7

Begum, M., Serna, R. W., and Yanco, H. A. (2016). Are robots ready to deliver autism interventions? A comprehensive review. Int. J. Soc. Robot. 8, 157-181. doi: 10.1007/s12369-016-0346-y

Belpaeme, T., Kennedy, J., Ramachandran, A., Scassellati, B., and Tanaka, F. (2018). Social robots for education: a review. Sci. Robot. 3:eaat5954. doi: 10.1126/ scirobotics.aat5954 doi: 10.1016/j.ijhcs.2011.04.003.

Broadbent, E. (2017). Interactions with robots: the truths we reveal about ourselves. Annu. Rev. Psychol. 68, 627-652. doi: 10.1146/annurev-psych-010416-043958

Broadbent, E., Kumar, V., Li, X., Sollers, J., Stafford, R. Q., MacDonald, B. A., et al. (2013). Robots with display screens: A robot with a more humanlike face display is perceived to have more mind and a better personality. PLoS One 8:e72589. doi: 10.1371 /journal.pone.0072589

Bumby, K., and Dautenhahn, K. (1999). “Investigating children's attitudes towards robots: A case study," in Proceedings of the CT99, The 3rd International Cognitive Technology Conference, San Francisco, 391-410.

Cangelosi, A., and Schlesinger, M. (2015). Developmental Robotics: from Babies to Robots. London: MIT Press.

Cangelosi, A., and Schlesinger, M. (2018). From babies to robots: the contribution of developmental robotics to developmental psychology. Child Dev. Perspect. 12, 183-188. doi: 10.1111/cdep.12282

Dario, P., Guglielmelli, E., and Laschi, C. (2001). Humanoids and personal robots: design and experiments. J. Robot. Syst. 18, 673-690. doi: 10.1002/rob. 8106

Dautenhahn, K., Nehaniv, C. L., Walters, M. L., Robins, B., Kose-Bagci, H., Mirza, N. A., et al. (2009). KASPAR-a minimally expressive humanoid robot for human-robot interaction research. Appl. Bionics Biomech. 6, 369-397. doi: $10.1155 / 2009 / 708594$

De La Cruz, V. M., Di Nuovo, A., Di Nuovo, S., and Cangelosi, A. (2014). Making fingers and words count in a cognitive robot. Front. Behav. Neurosci. 8:13. doi: $10.3389 /$ fnbeh.2014.00013

Di Dio, C., Isernia, S., Ceolaro, C., Marchetti, A., and Massaro, D. (2018). Growing up Thinking of God's Beliefs: Theory of Mind and Ontological Knowledge. Thousand Oaks, CA: Sage Open, 1-14. doi: 10.1177/2158244018809874

Di Dio, C., Manzi, F., Itakura, S., Kanda, T., Hishiguro, H., Massaro, D., et al. (2019). It does not matter who you are: fairness in pre-schoolers interacting with human and robotic partners. Int. J. Soc. Robot. 1-15. doi: 10.1007/s12369019-00528-9

Di Dio, C., Manzi, F., Peretti, G., Cangelosi, A., Harris, P. L., Massaro, D., et al. (2020a). Come i bambini pensano alla mente del robot. Il ruolo dell'attaccamento e della Teoria della Mente nell'attribuzione di stati mentali ad un agente robotico. Sistemi Intell. 32, 41-56. doi: 10.1422/96279

Di Dio, C., Manzi, F., Peretti, G., Cangelosi, A., Harris, P. L., Massaro, D., et al. (2020b). Shall I trust you? From child human-robot interaction to trusting relationships. Front. Psychol. 11:469. doi: 10.3389/fpsyg.2020.00469

DiSalvo, C. F., Gemperle, F., Forlizzi, J., and Kiesler, S. (2002). "All robots are not created equal: The design and perception of humanoid robot heads,"
Proceedings of the 4th conference on Designing Interactive Systems: Processes, Practices, Methods, and Techniques. (New York, NY: ACM).

Duffy, B. R. (2003). Anthropomorphism and the social robot. Robot. Auton. Syst. 42, 177-190. doi: 10.1016/s0921-8890(02)00374-3

Epley, N., Waytz, A., and Cacioppo, J. T. (2007). On seeing human: a three-factor theory of anthropomorphism. Psychol. Rev. 114, 864-886. doi: 10.1037/0033295x.114.4.864

Fink, J., Mubin, O., Kaplan, F., and Dillenbourg, P. (2012). “Anthropomorphic language in online forums about Roomba, AIBO and the iPad," in Proceedings of the 2012 IEEE Workshop on Advanced Robotics and its Social Impacts (ARSO), Munich: IEEE, 54-59.

Hackel, L. M., Looser, C. E., and Van Bavel, J. J. (2014). Group membership alters the threshold for mind perception: the role of social identity collective identification and intergroup threat. J. Exp. Soc. Psychol. 2014, 15-23. doi: 10.1016/j.jesp.2013.12.001

Hood, D., Lemaignan, S., and Dillenbourg, P. (2015). "When children teach a robot to write: An autonomous teachable humanoid which uses simulated handwriting," in Proceedings of the Tenth Annual ACM/IEEE International Conference on Human-Robot Interaction (Piscataway, NJ: IEEE) 83-90.

Ishiguro, H., Ono, T., Imai, M., Kanda, T., and Nakatsu, R. (2001). Robovie: an interactive humanoid robot. Int. J. Industrial Robot 28, 498-503. doi: 10.1108/ 01439910110410051

Itakura, S. (2008). Development of mentalizing and communication: from viewpoint of developmental cybernetics and developmental cognitive neuroscience. IEICE Trans. Commun. 2109-2117. doi: 10.1093/ietcom/ e91-b.7.2109

Itakura, S., Ishida, H., Kanda, T., Shimada, Y., Ishiguro, H., and Lee, K. (2008). How to build an intentional android: infants' imitation of a robot's goal-directed actions. Infancy 13, 519-532. doi: 10.1080/15250000802329503

Kahn, P. H. Jr., Kanda, T., Ishiguro, H., Freier, N. G., Severson, R. L., Gill, B. T., et al. (2012). "Robovie, you'll have to go into the closet now": children's social and moral relationships with a humanoid robot. Dev. Psychol. 48:303. doi: $10.1037 / \mathrm{a} 0027033$

Kanda, T., Ishiguro, H., Ono, T., Imai, M., and Mase, K. (2002). "Development and Evaluation of an Interactive Robot "Robovie", in Proceedings of the IEEE International Conference on Robotics and Automation, Washington, DC, 18481855. doi: 10.1109/ROBOT.2002.1014810

Kanda, T., Sato, R., Saiwaki, N., and Ishiguro, H. (2007). A two-month field trial in an elementary school for long-term human-robot interaction. IEEE Trans. Robot. 23, 962-971. doi: 10.1109/TRO.2007.904904

Kannegiesser, P., Itakura, S., Zhou, Y., Kanda, T., Ishiguro, H., and Hood, B. (2015). The role of social eye-gaze in children's and adult's ownership attributions to robotic agents in three cultures. Interact. Stud. 16, 1-28. doi: 10.1075/is.16.1. $01 \mathrm{kan}$

Katayama, N., Katayama, J. I., Kitazaki, M., and Itakura, S. (2010). Young children's folk knowledge of robots. Asian Cult. Hist. 2:111.

Kiesler, S., and Goetz, J. (2002). "Mental models of robotic assistants," in Proceedings of the CHI'02 Extended Abstracts on Human Factors in Computing Systems (New York, NY: ACM), 576-577.

Kiesler, S., Powers, A., Fussell, S. R., and Torrey, C. (2008). Anthropomorphic interactions with a robot and robot-like agent. Soc. Cogn. 26, 169-181. doi: 10.1521/soco.2008.26.2.169

Kose, H., and Yorganci, R. (2011). "Tale of a robot: Humanoid robot assisted sign language tutoring," in Proceedings of the IEEE International Conference on Robotics and Automation2011 11th IEEE-RAS International Conference on Humanoid Robots (Piscataway, NJ: IEEE), 105-111.

Krach, S., Hegel, F., Wrede, B., Sagerer, G., Binkofski, F., and Kircher, T. (2008). Can machines think? Interaction and perspective taking with robots investigated via fMRI. PLoS One 3:e2597. doi: 10.1371/journal.pone.0002597

Lyon, C., Nehaniv, L. C., Saunders, J., Belpaeme, T., Bisio, A., Fischer, K., and Cangelosi, A. (2016). Embodied language learning and cognitive bootstrapping: methods and design principles. Int. J. Adv. Robotic Syst. 13:105. doi: 10.5772/ 63462

Macdonald, S. J., Donovan, C., and Clayton, J. (2017). The disability bias: understanding the context of hate in comparison with other minority populations. Disabil. Soc. 32, 483-499. doi: 10.1080/09687599.2017.1304206

MacDorman, K. F. (2006). "Subjective ratings of robot video clips for human likeness, familiarity, and eeriness: An exploration of the uncanny valley," 
in Proceedings of the ICCS/CogSci-2006 Long Symposium: Toward social Mechanisms of Android Science, Vancouver, 26-29.

MacDorman, K. F., and Ishiguro, H. (2006). The uncanny advantage of using androids in cognitive and social science research. Interact. Stud. 7, 297-337. doi: $10.1075 /$ is.7.3.03mac

MacDorman, K. F., Minato, T., Shimada, M., Itakura, S., Cowley, S., and Ishiguro, H. (2005). "Assessing human likeness by eye contact in an android testbed," in Proceedings of the 27th annual meeting of the cognitive science society (Austin, TX: CSS) 21-23.

Manzi, F., Ishikawa, M., Di Dio, C., Itakura, S., Kanda, T., Ishiguro, H., et al. (2020a). The understanding of congruent and incongruent referential gaze in 17-month-old infants: An eye-tracking study comparing human and robot. Sci. Rep. 10:11918. doi: 10.1038/s41598-020-69140-6

Manzi, F., Massaro, D., Kanda, T., Tomita, K., Itakura, S., and Marchetti, A. (2017). “Teoria della Mente, bambini e robot: l'attribuzione di stati mentali," in Proceedings of the Abstract de XXX Congresso Nazionale, Associazione Italiana di Psicologia, Sezione di Psicologia dello Sviluppo e dell'Educazione (Messina, 14-16 September 2017), Italy: Alpes Italia srl, 65-66. Available online at: http: //hdl.handle.net/10807/106022

Manzi, F., Savarese, G., Mollo, M., and Iannaccone, A. (2020b). Objects as communicative mediators in children with autism spectrum disorder. Front. Psychol. 11:1269. doi: 10.3389/fpsyg.2020.01269

Marchetti, A., Di Dio, C., Manzi, F., and Massaro, D. (in press). "Robotics in clinical and developmental psychology," in Comprehensive Clinical Psychology, 2nd Edn, Amsterdam: Elsevier.

Marchetti, A., Di Dio, C., Manzi, F., and Massaro, D. (2020). The psychosocial fuzziness of fear in the COVID-19 era and the role of robots. Front. Psychol. 10:2245. doi: 10.3389/fpsyg.2020.02245

Marchetti, A., Manzi, F., Itakura, S., and Massaro, D. (2018). Theory of Mind and humanoid robots from a lifespan perspective. Z. Psychol. 226, 98-109. doi: 10.1027/2151-2604/a000326

Martini, M. C., Gonzalez, C. A., and Wiese, E. (2016). Seeing minds in others Can agents with robotic appearance have human-like preferences? PLoS One 11:e0146310. doi: 10.1371/journal.pone. 0146310

Mori, M. (1970). The uncanny valley. Energy 7, 33-35

Mori, M., MacDorman, K. F., and Kageki, N. (2012). The uncanny valley [from the field]. IEEE Robot. Autom. Mag. 19, 98-100. doi: 10.1109/mra.2012.219 2811

Moriguchi, Y., Kanda, T., Ishiguro, H., Shimada, Y., and Itakura, S. (2011). Can young children learn words from a robot? Interact. Stud. 12, 107-119. doi: $10.1075 /$ is. 12.1 .04 mor

Morse, A. F., and Cangelosi, A. (2017). Why are there developmental stages in language learning? A developmental robotics model of language development. Cognitive Sci. 41, 32-51. doi: 10.1111/cogs.12390

Mubin, O., Stevens, C. J., Shahid, S., Al Mahmud, A., and Dong, J. J. (2013). A review of the applicability of robots in education. J. Technol. Educ. Learn. 1:13.

Murashov, V., Hearl, F., and Howard, J. (2016). Working safely with robot workers: Recommendations for the new workplace. J. Occup. Environ. Hyg. 13, D61-D71.

Okanda, M., Taniguchi, K., and Itakura, S. (2019). "The role of animism tendencies and empathy in adult evaluations of robot," in Proceedings of the 7th International Conference on Human-Agent Interaction, HAI '19 (New York, NY: Association for Computing Machinery), 51-58. doi: 10.1145/3349537.3351891

Okanda, M., Zhou, Y., Kanda, T., Ishiguro, H., and Itakura, S. (2018). I hear your yes -no questions: Children's response tendencies to a humanoid robot. Infant Child Dev. 27:e2079. doi: 10.1002/icd.2079

Okumura, Y., Kanakogi, Y., Kanda, T., Ishiguro, H., and Itakura, S. (2013a). Can infants use robot gaze for object learning? The effect of verbalization. Int. Stud. 14, 351-365. doi: 10.1075/is.14.3.03oku

Okumura, Y., Kanakogi, Y., Kanda, T., Ishiguro, H., and Itakura, S. (2013b). Infants understand the referential nature of human gaze but not robot gaze. J. Exp. Child Psychol. 116, 86-95. doi: 10.1016/j.jecp.2013.02.007

Perner, J., and Wimmer, H. (1985). "John thinks that Mary thinks that." attribution of second-order beliefs by 5- to 10-year- old children. J. Exp. Child Psychol. 39, 437-471. doi: 10.1016/0022-0965(85)90051-7

Phillips, E., Zhao, X., Ullman, D., and Malle, B. F. (2018). "What is human-like? Decomposing robots' human-like appearance using the anthropomorphic roBOT (ABOT) database," in Proceedings of the 2018
ACM/IEEE International Conference on Human-Robot Interaction. (Piscataway, NJ: IEEE).

Piaget, J. (1929). The Child's Conception of the World. London: Routledge.

Powers, A., and Kiesler, S. (2006). "The advisor robot: tracing people's mental model from a robot's physical attributes," in Proceedings of the 1st ACM SIGCHI/SIGART Conference on Human-Robot Interaction (New York, NY: ACM), 218-225.

Premack, D., and Woodruff, G. (1978). Does the chimpanzee have a theory of mind. Behav. Brain Sci. 1, 515-526. doi: 10.1017/s0140525x000 76512

Sarti, D., Bettoni, R., Offredi, I., Tironi, M., Lombardi, E., Traficante, D., et al. (2019). Tell me a story: socio-emotional functioning, well-being and problematic smartphone use in adolescents with specific learning disabilities. Front. Psychol. 10:2369. doi: 10.3389/fpsyg.2019.02369

Serholt, S., Barendregt, W., Leite, I., Hastie, H., Jones, A., Paiva, A., et al. (2014). 'Teachers' views on the use of empathic robotic tutors in the classroom," in Preceedings of the 23rd IEEE International Symposium on Robot and Human Interactive Communication (Piscataway, NJ: IEEE), 955-960.

Shamsuddin, S., Yussof, H., Ismail, L. I., Mohamed, S., Hanapiah, F. A., and Zahari, N. I. (2012). Initial response in HRI-a case study on evaluation of child with autism spectrum disorders interacting with a humanoid robot Nao. Procedia Eng. 41, 1448-1455. doi: 10.1016/j.proeng.2012. 07.334

Sharkey, A., and Sharkey, N. (2011). Children, the elderly, and interactive robots. IEEE Robot. Autom. Mag. 18, 32-38. doi: 10.1109/mra.2010.94 0151

Shiomi, M., Kanda, T., Ishiguro, H., and Hagita, N. (2006). "Interactive humanoid robots for a science museum," in Proceedings of the 1st ACM SIGCHI/SIGART Conference on Human-robot Interaction (New York, NY: ACM) 305-312.

Slaughter, V., Peterson, C. C., and Carpenter, M. (2009). Maternal mental state talk and infants' earlygestural communication. J. Child Lang. 36, 1053-1074. doi: 10.1017/S0305000908009306

Stafford, R. Q., MacDonald, B. A., Jayawardena, C., Wegner, D. M., and Broadbent, E. (2014). Does the robot have a mind? Mind perception and attitudes towards robots predict use of an eldercare robot. Int. J. Soc. Robot. 6, 17-32. doi: 10.1007/s12369-013-0186-y

Tielman, M., Neerincx, M., Meyer, J. J., and Looije, R. (2014). “Adaptive emotional expression in robot-child interaction," in Proceedings of the 2014 9th ACM/IEEE International Conference on Human-Robot Interaction (HRI) (Piscataway, NJ: IEEE), 407-414.

Todd, A. R., Bodenhausen, G. V., Richeson, J. A., and Galinsky, A. D. (2011). Perspective taking combats automatic expressions of racial bias. J. Pers. Soc. Psychol. 100, 1027-1042. doi: 10.1037/a0022308

Tung, F. W. (2016). Child perception of humanoid robot appearance and behavior. Int. J. Hum. Comput. Interact. 32, 493-502. doi: 10.1080/10447318.2016. 1172808

Vinanzi, S., Patacchiola, M., Chella, A., and Cangelosi, A. (2019). Would a robot trust you? Developmental robotics model of trust and theory of mind. Philos. Tr. R. Soc. B 374:20180032. doi: 10.1098/rstb.2018.0032

Wainer, J., Robins, B., Amirabdollahian, F., and Dautenhahn, K. (2014). Using the humanoid robot KASPAR to autonomously play triadic games and facilitate collaborative play among children with autism. IEEE Trans. Auton. Ment. Dev. 6, 183-199. doi: 10.1109/tamd.2014.2303116

Wang, Y., Park, Y. H., Itakura, S., Henderson, A. M. E., Kanda, T., Furuhata, N., et al. (2020). Infants' perceptions of cooperation between a human and robot. Infant Child Dev. 29:e2161. doi: 10.1002/icd.2161

Wellman, H. M., Cross, D., and Watson, J. (2001). Meta-analysis of theory-ofmind development: the truth about false belief. Child Dev. 72, 655-684. doi: $10.1111 / 1467-8624.00304$

Wimmer, H., and Perner, J. (1983). Beliefs about beliefs: Representation and constraining function of wrong beliefs in young children's understanding of deception. Cognition 13, 103-128. doi: 10.1016/0010-0277(83)90 004-5

Woods, S. (2006). Exploring the design space of robots: Children's perspectives. Interact. Comput. 18, 1390-1418. doi: 10.1016/j.intcom.2006. 05.001 
Woods, S., Dautenhahn, K., and Schulz, J. (2004). "The design space of robots: Investigating children's views," in Proceedings of the 13th IEEE International Workshop on Robot and Human Interactive Communication (Piscataway, NJ: IEEE), 47-52.

Yogeeswaran, K., Złotowski, J., Livingstone, M., Bartneck, C., Sumioka, H., and Ishiguro, H. (2016). The interactive effects of robot anthropomorphism and robot ability on perceived threat and support for robotics research. J. Hum. Robot Interact. 5, 29-47. doi: 10.5898/jhri.5.2.yogeeswaran

Zanatto, D., Patacchiola, M., Cangelosi, A., and Goslin, J. (2020). Generalisation of Anthropomorphic stereotype. Int. J. Soc. Robot. 12, 163-172. doi: 10.1007/ s12369-019-00549-4

Zanatto, D., Patacchiola, M., Goslin, J., and Cangelosi, A. (2016). "Priming anthropomorphism: can the credibility of humanlike robots be transferred to non-humanlike robots?" in Proceedings of the 2016 11th ACM/IEEE International Conference on Human-Robot Interaction (HRI), Christchurch: IEEE, 543-544. doi: 10.1109/HRI.2016.7451847

Zanatto, D., Patacchiola, M., Goslin, J., and Cangelosi, A. (2019). Investigating cooperation with robotic peers. PLoS One 14:e0225028. doi: 10.1371/journal. pone. 0225028

Zhang, T., Zhu, B., Lee, L., and Kaber, D. (2008). “Service robot anthropomorphism and interface design for emotion in human-robot interaction," in Proceedings of the 2008 IEEE International Conference on Automation Science and Engineering (Piscataway, NJ: IEEE), 674-679.

Zhong, J., Ogata, T., Cangelosi, A., and Yang, C. (2019). Disentanglement in conceptual space during sensorimotor interaction. Cogn. Comput. Syst. 1, 103-112. doi: 10.1049/ccs.2019.0007

Złotowski, J., Proudfoot, D., Yogeeswaran, K., and Bartneck, C. (2015). Anthropomorphism: opportunities and challenges in human-robot interaction. Int. J Soc. Robot. 7, 347-360. doi: 10.1007/s12369-0140267-6

Conflict of Interest: The authors declare that the research was conducted in the absence of any commercial or financial relationships that could be construed as a potential conflict of interest.

Copyright (c) 2020 Manzi, Peretti, Di Dio, Cangelosi, Itakura, Kanda, Ishiguro, Massaro and Marchetti. This is an open-access article distributed under the terms of the Creative Commons Attribution License (CC BY). The use, distribution or reproduction in other forums is permitted, provided the original author(s) and the copyright owner(s) are credited and that the original publication in this journal is cited, in accordance with accepted academic practice. No use, distribution or reproduction is permitted which does not comply with these terms. 


\section{APPENDIX 1}

\section{Attribution of Mental States (AMS)}

I will show you an image of a girl/boy/robot (to be selected depending on condition). I will ask you some questions about her/him/it (depending on condition). You can answer Yes or No to the questions.

Dimensions (5) and questions (25)

\section{Perceptive}

Do you think she/he/it can smell?

Do you think she/he/it can see?

Do you think she/he/it can taste?

Do you think she/he/it can hear?

Do you think she/he/it can feel hot or cold?

\section{Emotive}

Do you think she/he/it can get angry?

Do you think she/he/it can be scared?

Do you think she/he/it can be happy?

Do you think she/he/it can be surprised?

Do you think she/he/it can be sad?

\section{Intentions and desires}

Do you think she/he/it may have the intention to do something?

Do you think she/he/it might want to do something?

Do you think she/he/it might be willing to do something?

Do you think she/he/it can make a wish?

Do you think she/he/it might prefer one thing over another?

\section{Imaginative}

Do you think she/he/it can tell a lie?

Do you think she/he/it can pretend?

Do you think she/he/it can imagine?

Do you think she/he/it can make a joke?

Do you think she/he/it can dream?

\section{Epistemic}

Do you think she/he/it can understand?

Do you think she/he/it can make a decision?

Do you think she/he/it can learn?

Do you think she/he/it can teach?

Do you think she/he/it can think?

http://www.teoriadellamente.it, "Strumenti" section. 


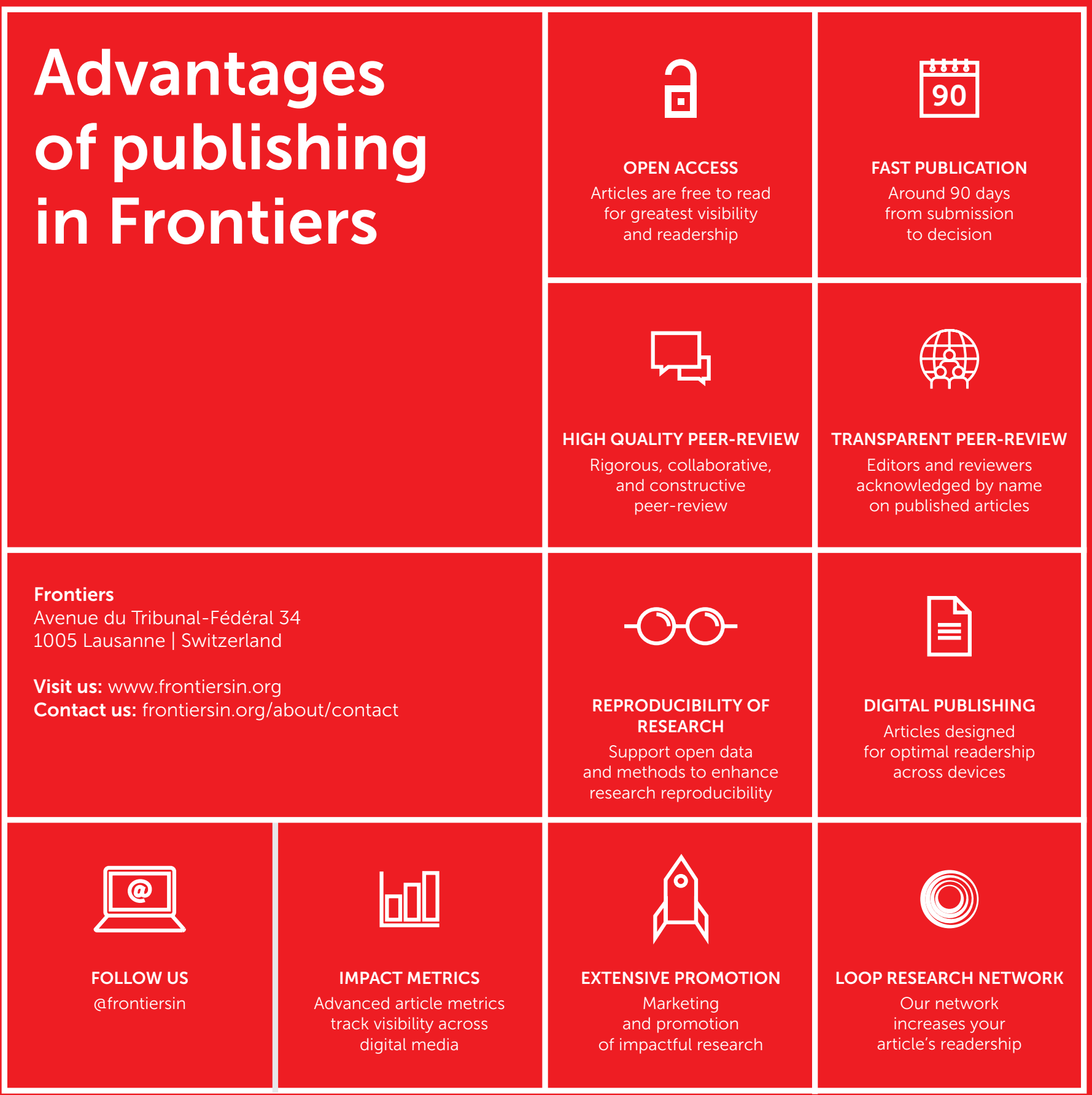

